\title{
Die ökologische Bedeutung von Hecken für Vögel
}

I

Das Heckenprogramm der deutschen Vogelwarten - Netzfang und Revierkartierung zur Erfassung populationsdynamischer und reproduktionsbiologischer Aspekte in einem anthropogen geformten Lebensraum

\author{
II \\ Populationsbiologische Bedeutung von Hecken \\ für Vögel in der Kulturlandschaft
}

Dissertation zur Erlangung des Doktorgrades der MathematischNaturwissenschaftlichen Fakultäten der Georg-August-Universität zu Göttingen

Angefertigt am Institut für Vogelforschung 'Vogelwarte Helgoland' in Wilhelmshaven und am Zentrum für Naturschutz der Georg-August-Universität Göttingen

vorgelegt von:

\author{
Andreas Barkow \\ aus Bremen
}

Göttingen 2001 


\section{Die ökologische Bedeutung von Hecken für Vögel}

Die vorliegende Arbeit zur ökologischen Bedeutung von Hecken für Vögel gliedert sich in zwei inhaltlich verbundene, aber unabhängig voneinander durchgeführte Studien.

I Das Heckenprogramm der deutschen Vogelwarten - Netzfang und Revierkartierung zur Erfassung populationsdynamischer und reproduktionsbiologischer Aspekte in einem anthropogen geformten Lebensraum

Das 'Heckenprogramm' basiert auf Erhebungen ehrenamtlicher Mitarbeiter der Vogelwarten, die über einen Zeitraum von fünf Jahren unter standardisierten Bedingungen Vögel in Hecken mit Netzen gefangen und individuell markiert und zudem Brutbestandskartierungen durchgeführt haben. Mein Beitrag zu dieser Studie war die Verwaltung und Auswertung der Daten und die Übernahme der Betreuung der Beringer nach Anlauf des Programms.

\section{Populationsbiologische Bedeutung von Hecken für Vögel in der Kulturlandschaft}

Die Freilandstudie zur Populationsökologie von Heckenvögeln wurde von mir zwischen 1998 und 2000 in der Kulturlandschaft um Göttingen durchgeführt. Inhaltlich knüpft die Fragestellung an das Heckenprogramm an und behandelt darin aufgeworfene Fragen, z.B. zum Bruterfolg einzelner Arten oder die besonderen Prädationsverhältnisse in einem linearen Lebensraum.

Die Arbeit wurde mit einem Stipendium der Deutsche Bundesstiftung Umwelt gefördert.

Die Durchführung der Arbeit erfolgte am Institut für Vogelforschung 'Vogelwarte Helgoland' in Wilhelmshaven und am Zentrum für Naturschutz der Universität Göttingen.

Betreuer der Arbeit: $\quad$ Prof. Dr. F. Bairlein

Referent:

Prof. Dr. M. Mühlenberg

Koreferent:

Prof. Dr. M. Schaefer

Tag der mündlichen Prüfung: 31. Oktober 2001 
Inhalt

I Das Heckenprogramm der deutschen Vogelwarten - Netzfang und Revierkartierung zur Erfassung populationsdynamischer und reproduktionsbiologischer Aspekte in einem anthropogen geformten Lebensraum

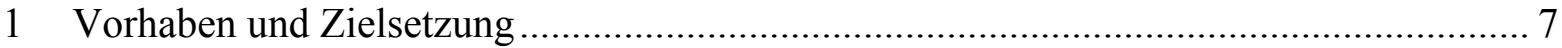

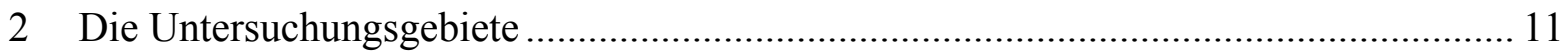

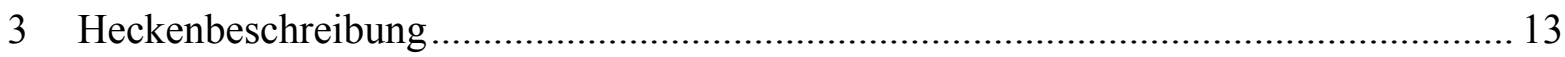

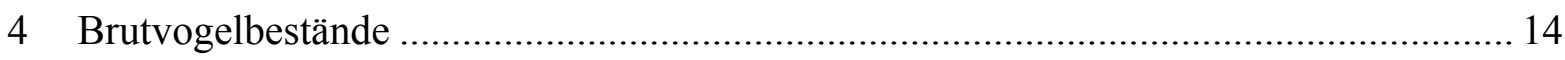

4.1 Methode: Revierkartierung und Linientaxierung............................................. 14

4.2 Arten und Abundanzen des Brutvogelbestandes................................................... 14

4.3 Besiedlungsdichte und Diversität von Brutvögeln in Hecken ................................. 19

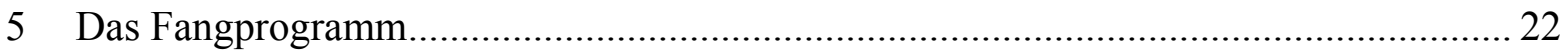

5.1 Methode: Standardisierter Netzfang durch konstanten Aufwand in der Brutzeit..... 22

5.1.1 Durchführung und Durchführbarkeit des standardisierten Netzfangs............... 23

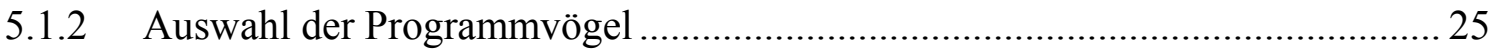

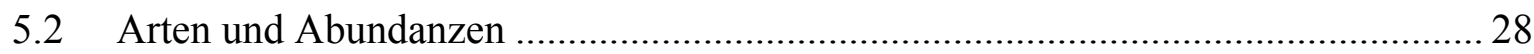

5.2.1 Gibt es bevorzugte Heckenstrukturen?................................................... 31

5.2.1.1 Dominanz einzelner Arten in unterschiedlichen Heckentypen...................... 33

5.2.1.2 Dominanz von Vogelarten in Hecken mit Waldcharakter und Hecken mit weniger ausgeprägten Gehölzstrukturen ................................................ 38

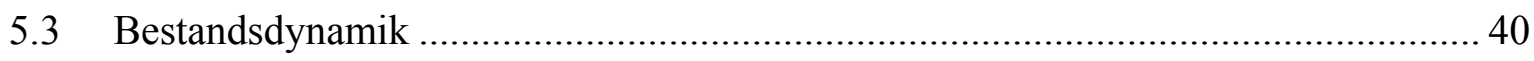

5.3.1 Fangsummen und Bestandsveränderungen .................................................. 41

5.3.2 Abundanzveränderungen einzelner Arten in Relation zum Gesamtfang ........... 43

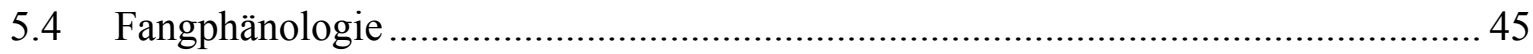

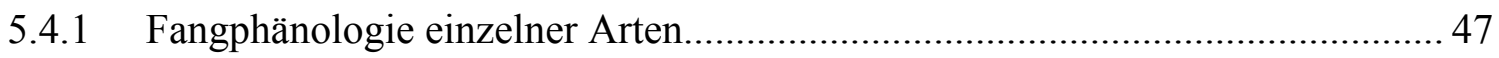

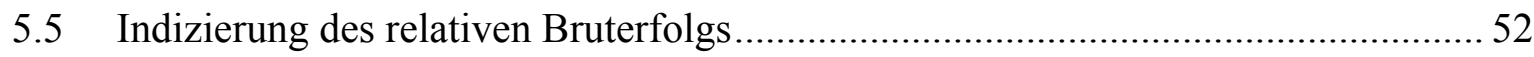

5.5.1 Festlegung der Zeitintervalle zur Berechnung von Bruterfolgs-Indizes ............ 52

5.5.2 Der relative Bruterfolg häufiger Brutvögel in Hecken.....................................5 57 


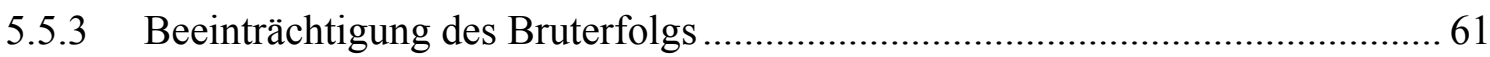

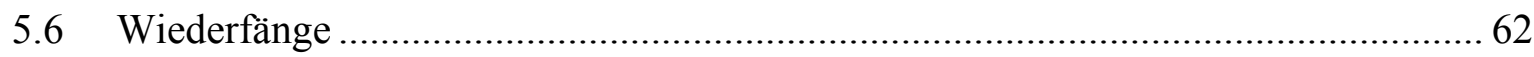

5.6.1 Entwicklung der Wiederfangraten über die Jahre ........................................ 63

5.6.2 Wann treffen die Revierinhaber in den Brutgebieten ein? .............................. 65

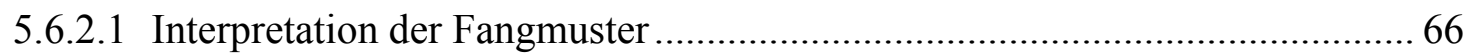

5.6.3 Wieviel Zeit liegt zwischen dem Erstfang und dem Wiederfang? ..................... 67

6 Vergleich der Fangergebnisse mit den Daten der Revierkartierungen .......................... 69

6.1 Korrelation der absoluten Anzahl von Arten zwischen Fang und Kartierung .......... 69

6.1.1 Vergleich der Häufigkeitsverhältnisse der Arten zueinander (Dominanz) ........ 70

6.2 Wie gut lässt sich der Brutvogelbestand anhand von Wiederfängen abschätzen? ... 71

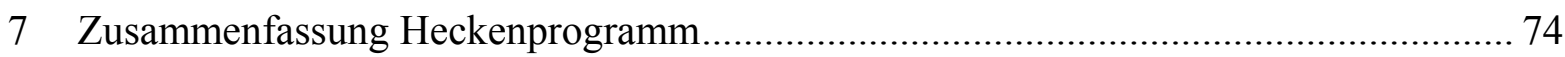

II Populationsbiologische Bedeutung von Hecken für Vögel in der Kulturlandschaft

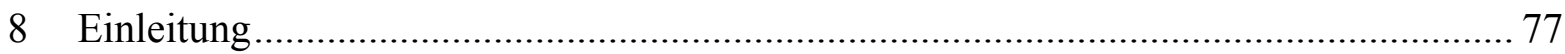

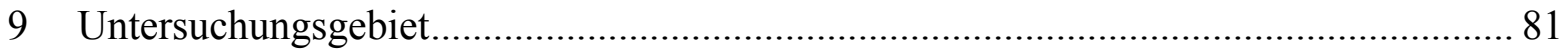

9.1 Charakterisierung der untersuchten Heckenlandschaften ..................................... 81

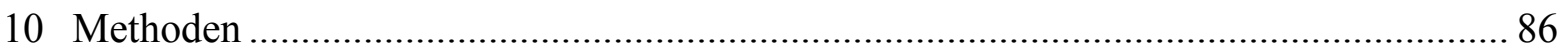

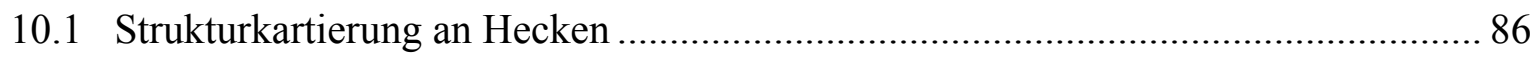

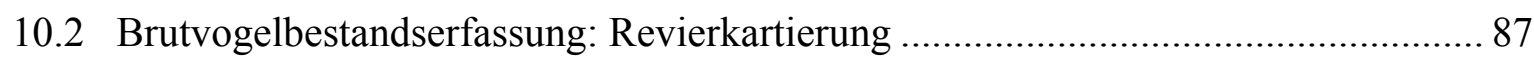

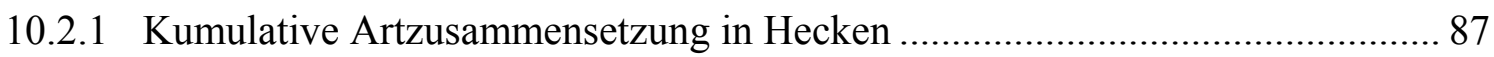

10.3 Statistik Heckenstrukturen und Brutvogelbestände ........................................... 87

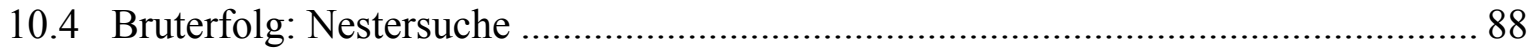

10.5 Populationsentwicklung: Berechnung der Populationsgröße im folgenden Jahr...... 90

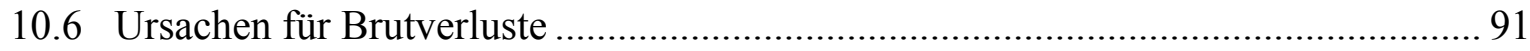

10.7 Ermittlung der Prädatoren an den Kunstnestern ................................................. 92

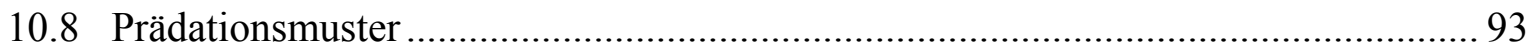

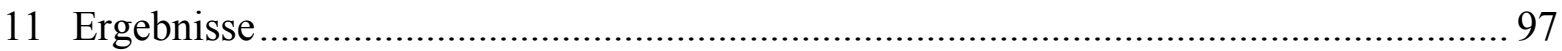




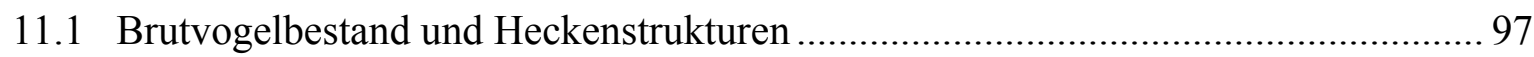

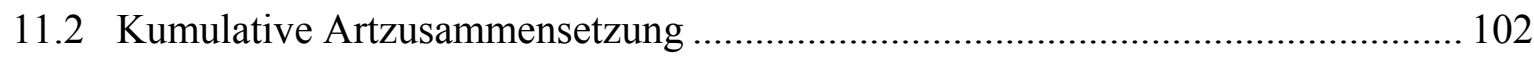

11.3 Der Einfluss von Heckenstrukturen und Landschaftselementen auf die

Abundanz von Brutvögeln in Hecken ................................................................ 103

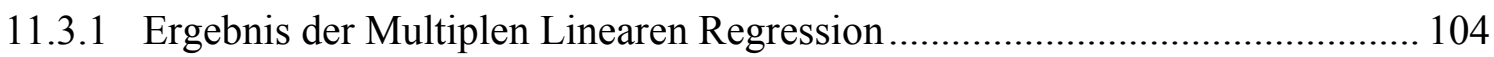

11.4 Einfluss von Heckenstrukturen auf die Dichte einzelner Vogelarten ..................... 106

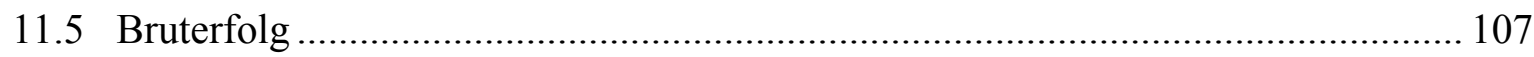

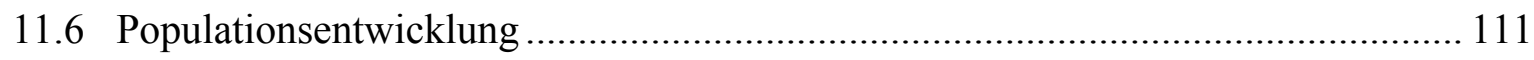

11.7 Ursachen für Brutverluste ........................................................................... 112

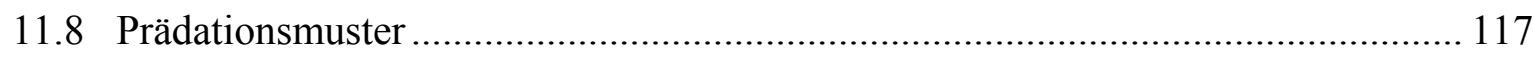

11.8.1 Welche Prädatoren treten an den Kunstnestern auf? ...................................... 117

11.8.2 Wann in der Brutzeit werden die meisten Nester ausgeraubt?...................... 117

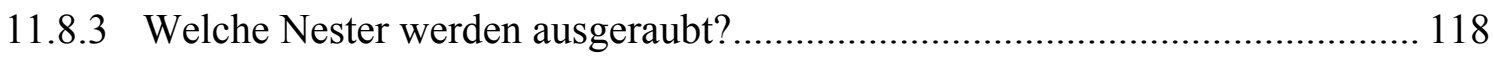

11.8.4 Werden Nester 'heckenweise' ausgeraubt? ................................................... 119

11.8.5 Welche Landschafts- und Heckenstrukturen bestimmen das

Prädationsrisiko von Kunstnestern in Hecken? ........................................... 120

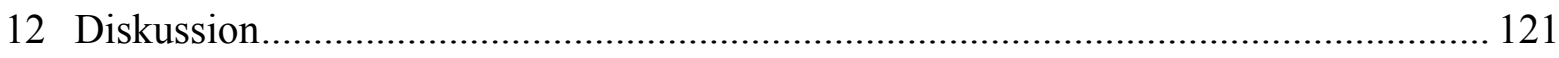

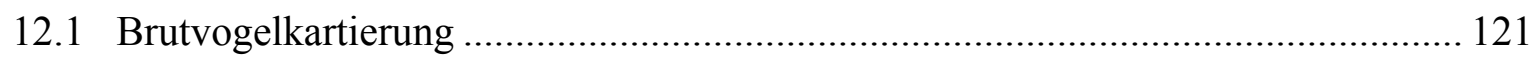

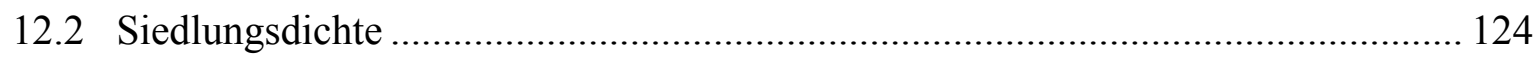

12.3 Wie konstant ist die Revierbesetzung in Hecken über die Jahre?......................... 125

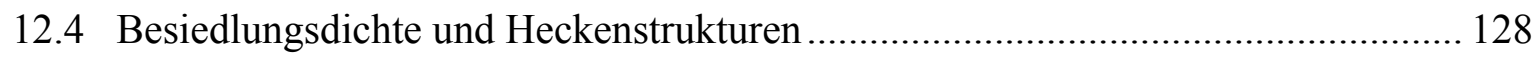

12.5 Welche Strukturen und Landschaftselemente bestimmen die Abundanz

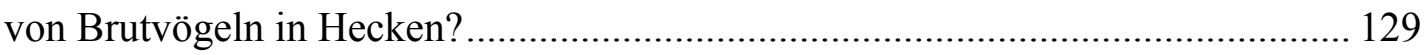

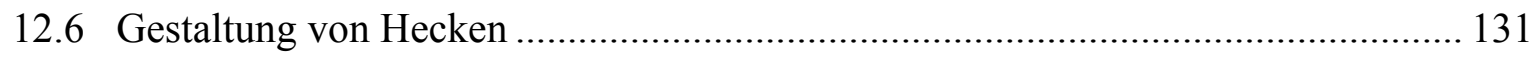

12.7 Wie viele Vogelarten muss eine aus ornithologischer Sicht 'gute'

Hecke mindestens haben?

12.8 Bruterfolg und Populationsentwicklung ...... 
12.9 Warum können Hecken für manche Arten ein Sink- und für andere Arten ein Source-Habitat sein?

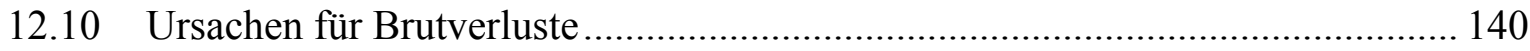

12.11 Naturschutz und Forschung in Hecken .............................................................. 144

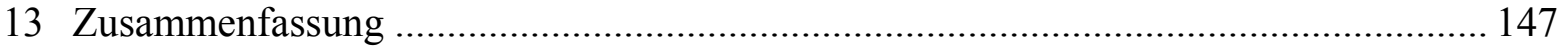

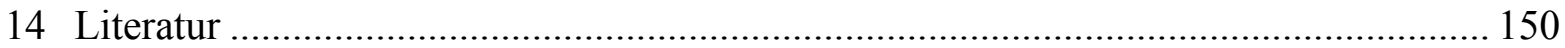

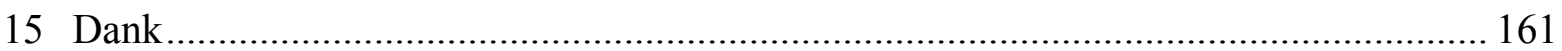

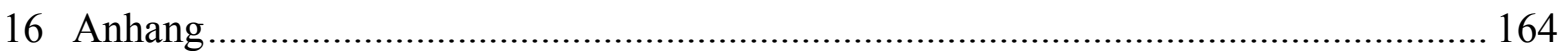

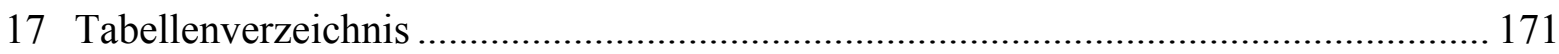

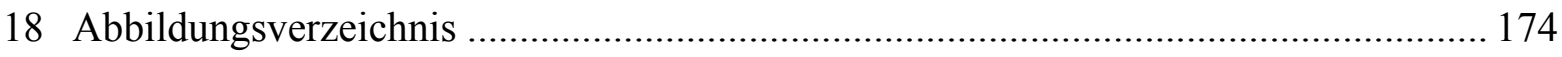

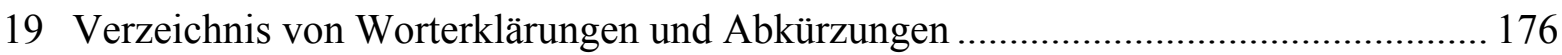




\section{Das Heckenprogramm der deutschen Vogelwarten - Netzfang und Revierkartierung zur Erfassung populationsdynamischer und reproduktionsbiologischer Aspekte in einem anthropogen geformten Lebensraum}

\section{Vorhaben und Zielsetzung}

Hecken sind wertvolle Landschaftsbestandteile, die die Struktur unserer Kulturlandschaft prägen und sowohl historisch als auch aktuell menschlicher Nutzung unterliegen (TISCHLER 1948a, Bezzel 1982, Zwölfer 1984, Streeter et al. 1985, Müller, G. 1989, Müller, J. 1990, RINGLER et al. 1997). Aus biologischer Sicht wurde die Bedeutung von Hecken als Korridore, die durch landwirtschaftliche Nutzflächen getrennte Waldhabitate miteinander verbinden, unter den Stichworten 'Biotopverbund' und 'Vernetzung' vielfältig diskutiert (MADER et al. 1986, MADER 1988, KAUle 1991a, JeDiCKE 1994). Hecken sind, unabhängig davon, zu wichtigen Lebensräumen für Tiere und Pflanzen geworden, weil es in vielen Regionen Mitteleuropas an natürlichen oder naturnahen Landschaftsbestandteilen fehlt (TISCHLER 1959, BLAB 1993, MÜHLENBERG \& SLOWIK 1997).

Das Heckenprogramm der deutschen Vogelwarten hat zum Ziel, eine umfassende Dokumentation und Bewertung von Hecken als Lebensraum für Vögel vorzulegen, um nach Möglichkeit Maßnahmen zum Schutz, zur Pflege oder zur Neuanlage von Hecken für den praktischen Naturschutz vorzuschlagen.

Neben der Erfassung der aktuell vorkommenden Arten, deren Abundanz und Abundanzveränderungen steht dabei die reproduktionsbiologische Bedeutung der Hecke im Vordergrund. Dieses geschieht durch einen erweiterten Ansatz zur Erfassung von Brutvogelpopualtionen, der nicht nur die Anzahl vorkommender Individuen, sondern auch den Bruterfolg in einem Lebensraum über einen Index aus dem Verhältnis von Jungvögeln zu Altvögeln beschreibbar macht. Zu diesem Zweck wurden nach standardisierter Methodik Netzfänge durchgeführt, um Vögel individuell zu beringen. Des Weiteren wurden die Brutpaare in den Untersuchungsgebieten kartiert und Jungvögel in Nestern oder Nistkästen konnten fakultativ beringt werden. Mit einer ebenfalls standardisierten Beschreibung der untersuchten Hecken sollte eine Bewertung besonderer Heckenstrukturen anhand der erhobenen ornithologischen Daten erfolgen. Die Dauer des Projekts war für die Jahre 1994 bis 1998 festgelegt. 
Der Aufbau von Teil I folgt der jeweils angewandten Methodik zur Beschreibung von Vogelbeständen. In Kapitel 4 werden die Ergebnisse der Brutbestandskartierungen dargestellt und diskutiert. Artenspektrum, Siedlungsdichte und die Dominanzverhältnisse der Arten sind die wichtigsten vogelkundlichen Kennwerte, die dieser Studie zu Grunde liegen. Mit der Revierkartierung wurde eine zwar aufwendige Kartierungsmethode angewandt, damit aber die Möglichkeit eröffnet, eine sehr fundierte Datengrundlage zum Vergleich mit den Ergebnissen des Netzfangs und anderen Studien zu erhalten. Nach FLADE (1994) können mit der Revierkartierung das Artenspektrum „nahezu vollständig (mindestens 90 \%) und die Häufigkeitsverhältnisse in erträglichen Fehlergrenzen (weniger 25 \% bei häufigen Kleinvögeln)“ angegeben werden.

Im 5. Kapitel wird mit dem Fangprogramm der methodisch wichtigste Teil der Arbeit behandelt. In die jeweiligen Abschnitte wird kurz eingeführt, methodische Erweiterungen und die Ergebnisse werden abschnittsweise dargestellt und diskutiert. Durchführung und Durchführbarkeit des standardisierten Netzfangs werden dargestellt und im Hinblick auf eine Anwendung im Integrierten Monitoring für Singvogelpopulationen (IMS, BAIRLEIN et al. 2000) bewertet.

Der Frage nach der Bedeutung von Hecken für Vögel kann man sich generell auf zwei verschiedenen Wegen nähern: Einerseits, indem man den Lebensraum von den Arten ausgehend beschreibt und versucht, folgende Fragen zu beantworten:

Wie groß ist das Artenspektrum in Hecken, welche Arten umfasst es, welche Arten sind am häufigsten, welche typischen Arten kommen in Hecken vor, welche seltenen Arten siedeln in Hecken, welche Arten sind auf Hecken angewiesen und wie gut können die betreffenden Arten in den Hecken überleben und sich reproduzieren? Diese Herangehensweise führt über den Artenschutz zum Biotopschutz oder zur Biotoppflege.

Der andere Weg geht von der Bedeutung des Lebensraums aus und versucht die Frage zu beantworten, in welcher Hecke die meisten Vogelarten siedeln, die höchste Vogeldichte oder die höchste Diversität erreicht wird. Dieser Ansatz führt über einen gezielten Biotopschutz zum Artenschutz. Ziel könnte das Leitbild einer 'optimalen Hecke' für Vögel sein.

In diesem Teil der Arbeit werden beide Ansätze verfolgt. Im Einzelnen wird der Frage nachgegangen, wie groß die Unterschiede zwischen den einzelnen Hecken sind. Dazu werden die Fangzahlen verglichen und die durchschnittliche Anzahl von Fänglingen sowie Minima und Maxima beschrieben. Auf der Basis der Fangsummen einer repräsentativen Auswahl von Heckenvögeln, wird die Bedeutung von bestimmten Heckenstrukturen analysiert und in 
einem zweiten Schritt wird die Bevorzugung einzelner Heckenstrukturen durch die Arten dargestellt.

Das Aussterben und die Arealausweitung, der Bestandsrückgang und die Bestandszunahme werden durch die Bestandsdynamik der Arten beschreibbar. Insofern ist die Beobachtung von Bestandsverläufen ein wichtiges Instrument des Artenschutzes. Das Heckenprogramm der deutschen Vogelwarten dient diesem Ziel mit der standardisierten Erfassung von Vogelpopulationen. Fangsummen und Bestandsverläufe werden im Sinne eines klassischen Monitorings für Jung- und Altvögel beschrieben. Abundanzveränderungen einzelner Arten werden in ihrer Relation zum Gesamtfang errechnet, um die Bedeutung der Bestandsveränderungen unter dem Einfluss des allgemeinen Fangerfolgs abschätzen zu können.

Für den ökologischen Wert eines Habitats ist der Bruterfolg der Arten von großer Bedeutung. Aus dem Verhältnis von Jung- zu Altvögeln wird der Bruterfolg jeden Jahres indiziert. Anhand eines so erhobenen ,relativen Bruterfolgs“ lassen sich bereits viele Erklärungen oder Hypothesen für die Entwicklung von Brutvogelbeständen und bestandsverändernde Faktoren ableiten (BAIRLEIN et al. 2000). Mögliche Beziehungen zwischen „relativem Bruterfolg“ der Bestandsentwicklung der Arten werden aufgezeigt und diskutiert. Faktoren die den Bruterfolg von Arten beeinträchtigen können und deren potentielle Folgen werden benannt.

Die Anzahl von Wiederfängen, Vögel die mehrmals in einer Brutsaison in einer Hecke gefangen werden, sind mit großer Wahrscheinlichkeit territoriale Individuen und bieten neben der Kartierung eine zweite Möglichkeit zur Abschätzung des Brutbestandes. Der Anteil der Wiederfänge am Gesamtfang wird beschrieben und der Frage nachgegangen, ob die Entwicklung der Wiederfänge über die Jahre mit den Ergebnissen der Bestandsdynamik aller Fänglinge in Übereinstimmung steht oder ob Vogelpopulationen in Hecken einer eigenen Dynamik unterliegen.

Der Bestand von Vögeln in einem Habitat unterliegt auch zur Brutzeit ständigen Schwankungen, weil Individuen abwandern oder sterben, bzw. einwandern und Reviere besetzten oder unverpaart bleiben (BAIRLEIN 1978). Die allgemeine Phänologie von Fänglingen läßt dabei nur in kleinen Zeitfenstern Rückschlüsse auf die Phänologie der Brutpopulation zu. Anhand von Wiederfängen lassen sich Revierinhaber und Durchzügler unterscheiden und Fragen nach dem Eintreffen der Revierinhaber in die Bruthabitate, deren Anteil am Gesamtfang in Abhänigigkeit der Dekade und die mittlere Zeitspanne zwischen der Beringung und dem Wiederfang beantworten. 
In Kapitel 6 werden Resultate aus Kartierungen und Netzfängen zusammengeführt und verglichen. Unterschiede und Übereinstimmungen werden anhand der Biologie der Arten diskutiert.

Teil I der Arbeit schließt mit einer Zusammenfassung. Anhang und Literaturangaben finden sich am Ende der Gesamtarbeit. Fachspezifische Ausdrücke und Abkürzungen sind in einem Glossar aufgeführt und erklärt. 


\section{Die Untersuchungsgebiete}

Die Verteilung der Probeflächen innerhalb Deutschlands ergibt sich aus der Lage der Wohnorte der jeweiligen Bearbeiter (Abb. 1).

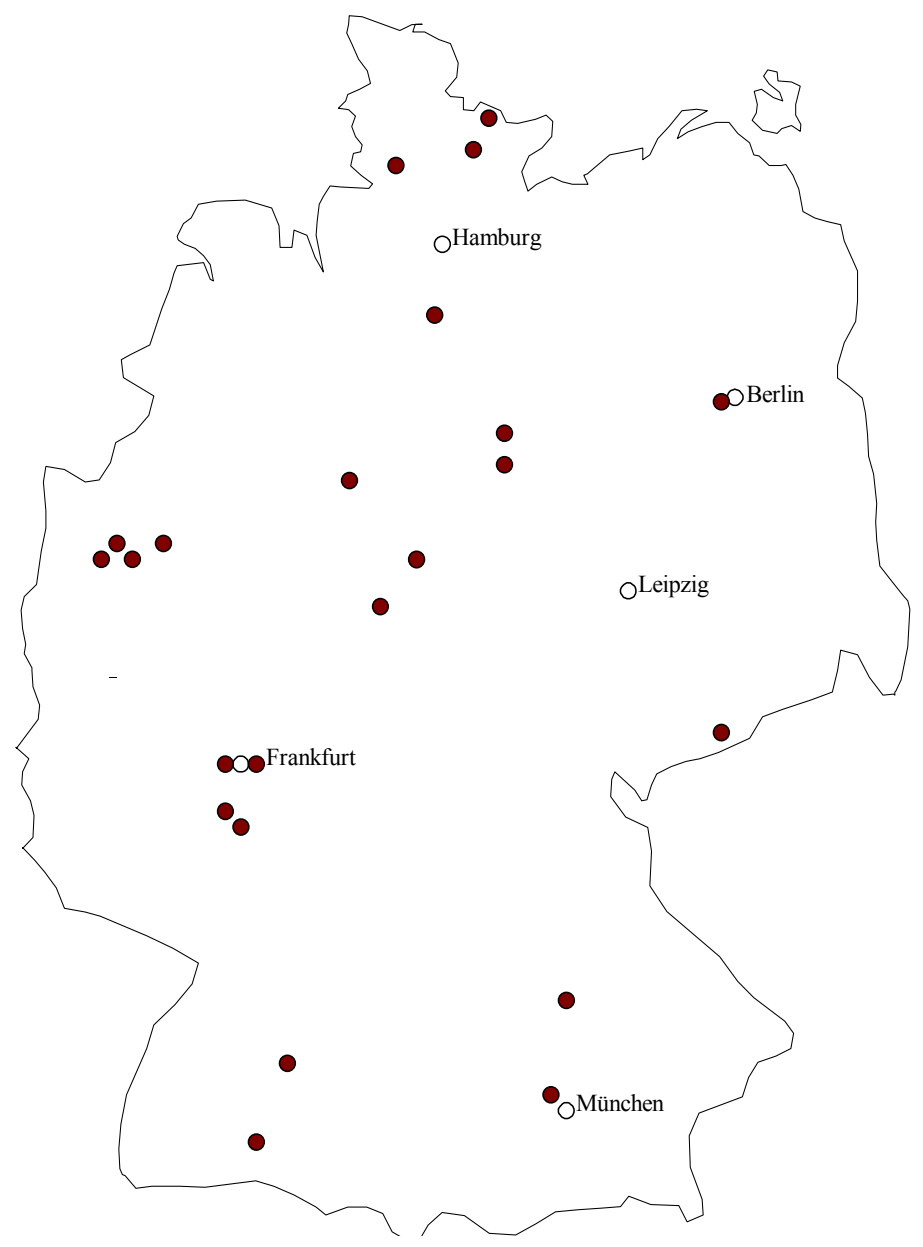

Abb. 1: Geographische Lage der Untersuchungsflächen

Die Untersuchungsflächen der Mitarbeiter sind von Norden nach Süden betrachtet weitgehend gleichmäßig über die Fläche der Bundesrepublik Deutschland verteilt. Im Westen sind die Bundesländer Saarland und Rheinland-Pfalz nicht vertreten, die Neuen Bundesländer sind nur durch je ein Untersuchungsgebiet in Sachsen und Berlin repräsentiert. Die geographische Lage der Probeflächen stellt eine insgesamt gleichmäßig verteilte Stichprobe typischer Heckenstandorte in Deutschland dar.

Es wurden 33 Hecken bearbeitet, nicht alle über die gesamte Laufzeit. Auf zwei Flächen wurden keine Brutvogelkartierungen durchgeführt. Zum Teil sind von einigen Bearbeitern mehrere Heckenabschnitte beschrieben, die Fang- und Kartierungsergebnisse jedoch nicht spezifisch zugeordnet worden, so dass für diese Fälle nur jeweils eine Hecke in Tab. 1 
angegeben ist. Von 14 Mitarbeitern wurden 23 Hecken über den gesamten Zeitraum des Heckenprogramms bearbeitet.

Tab. 1: Die ehrenamtlichen Mitarbeiter und Orte der Untersuchungsgebiete, nach PLZ sortiert.

\begin{tabular}{|l|c|l|l|}
\hline \multicolumn{1}{|c|}{ Mitarbeiter } & PLZ & \multicolumn{1}{|c|}{ Ort } & \multicolumn{1}{c|}{ Bundesland } \\
\hline Schlegel, S. \& J. & 9456 & Annaberg & Sachsen \\
Fischer, S. & 10115 & Berlin & Berlin \\
Hoff, H.-J. & 21255 & Tostedt & Niedersachsen \\
Grajetzy, B. & 23845 & Wakendorf & Schleswig-Holstein \\
Schwarze, H. & 24211 & Pohnsdorf & Schleswig-Holstein \\
Martens, S. & 25524 & Itzehoe & Schleswig-Holstein \\
Hadasch, J. & 32051 & Herford & Nordrhein-Westfalen \\
Giessler, H. & 34270 & Schauenbg.-Elgersh. & Hessen \\
Barkow, A. & 37083 & Göttingen & Niedersachsen \\
Greve, K. & 38124 & Braunschweig & Niedersachsen \\
Schemmel, H. & 38547 & Calberlah & Niedersachsen \\
Giese, J. \& Kannhäuser, H. & 40724 & Hilden & Nordrhein-Westfalen \\
Patt, H. W. & 42349 & Wuppertal & Nordrhein-Westfalen \\
Vohwinkel, R. & 42553 & Velbert & Nordrhein-Westfalen \\
Heppe, W. & 44287 & Dortmund & Nordrhein-Westfalen \\
Eidam, U./ Untermain & 60325 & Frankfurt (M) & Hessen \\
Lang, K.-H. & 60437 & Frankfurt (M) & Hessen \\
Semrau, V. & 64584 & Biebesheim & Hessen \\
Diry, H. & 64665 & Alsbach Hähnlein & Hessen \\
Laich, W. & 70469 & Stuttgart & Baden-Württemberg \\
Neumann, M. A. & 78647 & Trossingen & Baden-Württemberg \\
Hollenbach, H. & 85123 & Karlskron-Mändlfeld & Bayern \\
Hausmann, S. & 85764 & Oberschleißheim & Bavern \\
\hline
\end{tabular}

Die Erhebung der Daten dieser Studie erfolgte durch die ehrenamtlich beteiligten Mitarbeiter am Heckenprogramm der deutschen Vogelwarten (Tab. 1). Das Heckenprogramm der deutschen Vogelwarten ist ein gemeinschaftliches Projekt der Vogelwarte Helgoland, der Vogelwarte Hiddensee und der Vogelwarte Radolfzell. Die Organisation lag beim Institut für Vogelforschung 'Vogelwarte Helgoland' in Wilhelmshaven. 


\section{Heckenbeschreibung}

Die Auswahl der jeweils zu untersuchenden Hecken war den Bearbeitern überlassen. Vorgegeben war lediglich die Charakterisierung einer Hecke als ,jede gehölzartige lineare Struktur, die mehr oder weniger isoliert in der Kulturlandschaft vorkommt, [...]“ (BAIRLEIN 1996b). Waldsäume, Feldgehölze oder Einzelbüsche sollten nicht untersucht werden, wohingegen jede Hecke unabhängig von ihrer Ausprägung und landschaftlichen Umgebung bearbeitet werden konnte.

In das bewusst sehr breit angelegte Spektrum fielen Hecken mit 150 bis über $1500 \mathrm{~m}$ Länge, frisch angelegte (Benjes-) Hecken und über 50 Jahre alte Feldhecken, sowohl Gartenhecken als auch Hecken auf Lesesteinwällen, Hecken an ehemals bewirtschafteten Teichen, Knicks oder Anpflanzungen an Parks oder im Bereich von Straßen und Parkplätzen. Die Mehrzahl der im Heckenprogramm untersuchten Hecken sind typische Feldhecken der halboffenen Kulturlandschaft.

Zur Beschreibung der Hecken diente ein Heckenerfassungsbogen, auf dem die wichtigsten Strukturmerkmale der Hecken von den Bearbeitern notiert wurden. Diese vereinheitlichte Beschreibung der Hecken bietet die Grundlage für den Vergleich der Untersuchungsgebiete. Im Einzelnen wurden Angaben zu folgenden Parametern erfasst:

- Lage: Ort, Koordinaten, Meereshöhe, Hangneigung und Ausrichtung der Hecke

- Maße: Höhe, Breite (Durchschnitts- und Maximalwert) und Länge

- Alter: Angabe einer von sieben Kategorien

- Heckentyp: 5 Auswahlmöglichkeiten von 'Strauchhecke' bis 'Baumreihe ohne Sträucher'

- Heckendichte: 'geschlossen', 'mit einzelnen Lücken' oder 'stark lückig'

- Gehölzarten: Angabe einer von drei Kategorien und (separat) der Hauptgehölzarten

- Krautschicht: Angabe einer von drei Kategorien und der dominierenden Arten

- Nutzung der angrenzenden Flächen: Ankreuzmöglichkeiten für jede Heckenseite

- Beeinträchtigungen: Ankreuzmöglichkeiten für jede Heckenseite

- Bemerkungen

Veränderungen oder Aktualisierungen wurden jedes Jahr abgefragt und bei der Auswertung berücksichtigt. 


\section{Brutvogelbestände}

Die Brutvogelkartierung gibt einen Überblick über das Arteninventar sowie die Dichte, Dominanz und Verteilung einzelner Arten. Die Ermittlung des Brutvogelbestandes dient als grundlegende Vergleichsbasis zu den Ergebnissen des Fangprogramms in dieser Studie und zu Untersuchungen anderer Autoren, die Vogelbestände in Heckenlandschaften vorzugsweise mittels Kartierung erfassen.

\subsection{Methode: Revierkartierung und Linientaxierung}

Der Brutvogelbestand in den Hecken wurde von April bis Juli, zwei bis dreimal je Monat, durch langsames Ab- oder Durchschreiten der Untersuchungsflächen ermittelt. Den Vorgaben zur Durchführung der Revierkartierung folgend (BIBBY et al. 1992) wurden alle revieranzeigenden Beobachtungen an Vögeln notiert. In einer Brutperiode wurden mindestens 10 morgendliche Begehungen durchgeführt, die Ergebnisse in Tagesprotokollen festgehalten und nach Abschluss der Feldarbeit in Artkarten zusammengeführt. Die auf diese Weise erhobene Anzahl von unterscheidbaren Territorien ('Papierreviere') wurden als Maß für die Summe der Brutpaare akzeptiert. Der Brutbestand wurde von den Bearbeitern in den Kategorien 'Brutpaare sicher' und 'Brutpaare fraglich' angegeben. Dadurch kann zwischen einem Mindestbrutbestand und einer Anzahl zumindest temporär vorhandener Reviere unterschieden werden.

\subsection{Arten und Abundanzen des Brutvogelbestandes}

Ergebnis: Bei der Erfassung der Brutvogelbestände konnten in den Hecken 68 Arten festgestellt werden. 59 Arten wurden mit mindestens einem sicheren Brutpaar in einer Hecke in einem Jahr erfasst (Tab. 2). Bei neun weiteren Arten war der Brutstatus fraglich. Es waren 51 Singvogelarten mit mindestens einem sicheren Brutpaar in den untersuchten 33 Hecken vertreten.

Die Ringeltaube hatte mit insgesamt über 100 Revieren die höchste Abundanz aller Nichtsingvögel. Von den drei Glattfußhühnern war der eingebürgerte Fasan am häufigsten. Rebhuhn und Wachtel konnten nur an wenigen Hecken festgestellt werden. Es konnte kein Greifvogel mit einem sicher festgestellten Brutpaar in den untersuchten Hecken nachgewiesen werden. 
Tab. 2: Sichere und fragliche Brutpaare (BP) sowie der Anteil fraglicher BP (\%) der im Heckenprogramm über fünf Jahre erfassten Reviere in 33 Hecken mit $>10$ BP. BP si, BP fr = sichere bzw. fragliche Brutpaare, \% fr = Anteil fraglicher Brutpaare, * = Vögel die (methodisch bedingt) nicht durch Netzfang nachgewiesen werden konnten.

\begin{tabular}{|c|c|c|c|c|}
\hline & ART & BP si & BP fr & $\%$ fr \\
\hline 1 & Mönchsgrasmücke & 300 & 57 & 16 \\
\hline 2 & Zilpzalp & 294 & 53 & 15,3 \\
\hline 3 & Amsel & 265 & 42 & 13,7 \\
\hline 4 & Gartengrasmücke & 194 & 49 & 20,2 \\
\hline 5 & Goldammer & 188 & 47 & 20 \\
\hline 6 & Heckenbraunelle & 170 & 55 & 24,4 \\
\hline 7 & Kohlmeise & 158 & 28 & 15,1 \\
\hline 8 & Buchfink & 152 & 24 & 13,6 \\
\hline 9 & Dorngrasmücke & 125 & 37 & 22,8 \\
\hline 10 & Fitis & 109 & 27 & 19,9 \\
\hline 11 & Rotkehlchen & 108 & 46 & 29,9 \\
\hline 12 & Blaumeise & 102 & 18 & 15 \\
\hline 13 & Ringeltaube & 83 & 27 & 24,5 \\
\hline 14 & Sumpfrohrsänger & 82 & 33 & 28,7 \\
\hline 15 & Grünling & 77 & 28 & 26,7 \\
\hline 16 & Singdrossel & 73 & 29 & 28,4 \\
\hline 17 & Feldsperling & 70 & 26 & 27,1 \\
\hline 18 & Gelbspötter & 65 & 23 & 26,1 \\
\hline 19 & Klappergrasmücke & 57 & 16 & 21,9 \\
\hline 20 & Zaunkönig & 49 & 23 & 31,9 \\
\hline 21 & Neuntöter & 46 & 8 & 14,8 \\
\hline 22 & Star & 37 & 5 & 11,9 \\
\hline 23 & Elster* & 25 & 4 & 13,8 \\
\hline 24 & Nachtigall & 22 & 5 & 18,5 \\
\hline 25 & Wacholderdrossel & 19 & 8 & 29,6 \\
\hline 26 & Fasan* & 18 & 6 & 25 \\
\hline 27 & Girlitz & 17 & 4 & 19 \\
\hline 28 & Weidenmeise & 13 & 10 & 43,5 \\
\hline 29 & Gimpel & 12 & 8 & 40 \\
\hline 30 & Haussperling & 11 & 2 & 15,4 \\
\hline 31 & Rebhuhn* & 10 & 2 & \\
\hline 32 & Kuckuck & 10 & & \\
\hline 33 & Rabenkrähe* & 10 & & \\
\hline 34 & Sumpfmeise & 8 & 12 & \\
\hline
\end{tabular}

\begin{tabular}{|c|c|c|c|}
\hline & ART & BP si & BP fr \\
\hline 35 & Stieglitz & 7 & 12 \\
\hline 36 & Schwanzmeise & 7 & 8 \\
\hline 37 & Gartenrotschwanz & 7 & 6 \\
\hline 38 & Bachstelze & 6 & 3 \\
\hline 39 & Hausrotschwanz & 6 & \\
\hline 40 & Buntspecht & 5 & 5 \\
\hline 41 & Trauerschnäpper & 5 & 3 \\
\hline 42 & Grauschnäpper & 5 & 2 \\
\hline 43 & Kernbeißer & 5 & 2 \\
\hline 44 & Sprosser & 5 & \\
\hline 45 & Rohrammer & 4 & 3 \\
\hline 46 & Beutelmeise & 4 & 2 \\
\hline 47 & Turteltaube & 4 & \\
\hline 48 & Wachtel* & 4 & \\
\hline 49 & Eichelhäher & 4 & \\
\hline 50 & Gartenbaumläufer & 3 & 5 \\
\hline 51 & Pirol & 3 & 1 \\
\hline 52 & Bluthänfling & 2 & 12 \\
\hline 53 & Kleiber & 2 & 4 \\
\hline 54 & Baumpieper & 2 & 2 \\
\hline 55 & Tannenmeise & 2 & \\
\hline 56 & Feldschwirl & 1 & 7 \\
\hline 57 & Schlagschwirl & 1 & 4 \\
\hline 58 & Misteldrossel & 1 & 2 \\
\hline 59 & Grünspecht* & 1 & \\
\hline 60 & Haubenmeise & & 4 \\
\hline 61 & Sperber & & 2 \\
\hline 62 & Sperbergrasmücke & & 2 \\
\hline 63 & Kleinspecht & & 1 \\
\hline 64 & Schafstelze & & 1 \\
\hline 65 & Teichrohrsänger & & 1 \\
\hline 66 & Waldkauz & & 1 \\
\hline 67 & Erlenzeisig & & 1 \\
\hline 68 & Waldlaubsänger & & 1 \\
\hline
\end{tabular}

Mönchsgrasmücke, Zilpzalp und Amsel waren die häufigsten Brutvögel in den untersuchten Hecken. 12 Arten kamen mit mehr als 100 Brutpaaren vor. Einige Arten, die durch ein Fangprogramm nicht erfasst wurden, konnten anhand der Revierkartierung als Brutvogel in Hecken nachgewiesen werden. Es handelte sich hierbei ausschließlich um Rabenvögel und Nicht-Singvögel.

Diskussion: Unter den häufigsten 10 Arten waren nur Goldammer und Dorngrasmücke als typische Vertreter halboffener Landschaften vertreten (vgl. BLAB 1993). Zudem war der Anteil fraglicher Reviere bei diesen Arten mit $20 \%$ relativ hoch, obwohl beide Arten durch Reviergesang und Territorialverhalten sehr auffällig sind. Da sowohl Dorngrasmücke als auch 
Goldammer vor allem in den Randbereichen von Hecken oder in angrenzenden Strukturen brüten (Vorliebe für Gräben bei Erstbruten der Goldammer, LiLle 1996), sind einige Reviere von den Bearbeitern 'nur halb' den Hecken selbst zugeordnet und als 'Brutpaar fraglich' eingestuft worden.

Der Neuntöter war mit 46 Brutpaaren vertreten. Wenn man hypothetisch davon ausgeht, dass der Neuntöter dort wo er auftritt in jedem Jahr erscheint, so verbleiben rein rechnerisch neun Hecken, in denen der Neuntöter Brutvogel war. Diese einst so charakteristische Art für Landschaftstypen mit halboffenen Gehölzstrukturen brütete also nicht einmal mehr in einem Drittel der untersuchten Hecken regelmäßig.

Eine weitere Art, die bei der Betrachtung von Hecken besonderes Interesse verdient, ist der Feldschwirl, der in Baden-Württemberg in der Roten Liste als 'gefährdet' geführt wird (JEDICKE 1997). Diese Art brütet nicht direkt in Hecken, aber in gehölzfreien Lücken dazwischen, wo z.B. vorjährige Stauden in flächenhaft ausgeprägter Krautschicht stehen bleiben können (eig. Beob.). Bei der Erfassung der Brutpaare ist die Art wahrscheinlich unterrepräsentiert, weil die Reviere nicht der Hecke selbst zugeordnet wurden. Ähnliches kann für den Bluthänfling zutreffen, der häufig in dichten Einzelbüschen brütet (BEZZEL 1993, eig. Beob.) oder Nahrungshabitate aufsucht, die vom Brutort getrennt liegen können (EYBERT et al. 1995). Beide Arten weisen eine hohe Anzahl 'fraglicher Brutpaare' auf. Der Bestand dieser Arten sollte bei Untersuchungen an Hecken in Zukunft genau beobachtet werden, weil sowohl Feldschwirl als auch Bluthänfling in sechs Bundesländern über die Jahre 1970-1994 um mehr als $20 \%$ abgenommen haben (WITT et al. 1996) und nach CES-Erhebungen in UK stark rückläufig sind (BALMER \& PEACH 1996).

Darüber hinaus ist der Bestand an Brutvögeln in Hecken durch hochfrequentes Vorkommen allgemein verbreiteter Arten geprägt, die zum einen der Gilde waldbewohnender Singvögel zuzuordnen sind (z. B. Mönchsgrasmücke, Singdrossel, Buchfink) und zum anderen eine große Überschneidungsbreite mit den Leitarten von Friedhöfen und Parks (z. B. Gartenrotschwanz, Gelbspötter, Girlitz, Grauschnäpper) aufweisen (vgl. FLADE 1994). Vor allem das Spektrum 'lebensraumholder Arten' und 'steter Begleiter', die von FLADE (1994) für oben genannte Lebensräume ermittelt worden sind, findet sich in den untersuchten Hecken wieder: 'Lebensraumhold' sind in Friedhöfen z.B. Amsel, Grünling, Ringeltaube mit jeweils 100 \%; 'stete Begleiter' in Parks (80-100 \%) sind Star, Amsel, Kohlmeise, Buchfink, Blaumeise, Grünling, Zilpzalp, Rotkehlchen, Mönchsgrasmücke, Singdrossel, Fitis und Aaskrähe.

Über alle Hecken betrachtet ist die Anzahl erfasster Reviere bei Gartenrotschwanz und Turteltaube sehr niedrig. Beide Arten sind als Bewohner halboffener Agrarlandschaften 
beschrieben (z.B. BEZZEL 1982, FlADE 1994) und in den Hecken als Brutvogel zu erwarten. Methodische Fehler bei der Erfassung dieser Arten sind unwahrscheinlich, weil in den vorhandenen Nistkästen regelmäßige Kontrollen durchgeführt worden sind, wobei weitere Bruten von Gartenrotschwanz aufgefallen wären. Turteltauben haben einen sehr charakteristischen Gesang.

Das Artenspektrum deckt sich weitgehend mit Ergebnissen anderer Untersuchungen an Hecken und zeigt Überschneidungen mit Vogelbeständen in wenigstens teilweise gehölzbestandenen Agrarlandschaften (z.B. PUChSteIn 1980, Oelke 1985, GaßMANN \& GlÜCK 1988). Hervorzuheben ist das Fehlen von Arten, die aufgrund ihrer Habitatansprüche in den untersuchten Lebensräumen zu erwarten wären. Neben den auch im Heckenprogramm festgestellten Gold-und Rohrammern konnte KUJAWA (1997) in Westpolen mit Grauammer und Ortolan zwei weitere Ammern in hoher Abundanz finden. Der Ortolan erreicht dort eine Dichte von 4,5 Brutpaaren je Hektar. Die Grauammer gehört zu den dominanten Arten der agrarisch genutzten Kulturlandschaft um Posen. In der an Alleen, Gehölzschutzpflanzungen (sog. 'Shelter Belts', vgl. MüHLENBERG \& SLOWIK 1997) und kleinen Waldinseln (Mittelwert: 1,5 ha Fläche) durchgeführten Untersuchung wurden darüber hinaus Raubwürger, Braunkehlchen und auch Steinkauz und Wendehals nachgewiesen (KuJAWA 1997).

Die angesprochenen Untersuchungsflächen in Westpolen zeigen geographisch und klimatisch eine enge Verbindung mit den im Heckenprogramm bearbeiteten Probeflächen: Deshalb ist der Verlust dieser Arten, die in Deutschland ebenfalls verbreitet sind, bei einer Bewertung von Heckenstrukturen mit zu berücksichtigen. Eine ausführlichere Diskussion zu diesem Punkt findet sich unter 12.1.

Insgesamt umfasst das Artenspektrum der im Heckenprogramm erfassten Brutvögel vor allem eurytope Arten, die sich aufgrund ihrer Anpassungsfähigkeit bereits in vielen vom Menschen geschaffenen Lebensräumen etabliert haben.

Einige Arten zeigen einen auffällig hohen Anteil von Revieren, die als fraglich eingestuft werden mussten (Tab. 2). Die Ursachen sind zumeist in arttypischen Besonderheiten zu suchen. Sumpfrohrsänger und Dorngrasmücke, die unverkennbares Verhalten am Brutplatz zeigen, kehren erst relativ spät im Jahr in die Brutgebiete zurück und setzen bei ungünstigen Witterungsverhältnissen und in bestimmten Phasen des Brutgeschäfts mit der Gesangsaktivität aus. Hier kann möglicherweise die Anzahl der Kontakte bei der Berechnung der Anzahl der Papierreviere nicht ausreichend für die Annahme eines sicheren Brutpaares sein (Kontakt bei $\geq 50 \%$ aller Begehungen, in denen die Art im Brutgebiet zu erwarten ist), so 
dass sich die Bearbeiter für die Angabe von 'Brutpaar fraglich' entschieden. Bei der Singdrossel kann der frühe Beginn des Gesanges der Männchen, was den Jahres- als auch den Tagesgang (Höhepunkt bereits 60 Minuten vor Sonnenaufgang, MELDE 1991) anbetrifft sowie die kurze Gesangsdauer in den Morgenstunden (z.B. BEZzEL 1993), eine Ursache für die hohe Anzahl der fraglichen Reviere sein. Zwischen den beiden Jahresbruten und nach jeder abgebrochenen Brut können Revierverlagerungen stattfinden, auch dahin gehend dass Singdrosseln in der Brutzeit nach ein oder zwei missglückten Brutversuchen aus der Hecke auswandern (eig. Beob.), wie KREBS (1971) das für Kohlmeisen beobachten konnte.

Mit knapp $30 \%$ hat das Rotkehlchen den höchsten Anteil fraglicher Reviere unter den häufigsten Arten, obwohl der Gesang sehr auffällig und der Vogel durch häufiges 'Tixen' vielfach wahrnehmbar ist. Der Grund kann darin liegen, dass viele Rotkehlchen die Hecken zwar über das Jahr hinweg nutzen, dort aber nicht zur Brut schreiten oder diese Lebensräume sogar zur Brutzeit verlassen (vgl. geringe Fangzahlen adulter Rotkehlchen in Abb. 10). Gesang oder auffälliges Verhalten, das als revieranzeigend verstanden werden könnte, wird an Rotkehlchen bereits ab März in den Hecken beobachtet. Ab Mitte Juni treten Jungvögel in den Hecken auf und es entsteht der Eindruck, dass diese auch tatsächlich in der Hecke erbrütet worden sind. Das trifft für viele Hecken jedoch nicht zu und zeigt sich darin, dass im Mai keine adulten Rotkehlchen festzustellen sind, Nestfunde und Beobachtungen fütternder Altvögel und ähnliche Hinweise auf Brutvorkommen fehlen. Ausnahmen bilden waldähnliche Hecken und mitunter alte Knicks in Schleswig-Holstein und Niedersachsen, die aber als doppelte Hecken (Redder) angelegt sein müssen, um als Bruthabitat für Rotkehlchen geeignet zu sein (GRAJETZKY 1993, 2000, PUChSTEIN 1980).

Der Anteil 'fraglicher Brutpaare' bei Gartengrasmücke und Heckenbraunelle ist vergleichsweise hoch, dabei zeigen beide Arten ihre Reviere durch intensiven Gesang an und die Aufenthaltsorte sind in der Brutzeit deutlich revierbezogen. Es ist denkbar, dass eine hohe Anzahl von Nichtbrütern (v.a. Heckenbraunelle) und noch lange nachwirkender Durchzug zu der Annahme von Brutpaaren führt, weil auch kurzzeitig anwesende Individuen beider Arten ausgeprägtes Territotialverhalten zeigen können.

Höhlenbrüter werden vollständig erfasst, sofern sie künstliche Nisthöhlen beziehen (Kohlund Blaumeise und Star). Beim Feldsperling erleichtert die Kontrolle über Nistkästen die Feststellung von Brutpaaren, die Art ist ansonsten sehr schwer zu kartieren (FLADE 1994). Entsprechend ist der Anteil fraglicher Reviere sehr hoch. Bei allen weiteren Höhlenbrütern, die nicht zu den regelmäßigen Benutzern von Nistkästen gehören, sind die Anteile 'fraglich' 
gebliebenen Reviere sehr hoch (Weiden- und Sumpfmeise, Kleiber, Gartenrotschwanz, Buntspecht, u.a.).

Allgemein fallen Unterschiede bei der Einstufung von 'sicheren' und 'fraglichen' Brutpaaren auf. Einige Bearbeiter waren besonders zurückhaltend mit der Bezeichnung 'Brutpaar sicher' sind und gaben diesen Status erst nach eindeutigen Beobachtungen zum Brutverhalten oder nach Nestfunden an. Im Rahmen des Heckenprogramms wurde keine allgemeine Schulung zur Homogenisierung des Vorgehens bei der Revierkartierung durchgeführt. Neben individuellen Unterschieden der Fähigkeiten von Bearbeitern kann somit auch die Art und Weise der Durchführung der Revierkartierung je nach Mitarbeiter verschieden sein und mit einem systematischen Fehler behaftet sein (s.a. Berthold 1976, OelKe 1980, BiBBY et al. 1992, GNIELKA 1992).

\subsection{Besiedlungsdichte und Diversität von Brutvögeln in Hecken}

Hecken werden je nach Ausprägung und Umgebung von verschiedenen Arten in unterschiedlicher Dichte und Diversität besiedelt. Im Folgenden soll der Frage nachgegangen werden, in welcher Größenordnung diese ökologischen Kenngrößen für den Lebensraum Hecke liegen und inwieweit diese miteinander zusammen hängen, um daraufhin Rückschlüsse auf die Vogelfauna in Hecken ziehen und diskutieren zu können.

Methoden: Die jeweils für die Hecken erhobenen Angaben für Brutpaardichte und Diversität sind gemittelte Werte aus den jeweiligen Untersuchungsjahren. Mit der Brutpaardichte (Abundanz) wird beschrieben, wieviele Individuen auf einer Fläche vorkommen. Diversität wurde nach dem Shannon-Wiener-Index berechnet (SCHAEFER 1992, MÜHLENBERG 1993). Diversität ist ein relatives Maß für die Artenzahl und Abundanzverteilung einzelner Arten. Zur Berechnung siehe Kapitel 10.3 Statistik Heckenstrukturen und Brutvogelbestände.

E: Die Besiedlungsdichte von 27 untersuchten Hecken liegt im Mittel bei 53,5, der Median beträgt 48,5 BP/km. Die Spanne umfasst 5 bis maximal etwa 180 Brutpaare. Über $50 \%$ der Hecken weisen eine Besiedlungsdichte von 20-90 Brutpaaren je Kilometer auf (Abb. 2). 

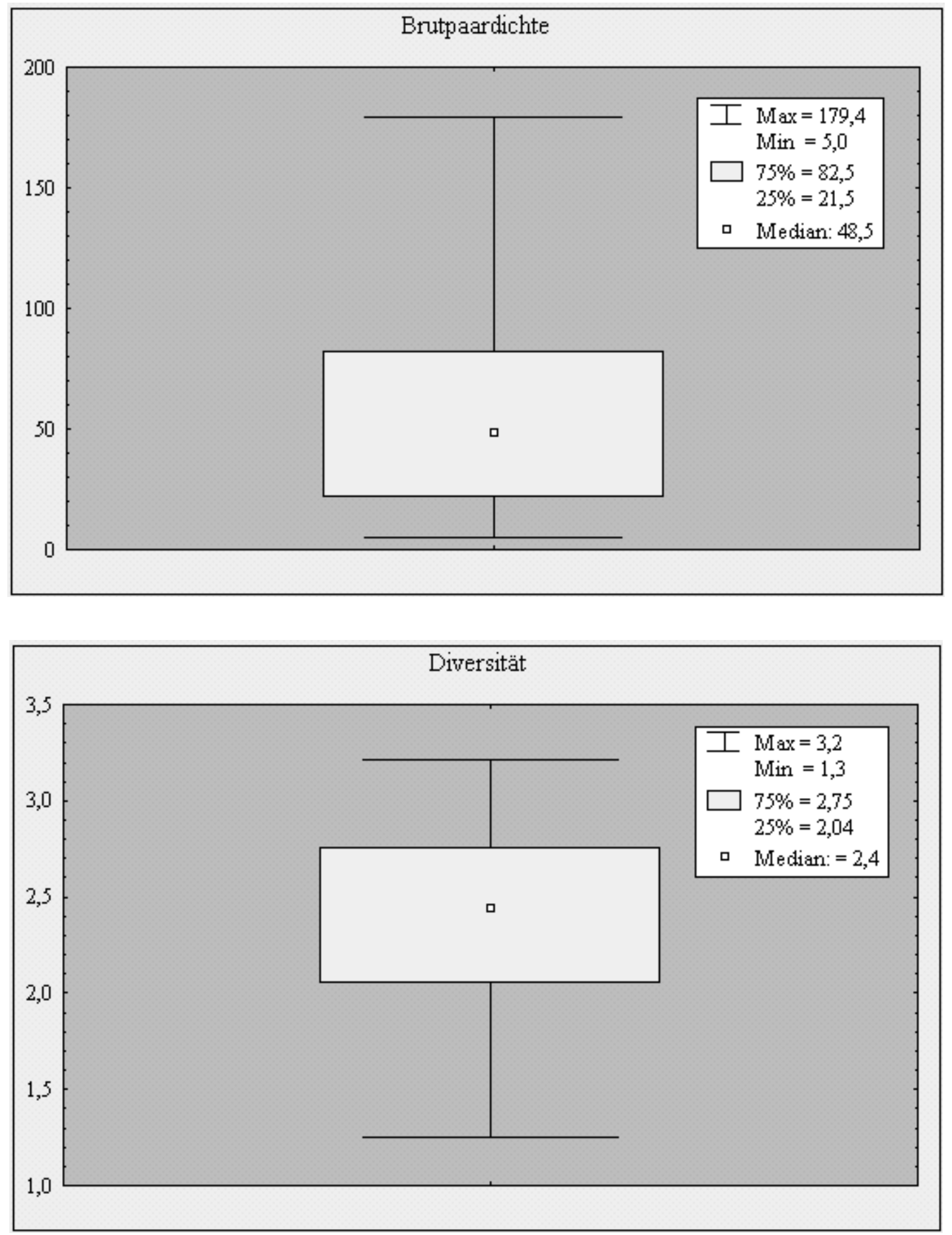

Abb. 2: Mediane der Brutpaardichte und Diversität von Vögeln in N=27 Hecken.

Die Diversität erreicht in Hecken Werte zwischen 1,3 und 3,2. Mittelwert und Median liegen bei 2,4. Es besteht kein Zusammenhang zwischen der Besiedlungsdichte und der Diversität von Vögeln in den untersuchten Hecken ( $\mathrm{rs}=0,3, \mathrm{t}=1,793$, FG 25, zweiseitig, n.s.).

D: Die Besiedlungsdichte von Vögeln in Hecken weist sehr starke Unterschiede auf. Die in dieser Studie gefundene Differenz von 5 bis zu 180 BP/km, lässt auf deutliche Unterschiede der Attraktivität von Hecken für Vögel schließen. Dieses wird ausführlich im Zusammenhang mit den Fangzahlen in Hecken unterschiedlicher Struktur dargestellt und diskutiert (Kapitel 
5.2.1, ff.). Die große Differenz der Diversitätswerte und die große Amplitude der Besiedlungsdichten, die die untersuchten Hecken aufweisen, lassen es naheliegend erscheinen, Hecken nach eben diesen Kriterien zu bewerten. Da aber Besiedlungsdichte und Diversität nicht korrelieren und es keinen objektiven Grund für die Bevorzugung eines dieser Bewertungskriterien gibt, stellt sich die Frage, wonach Hecken in ihrer Eignung für Vögel bewertet werden sollen. Da sich Habitatqualität vor allem im Reproduktionserfolg von Arten zeigt, ist es notwendig, den Bruterfolg zu erfassen. Kartierungen können das nicht leisten. Mit einem standardisierten Fangprogramm läßt sich der Bruterfolg zumindest indizieren. Zur Bewertung von Habitaten ist deshalb die Durchführung von Netzfängen der Kartierung in qualitativer Hinsicht vorzuziehen.

Hohe Diversität wird mit zunehmender Gleichverteilung der Abundanz einzelner Arten in Verbindung mit einer hohen Artenzahl erreicht. Da keine Korrelation zwischen Besiedlungsdichte und Diversität besteht, lässt sich schließen, dass Hecken mit hoher Siedlungsdichte von einem großen Anteil sehr dicht brütender Arten dominiert werden.

Die Unterschiede in der Bandbreite von Besiedlungsdichten oder Artspektren sind vielfach bezüglich ihrer Abhängigkeit von Heckenstrukturen analysiert worden (GREEN et al. 1994, PARISH et al. 1994, Hinsley et al. 1995, MACDONALD \& JOHNSON 1995, SPARKS et al. 1996, PotTS 1997). HinsLeY \& BELLAMY (2000) fassen in einer Übersichtsarbeit zusammen, dass das aus der Breite und Höhe bestimmte (Gehölz-) Volumen einer Hecke der wichtigste Faktor zur Vorhersage von hoher (Brutvogel-) Abundanz oder Diversität von Vögeln ist: Je breiter und höher eine Hecke ist, desto höher sind Artenzahl bzw. Abundanz. Eine umfassende Darstellung eigener Befunde und die Diskussion bezüglich der Zusammenhänge von Brutvogelbeständen und Heckenstrukturen findet sich in Teil II der Arbeit. 


\section{Das Fangprogramm}

Der Fang von Vögeln zur individuellen Kennzeichnung mit Ringen war der zentrale methodische Ansatz im Heckenprogramm. International wird dieses z.T. schon seit den 1980er Jahren praktiziert, z.B. im Rahmen des Constant Effort Site des BTO in UK und Irland (BAillie et al. 1986, BAillie 1990, PeACH et al. 1996, BAlmer \& PeACH 1998). Ähnliche Programme wurden mittlerweile auch in den Niederlanden (MAJOOR 1996) und in weiteren europäischen Ländern gestartet. Das Programm bot die Möglichkeit, ganzjährig und unter standardisierten Bedingungen, Vögel $\mathrm{zu}$ fangen und $\mathrm{zu}$ beringen. Verbindlich war die Durchführung der Beringung zur Brutzeit, denn es war inhaltliches Ziel des Programms, grundlegende Erkenntnisse über

1. die Brutvogelbestände in unterschiedlich strukturierten Hecken,

2. die Anteile der Jungvögel (zur Indizierung des Bruterfolges) in den Jahren und

3. die Populationsdynamik dieses Habitattyps zu gewinnen.

Das Fangprogramm verfolgte somit zwei Anliegen: Erstens sollten populationsbiologische Kennwerte von Vogelbeständen in Hecken gewonnen werden, und zweitens diente das Programm der Erprobung des standardisierten Netzfangs für ein nationales Monitoring an Kleinvogel-Populationen.

\subsection{Methode: Standardisierter Netzfang durch konstanten Aufwand in der Brutzeit}

Die Standardisierung ist wesentlicher Bestandteil der Methodik, um eine Vergleichbarkeit der gewonnenen Daten zwischen verschiedenen Standorten und über die Jahre hinweg zu ermöglichen. Nur so lassen sich qualitative und quantitative Unterschiede der Vogelbestände zwischen einzelnen Hecken und die Entwicklung der Brutvogelbestände in den jeweiligen Probeflächen erfassen, weil der Fangerfolg vom betriebenen Fangaufwand abhängt.

Der Netzfang wurde mit einer konstanten Anzahl von Netzen an gleichbleibenden Standorten mit konstantem zeitlichen Aufwand und Fangbeginn durchgeführt.

Die Netzstandorte wurden vor Beginn der Untersuchung ausgewählt und für den gesamten Zeitraum der Untersuchung festgelegt. Damit wurde auch die Anzahl der Netze und die Fangfläche bestimmt. Die Auswahl der jeweiligen Standorte, die Netzlänge an den Fangplätzen und deren Abstand zueinander wurde den Besonderheiten der Untersuchungsflächen angepasst und war den Mitarbeitern vor Ort überlassen.

Zur Konstanthaltung des Aufwandes wurde jeweils ein Fangtag von sechs Stunden Fangzeit, mit Beginn bei Sonnenaufgang (oder eine Stunde vorher), zu jeder Dekade in der Brutzeit 
festgelegt. Zwischen den einzelnen Fangtagen sollten mindestens sechs Tage Fangpause liegen, um Gewöhnungseffekte an die Netze zu vermeiden. Die Einteilung der Dekaden war vorgegeben (s. Tab. 3). Die Benutzung jeglicher Lockmittel (künstliche Tränke, Einsatz von Klangattrappen oder Lockvögeln, Futterplätze, o.ä.) war untersagt. Alle Festlegungen zur Standardisierung des Fangprogramms, wie auch der Kartierungen und der Heckenbeschreibung, wurden den Mitarbeitern durch die „Arbeitsanleitung für Heckenuntersuchungen“ der Vogelwarten bekanntgegeben.

Der Netzfang bietet die Möglichkeit der individuellen Kennzeichnung von Vögeln. Alle erstmals gefangenen Vögel werden dafür mit Aluminiumringen, auf denen eine individuelle Kennnummer und die zuständige Vogelwarte eingestanzt ist, beringt. Vögel die bereits einen Ring tragen werden als Wiederfänge bezeichnet, wenn sie im laufenden Kalenderjahr beringt worden sind. In Vorjahren oder von anderen Bearbeitern beringte Vögel werden als Kontrollfänge vermerkt. Durch Wiederfänge in der Brutperiode können standorttreue Revierinhaber von Durchzüglern oder revierlosen 'Floatern' unterschieden werden. Eine solche Trennung ist selbst bei sorgfältig durchgeführten Revierkartierungen nicht möglich. In der Hand läßt sich das Alter und häufig auch das Geschlecht von Vögeln anhand von Gefieder- und Körpermerkmalen bestimmten (SVENSSON 1992, JENNI \& WINKLER 1994). Aus dem Verhältnis von Jungvögeln zu Altvögeln läßt sich bei Standardisierung des Fangaufwandes der jährliche Bruterfolg indizieren und über die Jahre oder zwischen verschiedenen Untersuchungsgebieten vergleichen (BAIRLEIN et al. 2000). Mit der Kennzeichnung von Individuen ist darüber hinaus die Berechnung von Überlebensraten möglich, indem der Anteil der Kontrollfänge von den am Standort beringten Individuen bestimmt wird. Diese Optionen gehen über das regelmäßige und standardisiert ablaufende Erfassen von Vogelbeständen (wie unter 4.1 angeführt) hinaus. Eine wichtige Voraussetzung für den Erfolg des standardisierten Netzfangs in der Brutzeit ist jedoch eine langfristig angelegte Programmlaufzeit, was sowohl das Populationsmonitoring als auch die Erhebung von Überlebensraten anbetrifft. In der vorliegenden Arbeit liegt der Schwerpunkt auf dem Vergleich von Arten und Artendichten in verschieden strukturierten Hecken und der Indizierung des relativen Bruterfolgs über die Jahre. Die Bestandsdynamik wird dargestellt, es können aber noch keine gesicherten Bestandstrends aus den Ergebnissen abgeleitet werden.

\subsubsection{Durchführung und Durchführbarkeit des standardisierten Netzfangs}

Für die Periode der Brutzeit ist der Zeitraum vom 1. April bis 18. August in 14 Dekaden eingeteilt. Jede Dekade ist festgelegt (Tab. 3) und mit einer Nummer versehen. 
Tab. 3: Einteilung der Dekaden und numerische Zuordnung.

\begin{tabular}{|l|c|c|c|c|c|c|c|}
\hline Nummer & 1 & 2 & 3 & 4 & 5 & 6 & 7 \\
\hline Dekade & $1 .-10$. Apr & $11 .-20$. Apr & $21 .-30$. Apr & $1 .-10$. Mai & $11 .-20$. Mai & 21.-30. Mai & 31. Mai-9. Jun \\
\hline Nummer & 8 & 9 & 10 & 11 & 12 & 13 & 14 \\
\hline Dekade & $10 .-19$. Jun & 20.-29. Jun & 30. Jun-9. Jul & $10 .-19$. Jul & 20.-29. Jul & 30. Jul-8. Aug & 9.-18. Aug \\
\hline
\end{tabular}

Jeder Mitarbeiter sollte in der Brutzeit mindestens 10 Fangtermine und maximal einen Fangtag pro Dekade durchführen. In Abb. 3 ist die Anzahl der tatsächlich durchgeführten Fangtage dargestellt.

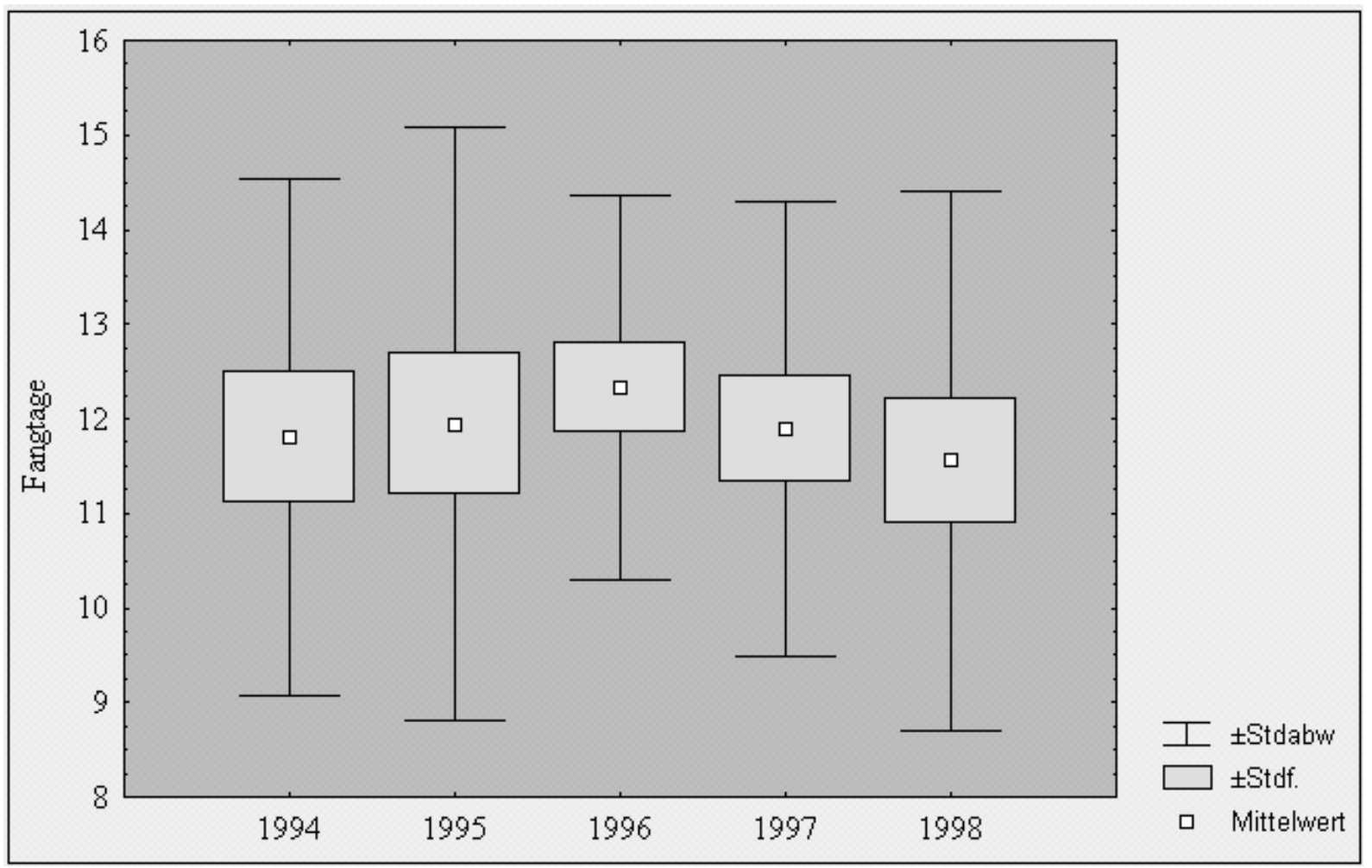

Abb. 3: Varianz der von allen Bearbeitern (n=23) durchgeführten Anzahl von Fangtagen über fünf Jahre Untersuchungszeitraum. Die Anzahl der jährlich bearbeiteten Untersuchungsgebiete ist in Tab. 4 angegeben.

Der Mittelwert pendelte um 12 Fangtage (Abb. 3). Nach relativ weiter Streuung der Fangtage in den ersten beiden Jahren wurde 1996 sehr streng nach den Vorgaben gearbeitet. In den verbleibenden zwei Jahren nahm die Aktivität wieder etwas ab.

In allen Untersuchungsjahren lag die Anzahl durchgeführter Fangtage in einzelnen Untersuchungsflächen außerhalb der Vorgaben. Dieses betrifft sowohl Überschreitungen als auch Unterschreitungen gleichermaßen. Nur vier Bearbeiter arbeiteten über die gesamte Laufzeit entsprechend der festgelegten Programmvorschriften. In $20 \%$ der jährlichen Fangperioden wurden weniger als 10 Fangtage durchgeführt, $14 \%$ wiesen dagegen zu viele Fangtage auf $(>14)$. Für die Auswertung im Sinne eines Monitorings war es deshalb notwendig, einzelne Untersuchungsflächen oder -jahre auszuschließen. Im Bestreben um eine umfassende 
ökologische Bewertung von Hecken, war es teilweise erforderlich, mit möglichst umfangreichem Datenmaterial zu arbeiten. In diesen Fällen wurden alle Fangsummen mit der Anzahl von durchgeführten Fangtagen in Beziehung gesetzt. In den Tabellen und Abbildungen sind die Anzahlen betrachteter Untersuchungsgebiete und homogenisierende Rechenschritte jeweils angegeben.

Insgesamt beteiligten sich 23 Mitarbeiter mit 33 Hecken, davon arbeiteten 14 Beringer an 21 betreuten Hecken über die Gesamtdauer des Programms. Das Ausscheiden von Mitarbeitern wurde weitgehend durch neu hinzukommende ausgeglichen, so dass die mittlere Anzahl durchgeführter Fangtage über die Jahre 1994 bis 1998 zwischen 11,6 und 12,3 lag (Tab. 4).

Tab. 4: Durchschnittliche Anzahl der jährlich durchgeführten Fangtage bei allen 23 Bearbeitern.

\begin{tabular}{|l|r|r|r|r|r|}
\hline & \multicolumn{1}{|c|}{1994} & 1995 & 1996 & 1997 & 1998 \\
\hline Summe Fangtage & 177 & 203 & 222 & 214 & 208 \\
Untersuchungsgebiete [n] & 15 & 17 & 18 & 18 & 18 \\
\hline Fangtage [Durchschnitt] & 11,8 & 11,9 & 12,3 & 11,9 & 11,6 \\
\hline
\end{tabular}

Ein Vergleich von Einzelflächen über die Jahre innerhalb des Programms ist möglich, weil die Bearbeiter über den gesamten Zeitraum hinweg mit konstantem Aufwand gearbeitet haben. Für die Darstellung des Populationsmonitoring bietet sich die Auswahl der Flächen an, die über die gesamte Laufzeit des Programms beteiligt waren.

Fazit: Die Qualität des Programms hängt von der Disziplin und der Anzahl der Bearbeiter ab. Ausgelassene Dekaden erzeugen Datenlücken und mehrfaches Fangen innerhalb einer Dekade hat nicht nur mehr Arbeit in der Auswertung zur Folge, sondern wirkt sich auch auf den Fangerfolg in Folgedekaden aus. Fast zwei Drittel der Hecken wurden über den gesamten Zeitraum bearbeitet. Das spiegelt eine hohe Verbindlichkeit der Mitarbeiter wider. Bei einem langjährig angelegten Monitoring sollten neben den jährlich stattfindenden Beringertagungen der Vogelwarten auch methodisch-praktische Kurse zur Übung für neu anfangende Beringer und zum gegenseitigen Erfahrungsaustausch unter Anwesenheit von Mitarbeitern der Vogelwarten angeboten werden. Damit könnte auf Dauer auch eine bessere Homogenisierung des Fangaufwandes erreicht werden.

\subsubsection{Auswahl der Programmvögel}

Nicht alle beringten oder als territorial erfassten Arten sind als typische und regelmäßige Heckenbrüter $\mathrm{zu}$ betrachten. Lokale Besonderheiten, Verbreitungsgrenzen von Arten, regionale Einbettung der Hecke und die geographische Nähe der Untersuchungsgebiete zu anderen Habitattypen können das Auftreten von Arten begründen, die der Hecke an sich nicht 
zuzuordnen wären. Im Anhang (Tab. 45) sind in einem Gesamtverzeichnis unter 'Habit' betreffende Arten entsprechend ihrer Habitatzuordnung oder ihres Status gekennzeichnet. Um eine bessere Vergleichsbasis $\mathrm{zu}$ den Ergebnissen aus den verschiedenen Untersuchungsflächen oder Revierkartierungen herzustellen, wird eine Auswahl von „Programmvögeln“ getroffen, die, wenn nicht anders angezeigt, im Folgenden für alle vergleichenden Betrachtungen als Bezugsbasis gilt. Vögel für die folgende Kriterien zutreffen, wurden nicht als Programmvögel aufgenommen und die Kürzel sind in der Übersicht aller beringten Vögel in Tab. 45 (im Anhang) unter 'Habit' angegeben:

- W - Wald

- O - Offenland

- G - Gewässer, Feuchtgebiete

- D - Dorf, Gebäude, u.a.

- S - Seltenheit

- $\mathrm{nSi}$ - Nichtsingvogel ausgeprägter Waldvogel, kommt ohne Waldanbindung nicht in Hecke vor, z.B. Waldlaubsänger

Bewohner der offenen Feldflur, meiden u.U. sogar Gehölzstrukturen, z.B. Feldlerche

Vögel sind nur aufgrund unmittelbarer Gewässernähe in oder an Hecken anzutreffen, z.B. Teichrohrsänger

Vorkommen begründet sich durch Bauwerke in der Hecke oder deren Nähe, z.B. Hausrotschwanz

Verbreitungsgrenze liegt im Untersuchungsareal, so dass die Art nicht in allen Untersuchungsgebieten vorkommen kann, z.B. Sperbergrasmücke

Alle Nichtsingvögel werden ausgeschlossen, weil sowohl Netzfang als auch Revierkartierung keine geeigneten Methoden zu deren standardisierter Erfassung sind, z.B. Sperber 
Die Auswahl der Programmvögel umfasst folgende 38 Arten:

Tab. 5: Die Programmvögel des Heckenprogramms der deutschen Vogelwarten, in alphabetischer Reihenfolge.

\begin{tabular}{|lll|}
\hline 1 Amsel & 14 Gimpel & 27 Rotkehlchen \\
2 Baumpieper & 15 Girlitz & 28 Schwanzmeise \\
3 Blaumeise & 16 Goldammer & 29 Singdrossel \\
4 Bluthänfling & 17 Grauschnäpper & 30 Star \\
5 Buchfink & 18 Grünling & 31 Stieglitz \\
6 Dorngrasmücke & 19 Heckenbraunelle & 32 Sumpfmeise \\
7 Feldschwirl & 20 Kernbeißer & 33 Sumpfrohrsänger \\
8 Feldsperling & 21 Klappergrasmücke & 34 Trauerschnäpper \\
9 Fitis & 22 Kleiber & 35 Wacholderdrossel \\
10 Gartenbaumläufer & 23 Kohlmeise & 36 Weidenmeise \\
11 Gartengrasmücke & 24 Mönchsgrasmücke & 37 Zaunkönig \\
12 Gartenrotschwanz & 25 Nachtigall & 38 Zilpzalp \\
13 Gelbspötter & 26 Neuntöter & \\
\hline
\end{tabular}

Die Beringungssummen der Programmvögel aller Hecken sind in ihrem Jahresdurchschnitt in Abb. 4 zusammengefasst und nach Alter der Erstberingten getrennt.

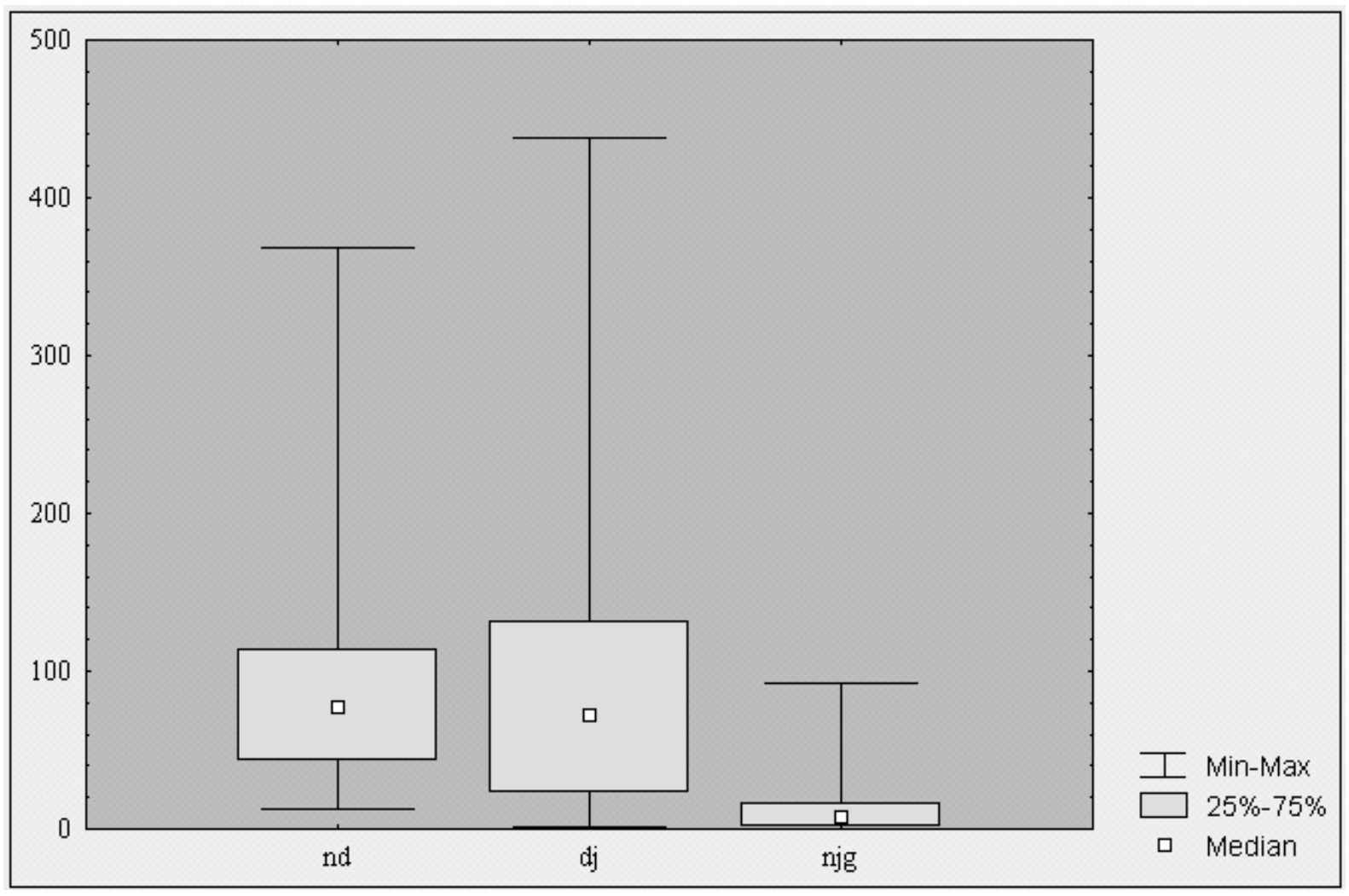

Abb. 4: Jahresberingungssummen adulter (nd), juveniler (dj) und nestjunger (njg) Programmvögel in 33 Hecken.

Im Jahresmittel wurden in einer Hecke zur Brutzeit 97 adulte Vögel beringt. Dazu kommen durchschnittlich 91 Jungvögel aus dem Netzfang und etwa 15 Beringungen an Nestlingen. Da 
sich nicht alle Mitarbeiter an der Nestersuche beteiligt haben, liegt der Durchschnitt bei über 20 Nestlingsberingungen in den 23 Hecken, in denen überhaupt Nestlinge beringt worden sind. Es gibt keinen summarischen Unterschied zwischen den Fangsummen adulter (nd=nicht diesjähriger) und juveniler ( $\mathrm{dj}=$ diesjähriger) Vögel. Einige Hecken wiesen sehr hohe Beringungszahlen auf (über 300 Alt- oder über 400 Jungvögel in der Jahressumme), in anderen Hecken wurden dagegen fast keine Jungvögel beringt (Abb. 4).

\subsection{Arten und Abundanzen}

E: Insgesamt wurden zur Brutzeit 30.817 Vögel von 89 Arten als Erstfänge (EF) beringt. Davon sind 14.729 adult (oder nicht eindeutig einer Altersklasse zuzuordnen; nd/F= nicht diesjährig/Fängling), 13.999 als im Untersuchungsjahr geboren (dj=diesjährig) und 2.089 als Nestlinge (njg=nestjung) beringt worden (Übersicht in Tab. 45).

Von 55 Arten wurden 10 oder mehr Individuen als Adulte in der Brutzeit beringt. Von 67 Arten wurden Jungvögel gefangen und von 28 Arten Nestlinge beringt. 67 Arten (=76\%) erreichten weniger als $1 \%$ von allen adult beringten Individuen (subrezedente Arten). Mit der Konzentration auf 38 Programmvogelarten (s. 5.2.2) wird erstens eine gestraffte Datenbasis auf der Ebene der Arten hergestellt und es werden zweitens immer noch über $90 \%$ aller Erstfänge berücksichtigt. Alle folgenden Darstellungen von Abundanzverhältnissen beziehen sich auf die Angaben in Tab. 6.

Die häufigsten Arten sind Mönchsgrasmücke und Zilpzalp, die zusammen ein Viertel aller Erstfänge ausmachen (26,5\%). Über $93 \%$ entfallen auf die häufigsten 20 Arten (nd=93,1\%, $\mathrm{dj}=94,2 \%)$. Nach den als eudominant ( $>10 \%$ der Individuen) $\mathrm{zu}$ bezeichnenden Arten Mönchsgrasmücke und Zilpzalp folgen (in absteigender Reihenfolge) mit Gartengrasmücke, Grünling, Rotkehlchen, Amsel, Sumpfrohrsänger und Heckenbraunelle weitere Arten, die mehr als $5 \%$ der Individuen stellen (dominante Arten).

Von 28 Arten konnten Nestjunge beringt werden. Darunter sind 11 Höhlen- und Halbhöhlenbrüter, auf welche über $70 \%$ der Beringungen an Nestlingen entfallen. Allein die Beringungen an jungen Kohl- und Blaumeisen machen über die Hälfte aller Nestlingsberingungen aus. 
Tab. 6: Übersicht der Beringungszahlen von adulten und juvenilen Erstfängen und Nestlingen der Programmvögel sowie Dominanz [\%] jeder Art für das betreffende Alter.

\begin{tabular}{|c|c|c|c|c|c|c|c|}
\hline & Art & EF nd/F & $\%$ nd $/ F$ & EF dj & $\% \mathbf{d j}$ & njg & $\%$ njg \\
\hline 1 & Mönchsgrasmücke & 1767 & 13,34 & 1670 & 12,86 & 64 & 3,1 \\
\hline 2 & Zilpzalp & 1487 & 11,23 & 2028 & 15,62 & 52 & 2,5 \\
\hline 3 & Gartengrasmücke & 802 & 6,05 & 650 & 5,01 & 24 & 1,2 \\
\hline 4 & Grünling & 779 & 5,88 & 299 & 2,30 & 49 & 2,4 \\
\hline 5 & Rotkehlchen & 773 & 5,84 & 611 & 4,70 & 30 & 1,5 \\
\hline 6 & Amsel & 751 & 5,67 & 584 & 4,50 & 104 & 5,1 \\
\hline 7 & Sumpfrohrsänger & 722 & 5,45 & 211 & 1,62 & 6 & 0,3 \\
\hline 8 & Heckenbraunelle & 665 & 5,02 & 464 & 3,57 & 56 & 2,7 \\
\hline 9 & Kohlmeise & 586 & 4,42 & 1448 & 11,15 & 647 & 31,4 \\
\hline 10 & Dorngrasmücke & 578 & 4,36 & 471 & 3,63 & 25 & 1,2 \\
\hline 11 & Fitis & 577 & 4,36 & 475 & 3,66 & 7 & 0,3 \\
\hline 12 & Goldammer & 496 & 3,74 & 303 & 2,33 & 15 & 0,7 \\
\hline 13 & Buchfink & 417 & 3,15 & 116 & 0,89 & 22 & 1,1 \\
\hline 14 & Star & 402 & 3,03 & 1029 & 7,92 & 129 & 6,3 \\
\hline 15 & Feldsperling & 310 & 2,34 & 472 & 3,63 & 244 & 11,9 \\
\hline 16 & Klappergrasmücke & 309 & 2,33 & 116 & 0,89 & 22 & 1,1 \\
\hline 17 & Singdrossel & 284 & 2,14 & 284 & 2,19 & 52 & 2,5 \\
\hline 18 & Blaumeise & 244 & 1,84 & 839 & 6,46 & 429 & 20,8 \\
\hline 19 & Nachtigall & 194 & 1,46 & 114 & 0,88 & & \\
\hline 20 & Gelbspötter & 191 & 1,44 & 55 & 0,42 & & \\
\hline 21 & Stieglitz & 120 & 0,91 & 58 & 0,45 & & \\
\hline 22 & Zaunkönig & 113 & 0,85 & 156 & 1,20 & 20 & 1,0 \\
\hline 23 & Neuntöter & 108 & 0,82 & 78 & 0,60 & 19 & 0,9 \\
\hline 24 & Weidenmeise & 79 & 0,60 & 91 & 0,70 & 2 & 0,1 \\
\hline 25 & Gimpel & 70 & 0,53 & 32 & 0,25 & & \\
\hline 26 & Gartenrotschwanz & 67 & 0,51 & 28 & 0,22 & 5 & 0,2 \\
\hline 27 & Kernbeißer & 51 & 0,38 & 11 & 0,08 & & \\
\hline 28 & Schwanzmeise & 46 & 0,35 & 46 & 0,35 & & \\
\hline 29 & Girlitz & 44 & 0,33 & 28 & 0,22 & & \\
\hline 30 & Trauerschnäpper & 40 & 0,30 & 16 & 0,12 & 16 & 0,8 \\
\hline$\overline{31}$ & Sumpfmeise & 34 & 0,26 & 78 & 0,60 & & \\
\hline 32 & Grauschnäpper & 29 & 0,22 & 18 & 0,14 & & \\
\hline 33 & Feldschwirl & 24 & 0,18 & 5 & 0,04 & & \\
\hline 34 & Wacholderdrossel & 23 & 0,17 & 21 & 0,16 & & \\
\hline 35 & Bluthänfling & 23 & 0,17 & 5 & 0,04 & 12 & 0,6 \\
\hline 36 & Gartenbaumläufer & 17 & 0,13 & 35 & 0,27 & & \\
\hline 37 & Kleiber & 15 & 0,11 & 39 & 0,30 & 7 & 0,3 \\
\hline 38 & Baumpieper & 10 & 0,08 & 3 & 0,02 & & \\
\hline & Summe & 13247 & & 12987 & & 2058 & \\
\hline
\end{tabular}

D: Im Fangprogramm können über alle 74 nachgewiesenen Singvogelarten 25,7 \% Höhlenbrütern zugerechnet werden. Das entspricht etwa dem Anteil von $28 \%$ der in Deutschland brütenden Höhlenbewohner (nach Auswertung der Angaben in BEZZEL 1993 für N=104 betrachtete Singvogelarten).

Bei den Jungvögeln weichen die Häufigkeitsverhältnisse etwas von denen der Altvögel ab. Es treten, neben den gleichbleibend dominierenden Arten Zilpzalp (15,6 \%) und Mönchsgrasmücke $(12,9 \%)$, mit Kohlmeise $(11,1 \%)$, Star (7,9\%) und Blaumeise $(6,5 \%)$ vor allem 
Höhlenbrüter in höherer Abundanz in den Hecken auf. Dieses ist unter Umständen weniger auf das Habitat selbst, als vielmehr auf die hohe Dichte von künstlichen Nisthöhlen zurückzuführen, die in vielen der bearbeiteten Hecken oder in deren unmittelbarer Umgebung angebracht sind. Wenn ausreichend Nisthöhlen zur Verfügung stehen, sind Höhlenbrüter, aufgrund ihrer hohen Anzahl von Nestlingen und geringerer Verluste durch Prädation (MARTIN \& PINGJUN 1992, MARTIN 1993b, BAIRLEIN 1996a), gegenüber frei brütenden Arten bezüglich des Ausfliegeerfolgs im Vorteil.

Bemerkenswert ist die Differenz der Fanghäufigkeit von Jung- und Altvögeln bei Grünling, Teich- und Sumpfrohrsänger. Durch den deutlichen Abfall der Fänge wird bestätigt, dass die hohen Anzahlen adulter Individuen der beiden ersten Arten nicht auf die Höhe der Brutbestände in den Hecken zurückgeführt werden kann. Auch Sumpfrohrsänger nutzen vielfach an die Hecke angrenzende Strukturen, wie z.B. breite Brennesselfluren oder Rapsfelder (früher auch verunkrautete Getreidefelder), um darin zu brüten (GLUTZ VON BLOTZHEIM \& BAUER 1991a, BEZzEL 1993). Es ist anzunehmen, dass aus diesem Grund weniger Jungvögel in den Hecken gefangen werden als dies nach der Häufigkeit der Altvögel dieser Art anzunehmen wäre.

Die Habitatansprüche der Arten sind sehr heterogen. Neben typischen Vertretern der Waldavifauna treten Bewohner halboffener Landschaften auf. Unter dem Begriff der 'halboffenen Landschaft' seien hier Landschaftstypen verstanden, die durch das Fehlen ausgedehnter und zusammenhängender Waldgebiete gekennzeichnet sind, wobei einzelne Gehölzstrukturen als Einzelbaum, Hecke, gebüschbestandene Brache, Feldgehölzinsel, etc. vorhanden sind (Blana 1978, BEZzel 1982, Flade 1994, EllenberG 1996, Ringler et al. 1997, s.a. Glossar).

Unter den häufigsten 10 Arten dominieren solche, die ursprünglich als Waldvögel, mittlerweile auch als Besiedler von Gärten, Parks, Friedhofsanlagen usw. in die Nähe menschlicher Siedlungsbereiche vorgedrungen sind. Die meisten Arten, die regelmäßig in Hecken vorkommen, zeichnen sich durch gute Anpassungsfähigkeit aus und sind, als Arten mit großer ökologischer Bandbreite, auch in anderen Lebensräumen häufig. Dieses gilt gleichermaßen für Invertebraten. Nach Untersuchungen von TISCHLER (1948b) können etwa 50-95 \% der in Hecken vorkommenden Laufkäferarten als Waldarten bezeichnet werden. Weitere Arten lassen sich als Ubiquisten oder eurytope Arten des Offenlandes bezeichnen (BLAB 1993).

Die als 'typische Heckenbewohner' bezeichneten Neuntöter, Goldammer und Dorngrasmücke (FLADE 1994, Ringler et al. 1997) sind nicht so häufig wie einige eurytope Arten. Goldammer und Dorngrasmücke erreichen in allen Hecken hohe Stetigkeiten, wobei der Neuntöter 
nur in wenigen der untersuchten Hecken vorkommt. Das Spektrum der in dieser Studie untersuchten Hecken ist relativ groß und entspricht nicht immer den eng gefassten Habitatansprüchen dieser Arten (JAKOBER \& StAuber 1981, Glutz VON BlotzheIM \& BAUER 1991b, Lille 1996, ScotT Morales 1997), die auch auf die Nutzung der umgebenden Landschaft angewiesen sind. Beispielsweise bieten Gartenhecken, üblicherweise in urbanem Umfeld gelegen, auch bei höchstem Struktur- und Pflanzenartenreichtum keinen geeigneten Lebensraum für Neuntöter, Goldammer oder Dorngrasmücke.

\subsubsection{Gibt es bevorzugte Heckenstrukturen?}

Vögel können Hecken nur dann nutzen, wenn die Heckenstrukturen den Habitatansprüchen der betreffenden Vogelart entsprechen. Insbesondere in der Brutzeit haben Hecken als Neststandort, Ort der Nahrungssuche, als Schutzraum vor Feinden, als Singwarte, zur Nestbewachung, als Jagdansitzwarte oder als Schlafplatz vielfältige Funktionen zu erfüllen. Die Entscheidung über die Eignung einer Habitatstruktur erfolgt über das Individuum. Dennoch lassen sich artspezifische Präferenzen zuordnen, die mit dem Vorhandensein bestimmter Strukturen oder Ressourcen verbunden sind. Anhand von tierökologischen Kennwerten wie z.B. Bestandsdichten, Artenzahlen oder Reproduktionserfolgen sind dann auch artübergreifend Rückschlüsse auf die Attraktivität oder den Wert eines Lebensraumes möglich. Dieses soll in diesem Kapitel zunächst auf der Ebene von den in der Hecke auftretenden Individuenzahlen dargestellt werden. In einem ersten Schritt wird getestet, ob sich die Fangsummen entsprechend der Ausprägung von Strukturmerkmalen in den Hecken unterscheiden. Zweitens wird die Nutzung der Hecken auf der Ebene von Arten, anhand der unterschiedlichen Dominanzverhältnisse in den jeweiligen Heckenstrukturen, verglichen. Drittens folgt eine Einteilung von Vogelarten, deren aufgrund von Fanghäufigkeiten gefundenen Habitatpräferenzen in verschiedenen Heckentypen eine Zuordnung zu waldartigen oder halboffenen Strukturen zulassen.

M: Die Aufnahme der Heckenstrukturen war durch den Heckenerfassungsbogen standardisiert und läßt eine Einteilung der Hecken nach folgenden Strukturmerkmalen zu:

- Alter: Hecken bis 20 Jahre und ältere

- Gehölzdichte: Hecken mit einzelnen und großen Lücken und Hecken mit geschlossenem Gehölzbestand

- Breite: Hecken bis $6 \mathrm{~m}$ Breite und solche mit $>6 \mathrm{~m}$ durchschnittlicher Breite

- Höhe: Hecken ab 8 m maximaler Höhe gegen Hecken geringerer Höhe

Für jede Hecke wurde die durchschnittliche Anzahl gefangener adulter Individuen auf 100 Meter Heckenlänge, zwischen dem 1. April und dem 18. August, über die Jahre gemittelt. Die 
so erhaltenen Mittelwerte der Fangsummen wurden entsprechend der Strukturen zugeordnet und dann jeweils durch die Anzahl der Hecken geteilt. Über den Vergleich der Mittelwerte lässt sich ersehen, ob die längenbereinigten Fangsummen der Programmvögel zwischen verschiedenen Strukturen Unterschiede aufweisen. Der Fangerfolg ist das Äquivalent für den Individuenreichtum eines Habitats.

E: In neun alten Hecken ( $>20$ Jahre) wurden signifikant mehr Vögel gefangen als in 19 jüngeren ( $\mathrm{t}$-Test mit FG 26, $\mathrm{t}=2,622 ; \mathrm{p}=0,014)$. Ein Unterschied zwischen breiten $(>6 \mathrm{~m})$ und schmaleren Hecken besteht nicht ( $\mathrm{p}=0,18$; n.s., $\mathrm{t}=1,38)$. Die graphische Darstellung der Mittelwerte lässt jedoch vermuten, dass mit einer größeren Stichprobe unterschiedliche Fangzahlen zwischen breiten und schmaleren Hecken gefunden werden könnten. Die Höhe und die Lückigkeit der Hecke zeigen keinen Einfluss auf die Fangzahlen (Abb. 5).

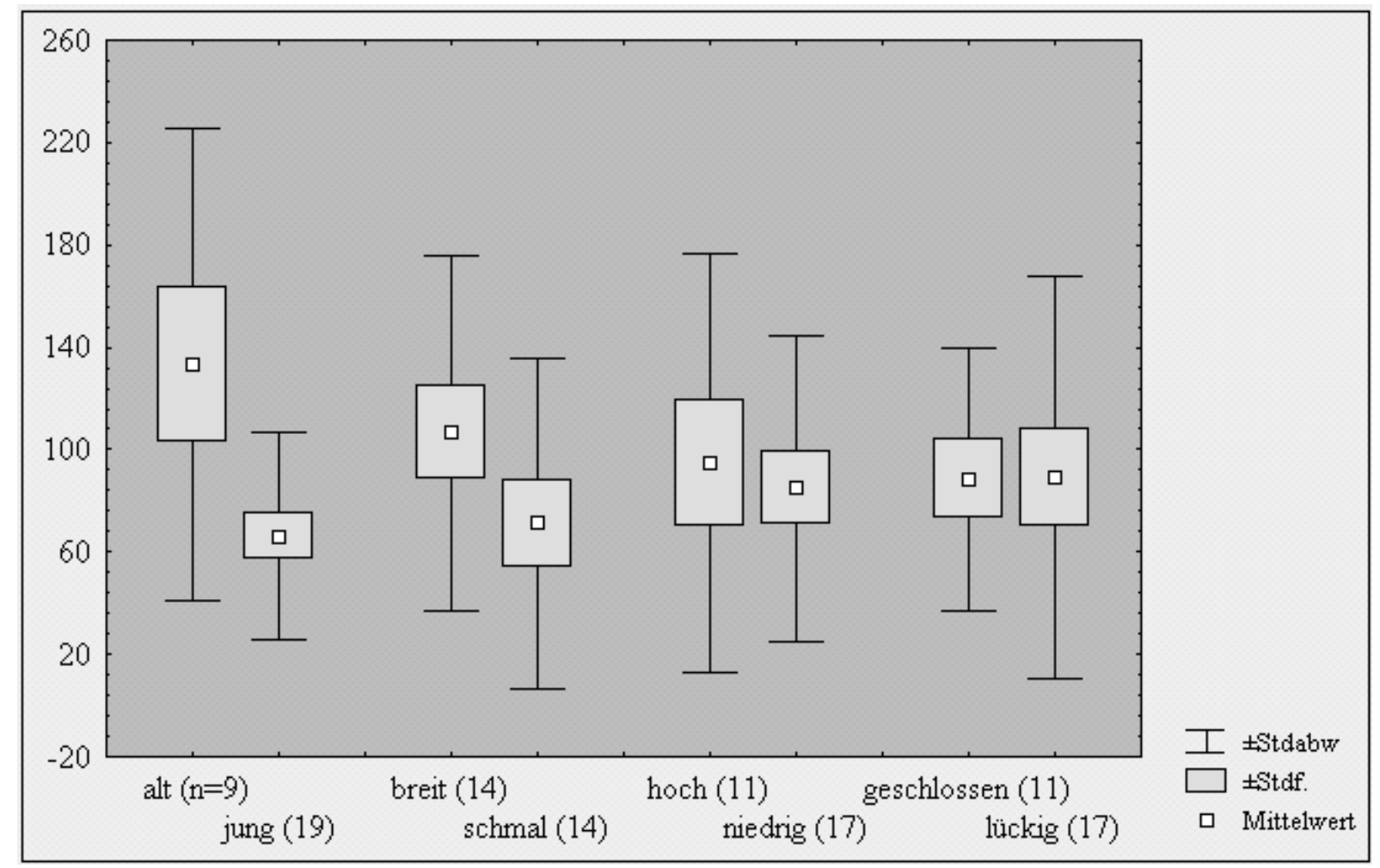

Abb. 5: Darstellung durchschnittlicher jährlicher Fangsummen aller Programmvogelarten in Hecken nach Strukturmerkmalen. Angegeben sind die Mittelwerte mit Standardabweichung und Standardfehler der gemittelten Fangsummen pro Hecke und Jahr für Alter*, Breite, Höhe und Lückigkeit.

Von den vier betrachteten Parametern ist das Alter einer Hecke der bedeutendste Faktor für den Fangerfolg. Das Alter ist somit ein wichtiges Qualitätsmerkmal einer Hecke. Während Breite und Gehölzdichte und mit Einschränkung auch die Höhe einer Hecke bei der Anlage oder durch Management-Maßnahmen beeinflusst werden können, ist das Alter nur durch den langfristigen Erhalt von Hecken zu erreichen. Dieses wiederum ist nur durch regelmäßige 
Pflege oder Nutzung möglich, weil aufwachsende Bäume durch Schattenwurf die Gebüschstruktur von Hecken sonst im Laufe der Zeit zerstören.

Bei dem Ersatz von alten Hecken durch jüngere Strukturen ist unmittelbar mit einem Individuen- und Artenverlust von Vögeln zu rechnen (Abb. 5). Dieses spiegelt sich auch in botanischen Untersuchungen an Hecken aus England wider. HOOPER (1994) fand bereits in den 1960er Jahren einen Zusammenhang zwischen dem Alter von Hecken und der Diversität von Baum- und Straucharten. Er formulierte die Faustregel, dass auf 100 Jahre Alter jeweils eine weitere Gehölzart in einer Hecke zu erwarten sei. Diese 'Regel' kann zwar ausschließlich in Bezug auf historische Heckenstandorte angewandt werden, zeigt aber die ökologische Bedeutung von zeitlicher Kontinuität in Lebensräumen.

In Bezug auf landschaftsgestaltende Eingriffe ist das Ziel der Schaffung von 'Lebensraumqualität' unmittelbar nicht erreichbar, wenn ökologisch wertvolle Lebensräume vor allem durch hohes Alter charakterisiert sind, denn 'alte Biotope' lassen sich nicht ad hoc im Zuge von Ausgleichs- und Ersatzmaßnahmen erschaffen. Insofern ist bei der Abwägung über die Notwendigkeit von Eingriffen, das Alter von Lebensräumen, aufgrund seiner Bedeutung für den Individuenreichtum von Vögeln zur Brutzeit, als unersetzbarer Biotopwert zu beachten.

\subsubsection{Dominanz einzelner Arten in unterschiedlichen Heckentypen}

Vögel stellen artspezifisch unterschiedliche Ansprüche an ihren Lebensraum. Deshalb ist es sinnvoll, wenn Fangergebnisse auf der Ebene von Arten verglichen werden, um Beziehungen zu verschiedenen Strukturmerkmalen von Hecken zu finden.

M: Die Abundanz der Erstfänge einzelner Arten wird als relativer Anteil am Gesamtfang (Dominanz) in den Probeflächen verglichen. Die Dominanz einer Art $D_{\text {spec }}$ wird somit berechnet als $D_{\text {spec }}=A_{n} * 100 / G_{i}$, wobei $A_{n}$ die Abundanz der jeweiligen Art und $G_{i}$ die Gesamtzahl gefangener Individuen darstellt.

Exemplarisch ist Tab. 7 mit der Dominanz der Programmvögel in den Hecken eines Alters bis bzw. über 20 Jahre (zum Zeitpunkt des Untersuchungsbeginns) dargestellt. Die Einteilung erfolgte nach ökologischen Gesichtspunkten und richtete sich auch nach dem Median der Meßwerte, um zwei vergleichbare Gruppen zu erhalten. Weitere Tabellen mit der Dominanz der Arten zu Heckendichte, Breite und Höhe finden sich im Anhang unter Tab. 46 bis Tab. 48.

E: Von fünf Arten ist die relative Häufigkeit in alten Hecken höher, acht Arten kommen signifikant häufiger in jüngeren Hecken vor (Z-Test, Tab. 7). Da Dominanz ein relatives Maß 
ist, darf nicht der Schluss gezogen werden, dass junge Hecken für ein größeres Artenspektrum von Bedeutung sind, als ältere Hecken. Das Verhältnis von fünf zu acht Arten resultiert aus der Tatsache, dass die vier häufigsten Arten in den alten Hecken dominant sind.

Die Arten sind entsprechend ihrer Häufigkeit in der ersten Strukturgruppe sortiert. In der letzten Spalte 'Rang' sind die Rangzahlen vergeben, die sich aufgrund der Dominanz der betreffenden Art in den jüngeren Heckenstrukturen ergeben. Bei großen Differenzen dieser Rangzahlen ist zumeist auch der Dominanzunterschied der Art in den betrachteten Strukturtypen signifikant (z.B. Gartengrasmücke, Rotkehlchen oder Dorngrasmücke, Grünling und Star in Tab. 7).

Die Mönchsgrasmücke ist in beiden Strukturgruppen die häufigste Art, erreicht aber in alten Hecken eine signifikant höhere Dominanz (15,5 zu 9,8). Ganz ähnlich verhält es sich beim Zilpzalp, wogegen Gartengrasmücke, Rotkehlchen und Klappergrasmücke in jüngeren Hecken auch gegenüber anderen Arten deutlich seltener sind.

Dorngrasmücke, Grünling und Star sind in Hecken bis 20 Jahre unter den fünf häufigsten Arten vertreten. Auch Zaunkönig, Gimpel, Nachtigall, Girlitz und Bluthänfling bevorzugen jüngere Hecken (Tab. 7).

Bezüglich unterschiedlicher Heckenhöhe gab es mit Mönchsgrasmücke, Zilpzalp, Rotkehlchen, Gartengrasmücke, Amsel, Buchfink und Trauerschnäpper sieben Arten, die Hecken über $8 \mathrm{~m}$ bevorzugten und ebenfalls sieben Arten, die häufiger in niedrigeren Hecken waren: Sumpfrohrsänger, Dorngrasmücke, Grünling, Gelbspötter, Star, Nachtigall und Girlitz (Tab. 48, im Anhang).

In Hecken mit lückigem Gehölzbestand hatten Gartengrasmücke, Dorngrasmücke, Sumpfrohrsänger, Feldsperling, Gelbspötter und Neuntöter höhere Dominanz, Grünling, Rotkehlchen, Klappergrasmücke, Blaumeise und Schwanzmeise dagegen in geschlossenen (Tab. 46, im Anhang).

In breiten Hecken wurden Mönchsgrasmücke, Star, Nachtigall, Gimpel und Trauerschnäpper signifikant häufiger gefangen, in schmaleren Hecken mit Buchfink, Grünling und Neuntöter nur drei Arten (Tab. 47, im Anhang). 
Tab. 7: Dominanz der Erstfänge adulter Vögel in Hecken über (n=9) bzw. bis 20 Jahre Alter (n=19); Rang bezieht sich auf die Dominanz der Arten in Hecken bis 20 Jahre.

\begin{tabular}{|c|c|c|c|c|c|c|}
\hline & \multirow[b]{2}{*}{ ART } & \multicolumn{2}{|c|}{ Heckenalter über 20 Jahre } & \multicolumn{2}{|c|}{ Heckenalter bis 20 Jahre } & \multirow[b]{2}{*}{ Rang } \\
\hline & & $\mathrm{nd} / \mathrm{F}$ & Dominanz & $\mathrm{nd} / \mathrm{F}$ & Dominanz & \\
\hline 1 & Mönchsgrasmücke & 817 & $15,50 *$ & 550 & 9,79 & 1 \\
\hline 2 & Zilpzalp & 755 & $14,32 *$ & 517 & 9,2 & 3 \\
\hline 3 & Gartengrasmücke & 415 & $7,87 *$ & 255 & 4,54 & 10 \\
\hline 4 & Rotkehlchen & 400 & $7,59 *$ & 247 & 4,4 & 11 \\
\hline 5 & Amsel & 338 & 6,41 & 301 & 5,36 & 6 \\
\hline 6 & Heckenbraunelle & 285 & 5,41 & 290 & 5,16 & 9 \\
\hline 7 & Kohlmeise & 236 & 4,48 & 294 & 5,23 & 7 \\
\hline 8 & Goldammer & 225 & 4,27 & 202 & 3,6 & 14 \\
\hline 9 & Fitis & 224 & 4,25 & 293 & 5,22 & 8 \\
\hline 10 & Klappergrasmücke & 191 & $3,62 *$ & 109 & 1,94 & 15 \\
\hline 11 & Sumpfrohrsänger & 184 & 3,49 & 237 & 4,22 & 12 \\
\hline 12 & Buchfink & 184 & 3,49 & 206 & 3,67 & 13 \\
\hline 13 & Dorngrasmücke & 183 & 3,47 & 320 & $5,70 *$ & 5 \\
\hline 14 & Singdrossel & 125 & 2,37 & 108 & 1,92 & 16 \\
\hline 15 & Blaumeise & 111 & 2,11 & 99 & 1,76 & 17 \\
\hline 16 & Feldsperling & 103 & 1,95 & 92 & 1,64 & 20 \\
\hline 17 & Grünling & 88 & 1,67 & 521 & $9,27 *$ & 2 \\
\hline 18 & Gelbspötter & 82 & 1,55 & 95 & 1,69 & 19 \\
\hline 19 & Neuntöter & 51 & 0,97 & 38 & 0,68 & 24 \\
\hline 20 & Weidenmeise & 30 & 0,57 & 43 & 0,77 & 23 \\
\hline 21 & Schwanzmeise & 28 & 0,53 & 14 & 0,25 & 30 \\
\hline 22 & Zaunkönig & 25 & 0,47 & 71 & $1,26^{*}$ & 21 \\
\hline 23 & Gartenrotschwanz & 25 & 0,47 & 29 & 0,52 & 26 \\
\hline 24 & Stieglitz & 24 & 0,46 & 15 & 0,27 & 29 \\
\hline 25 & Kernbeißer & 22 & 0,42 & 27 & 0,48 & 27 \\
\hline 26 & Trauerschnäpper & 19 & 0,36 & 17 & 0,3 & 28 \\
\hline 27 & Sumpfmeise & 17 & 0,32 & 13 & 0,23 & 33 \\
\hline 28 & Star & 17 & 0,32 & 371 & $6,61 *$ & 4 \\
\hline 29 & Gimpel & 13 & 0,25 & 57 & $1,02 *$ & 22 \\
\hline 30 & Grauschnäpper & 11 & 0,21 & 14 & 0,25 & 32 \\
\hline 31 & Nachtigall & 10 & 0,19 & 96 & $1,71^{*}$ & 18 \\
\hline 32 & Feldschwirl & 8 & 0,15 & 0 & 0 & 38 \\
\hline 33 & Wacholderdrossel & 8 & 0,15 & 11 & 0,2 & 34 \\
\hline 34 & Gartenbaumläufer & 6 & 0,11 & 10 & 0,18 & 35 \\
\hline 35 & Baumpieper & 6 & 0,11 & 3 & 0,05 & 37 \\
\hline 36 & Kleiber & 3 & 0,06 & 8 & 0,14 & 36 \\
\hline 37 & Girlitz & 2 & 0,04 & 31 & $0,55^{*}$ & 25 \\
\hline 38 & Bluthänfling & 0 & 0 & 14 & $0,25 *$ & 31 \\
\hline & Summe & 5271 & & 5618 & & \\
\hline
\end{tabular}

Alle vier Grasmücken-Arten sind in Hecken regelmäßig anzutreffen. Aufgrund sich überschneidender ökologischer Ansprüche ist die Untersuchung der Frage nach artspezifischen Habitatpräferenzen dieser Arten in Hecken interessant. 
Eine Analyse der Habitatwahl bezüglich des Alters von Hecken zeigt, dass Dorngrasmücken zur Brutzeit junge Hecken (bis 20 Jahre alt), alle anderen Grasmücken-Arten dagegen die Hecken mit über 20 Jahren bevorzugen (Abb. 6).

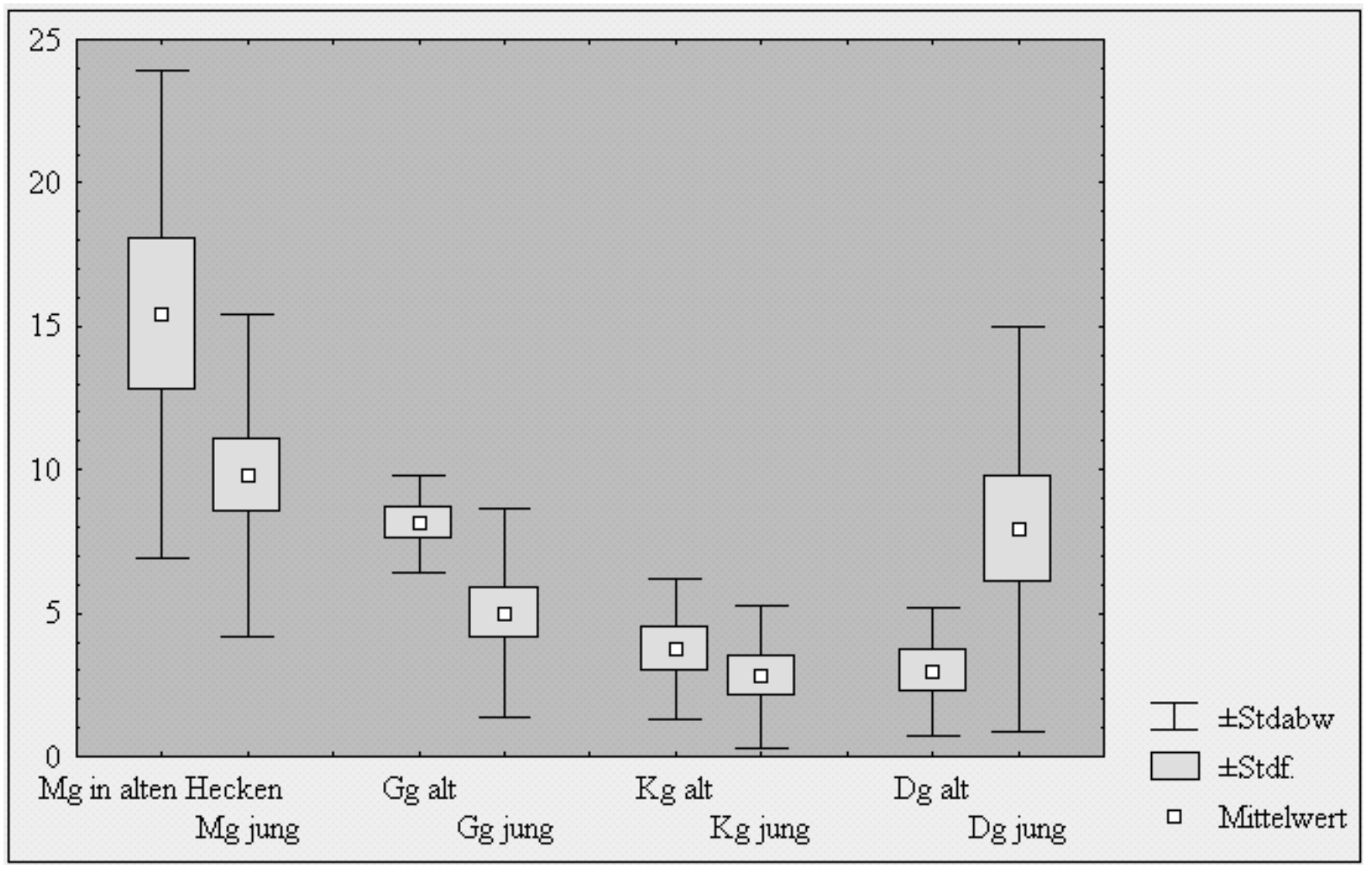

Abb. 6: Darstellung unterschiedlicher Dominanzen von vier Grasmücken-Arten in Hecken verschiedenen Alters. $\mathrm{Mg}=$ Mönchsgrasmücke, $\mathrm{Gg}=$ Gartengrasmücke, $\mathrm{Kg}=$ Klappergrasmücke, Dg=Dorngrasmücke; für alle Arten ist der Unterschied zwischen jungen und alten Hecken signifikant, Z-Test, N=28, p<0,0001.

Mönchsgrasmücke und Gartengrasmücke bevorzugen neben alten (s. Abb. 6 und Tab. 7) auch hohe Hecken (Tab. 48, im Anhang, hier erreichen beide Arten sehr hohe Dominanzwerte), sowie breite (Mg, s. Anh. Tab. 47) bzw. lückige Hecken (Gg, Anh. Tab. 46). Die Klappergrasmücke bevorzugt alte und geschlossene Hecken. Höhe und Breite einer Hecke haben für die Habitatwahl der Klappergrasmücke keinen nachweisbaren Einfluss. Die Dorngrasmücke bevorzugt deutlich jüngere Hecken und erreicht im Gegensatz zu Mönchs- und Gartengrasmücke in niedrigeren Hecken (weniger als $8 \mathrm{~m}$ maximaler Höhe) ihre höchsten Dominanzwerte (Tab. 48).

Rotkehlchen und Nachtigall bevorzugen alte Hecken, die geschlossen bzw. breit sind. Bezüglich der Höhe haben die Arten unterschiedliche Ansprüche: Die Nachtigall erreicht in niedrigeren Hecken eine höhere Dominanz als in hohen Hecken, beim Rotkehlchen ist es umgekehrt (Tab. 8). 
Aufgrund des Alters bevorzugen weder Sumpfrohrsänger noch Neuntöter eine Hecke. Beide Arten bevorzugen Hecken mit Lücken, Neuntöter zudem schmale, der Sumpfrohrsänger niedrige Hecken.

Tab. 8: Unterschiede in der Bevorzugung von 4 Heckenparametern durch ausgewählte Vogelarten, je Z-Test mit $\mathrm{N}=28$ und p<0,01 (vgl. Ergebnisse in Tab. 7, Tab. 46, Tab. 47 und Tab. 48).

\begin{tabular}{l|l|l|l|l|} 
& Nachtigall & Rotkehlchen & Neuntöter & Sumpfrohrsänger \\
\hline Alter & * bis 20 Jahre & * über 20 Jahre & n.s. & n.s. \\
Gehölzdichte & n.s. & * geschlossen & $*$ mit Lücken & * mit Lücken \\
Breite & $*$ über $6 \mathrm{~m}$ & n.s. & $*$ unter $6 \mathrm{~m}$ & n.s. \\
Höhe & $*$ niedriger $8 \mathrm{~m}$ & $*$ höher $8 \mathrm{~m}$ & n.s. & * niedriger $8 \mathrm{~m}$ \\
\hline
\end{tabular}

D: Neuntöter und Sumpfrohrsänger kommt die Unterbrechung des Gehölzbewuchses auf unterschiedliche Weise zu Gute. Für den Sumpfrohrsänger sind die (Gehölz-) Lücken von Bedeutung, wenn damit der Bestand an Brennesselfluren (Vertikalstrukturen) als Bruthabitat an der Hecke zunimmt (vgl. z.B. BEzzel 1993). Für den Neuntöter spielen frei stehende Büsche oder Heckenlücken eine wichtige Rolle bei der Ansitzjagd auf Bodenbewohner (JAKOBER \& STAUBER 1981). Beide Arten stellen deutlich unterschiedliche Ansprüche an die Beschaffenheit der gehölzfreien Lücken in den Hecken.

Die scheinbar deutliche Bevorzugung bestimmter Heckenstrukturen bei Grünling und Star (Tab. 46, Tab. 47 und Tab. 48) gehen jeweils auf hohe Fangsummen dieser Arten in einzelnen Hecken zurück und treten dort auch nur in einzelnen Jahren auf (Grünling 1994, Star 1997 und 1998). Hier sollten keine allgemeinen Schlüsse bezüglich der Habitatwahl dieser Arten gezogen werden.

Bei der Betrachtung der Dominanz so nahe verwandter Arten wie der vier Grasmückenarten und auch für die anderen gezeigten Beispiele wird deutlich, dass jede Art nur ein bestimmtes Spektrum von Heckentypen besiedeln kann. Für den angewandten Naturschutz ergibt sich hieraus ein Problem, zum Beispiel um die Frage, ob man eher alte Heckenstrukturen fördern sollte, weil darin viele Grasmückenarten in hoher Dichte vorkommen, oder ob eher jüngere Hecken naturschutzrelevant sind, weil darin die heckentypische und auf der Vorwarnliste (WITT et al. 1996) geführte Dorngrasmücke hohe Dichten erreicht.

Die optimale Hecke für Vögel $\mathrm{zu}$ finden oder $\mathrm{zu}$ gestalten ist ein nicht $\mathrm{zu}$ erreichendes Naturschutzziel. Sinnvoll ist es sicherlich, die Entwicklung eines breiten Angebots von Heckentypen in unterschiedlicher Ausprägung in der Landschaft anzustreben. Lokaltypische Hecken oder bestimmte Sonderformen, wie Wallhecken, Knicks oder Steinriegelhecken sollten jedoch in ihrer historisch gewachsenen (Nutzungs-) Form erhalten bleiben und nicht nach Gesichtspunkten maximaler Vogelabundanz verändert werden. 


\subsubsection{Dominanz von Vogelarten in Hecken mit Waldcharakter und Hecken mit weniger ausgeprägten Gehölzstrukturen}

Hecken zeigen strukturell und geobotanisch Übereinstimmungen mit Waldrandbiotopen (TisChler 1959, EllenBERG 1996). Ökophysiognomisch werden Hecken als 'doppelte Waldränder' bezeichnet (STREETER et al. 1985) und die Fauna weist viele Waldarten auf. Es stellt sich die Frage, ob die Brutvogelgemeinschaften auch von Waldvogelarten dominiert werden, bzw. ob einzelne Brutvogelarten durch ihre Habitatwahl unterschiedlichen Heckentypen zugeordnet und ob Waldarten anhand der Daten benannt werden können.

M: Die Heckenparameter 'alt', 'geschlossen', 'breit' und 'hoch' kennzeichnen Heckenstrukturen, die eher Waldcharakter besitzen, als junge, lückige, schmale oder niedrige Hecken. Entsprechend der gefundenen Unterschiede ihrer Dominanz (Tab. 7, Tab. 46, Tab. 47 und Tab. 48) sind die Vogelarten in Tab. 9 den vier betrachteten Parametern zugeordnet.

Tab. 9: Strukturwahl von Singvögeln in Hecken. Eingefärbte Flächen zeigen signifikante Bevorzugung einer Strukturausprägung der Hecken durch die betreffende Vogelart an (Z-Test mit Korrektur nach Bonferoni). Dunkle Flächen zeigen ausschließliche Bevorzugung von waldartigen, hell die von halboffenen Strukturen.

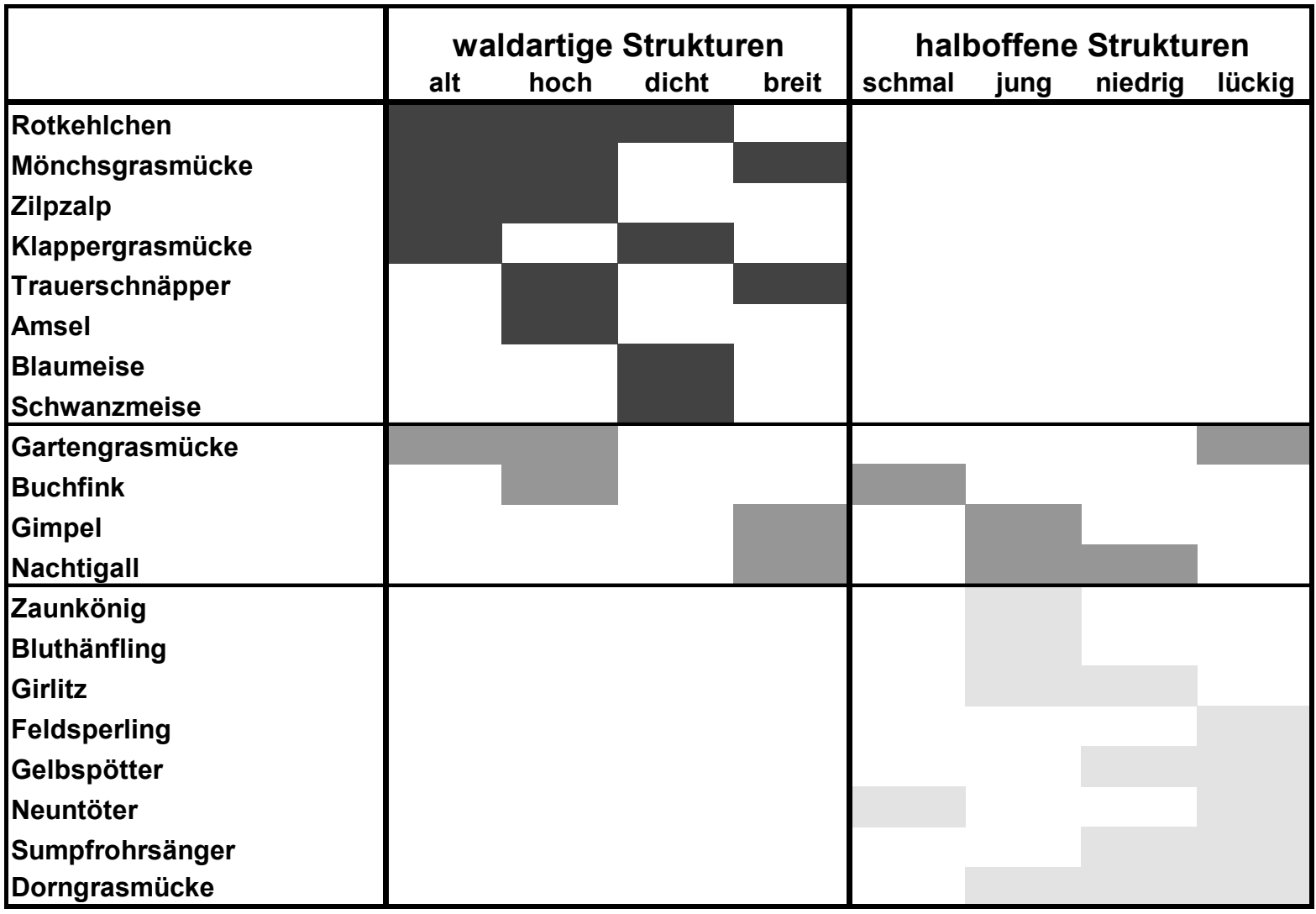

E: Jeweils acht Arten bevorzugen entweder nur waldartige Heckenstrukturen oder können ausschließlich den Strukturen halboffener Hecken zugeordnet werden. Bei den Waldarten sind mit Rotkehlchen und Mönchsgrasmücke zwei Arten vertreten, die in drei von vier Fällen 
signifikant häufiger in den waldähnlicheren Hecken vorkommen. Auf der anderen Seite bevorzugt die Dorngrasmücke eindeutig junge, niedrige und lückige, also halboffene Heckenstrukturen (vgl. Abb. 6). Arten wie Neuntöter, Sumpfrohrsänger (s. Tab. 8) oder Bluthänfling, Girlitz und Feldsperling nutzen ebenfalls gehölzärmere und offene Strukturen.

D: Für die 'Waldarten' in den Hecken ist anzumerken, dass es sich nicht um Waldbewohner im engeren Sinn handelt, sondern um Arten, die eher als eurytop zu bezeichnen sind. Die meisten Arten treten außerhalb der verschiedenen Waldtypen und Flurgehölzbeständen auch in Parks und Friedhöfen oder in Grünanlagen von Städten und Gemeinden auf und besiedeln somit ein breites Spektrum gehölzbestandener Lebensräume.

Unter den Arten, die offenere Heckenstrukturen bevorzugen sind einige samenfressende Arten, wie Bluthänfling, Feldsperling und Girlitz. Granivore Arten brauchen Zugang zu Krautsäumen und samentragenden Stauden, Wege und Störstellen auf Ackerflächen zur Nahrungssuche und bevorzugen somit insgesamt eher lockere Baum- und Gebüschbestände GLUTZ VON BLOTZHEIM \& BAUER 1997).

Vier Arten sind in beiden Strukturgruppen vertreten. Darunter ist auch der Buchfink, einer der häufigsten Brutvögel der Wälder Mitteleuropas (BAUER \& BERTHOLD 2000), der in dieser Studie in schmalen Hecken höhere Dominanzwerte erreichte als in breiten.

Die Auflistung der Arten in Tab. 9 umfasst nicht alle Programmvögel, weil nicht bei jeder Art Unterschiede in der Dominanz auftraten. Die Festlegung der Unterscheidungsgrenzen (z.B. niedriger oder höher als $8 \mathrm{~m}$ ) ergab sich nach ökologischen Gesichtspunkten, aber auch aus einer möglichst ausgeglichenen Verteilung der Stichproben. Bei einigen Arten könnte die Auftrennung der Strukturparameter auf anderem Niveau (z.B. niedriger oder höher als $4 \mathrm{~m}$ ) durchaus zu einem Unterschied ihrer Dominanz in einer der Höhenklassen führen. Im Rahmen dieser Studie konnte das jedoch nicht weiter untersucht werden.

Anhand der in dieser Studie nachgewiesenen unterschiedlichen Bevorzugung von Heckenstrukturen durch Vögel lassen sich waldadaptierte Arten und Bewohner halboffener Gehölzlebensräume unterscheiden. BLAB (1993) präsentiert eine Übersicht nach ZIMMERLI (1979), in der heckenbewohnende Vögel nach den bevorzugten Orten ihrer Nahrungswahl eingeteilt werden. Bei den 8 Waldarten dieser Studie überwiegt die Nahrungssuche innerhalb der Hecken. Nur die Amsel sucht vorwiegend außerhalb. Bei den 'Feldvögeln' (BLAB 1993) sind mit Bluthänfling, Girlitz, Feldsperling und Neuntöter die Hälfte der Arten, durch ihre Nahrungssuchorte außerhalb der Hecken, in stärkerer Weise von der Nutzung und Struktur umliegender Flächen abhängig, als die Waldarten. Damit kommt für viele Bewohner halb- 
offener Landschaften ein weiterer Faktor zu den eigentlichen Heckenstrukturen hinzu, der die Populationen der betreffenden Arten limitieren kann. Daraus folgt, dass bei einem Managementplan für Hecken auch die Struktur und Nutzung des Heckenumlandes Berücksichtigung finden muss.

\subsection{Bestandsdynamik}

Die Durchführung des Heckenprogramms der deutschen Vogelwarten diente auch als Vorläuferstudie eines in Deutschland mittlerweile etablierten Integrierten Monitorings für Singvogelpopulationen, abgekürzt IMS (BAIRLEIN et al. 2000).

Bei der Planung des Heckenprogramms war mit der Begrenzung der Laufzeit auf fünf Jahre von vorne herein klar, dass eine gut fundierte Darstellung und Analyse der Bestandsentwicklungen von Heckenbrütern nicht möglich sein würde. Ziel dieses Abschnitts ist die Dokumentation der Bestandsdynamik von Jahr zu Jahr, um anhand der Befunde verschiedene Vorgehen zur Analyse und Abbildung von Populationsverläufen darzustellen.

M: Es werden zwei Möglichkeiten der Darstellung vorgestellt: Erstens die Abbildung der jährlichen Fangsummen und Bestandsveränderungen zum Vorjahr (5.3.1) und zweitens die Abundanzveränderungen in Relation zum Gesamtfang über alle Untersuchungsjahre (5.3.2). Die erste Form ist die klassische Dokumentation von Bestandsverläufen über die Zeit. Dazu wurden die Fangzahlen jeweils auf die konstante Anzahl von 100 Fangtagen beszogen, um eine direkte Vergleichbarkeit der Resultate zwischen den jahren herzustellen. Der zweite Ansatz bietet die Möglichkeit, besonders auffällige Bestandsveränderungen in einem Jahr auszuweisen, indem durch eine Konfigurations-Frequenz-Analyse (KFA, nach BoRTZ et al. 1990) Abundanzveränderungen von Arten in Relation zum Gesamtfang festgestellt werden. Gerade in Programmen mit begrenzter Laufzeit und wenigen Probeflächen erscheint dieses Vorgehen sinnvoll. Die KFA zeigt Abundanzveränderungen auf der Grundlage von Abweichungen gegenüber einem Erwartungswert für ein angepasstes Alpha-Level von 0,01 an. Der Erwartungswert ergibt sich aus dem Verhältnis der Fangsumme der Art zur Fangsumme über alle Arten jeweils für den gesamten Untersuchungszeitraum, multipliziert mit der Grundgesamtheit (alle Fänge) des betrachteten Jahres. Man erhält art- und jahresspezifische Erwartungswerte, die die Fangsummen eines Jahres berücksichtigen. In

Tab. 12 sind alle Abundanzwerte, die außerhalb der Schranken des Erwartungswertes liegen, farbig unterlegt. 
Im Rahmen dieser Analyse wurden nur Daten aus Untersuchungsgebieten verwendet, die drei Jahre oder länger am Heckenprogramm beteiligt waren. Es wurden alle Programmvögel mit Ausnahme von Grünling und Star in die Analyse einbezogen. Für die Darstellung der 'Fangsummen und Bestandsveränderungen von Jahr zu Jahr' und der 'Fänglinge in Relation zum Gesamtfang' sind nur die Ergebnisse für Erstfänge von Altvögeln dargestellt und diskutiert. Die jeweiligen Tabellen mit den Angaben für die Erstfänge von Jungvögeln befinden sich im Anhang (Tab. 49 und Tab. 50).

Zur Beschreibung der Bestandsdynamik wurden alle Hecken einbezogen, in denen über den vollen zeitlichen Umfang der Heckenstudie nach standardisierter Methodik Vögel gefangen und individuell markiert wurden $(\mathrm{n}=21)$.

Tab. 10: Anzahl der durchschnittlichen Fangtage zur Beschreibung der Bestandsdynamik über N=21 Hecken.

\begin{tabular}{|l|c|c|c|c|c|}
\hline & 1994 & 1995 & 1996 & 1997 & 1998 \\
\hline Fangtage [n] & 228 & 254 & 254 & 256 & 248 \\
\hline Fangtage [ø] & 10,9 & 12,1 & 12,1 & 12,2 & 11,8 \\
\hline
\end{tabular}

Die ausgewählten Hecken zeigten in den Jahren 1995 bis 1997 eine beinahe konstante Anzahl der im Mittel durchgeführten Fangtage. Im ersten Jahr war die Fangaktivität noch sehr gering und im letzten Jahr fiel die Anzahl der Fangtage um einen halben Tag gegenüber den Vorjahren ab, was eine Veränderung von etwa $3 \% \mathrm{zu}$ den Vorjahren ausmacht. Aufgrund dieser geringen Schwankung in der Anzahl der Fangtage, wurde auf eine Korrektur mit einem Quotienten verzichtet. Prozentuale Veränderungen von 1994 auf 1995 werden unter Berücksichtigung des geringeren Aufwandes im ersten Untersuchungsjahr nicht angegeben.

\subsubsection{Fangsummen und Bestandsveränderungen}

E: In den Jahren 1995, 1996 und 1998 waren überwiegend positive Bestandsentwicklungen zu erkennen, während 1997 die meisten Arten sinkende Bestandszahlen aufwiesen (Tab. 11). Von 1995 auf 1996 war ein Anstieg der Fangzahlen fast aller Arten zu verzeichnen. Der Grünling zeigte 1994 einen Bestand, der über die folgenden Jahre immer geringer wurde. Das ging auf einen hohen Fangerfolg in einer Hecke zurück und war dort auf samenreiche Ansaat auf den angrenzenden Ackerflächen in dem betreffenden Jahr zurückzuführen.

1997 hatten 15 Arten Bestandsrückgänge. Bei acht Arten war die Anzahl der Fänglinge geringer als 1995. In den ersten zwei Jahren der Untersuchung wurden in 100 Fangtagen z.B. etwa 50 Amseln gefangen, 1996 sogar 67,9 und 1997 wurden im selben Zeitraum und in den 
gleichen Hecken weniger als 40 gefangen. Auch 1998 blieb der Fangerfolg bei unter 40 Individuen.

1998 war der Fangerfolg über die Arten sehr unterschiedlich. Bei den Drosselvögeln ist für die Singdrossel im zweiten Jahr in Folge ein Rückgang festzustellen, Rotkehlchen und Nachtigall dagegen erreichten die höchsten Fangwerte seit Programmbeginn. Bei den Höhlenbrütern Kohl- und Blaumeise war nach 1997 ein weiterer Bestandsrückgang festzustellen, während Weidenmeise und Star auf einem für die jeweilige Art hohen Niveau relativ stabil blieben.

Tab. 11: Fänge adulter Vögel als Anzahl auf 100 Fangtage, mit Angabe der Veränderung zum Vorjahr; n=21 Hecken ununterbrochener Bearbeitung; $\square=$ positive Populationsentwicklung, $\square=$ negative Populationsentwicklung, Veränderungen unter $10 \%$ nicht gekennzeichnet.

\begin{tabular}{|c|c|c|c|c|c|c|c|c|}
\hline Art & ad 94 & ad 95 & ad 96 & $\begin{array}{r}\% 1996 \\
\text { zu } 1995\end{array}$ & ad 97 & $\begin{array}{r}\% 1997 \\
\text { zu } 1996\end{array}$ & ad 98 & $\begin{array}{r}\% 1998 \\
\text { zu } 1997 \\
\end{array}$ \\
\hline Amsel & 49,7 & 52 & 67,9 & 30,6 & 37,2 & $-45,2$ & 38,8 & 4,3 \\
\hline Singdrossel & 8,4 & 16,3 & 27,6 & 69,3 & 19,9 & $-27,9$ & 15,1 & $-24,1$ \\
\hline Nachtigall & 0,6 & 5,6 & 12,8 & 129 & 10,2 & $-20,4$ & 23,3 & 128 \\
\hline Rotkehlchen & 17,3 & 47,5 & 58,9 & 24 & 48,7 & $-17,3$ & 59,7 & 22,6 \\
\hline Kohlmeise & 39,1 & 39,6 & 63,8 & 61,1 & 50 & $-21,6$ & 43,7 & $-12,6$ \\
\hline Blaumeise & 17,3 & 20,9 & 23,1 & 4,8 & 18,1 & $-21,6$ & 14,1 & -22 \\
\hline Weidenmeise & 3,4 & 4,6 & 5,8 & 26,1 & 7,5 & 29,3 & 7,3 & $-2,7$ \\
\hline Star & 3,4 & 6,1 & 8,2 & 34,4 & 79,7 & 872 & 75,2 & $-5,6$ \\
\hline Mönchsgrasmücke & 73,2 & 86,7 & 98,4 & 13,5 & 91,2 & $-7,4$ & 115,5 & 26,6 \\
\hline Gartengrasmücke & 34,6 & 45,9 & 59,3 & 29,2 & 59,3 & 0 & 58,7 & -1 \\
\hline Dorngrasmücke & 13,4 & 39,8 & 49,8 & 25,1 & 44,3 & -11 & 40,3 & -9 \\
\hline Klappergrasmücke & 21,8 & 25 & 31,3 & 25,2 & 22,6 & $-27,8$ & 26,2 & 15,9 \\
\hline Fitis & 19,6 & 40,3 & 38,7 & -4 & 34,1 & $-11,9$ & 60,7 & 78 \\
\hline Zilpzalp & 44,7 & 76,5 & 104,5 & 36,6 & 104.4 & $-0,1$ & 147,1 & 40.9 \\
\hline Goldammer & 15,6 & 32,7 & 42,8 & 30,9 & 19,9 & $-53,5$ & 34 & 70,9 \\
\hline Sumpfrohrsänger & 4,5 & 23 & 44,4 & 93 & 54 & 21,6 & 35 & $-35,2$ \\
\hline Neuntöter & 2,8 & 6,6 & 11,5 & 74,2 & 7,1 & $-38,3$ & 5,8 & $-18,4$ \\
\hline Heckenbraunelle & 27,9 & 44,9 & 57,2 & 27,4 & 44,3 & $-22,6$ & 48,1 & 8,6 \\
\hline Zaunkönig & 3,9 & 8,2 & 9,9 & 20,7 & 4,4 & $-55,6$ & 5,8 & 31,8 \\
\hline Gelbspötter & 3,9 & 10,2 & 18,9 & 85,3 & 16,4 & $-13,2$ & 18,9 & 15,2 \\
\hline Buchfink & 23,5 & 23 & 35,4 & 53,9 & 19 & $-46,4$ & 21,8 & 14,7 \\
\hline Grünling & 126,8 & 54,1 & 51 & $-5,8$ & 37,7 & $-26,1$ & 31,1 & $-17,5$ \\
\hline
\end{tabular}

Gartengrasmücke und Mönchsgrasmücke hielten von 1996 bis 1998 ein relativ stabiles und hohes Bestandsniveau, die Mönchsgrasmücke erreichte 1998 den höchsten Wert. Klapperund Dorngrasmücke hatten 1998 wieder eine Bestandszunahme, beide Arten erreichten das Niveau von 1995.

Bei den Jungvögeln ist die jahrweise Dynamik über die Arten weniger deutlich (Tab. 49). Von 1995 auf 1996 nahmen zehn Arten zu und acht ab. 1997 wurden bei zwölf Arten Zu- und bei fünf Arten Abnahmen festgestellt und 1998 hatten wieder zehn Arten einen Bestandsanstieg, neun nahmen ab. Nachtigall, Rotkehlchen und Heckenbraunelle hatten in 
keinem der Untersuchungsjahre eine negative Bestandsentwicklung mit einer Abweichung um mehr als $10 \%$ zum Vorjahr. Die Fangzahlen von Jungvögeln beim Grünling nahmen 1996, 1997 und 1998 ab.

D: Die Anzahl der Arten, die im direkten Vergleich zwischen den Jahren 1995 und 1998 eine negative Bilanz aufwiesen, ist mit $\mathrm{n}=7$ nicht sehr hoch, auch der numerische Unterschied bei einigen Arten (z.B. Singdrossel von 16,3 auf 15,1 Fänge je 100 Fangtage gefallen) ist gering. Dennoch ist festzustellen, dass mit dem Neuntöter eine Art darunter ist, die in Deutschland auf der Roten Liste geführt wird, weil für die Art in den letzten 25 Jahren (1970-1994) eine Bestandsabnahme von über $20 \%$ festgestellt wurde (WITT et al. 1996). Der für die Altvögel des Neuntöters im Heckenprogramm festgestellte Bestandsrückgang für die Jahre 1995 bis 1998 beträgt $12,1 \%$.

Mit der in dieser Arbeit gewählten Form der Darstellung von Bestandsdynamik wird die Populationsentwicklung im Vergleich zum Vorjahr sofort augenscheinlich. Zur Beurteilung der Dynamik bedarf es jedoch längerer Untersuchungszeiträume, um signifikante Bestandstrends von natürlichen Popualtionsschwankungen unterscheiden zu können.

\subsubsection{Abundanzveränderungen einzelner Arten in Relation zum Gesamtfang}

Die Wiedergabe von Fangzahlen und deren Veränderung ist nur bei vollkommen gleichem Aufwand sinnvoll. In Abschnitt 4.5.1 wurde mit einem Wert von 'Fänglinge auf 100 Fangtage' gerechnet. Abundanzveränderungen wurden auf $10 \%$-Niveau angezeigt. Es können hierbei aber immer nur Veränderungen zur Situation im Vorjahr herangezogen werden. Dabei bleibt ungeklärt, ob und wann Abundanzveränderungen signifikant von einem aus allen Werten errechneten Erwartungswert abweichen.

E: Für die Mehrzahl der Programmarten lagen die gefundenen Abundanzen im Bereich der oberen und unteren Schranken um die berechneten Erwartungswerte. Bei 15 Arten (von 36) wurden signifikante Abundanzveränderungen festgestellt (farbig unterlegte Felder in

Tab. 12). Bei fünf Arten (Amsel, Gelbspötter, Goldammer, Kohlmeise und Rotkehlchen) überwogen Jahre mit signifikant weniger Fänglingen. Eine positive Entwicklung der Fangbilanz liegt bei Nachtigall, Zilpzalp und mit Einschränkungen beim Trauerschnäpper vor.

D: Während für Gelbspötter und Rotkehlchen die Ursache für zwei Jahre mit signifikant weniger Fänglingen darin liegen kann, dass 1994 (das Jahr niedrigster Fangzahlen der Arten) noch nicht in allen Probeflächen in vollem Umfang gearbeitet werden konnte, ist zu beachten, 
dass die Amsel über die Jahre hinweg im Fangvolumen abgenommen hat. Dazu kommt, dass zwei Jahre mit überdurchschnittlich wenigen Fängen aufeinander folgten. Das war bei keiner anderen Art zu beobachten. Negative Tendenz über die Jahre war auch bei Kohlmeise und Buchfink zu erkennen. Beide Arten zeigten insgesamt starke Jahresschwankungen der Fangzahlen, was ein Hinweis auf die variierende Fängigkeit beider Arten sein kann.

Tab. 12: Ergebnis der Konfigurations-Frequenz-Analyse (KFA) zu Abundanzveränderungen der Altvögel; $\square$ = signifikant niedrigere, $\square=$ signifikant höhere Fangzahl.

\begin{tabular}{|c|c|c|c|c|c|c|c|}
\hline & Art & 1994 & 1995 & 1996 & 1997 & 1998 & gesamt \\
\hline 1 & Amsel & 152 & 101 & 166 & 76 & 80 & 575 \\
\hline 2 & Baumpieper & 2 & 0 & 5 & 1 & 1 & 9 \\
\hline 3 & Blaumeise & 37 & 42 & 58 & 34 & 34 & 205 \\
\hline 4 & Bluthänfling & 2 & 5 & 5 & 1 & 1 & 14 \\
\hline 5 & Buchfink & 73 & 75 & 124 & 45 & 54 & 371 \\
\hline 6 & Dorngrasmücke & 77 & 98 & 108 & 105 & 82 & 470 \\
\hline 7 & Feldschwirl & 0 & 2 & 2 & 1 & 3 & 8 \\
\hline 8 & Feldsperling & 37 & 39 & 31 & 53 & 24 & 184 \\
\hline 9 & Fitis & 88 & 126 & 119 & 93 & 74 & 500 \\
\hline 10 & Gartenbaumläufer & 2 & 3 & 3 & 4 & 2 & 14 \\
\hline 11 & Gartengrasmücke & 117 & 114 & 148 & 124 & 113 & 616 \\
\hline 12 & Gartenrotschwanz & 3 & 13 & 16 & 11 & 8 & 51 \\
\hline 13 & Gelbspötter & 16 & 38 & 49 & 33 & 40 & 176 \\
\hline 14 & Gimpel & 19 & 11 & 21 & 12 & 6 & 69 \\
\hline 15 & Girlitz & 2 & 4 & 8 & 8 & 11 & 33 \\
\hline 16 & Goldammer & 67 & 96 & 106 & 51 & 85 & 405 \\
\hline 17 & Grauschnäpper & 1 & 7 & 7 & 2 & 5 & 22 \\
\hline 18 & Grünling & 250 & 120 & 125 & 66 & 36 & 597 \\
\hline 19 & Heckenbraunelle & 86 & 110 & 139 & 88 & 106 & 529 \\
\hline 20 & Kernbeißer & 11 & 21 & 6 & 8 & 7 & 48 \\
\hline 21 & Klappergrasmücke & 61 & 71 & 70 & 46 & 48 & 296 \\
\hline 22 & Kleiber & 3 & 3 & 3 & 2 & 0 & 11 \\
\hline 23 & Kohlmeise & 127 & 55 & 155 & 99 & 70 & 506 \\
\hline 24 & Mönchsgrasmücke & 272 & 253 & 254 & 243 & 271 & 1293 \\
\hline 25 & Nachtigall & 8 & 9 & 25 & 15 & 33 & 90 \\
\hline 26 & Neuntöter & 18 & 17 & 27 & 14 & 10 & 86 \\
\hline 27 & Rotkehlchen & 59 & 130 & 183 & 115 & 125 & 612 \\
\hline 28 & Schwanzmeise & 4 & 15 & 12 & 5 & 6 & 42 \\
\hline 29 & Singdrossel & 27 & 44 & 70 & 44 & 29 & 214 \\
\hline 30 & Star & 10 & 14 & 26 & 179 & 159 & 388 \\
\hline 31 & Stieglitz & 11 & 3 & 9 & 7 & 6 & 36 \\
\hline 32 & Sumpfmeise & 15 & 2 & 5 & 3 & 3 & 28 \\
\hline 33 & Sumpfrohrsänger & 48 & 71 & 115 & 93 & 69 & 396 \\
\hline 34 & Trauerschnäpper & 3 & 5 & 10 & 4 & 13 & 35 \\
\hline 35 & Wacholderdrossel & 2 & 3 & 7 & 4 & 3 & 19 \\
\hline 36 & Weidenmeise & 12 & 18 & 19 & 15 & 9 & 73 \\
\hline 37 & Zaunkönig & 17 & 20 & 26 & 10 & 13 & 86 \\
\hline 38 & Zilpzalp & 158 & 231 & 288 & 221 & 306 & 1204 \\
\hline & Summe ohne Nr. 18 u. 30 & 1637 & 1855 & 2399 & 1690 & 1745 & 9326 \\
\hline
\end{tabular}

Im Vergleich mit den Ergebnissen aus Tab. 11 fällt auf, dass es kein Jahr mit deutlich positiver oder negativer Tendenz über alle Arten gibt. Mit Hilfe einer KFA werden nur sehr deutliche Abweichungen innerhalb einer Art zu einem Zeitpunkt (hier: ein Jahr) ermittelt. Eine kontinuierliche Bestandsabnahme wird nicht angezeigt (z.B. Singdrossel und Neuntöter). 
Zwei durch die KFA ausgewiesene, signifikant schlechte Jahre der Amsel sind bei der Darstellung in Tab. 11 nicht aufgefallen. Dort wird nur der starke Rückgang von 1997 abgebildet. Erst bei Berücksichtigung der insgesamt gefangenen Individuen der Art wird deutlich, dass die Amsel zwei Jahre hintereinander sehr geringe Fangzahlen aufwies.

Unter Berücksichtigung schwankender Fangerfolge in den unterschiedlichen Jahren ist eine KFA gut geeignet, um als Instrument zur Indizierung von signifikant abweichenden Fangergebnissen herangezogen zu werden. Die Stärke der Methode liegt darin, dass das Fangergebnis einer Art jeweils auf die Grundgesamtheit aller Fänge und die Fangergebnisse der betrachteten Art über alle Fangjahre bezogen wird.

Ein allmählicher Rückgang oder Anstieg von Populationszahlen über die Zeit wird durch diese Methodik nicht ausgewiesen. Die KFA kann somit die Erfassung der flächen- und zeitraumbezogenen Fangsummen nicht ersetzen, sondern diese nur ergänzen und eignet sich besonders bei Programmen mit kurzer Laufzeit.

\subsection{Fangphänologie}

Ziel des standardisierten Netzfangs zur Brutzeit ist es, über die Fangzahlen von Altvögeln den Brutbestand abzuschätzen und über die Fangzahlen von Jungvögeln und deren Verhältnis zu den Adulten den relativen Bruterfolg in Hecken zu erheben. Im Frühjahr muss damit gerechnet werden, dass sich einige der Fänglinge nicht als Brutvögel in den Hecken ansiedeln, sondern diese wieder verlassen. Ebenso ist anzunehmen, dass nicht alle in der Hecke gefangenen Jungvögel dort auch ausgeflogen sind.

M: Um Durchzügler von den territorialen Individuen zu trennen, wurden die Fangzahlen nach Dekaden aufgetragen, weil sich Durchzugsphänomene oft an sprunghaft veränderten Fangzahlen erkennen lassen. Der Vergleich der Fangzahlen von Dekade zu Dekade stellte in dieser Studie die kleinste Bezugseinheit zur Beschreibung der Fangentwicklung dar. Für die Berechnung von Reproduktionsindizes wurden auf der Basis der in diesem Kapitel dargestellten Fangphänologien Zeitabschnitte bestimmt, in denen die Anzahl von durchziehenden Altvögeln oder dismigrierenden Jungvögeln minimal ist.

Es wurden alle durchgängig bearbeiteten Hecken und die Flächen, auf denen mindestens 10 Fangtage pro Jahr durchgeführt worden sind, einbezogen. Insgesamt flossen Daten von 1.391 Fangtagen aus 25 Hecken in die Auswertung ein (Tab. 13).

E: Im Mittel wurden je Dekade 99,4 (SD=8,7; SF=2,3) Fangtage durchgeführt (Tab. 13). 
Tab. 13: Anzahl der Fangtage je Dekade zur Beschreibung der Fangsummen über N=25 Hecken, mit Angabe der Abweichung je Dekade vom Mittelwert über alle Dekaden.

\begin{tabular}{|l|rrr|rrr|rrr|rrr|r|r|}
\hline & \multicolumn{3}{|c}{ April } & \multicolumn{3}{|c|}{ Mai } & \multicolumn{3}{c|}{ Juni } & \multicolumn{3}{c|}{ Juli } & August \\
Dekade & 1 & 2 & 3 & 4 & 5 & 6 & 7 & 8 & 9 & 10 & 11 & 12 & 13 & 14 \\
\hline Anzahl Fangtage & 83 & 109 & 102 & 96 & 102 & 94 & 93 & 102 & 106 & 93 & 112 & 98 & 112 & 89 \\
\hline Abweichung vom MW & $-16,5$ & 9,7 & 2,6 & $-3,4$ & 2,6 & $-5,4$ & $-6,4$ & 2,6 & 6,6 & $-6,4$ & 12,7 & $-1,4$ & 12,7 & $-10,5$ \\
\hline
\end{tabular}

Starke Abweichungen vom Mittelwert traten besonders zu Beginn der Fangperiode bis Mitte April und gegen Ende der Fangzeit ab Mitte Juli auf. Dekaden mit wenigen Fangtagen wechseln mit Dekaden überdurchschnittlich vieler Fangtage ab. Für die Darstellung von Fangphänologien wurden die Fangsummen um die Abweichung der Fangtage auf den Mittelwert korrigiert. Zur Darstellung der Fangmuster wurden Arten mit mindestens 150 Erstfängen ausgewählt, Grünling und Star wurden aus bereits erwähnten Gründen nicht in die Darstellung (Abb. 7) mit aufgenommen.

Die Fangzahlen adulter und juveniler Vögel sind nicht gleichmäßig über die Dekaden verteilt (Abb. 7). Bei der Summierung der häufigsten 18 Heckenbrüter tritt ein Maximum adulter Fänglinge gegen Ende April auf. Ab Mitte Juni pendelt die Fangzahl um einen Wert bei 500 Erstfängen.

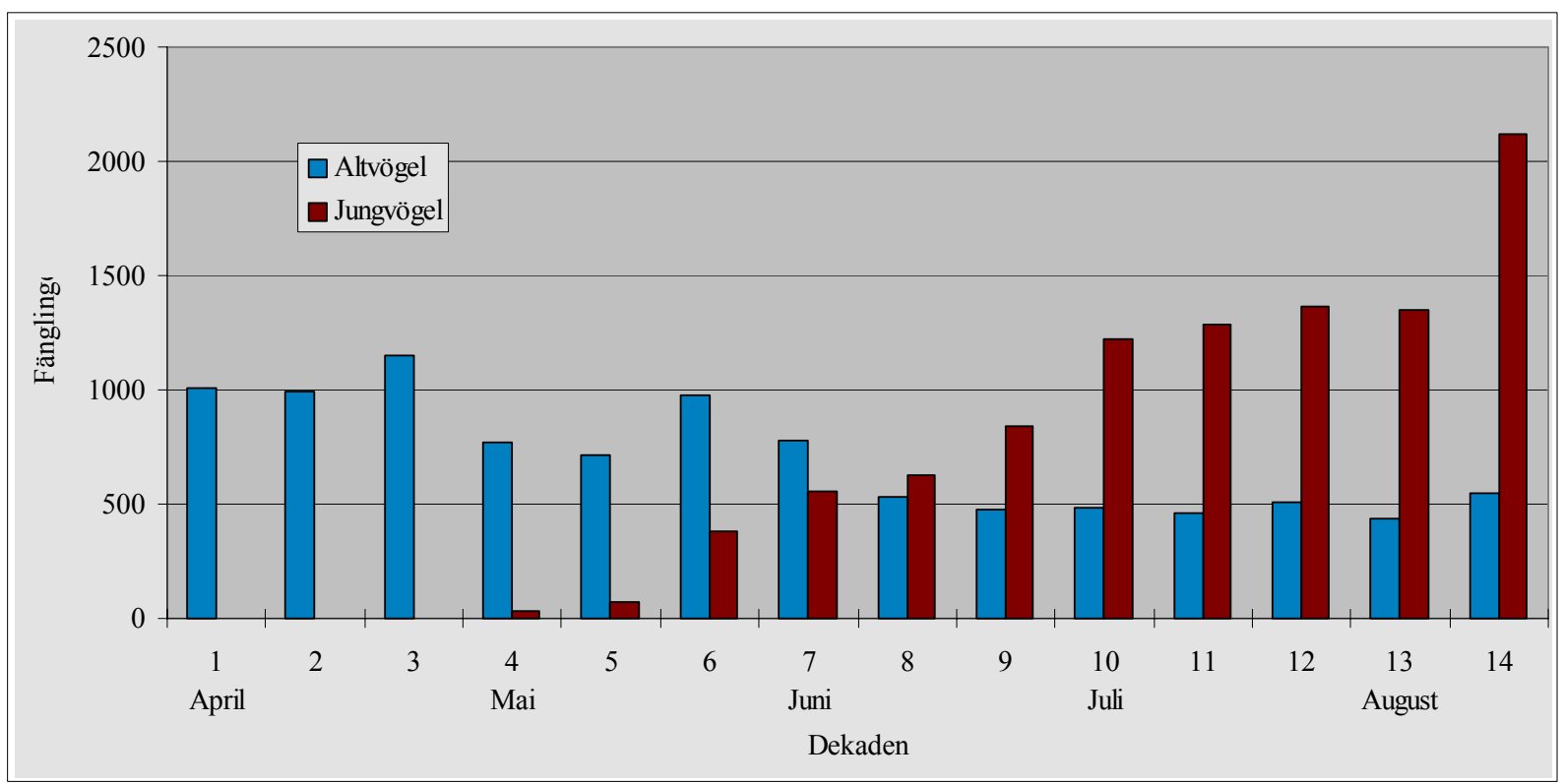

Abb. 7: Dekadensummen beringter Altvögel (nd/F) und Jungvögel (dj) aus 25 Hecken in fünf Jahren Heckenprogramm. Für n=18 Arten, Daten dem Fangaufwand in den Dekaden entsprechend angepasst.

Nach dem Auftreten der ersten Jungvögel ab Mai nimmt deren Anzahl bis zum Ende der Beringungszeit (etwa Mitte August) von fast jeder Dekade auf die nächste zu. Zwischen Anfang Juli und Anfang August ist die Zunahme nur noch gering. Die Entwicklung der Fangzahlen zeigt bis dahin den Verlauf einer Sättigungskurve. In der zweiten August-Dekade ist ein sprunghafter Anstieg von etwa 1400 auf über 2100 Jungvögel zu erkennen. 
D: Der Fang von Jungvögeln setzt im Mai mit sehr geringen Fangzahlen ein. Es konnten nur etwa 100 Individuen bis zum 20. Mai beringt werden, was ein Hinweis auf geringen Bruterfolg zu Beginn der Brutperiode in Hecken ist.

Sowohl das Maximum der Adulten im April als auch das der Jungvögel im August sind durch durchziehende bzw. dissmigrierende Individuen zu erklären. Die Fangmaxima stimmen mit den dem Auftreten vieler Arten zu den Zugzeiten an den deutschen Fangstationen Helgoland, Greifswalder Oie oder Oldeoog (hier befinden sich keine Bruthabitate für die Mehrzahl der betrachteten Arten) überein (vgl. BAIRLEIN 1997). Ein weiterer Hinweis auf einen hohen Anteil durchziehender Altvögel ist die Gesamtanzahl von Erstberingungen im Vergleich mit den Ergebnissen der Kartierungen. So kommen auf etwa 300 Brutpaare der Mönchsgrasmücke mehr als fünfmal so viele Beringungen von Altvögeln (vgl. Tab. 2 und Tab. 45).

\subsubsection{Fangphänologie einzelner Arten}

Die Fangphänologien adulter Mönchs- und Gartengrasmücken zeigen einen vergleichbaren Verlauf, wenn auch das Muster der Gartengrasmücke, aufgrund späteren Eintreffens in den Brutgebieten, um zwei Dekaden nach hinten verschoben ist (Abb. 8). In der dritten Dekade nach Erscheinen der ersten Vögel erreicht die Anzahl beringter Altvögel ein Maximum. Die niedrigsten Beringungszahlen adulter Vögel folgen vier bis sechs Dekaden darauf, bei der Mönchsgrasmücke im Juni, bei der Gartengrasmücke Anfang Juli. Bei letztgenannter Art steigen danach die Beringungszahlen wieder stetig an.
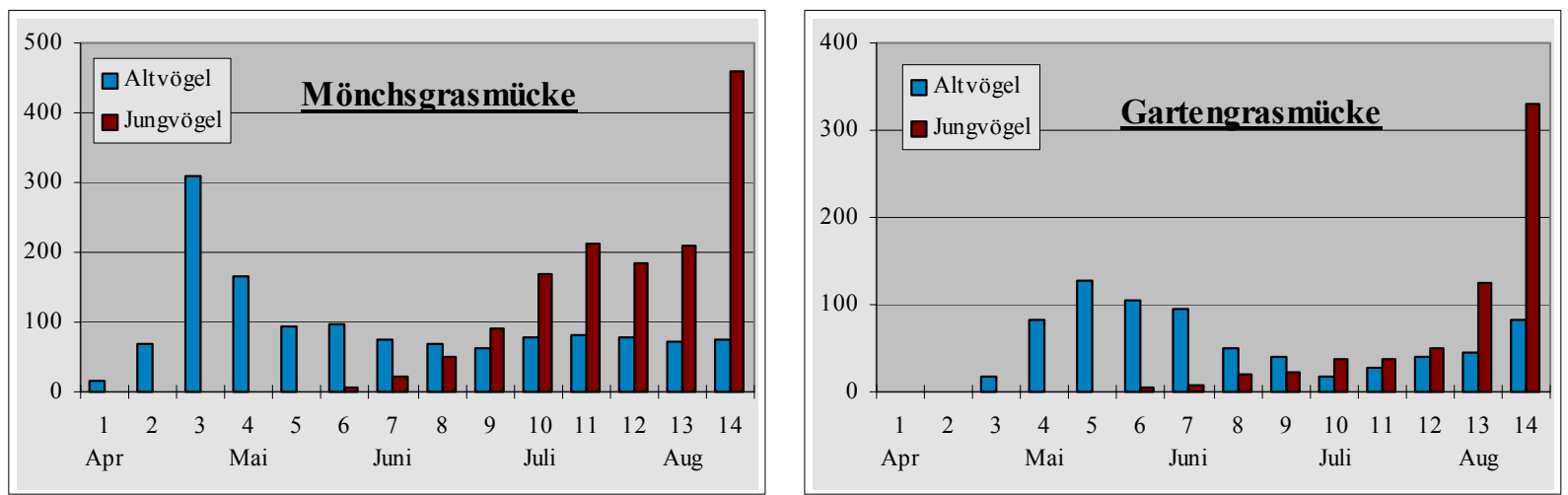

Abb. 8: Dekadensummen beringter Mönchs- und Gartengrasmücken aus 25 Hecken in fünf Jahren Heckenprogramm. Daten dem Fangaufwand entsprechend angepasst.

Als Langstreckenzieher verweilt die Gartengrasmücke kürzer im Brutgebiet als die Mönchsgrasmücke und es erscheint plausibel, dass der Anstieg der Fangzahlen von Adulten im August auf einsetzende Zugbewegungen zurückzuführen ist. Nach HELBIG \& DiERSCHKE (2000) erreicht die Gartengrasmücke Ende August in Süddeutschland ihren Durchzugsgipfel. 
Der Verlauf der Juvenilenanzahlen zeigt kein ausgeprägtes Juli-Maximum. Der Anstieg verläuft ab Ende Mai (Mitte Mai ein Jungvogel als juvenil vermerkt, sicher ein Irrtum) stetig aber flach ansteigend, bis die Anzahl der jungen Gartengrasmücken in den Hecken im August exponentiell ansteigt. Auf Helgoland erfolgt dieser Anstieg zeitgleich von der 45. auf die 46. (14.-18- August) Pentade, in Galenbeck, wo die Art auch brütet, erfolgt ein Anstieg bereits in der letzten Juli-Dekade (BAIRLEIN 1997).

Die Mönchsgrasmücke zeigt, im Gegensatz zur Gartengrasmücke, für die Beringungen an Jungvögeln ein relatives Maximum in Dekade 11 (Mitte Juli, Abb. 8). Der kontinuierliche Anstieg der Fangzahlen bis dahin und die gute Abgrenzung gegen den Höchststand der Fangzahlen junger Mönchsgrasmücken einen Monat später, legt den Schluss nahe, dass das relative Maximum durch die Beringung von urban erbrüteten und dispergierenden Individuen zustande kommt. Der plötzliche Anstieg in Dekade 14 dürfte dagegen eher auf hohe Anzahlen durchziehender Vögel zurückzuführen sein.

Am Beispiel dieser Grasmücken-Arten wird die Bedeutung der Hecken für eine Vielzahl von Singvögeln deutlich: Hecken sind bis in die Brutzeit hinein und dann wieder zu deren Ende wichtige Habitate in der Zugzeit, was den bei fast allen Arten vorhandenen Frühjahrsgipfel der Altvögel und die stetig steigende Anzahl an Jungvögeln im Sommer und Spätsommer (Dismigrationsbewegungen und einsetzender Zug in die Überwinterungsgebiete) erklärt.
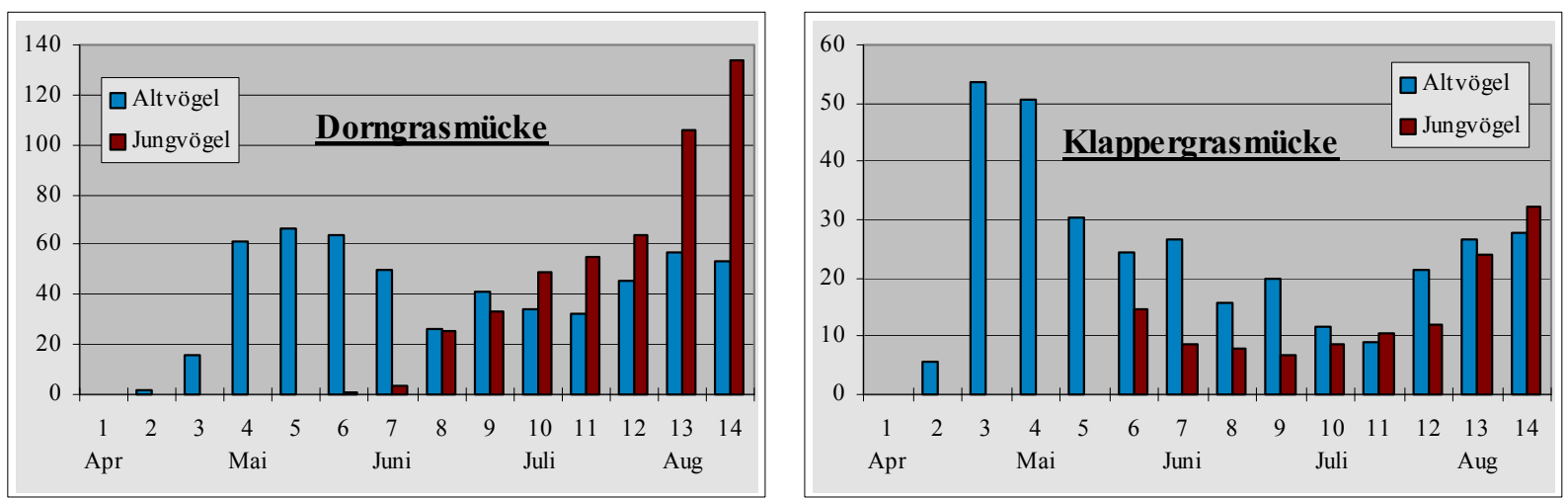

Abb. 9: Dekadensummen beringter Dorn- und Klappergrasmücken aus 25 Hecken in fünf Jahren Heckenprogramm. Daten dem Fangaufwand entsprechend angepasst.

E: Nach den Maxima im Frühjahr steigen die Fangzahlen von adulten Dorn- und Klappergrasmücken ab Ende Juli wieder an (Abb. 9). Wenn auch weit weniger auffällig als die Ankunft in den Brutgebieten scheint auch der Herbstzug der Altvögel über die Nutzung von Hecken stattzufinden.

D: Typischerweise ist der Höhepunkt durchziehender Altvögel von Langstreckenziehern (Fitis, Gelbspötter, Dorngrasmücke, Gartengrasmücke, u.a.) dem der Jungvögel vorgelagert 
(BERTHOld 1990), die Adulten verlassen die Brutgebiete vor den Jungen. Dieses scheint auch für Singdrossel und Mönchsgrasmücke zu gelten, deren mitteleuropäischen Bestände eher den Kurzstreckenziehern zuzurechnen sind. Ein weiterer Grund für den Anstieg der Fangzahlen von Altvögeln im Juli-August kann darin liegen, dass mit der Aufgabe intensiver Revierverteidigung das großen Nahrungsangebot für eine größere Anzahl von Individuen nutzbar wird. Auch in diesem Fall wäre der Anstieg der Fangzahlen auf eingewanderte Individuen zurückzuführen.

Die Nachtigall ist ein Beispiel für eine Art, bei der die Altvögel nach der Brutzeit in sehr kurzem Zeitintervall mit vielen Individuen in den Hecken festgestellt werden und somit als Durchzügler erscheinen. Neben einem relativen Maximum durch Fangzahlen adulter Vögel im Frühjahr weist das Diagramm auch einen deutlichen Höhepunkt zum Wegzug auf (Dekaden 12 und 13, Abb. 10). Die Beringungszahlen der Art zeigen auch bei den Jungvögeln eine deutliche Zweigipfeligkeit (Dekade 9/10 bzw. 14). Hierbei sind die hohen Beringungszahlen Ende Juni und Anfang Juli wahrscheinlich auf Jungvögel zurückzuführen, die in den jeweiligen Hecken oder der näheren Umgebung erbrütet wurden. Der Anstieg juveniler Nachtigallen ab August könnte mit dem Durchzug von Vögeln aus nördlicheren Regionen erklärt werden. Da die Nachtigall in der Regel bis Mitte September die Brutgebiete verlassen hat (BERTHOLD et al. 1991), schlägt sich der Wegzug der Art sowohl von Altvögeln als auch von Jungvögeln in den Fangsummen des Heckenprogramms nieder. Dieses ist in Ansätzen auch bei anderen Langstreckenziehern zu beobachten, insbesondere wenn die Arten eine vergleichsweise kurze Verweildauer im Brutgebiet haben, wie etwa Sumpfrohrsänger (ca. 55 Tage, BezzEL 1993) und Gelbspötter (vgl. Abb. 10).

Der Fitis (Langstreckenzieher) wies ebenfalls deutlich erhöhte Beringungszahlen um Mitte Juli auf. Ein sprunghafter Anstieg der Beringungszahlen bei den Jungvögeln erfolgt Mitte August (Abb. 10). In der dritten Aprildekade zeigte der Fitis ein Maximum. Dieser Befund steht im Widerspruch zu Helbig \& DierschKe (2000), die für diese Art Durchzugsgipfel in der ersten (Süddeutschland) bzw. zweiten Dekade im Mai (Norddeutschland) angeben. Auch nach BAIRLEIN (1997) hat die Art auf der Greifswalder Oie (Norddeutschland) ein absolutes Fangmaximum in der zweiten Dekade im Mai. 

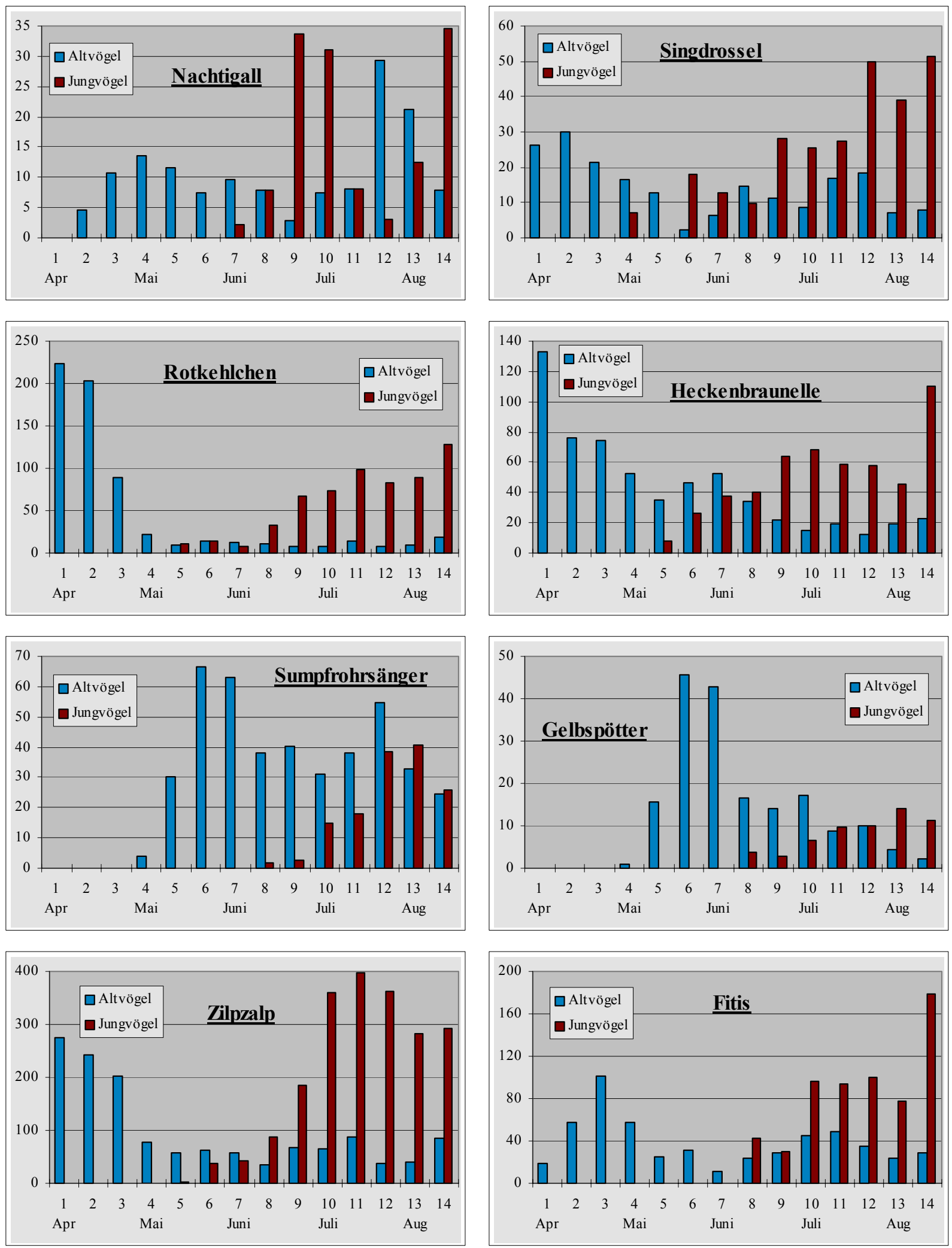

Abb. 10: Dekadensummen ziehender Singvogelarten aus 25 Hecken in fünf Jahren Heckenprogramm. 

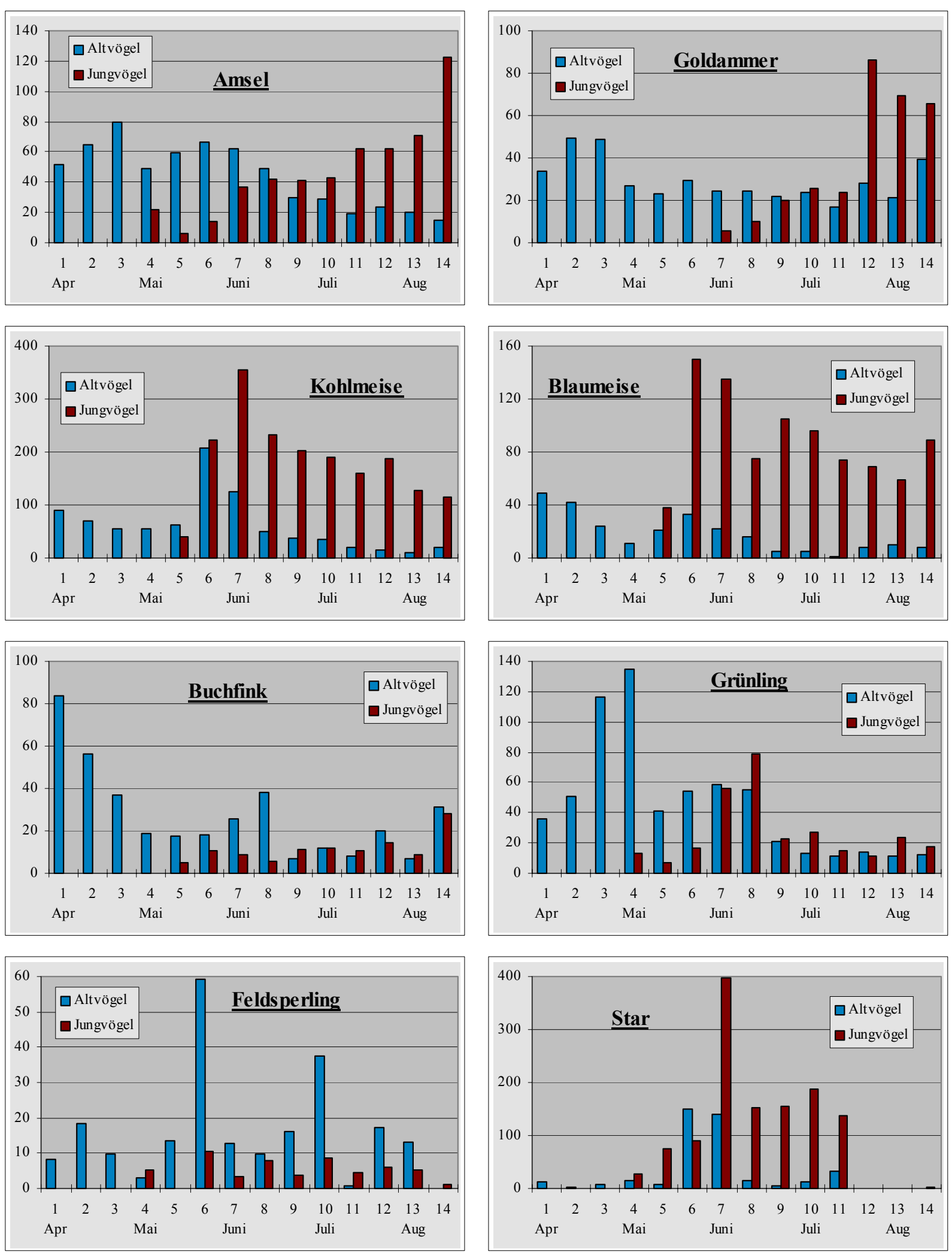

Abb. 11: Dekadensummen beringter Standvögel und Teilzieher aus 25 Hecken in fünf Jahren Heckenprogramm.

E: Die Anzahlen der nach Dekaden aufgeteilten Erstfänge der im Heckenprogramm beringten Zugvögel (z.B. Grasmücken, Nachtigall, Laubsänger, Sumpfrohrsänger und Gelbspötter) zeigen ein vergleichbares Muster: Nach Ankunft der ersten Adulten im Frühjahr wird zumeist 3-4 Dekaden später ein Durchzugsmaximum erreicht. Die Beringungszahlen für adulte Tiere 
gehen dann zurück und bleiben auf einem mehr oder weniger konstanten Niveau, um im Juli/August durch einsetzende Dismigration wieder anzusteigen oder ein zweites relatives Maximum zu erreichen. Dieses wird in Abb. 7 durch die Summierung vieler Arten überlagert, ist aber in Diagrammen einzelner Arten zu erkennen, z.B. Nachtigall, Sumpfrohrsänger und Fitis (Abb. 10), Dorn- und Klappergrasmücke (Abb. 9).

Mit Abschluss der ersten Brut tauchen ab Mitte Mai (Amsel und Singdrossel noch früher) die ersten Jungvögel auf, die dann bei den meisten Arten stetig ansteigende Beringungszahlen aufweisen oder mit relativ konstanten Fangzahlen bis in den Juli hinein bleiben können. Zumeist übertrifft die Anzahl der Juvenilen die der Adulten erstmals im Juni oder Juli. Bei einigen Arten ist mit Beginn der Augustdekaden (13. und 14.) ein sprunghafter Anstieg der Juvenilen zu beobachten (z.B. Amsel und Goldammer, Abb. 11).

\subsection{Indizierung des relativen Bruterfolgs}

Ein wichtiges Ziel des Heckenprogramms ist die Klärung der Frage nach dem Bruterfolg der Arten in diesem Lebensraum und seiner nahen Umgebung. Untersuchungen haben ergeben, dass der Bruterfolg von Heckenbrütern sehr gering sein kann (BAIRLEIN \& SoNNTAG 1994) und dass von der Besiedlungsdichte nicht auf den Bruterfolg und die damit verbundene 'ökologische Qualität' eines Lebensraumes geschlossen werden kann (VICKERY et al. 1992b).

\subsubsection{Festlegung der Zeitintervalle zur Berechnung von Bruterfolgs-Indizes}

Neben der direkten Kontrolle des Bruterfolgs an Nestern bietet sich die Möglichkeit einer Indizierung des jährlichen Bruterfolgs über das Verhältnis von Jungvögeln zu Adulten im standardisierten Netzfang. Die Fangzahlen vieler Brutvögel in den Hecken unterliegen aufgrund von Durchzugszeiten im April bis in den Mai sowie ab Ende Juli oder August Schwankungen, die das Verhältnis zwischen Juvenilen und Adulten beeinflussen. Dadurch würden Veränderungen im Bruterfolg eventuell überlagert und eine Interpretation der Ergebnisse schwierig. Zur besseren Interpretierbarkeit des 'relativen Bruterfolgs' werden im Folgenden artspezifische Betrachtungszeiträume ermittelt, die Dekaden mit hohen Durchzugszahlen ausblenden.

M: Die Festlegung der Zeitintervalle zur Berechnung von Bruterfolgs-Indizes ergibt sich aus der Phänologie der Arten. Diese wird tabellarisch zusammengefasst. Unter 'Max nd' wird die Dekade mit dem Durchzugsmaximum der Adulten angegeben. Es folgt das Auftreten der ersten Jungvögel (erste dj) und der Zeitpunkt, an dem die Anzahl der neu beringten Jungvögel zum ersten Mal die der Altvögel übertrifft (dj>nd). Rund eingeklammerte Angaben unter 
'Max nd' schließen ein früher liegendes Maximum außerhalb des Untersuchungszeitraums nicht aus. Die Zeiträume, die für die Berechnung des Verhältnisses der Jungvögel zu den Altvögeln herangezogen werden, werden unter 'Zug minimal' angegeben. Das zu betrachtende Zeitfenster kann durch ein (Durchzugs-) Maximum der Altvögel im Frühjahr oder durch einen starken Anstieg der Beringungszahlen (>50\%) bei den Jungvögeln im August eingegrenzt werden. Ziel ist immer der Ausschluss von einer hohen Anzahl durchziehender Individuen. Unter Berücksichtigung der ermittelten Fangmuster (Abb. 8 bis Abb. 11), der Brutökologie (Brutphänologie, Kompensation von Nestverlusten) und der artspezifischen Zugzeiten werden die Zeitfenster für die Berechnung von Bruterfolgsindizes abgeleitet. Die artspezifische Festlegung dieser Zeitspanne mit minimalen Einflüssen aus Zugbewegungen dient als Grundlage zur Berechnung eines Indexes aus dem Quotienten der Summe beringter Jungvögel und der Summe adulter Erst- und Kontrollfänge.

$\underline{\mathrm{E}+\mathrm{D}}$ : Im Folgenden werden die Argumente für die Festlegung des Zeitintervalls 'Zug minimal' dargelegt und im Zusammenhang diskutiert. In Tab. 14 sind die wichtigsten phänologischen Daten aus den Diagrammen in Abb. 8 bis Abb. 11 für die 20 häufigsten Arten zusammengefasst.

Mönchsgrasmücke: Nach dem Durchzugsmaximum adulter Vögel Ende April wird der Betrachtungszeitraum bis zum Ende der ersten Dekade im August ausgedehnt. Erst danach erfolgt ein Anstieg der Beringungszahlen an Jungvögeln von knapp 250 auf über 400 Individuen. BAIRLEIN (1978) konnte an einer Population in Süddeutschland zeigen, dass bereits ab Mitte Juli verstärkt junge Mönchsgrasmücken aus Nachbargebieten zuwandern. Dieses spiegelt sich auch in den Beringungszahlen ab Dekade 11 (Abb. 8) wider. Das Erscheinen der ersten Jungvögel zeichnet sich zunächst nur sehr zögerlich ab (Dekade 6 und 7, entsprechend Ende Mai bis Anfang Juni). Das lässt vermuten, dass eine Vielzahl von Gelegen der Erstbrut in den Hecken verloren geht, was in der Regel zur Anlage von Nachgelege führt. Die Mehrzahl daraus ausfliegender Jungvögel könnte dann erst im Juli nachgewiesen werden (s. relatives Maximum der Jungvögel Mitte Juli). Vor dem Hintergrund der Möglichkeit bereits einwandernder Jungvögel aus benachbarten Gebieten stellt das gewählte Zeitfenster einen Kompromiss dar, der darauf abzielt, möglichst viele Jungvögel zu erfassen, die in den Hecken erbrütet worden sind (Einschluss der Dekaden im Juli). Gleichzeitig soll der Einfluss von Fänglingen aus Nachbargebieten minimiert werden (Abtrennung der letzten Dekade im August). 
Tab. 14: Phänologiedaten der häufigsten Heckenvögel (n=20) aus 25 Hecken und Bestimmung der Dekaden mit geringem Einfluss der Fangergebnisse durch Migration (Zug minimal). Dekaden entsprechend der Festlegung in Tab. 3, weitere Abk. im Text.

\begin{tabular}{|l|cccc|}
\hline \multicolumn{1}{|c|}{ Art } & Max nd & erste dj & erstmals dj>nd & Zug minimal \\
\hline Mönchsgrasmücke & 3 & 6 & 9 & $4-13$ \\
Gartengrasmücke & 5 & {$[5 ?] 6$} & 10 & $6-12$ \\
Dorngrasmücke & 5 & 6 & 10 & $6-12$ \\
Klappergrasmücke & 3 & 6 & 11 & $5-12$ \\
\hline Nachtigall & 4 & 7 & 9 & $5-11$ \\
Singdrossel & 2 & 4 & 6 & $3-11$ \\
Rotkehlchen & 2 & 5 & $5(8)$ & $3-10$ \\
Heckenbraunelle & $(1)$ & 5 & 8 & $4-13$ \\
\hline Sumpfrohrsänger & 6 & 8 & 13 & $8-14$ \\
Gelbspötter & 6 & 8 & 11 & $8-14$ \\
Zilpzalp & 2 & 5 & 8 & $4-14$ \\
Fitis & 3 & 8 & 8 & $5-13$ \\
\hline Amsel & 3 & 4 & 9 & $4-10$ \\
Goldammer & 2 & 7 & 10 & $4-11$ \\
Kohlmeise & 6 & 5 & 6 & $1-14$ \\
Blaumeise & 6 & 5 & 5 & $1-14$ \\
\hline Buchfink & $(1)$ & 5 & 9 & $4-13$ \\
Grünling & 8 & 4 & 8 & $1-14$ \\
Feldsperling & 6 & 4 & 11 & $1-14$ \\
Star & 7 & 4 & 5 & $1-14$ \\
\hline
\end{tabular}

Für die drei anderen Grasmückenarten ergeben sich daraus die in Tab. 14 angegebenen Zeiträume. Bei der Klappergrasmücke wurde abweichend von diesem Muster zusätzlich Dekade 4 ausgeschlossen, weil die Anfang Mai festgestellten Beringungszahlen trotz eines leichten Rückgangs noch auf starken Durchzug schließen lassen.

Die Nachtigall gehört zu den Langstreckenziehern und brütete (oft unregelmäßig) in sieben von 33 Hecken, mit durchschnittlich vier bis fünf Brutpaaren pro Jahr. Das Zeitfenster schließt beide Durchzugsgipfel der Adulten aus, womöglich aber eine große Anzahl von dispergierenden Jungvögeln im Juni und Juli mit ein.

Bei Amsel und Singdrossel ist der frühe Brutbeginn zu berücksichtigen. Dennoch erscheint bei der Amsel, nach der in Abb. 11 dargestellten Phänologie, eine Abtrennung der ersten drei Dekaden im April gerechtfertigt. Im Mai treten erste Jungvögel auf. Erst im Juni steigen die Beringungszahlen deutlich an. Bei beiden Arten wird durch zunehmende Fangzahlen juveniler Vögel deutlich, dass die zweite Brutphase erheblich erfolgreicher ist als die erste. Bei der Amsel findet sich von der 10. auf die 11. Dekade ein Anstieg um über $50 \%$, bei der 
Singdrossel eine Dekade später. Hierdurch wird das Ende des Betrachtungszeitraums definiert. Es ist dennoch möglich, dass einige Jungvögel dadurch nicht mehr erfasst werden, zumal beide Arten als regelmäßig mehrfach brütend beschrieben werden (GLUTZ VON BLOTZHEIM \& BAUER 1988b). Es wird dafür ein Zeitraum von acht Dekaden zur Erfassung von juvenilen Amseln und Singdrosseln abgedeckt, der weitgehend frei von dispergierenden Jungvögeln aus benachbarten Gebieten sein dürfte.

Das Rotkehlchen zeigt einen sehr auffälligen Gipfel im April. Ab Mai verbleibt dann der Fangerfolg an Adulten gleichbleibend niedrig. Die Fänge von Jungvögeln sind im Mai ebenfalls sehr gering und steigen erst Mitte Juni allmählich, Ende Juni und Mitte Juli nochmals stärker an. Rotkehlchen brüten zwei- und bei besten nahrungsökologischen Bedingungen über Schachtelbruten sogar bis zu dreimal im Jahr (GLUTZ VON BLOTZHEIM \& BAUER 1988a, GRAJETZKY 2000). Interessanterweise berichtet GRAJETZKY (1993), dass Rotkehlchen in den Hecken seines Untersuchungsgebietes keine Zweitbruten anfingen, obwohl solche auf einer Vergleichsfläche im Buchenwald regelmäßig festgestellt werden konnten. Darüber hinaus war der Prädationsdruck in den zumeist als Doppelknick ausgeprägten Heckenstrukturen so hoch, dass nur 0,8 Jungvögel pro Brutpaar flügge wurden. Unter diesen Umständen wäre eine Eingrenzung des Zeitfensters zwischen den Dekaden 4 bis maximal 8 sinnvoll. Da aber unter den hier betrachteten Hecken durchaus breite und waldartig ausgeprägte Standorte vertreten sind, wurde das Zeitfenster auf die 3.-10. Dekade festgelegt.

Das Fangmuster der Heckenbraunelle zeigt ein Abklingen der zunächst hohen Fangsummen in der ersten April-Dekade bis in den Mai hinein. Nach GLutz VON BLOTZHeIM \& BAuER (1985) „hält der Durchzug [in Mecklenburg] bis Mitte (Ende) April an“. Der Betrachtungszeitraum für die Indizierung des Bruterfolgs beginnt demzufolge im Mai, eine Dekade vor dem Auftreten der ersten Jungvögel. Obwohl bereits Anfang Juli in Süddeutschland und in der Reit deutliche Dispersionsbewegungen festzustellen sind (GLUTZ VON BlOtZHeim \& BAUER 1985, BERTHOLD et al. 1991), wurde hier der Betrachtungszeitraum bis in den August hinein (13. Dekade) gewählt. Zur 14. Dekade erfolgt eine Verdopplung der juvenilen Fänglinge. Der deutliche Anstieg von der 8. auf die 9. Dekade im Juni könnte auf erhöhten Bruterfolg in der zweiten Brutphase schließen lassen. In zwei Studien in England wurde ein deutlich höherer Bruterfolg für die späteren Bruten festgestellt (SNOW \& SNOW 1982, BIRKHEAD 1981 zit. in GLUTZ VON BLOTZHEIM \& BAUER 1985): 0,67 ausgeflogene Jungvögel pro Nest im April gegenüber 1,96 im Mai bis Juli. 
Gelbspötter und Sumpfrohrsänger erscheinen erst im Mai und weisen ein Durchzugsmaximum gegen Ende des Monats auf, so dass der Zeitraum zwischen Mitte Juni bis Mitte August betrachtet wurde. Adulte Sumpfrohrsänger werden auf der Fangstation in Galenbeck ab Mitte August nur noch spärlich festgestellt (BAIRLEIN 1997), und auch in dieser Studie weist ein Maximum der Altvögel in der letzten Juli-Dekade auf einen frühen Abzug hin. Die Ausdehnung des Betrachtungszeitraums ist ein Kompromiss aus den überlappenden Phänologien von Jung- und Altvögeln.

Beim Zilpzalp zeigt sich ein deutlicher Durchzugsgipfel im April. Da die Art im Untersuchungsgebiet (abgesehen von klimatisch extremen Standorten) regelmäßig versucht zwei Bruten durchzuführen, erstreckt sich der Betrachtungszeitraum bis Mitte August. Erhöhte Fangzahlen aufgrund von Migration werden sowohl in Süd- als auch in Norddeutschland erst ab Ende August bzw. Mitte September festgestellt (BerThold et al. 1991). Das Fangmaximum lag bei einer europaweit angelegten Studie zwischen 1994-1996 in der Reit (Norddeutschland) um Mitte September, am Bodensee um Ende September bis Anfang Oktober (BAIRLEIN 1997), so dass der gewählte Zeitraum gerechtfertigt erscheint.

Der Fitis ist Langstreckenzieher und führt in der Regel nur eine Jahresbrut durch (z.B. SCHÖNFELD 1984, BEZZEL 1993). In Abb. 10 weist Dekade 4 noch auf erhöhten Durchzug hin (Weibchen treffen bis zu drei Wochen später als die Männchen in den Brutgebieten ein) und in Dekade 14 macht sich der Durchzug von Jungvögeln deutlich bemerkbar. Es stellt sich die Frage, ob der Anstieg von Jungvögeln Anfang Juli bereits auf dismigrierende Jungvögel aus anderen Brutgebieten zurückzuführen ist. Nach BERTHOLD et al. (1991) liegen die Durchzugsmaxima bei einer Untersuchungsdauer von 10 Jahren für den Fitis sowohl auf der Mettnau (Süd-) als auch in der Reit (Norddeutschland) in der ersten Augustdekade. Auf der Greifswalder Oie, einer Ostsee-Insel, liegen maximale Fangzahlen zwischen dem 20. Juli - 8. August (BAIRLEIN 1997). Dieser Befund würde für einen kleineren Betrachtungszeitraum sprechen. Aus den Fangzahlen des Heckenprogramms ist jedoch erst mit der 14. Dekade ein sehr starker Anstieg der Jungvögel abgrenzbar. Ein möglicher Fehler durch bereits im Juli dismigrierende Vögel muss bei dieser Art in Kauf genommen werden.

Der Betrachtungszeitraum für die Goldammer umfasst die 4.-11. Dekade. Damit wurde das Maximum adulter Vögel im April und das der Jungvögel Ende Juli ausgeblendet. Im April können die einzelnen Reviere zwar schon festliegen, regional werden auch bereits Nester angelegt und Legebeginn registriert (BEZZEL 1993). Bei Kälteeinbrüchen (Temperatur unter $0^{\circ} \mathrm{C}$ ) erfolgt jedoch wieder Schwarmbildung und Vergesellschaftung mit Finken und 
Sperlingen (versch. Autoren in GLUTZ VON BLOTZHEIM \& BAUER 1997, eig. Beobachtungen). Auf diese Weise erklärtes Umherstreifen in sogenannten Winterschwärmen kann zu erhöhten Fangzahlen im April führen. Die Verdreifachung der Beringungen an Jungvögeln von Mitte Juli bis Ende Juli stimmt mit dem Ende der zweiten Brutzeit überein (LILLE 1996). Die Schlupftermine der ersten Jahresbrut sind in Norddeutschland weitgehend auf den Mai beschränkt, während der zweite Brutabschnitt von Juni bis Juli andauert (LILLE 1996), in Ausnahmen bis August und September (GLuTZ vOn BlotZHEIM \& BAUER 1997).

Der Status des Buchfinks in Mitteleuropa reicht vom Zugvogel im Norden, über Teilzieher bis zum reinen Standvogel in milden Regionen (BEzzEL 1993). In die hier betrachtete Periode von der 4. bis zur 13. Dekade können ein bis zwei Jahresbruten fallen, wobei letztere für die Art nur in ganz wenigen Fällen nachgewiesen worden sind (BERGMANN 1993). Der Anstieg von Fänglingen in der 14. Dekade kann auf einsetzende Dismigration zurückzuführen sein.

Kohlmeise, Blaumeise und Feldsperling siedeln innerhalb eines Radius weniger Kilometer um ihren Geburtsort und gelten als sehr standorttreu (BERNDT \& WINKEL 1987, WINKEL \& FRANTZEN 1989, WINKEL UND FRANTZEN 1991). Die Beringungsmuster bieten keinen Ansatz für die Abtrennung bestimmter Dekaden, so dass der gesamte Untersuchungszeitraum einbezogen wurde. Dieses gilt ebenso für Grünling und Star, die häufig in größeren Trupps, lokal und zeitlich konzentriert, in oder an Hecken erscheinen und für die der gesamte Fangzeitraum herangezogen wurde.

\subsubsection{Der relative Bruterfolg häufiger Brutvögel in Hecken}

Aus den Fangzahlen der artspezifisch festgelegten Zeiträume wurden Quotienten (I) aus dem Verhältnis Jungvögel/Altvögel (dj/nd) gebildet, die in Tab. 15 für die fünf Jahre des Heckenprogramms dargestellt sind.

Bei der Summierung der jährlich erfassten Jung- und Altvögel zeigte sich, dass 1996 das einzige Jahr im Untersuchungszeitraum war, in dem eine Abnahme des Jungvogelanteils zum Vorjahr vorlag. Sowohl 1995 als auch 1997 stieg das Verhältnis der Jungvögel zu den Altvögeln um jeweils etwa 25 \% zum Vorjahr an. 1998 war trotz der Zunahme des Vorjahres nochmals eine Erhöhung des Jungvogelanteils festzustellen, so dass der insgesamt höchste Wert von 1,82 für den relativen Bruterfolg erreicht wurde. 
Tab. 15: Indizierung des Bruterfolgs über das Verhältnis gefangener Jungvögel zu den Altvögeln (I), als Index aus dj/nd Fänglingen (unter Einschluss aller Erstfänge sowie der in Vorjahren beringten Vögel) und die prozentuale Veränderung dieses Index zum Vorjahr (\% VJ). MW bezeichnet den Mittelwert des Index und unter $\mathbf{N}$ ist die Anzahl erfasster Individuen über alle Untersuchungsjahre angegeben.

\begin{tabular}{|c|c|c|c|c|c|c|c|c|c|c|c|}
\hline \multirow[b]{2}{*}{ Art } & \multirow{2}{*}{$\begin{array}{c}1994 \\
\text { I }\end{array}$} & \multicolumn{2}{|c|}{1995} & \multicolumn{2}{|c|}{1996} & \multicolumn{2}{|c|}{1997} & \multicolumn{2}{|c|}{1998} & \multirow[b]{2}{*}{ MW } & \multirow[b]{2}{*}{$\mathbf{N}$} \\
\hline & & I & $\% \mathbf{V J}$ & I & $\% \mathbf{V J}$ & I & $\% \mathbf{V J}$ & I & $\% \mathbf{V J}$ & & \\
\hline Mönchsgrasmücke & 1,32 & 1,14 & -14 & 0,72 & -37 & 1,13 & 57 & 1,43 & 27 & 1,15 & 1759 \\
\hline Gartengrasmücke & 0,58 & 0,43 & -26 & 0,29 & -33 & 0,58 & 100 & 0,81 & 40 & 0,49 & 508 \\
\hline Dorngrasmücke & 0,6 & 1,11 & 85 & 0,66 & -41 & 0,94 & 42 & 0,75 & -20 & 0,82 & 450 \\
\hline Klappergrasmücke & 0,29 & 1 & 245 & 0,19 & -81 & 0,58 & 205 & 0,52 & -10 & 0,46 & 218 \\
\hline Nachtigall & & 2,75 & & 1,4 & -49 & 3,5 & 150 & 1,2 & -66 & 1,66 & 133 \\
\hline Singdrossel & 1,42 & 0,85 & -40 & 1 & 18 & 0,36 & -64 & 1,39 & 286 & 0,92 & 229 \\
\hline Rotkehlchen & 1,65 & 1,08 & -35 & 0,54 & -50 & 1,73 & 220 & 1,53 & -12 & 1,2 & 361 \\
\hline Heckenbraunelle & 1,3 & 1,43 & 10 & 0,9 & -37 & 1,19 & 32 & 2,12 & 78 & 1,37 & 644 \\
\hline Sumpfrohrsänger & 0,8 & 0,85 & 6 & 0,42 & -51 & 0,48 & 14 & 0,59 & 23 & 0,55 & 352 \\
\hline Gelbspötter & 0,75 & 0,7 & -7 & 0,48 & -31 & 0,75 & 56 & 1,58 & 111 & 0,78 & 130 \\
\hline Zilpzalp & 2,29 & 4,64 & 103 & 3,06 & -34 & 3,5 & 14 & 3,24 & -7 & 3,34 & 2594 \\
\hline Fitis & 1,65 & 1,97 & 19 & 1,79 & -9 & 1,55 & -13 & 1,49 & -4 & 1,67 & 715 \\
\hline Amsel & 0,34 & 0,77 & 126 & 0,57 & -26 & 0,54 & -5 & 0,7 & 30 & 0,58 & 524 \\
\hline Goldammer & 0,24 & 0,85 & 254 & 0,74 & -13 & 1,31 & 77 & 2,05 & 56 & 1,1 & 560 \\
\hline Kohlmeise & 2,81 & 2,87 & 2 & 2,13 & -26 & 2,9 & 36 & 3,21 & 11 & 2,7 & 2436 \\
\hline Blaumeise & 3,17 & 2,67 & -16 & 3,56 & 33 & 3,15 & -12 & 5,06 & 61 & 2,88 & 1054 \\
\hline Buchfink & 0,22 & 0,59 & 168 & 0,63 & 7 & 1,08 & 71 & 0,35 & -68 & 0,55 & 258 \\
\hline Feldsperling & 0,44 & 2,43 & 452 & 0,57 & -77 & 0,33 & -42 & 0,23 & -30 & 0,6 & 463 \\
\hline Summe aller Arten & 1,34 & 1,66 & 24 & 1,26 & -24 & 1,57 & 25 & 1,82 & 16 & 1,54 & 13388 \\
\hline
\end{tabular}

Aus Tab. 15 geht hervor, dass 1996 für die meisten Grasmücken-Arten (Ausnahme Dorngrasmücke) die geringste Jungvogel/Altvogel Proportion errechnet wurde. Weil auch Rotkehlchen, Heckenbraunelle, Sumpfrohrsänger, Gelbspötter und Kohlmeise in diesem Jahr den geringsten Anteil von Jungvögeln aufwiesen, hatten 1996 insgesamt acht Arten ihren geringsten relativen Bruterfolg. 1995 erreichten dagegen mit Dorn- und Klappergrasmücke, Amsel, Sumpfrohrsänger, Zilpzalp, Fitis und Feldsperling sieben Arten ihre jeweils höchste Jungvogel-Altvogel Proportion. In 1995 hatten die meisten Arten den höchsten relativen Bruterfolg über den Untersuchungszeitraum, obwohl der absolut höchste Index-Wert 1998 erreicht wurde. In 1996 hatten dagegen die meisten Arten ihren niedrigsten I-Wert, so dass 1996 auch bei der Betrachtung einzelner Arten als das Jahr mit dem schlechtesten Bruterfolg gelten muss. 1997 stieg der 'relative Bruterfolg' insgesamt wieder an. Nur Amsel und Singdrossel, Feldsperling und Fitis zeigten noch eine negative Entwicklung im Vergleich zum Vorjahr. Für den Fitis war von 1995 bis 1998 ein steter Rückgang des Jungvogelanteils festzustellen. 1998 zeigten mit Mönchs- und Gartengrasmücke, Heckenbraunelle, Gelbspötter, Goldammer und Kohlmeise sechs Arten ein Maximum. Nachtigall, Fitis und Feldsperling dagegen hatten ihren geringsten 'relativen Bruterfolg'. Nach 1995 tritt 1998 somit ein weiteres Jahr auf, in dem für viele Arten der höchste Bruterfolgs-Index erreicht 
wird, wobei auch drei Arten mit ihren niedrigsten I-Werten dagegen stehen. Mit der Summierung des 'relativen Bruterfolgs' über die 16 häufigsten Heckenvögel wird die Dynamik der Reproduktivität über den Untersuchungszeitraum sichtbar (Abb. 12).

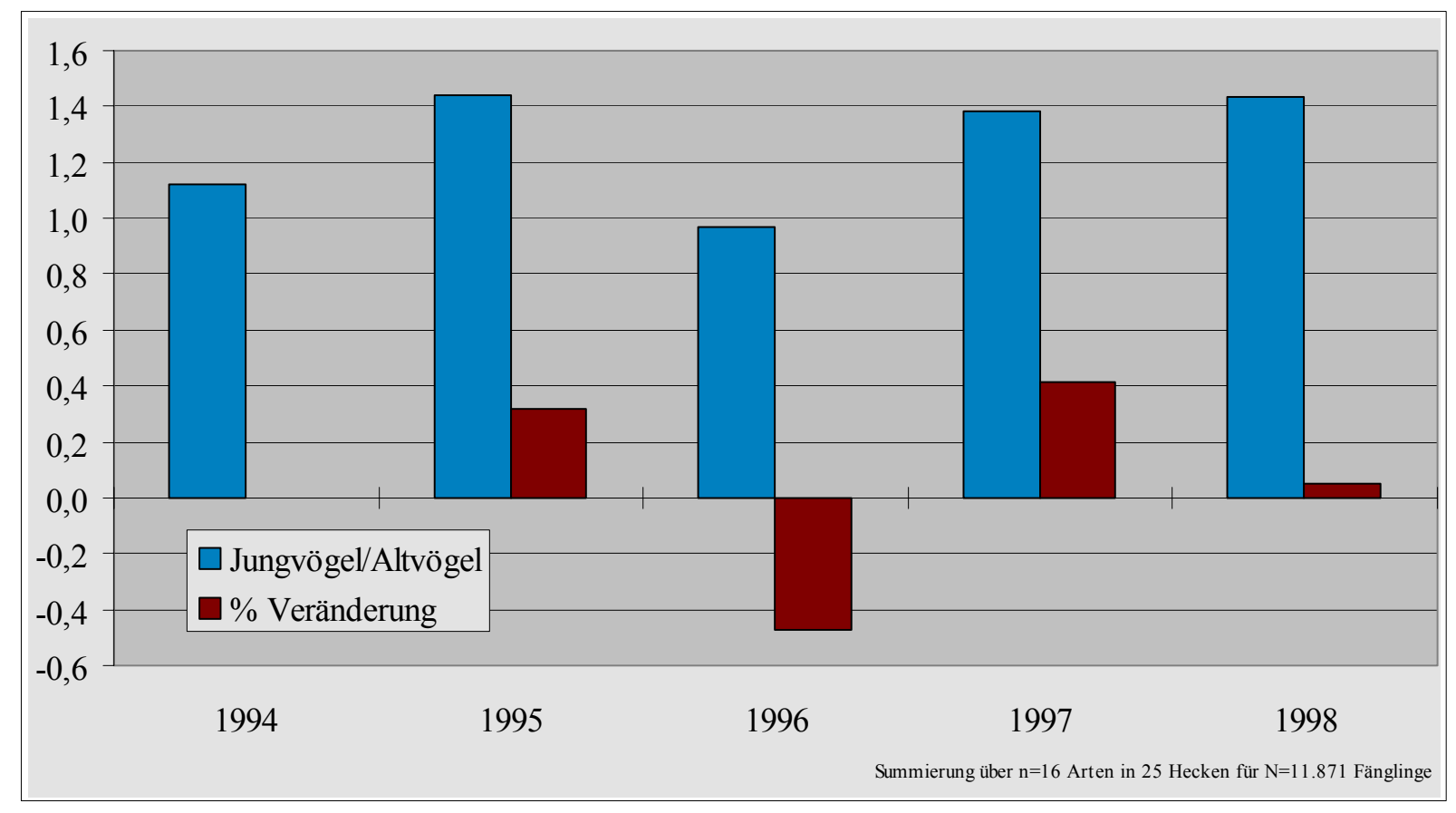

Abb. 12: Der „relative Bruterfolg“ aus dem Verhältnis der Jung- zu den Altvögeln als Summierung über 16 Arten mit N=11.871 Fänglingen aus 25 Hecken und die jeweilige prozentuale Veränderung zum Vorjahr (Arten wie in Tab. 15, ohne Feldsperling und Blaumeise).

Der geringe Bruterfolg vieler Arten 1996 wird deutlich. Die Jahre 1995, 1997 und 1998 zeigen ein einheitliches Niveau von etwa 1,4 Jungvögeln auf einen Altvogel. Anhand der Bruterfolgs-Indizes ist nur ein Vergleich über die Jahre oder zwischen verschiedenen Gebieten, aber keine qualifizierende Aussage darüber möglich, ob der Bruterfolg einer Art ausreichend ist, um die Mortalität der Population auszugleichen.

In Abb. 12 ist der Feldsperling ausgenommen, weil diese Art sehr unstet gefangen wird und infolgedessen sprunghafte Bestandszahlen zeigen kann, die nicht den tatsächlichen Brutbestand widerspiegeln. Bei der Blaumeise ist nicht auszuschließen, dass eine unbestimmte Anzahl von Nestlingen zu den diesjährigen Fänglingen gezählt worden ist, so dass auch diese Art hier ausgeschlossen wurde.

Die Darstellung einer über die Arten summierenden Graphik kann zum optischen Vergleich mit den artspezifischen Mustern einzelner Heckenbrüter herangezogen werden (Abb. 13). Dadurch bietet sich eine einfache Möglichkeit, festzustellen inwiefern einzelne Arten Abweichungen von dem allgemeinen Verlauf des 'relativen Bruterfolgs' zeigen. 

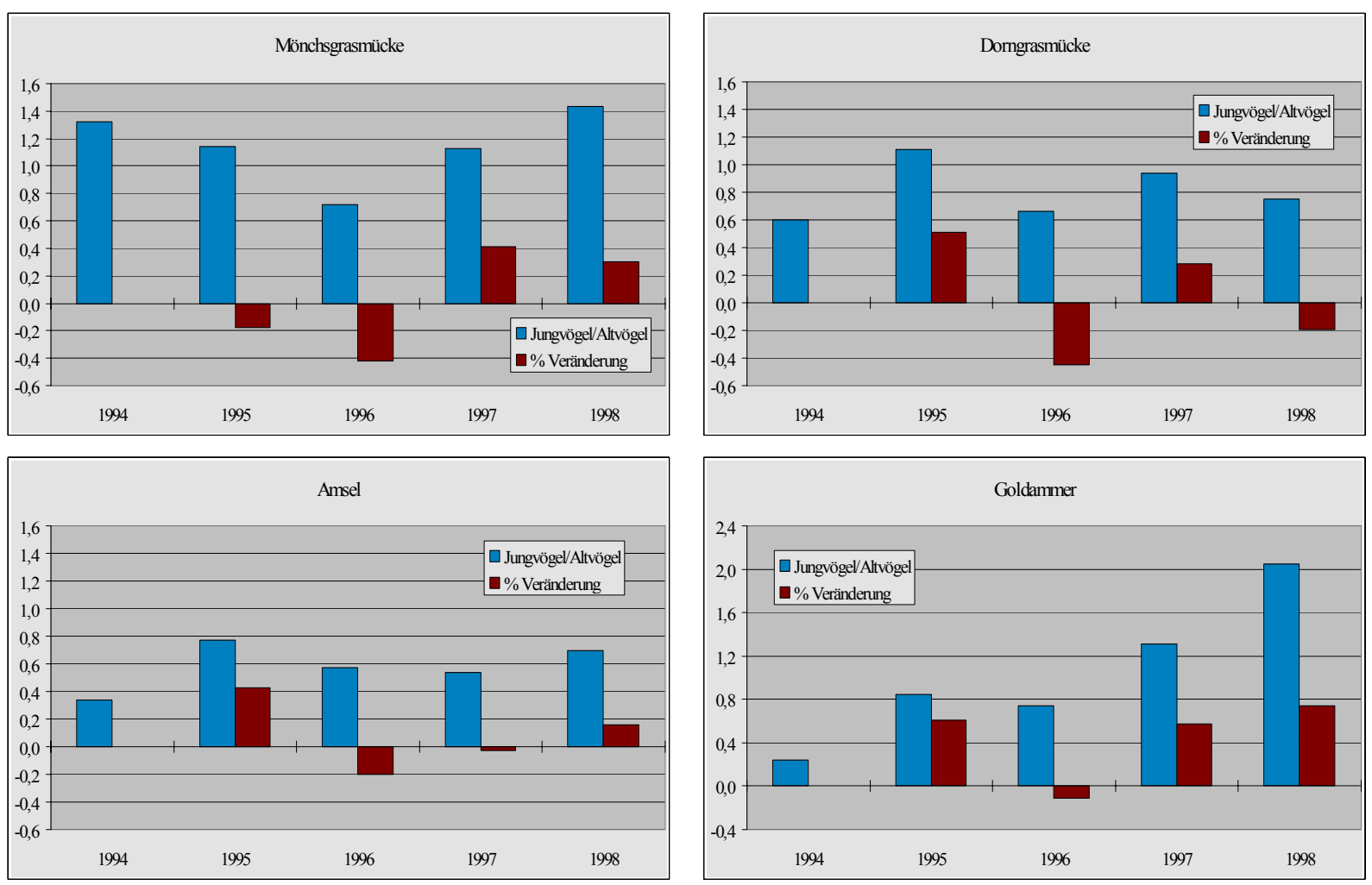

Abb. 13: Der „relative Bruterfolg“ aus dem Verhältnis der Jung- zu den Altvögeln für Mönchs- und Dorngrasmücke, Amsel und Goldammer aus 25 Hecken und die jeweilige prozentuale Veränderung zum Vorjahr, für $\mathbf{N}$ der Arten siehe Tab. 15.

E: Die in Abb. 13 dargestellten Arten hatten 1996 die größte negative Abweichung zum Vorjahr und stimmen darin mit der Summierung über alle Arten (Abb. 12) überein. Der 'relative Bruterfolg' von Mönchsgrasmücke und Goldammer verdoppelte sich zwischen 1996 bis 1998, während bei Amsel und Dorngrasmücke 1995 das erfolgreichste Brutjahr war. Nach der schlechten Brutsaison 1996 zeigte nur die Amsel 1997 nochmals einen Rückgang im 'relativen Bruterfolg', während 1998 die Dorngrasmücke die einzige Art war, die eine negative Abweichung zum Vorjahr aufwies.

D: Diese Beispiele zeigen, dass die brutökologischen Bedingungen in den Hecken für die Arten unterschiedliche Auswirkungen haben können: Durch Unterschiede im Beginn des Brutgeschäfts können Witterungsbedingungen je nach Vogelart in anderer Weise wirken, unterschiedliche Anpassungen an einen in Hecken sehr hohen Prädationsdruck erzeugen unterschiedliche Verluste und die Arten nutzen ein spezifisches Nahrungsangebot, das anthropogen bedingt sehr starken Veränderungen unterliegen kann.

Die Bedeutung der Indizierung des Bruterfolgs liegt darin, dass Habitate auf der Basis ihrer populationsökologischen Funktion verglichen und bewertet werden können und nicht allein durch die Anzahl anwesender Individuen, was einen erheblichen qualitativen Unterschied darstellt. Die Jahre 1995, 1997 und 1998 zeigten die höchsten Werte für den relativen 
Bruterfolg von Singvögeln in Hecken. 1996 wurden dagegen die meisten Erstfänge an adulten Vögeln gemacht (Tab. 11). Aufgrund der Anzahl registrierter Individuen würde man davon ausgehen, dass 1996 das erfolgreichste Jahr war, weil die Vogeldichte am höchsten war. Die Untersuchung des relativen Bruterfolgs zeigte aber, dass die Produktivität der höheren Anzahl von Altvögeln 1996 nicht in proportional zu den Vergleichsjahren war. Die Anzahl von Jungvögeln war 1996 geringer als 1997 und 1998 (Tab. 49). An diesem Beispiel zeigt sich die Bedeutung und die Notwendigkeit eines integrierten Erfassens von Vogelpopulationen, wenn es um die Bewertung von Bestandsverläufen oder die Beurteilung von Habitaten geht.

\subsubsection{Beeinträchtigung des Bruterfolgs}

In einer Rubrik 'Beeinträchtigungen' auf dem Heckenerfassungsbogen wurden Störungen an Hecken erfasst. In Tab. 16 sind Einflüsse verschiedener Faktoren, die negativ auf den Bruterfolg einwirken können, dargestellt. Die Zusammenstellung basiert auf nicht quantifizierten Beobachtungen im Rahmen des Heckenprogramms.

Tab. 16: Einflüsse von Faktoren, die den Bruterfolg beeinträchtigen können.

\begin{tabular}{|c|l|l|}
\hline Faktor & \multicolumn{1}{|c|}{ Negativer Einfluss auf den Bruterfolg durch: } & \multicolumn{1}{c|}{ Folge } \\
\hline Prädation & $\begin{array}{l}\text { Heckenstruktur und hohe Räuberdichte } \\
\text { bei hohem 'Angebot' von Nestern }\end{array}$ & $\begin{array}{l}\text { Brutverlust, geringer } \\
\text { Ausfliegeerfolg }\end{array}$ \\
\hline Witterung & Kälte, Regen, Sturm, Hagel, Trockenheit, etc. & Brutverlust (und weitere indirekte Folgen) \\
\hline Nahrung & $\begin{array}{l}\text { Artenarme Hecken, Überalterung, Nutzung, } \\
\text { Pflege, Landwirtschaft, etc. }\end{array}$ & $\begin{array}{l}\text { geringer Ausfliegeerfolg, schwache } \\
\text { Jungvögel, dadurch geringes Recruitment }\end{array}$ \\
\hline Störungen & $\begin{array}{l}\text { Spaziergänger (Hunde), jagdliche Nutzung, } \\
\text { Bewirtschaftung }\end{array}$ & $\begin{array}{l}\text { Brutverlust, Brutunterbrechung, } \\
\text { geringes Recruitment }\end{array}$ \\
\hline Bewirtschaftung & $\begin{array}{l}\text { Ernte, Mahd, Landschaftspflege } \\
\text { (z.B. Ausmähen von Randstreifen) }\end{array}$ & $\begin{array}{l}\text { Brutverlust, Nahrungsmangel, } \\
\text { geringes Recruitment }\end{array}$ \\
\hline
\end{tabular}

Von den fünf genannten Faktoren, die den Bruterfolg von Heckenvögeln beeinträchtigen können, führen vier in der Regel zu totalem Brutverlust. Als weitere Ursachen, die zur Verminderung des Bruterfolgs beitragen, wären noch Parasitenbefall an Nestlingen und die Parasitierung von Nestern durch den Kuckuck zu nennen.

Mit der Dokumentation des 'relativen Bruterfolgs' über die Zeit können artübergreifend Jahre mit relativ hohem und niedrigem Reproduktionsniveau ermittelt werden. Ist der 'relative Bruterfolg' über die Arten gleichgerichtet, wäre zu vermuten, dass in erster Linie unspezifische Faktoren auf den Bruterfolg wirken (z.B. Witterung, allgemein hohe Räuberdichten). Wie die Beispiele in Abb. 13 zeigen, ist das nur teilweise der Fall (allgemein geringerer 'relativer Bruterfolg' 1996 im Vergleich zum Vorjahr). Aber der 'relative Bruterfolg' hängt 
auch von artspezifisch wirkenden Faktoren ab. Dieses zeigt sich in Abweichungen der Bruterfolge einzelner Arten, z.B. Mönchsgrasmücke 1995, Amsel 1997 oder Dorngrasmücke 1998. Auf der Ebene einzelner Arten können lebensraumspezifische Einflüsse auf den Bruterfolg deutlich werden. Das wäre anhand von Reproduktionsraten in strukturell verschiedenen Habitaten vergleichbar. Für eine derartige Analyse reicht jedoch die Dichte der Daten dieser Untersuchung nicht aus. Auch ein detaillierter Vergleich der unterschiedlichen Höhe des 'relativen Bruterfolgs' zwischen den Arten ist problematisch, weil die Arten starke Abweichungen in ihrer Reproduktionsleistung (einige Arten brüten nur einmal, während andere zwei reguläre Bruten pro Jahr durchführen können) und in den spezifischen Fangmustern zeigen. Eine hohe Anzahl durchziehender Individuen kann die Ergebnisse beeinflussen, bei einigen Arten nutzen vor allem nichtterritoriale Individuen die Hecken (Rotkehlchen), bei anderen Arten sind vor allem die Brutvögel an die Hecke als Neststandort gebunden, während die Nahrungssuche außerhalb der Hecken stattfindet (vgl. BLAB 1993), so dass ein zwischenartlicher Vergleich eines Reproduktionsindexes ökologisch nicht sinnvoll erscheint.

\subsection{Wiederfänge}

Als Wiederfänge (W) sind bereits beringte Vögel definiert, die im laufenden Kalenderjahr nochmals in der Hecke gefangen wurden (Tab. 17).

Diese Tiere sind mit hoher Wahrscheinlichkeit territorial und repräsentieren einen Teil des Brutbestandes der Art. Von den Programmvögeln wurden im Verlauf der Untersuchung während der Brutzeit bei 33 Arten Wiederfänge gemacht. Von vielen Arten konnten sowohl Alt- als auch Jungvögel wiedergefangen werden. In Tab. 17 ist nur die Anzahl der Altvögel dargestellt, weil nur diese als Vergleichsbasis zu Ergebnissen der Kartierungen dienen können.

Die höchsten Anzahlen von Wiederfängen hatten Zilpzalp, Mönchsgrasmücke und Heckenbraunelle. Über alle Programmarten wurden durchschnittlich 14,8 \% der Erstfänge wiedergefangen. Von den 20 Arten mit mehr als 20 Wiederfängen hatte die Heckenbraunelle mit $28 \%$ die höchste Wiederfangrate. Sehr hohe Raten wurden auch bei den Höhlenbrütern erreicht. Die vier Meisenarten Sumpf-, Weiden-, Blau- und Kohlmeise sowie Kleiber und Trauerschnäpper zeigten über $20 \%$ Wiederfangrate. Neben der Schwanzmeise lagen außerdem Amsel und Neuntöter über $20 \%$. 
Tab. 17: Wiederfänge (W) adulter Programmvögel und deren Anteil am Gesamtfang der Art (\%), N=Gesamtfang der Altvögel in 23 Hecken.

\begin{tabular}{|c|c|c|c|c|c|c|c|}
\hline Art & $\mathbf{W}$ & $\%$ & $\mathbf{N}$ & Art & $\mathbf{W}$ & $\%$ & $\mathbf{N}$ \\
\hline 1 Zilpzalp & 234 & 17,6 & 1331 & 18 Gelbspötter & 23 & 13,1 & 175 \\
\hline 2 Mönchsgrasmücke & 170 & 12,7 & 1341 & 19 Neuntöter & 22 & 25,3 & 87 \\
\hline 3 Heckenbraunelle & 164 & 28,0 & 586 & 20 Zaunkönig & 22 & 28,6 & 77 \\
\hline 4 Kohlmeise & 142 & 21,0 & 675 & 21 Weidenmeise & 18 & 28,6 & 63 \\
\hline 5 Amsel & 129 & 20,9 & 616 & 22 Sumpfmeise & 12 & 40,0 & 30 \\
\hline 6 Gartengrasmücke & 121 & 17,6 & 687 & 23 Gimpel & 10 & 15,4 & 65 \\
\hline 7 Grünling & 98 & 15,7 & 625 & 24 Schwanzmeise & 9 & 23,1 & 39 \\
\hline 8 Dorngrasmücke & 80 & 15,4 & 521 & 25 Feldsperling & 8 & 2,5 & 327 \\
\hline 9 Goldammer & 67 & 16,6 & 404 & 26 Kleiber & 7 & 50,0 & 14 \\
\hline 10 Blaumeise & 62 & 24,9 & 249 & 27 Trauerschnäpper & 7 & 21,2 & 33 \\
\hline 11 Rotkehlchen & 52 & 8,2 & 632 & 28 Gartenbaumläufer & 2 & 11,8 & 17 \\
\hline 12 Sumpfrohrsänger & 48 & 11,8 & 408 & 29 Feldschwirl & 2 & 7,4 & 27 \\
\hline 13 Klappergrasmücke & 36 & 11,6 & 310 & 30 Star & 2 & 0,0 & 383 \\
\hline 14 Fitis & 33 & 6,3 & 528 & 31 Kernbeißer & 1 & 2,4 & 41 \\
\hline 15 Nachtigall & 27 & 18,9 & 143 & 32 Girlitz & 1 & 2,2 & 46 \\
\hline 16 Buchfink & 26 & 7,1 & 365 & 33 Gartenrotschwanz & 1 & 1,8 & 57 \\
\hline 17 Singdrossel & 25 & 12,2 & 205 & & & & \\
\hline
\end{tabular}

Bei 6 Programmarten (Tab. 17) und 10 Nicht-Programmarten gab es weniger als 10\% Wiederfänge: Bachstelze, Haussperling (je 6), Waldbaumläufer, Teichrohrsänger (3), Kleinspecht (2), Blaukehlchen, Sommergoldhähnchen, Rohrammer, Hausrotschwanz und Eichelhäher (1).

\subsubsection{Entwicklung der Wiederfangraten über die Jahre}

Für Arten, die relativ häufig wiedergefangen wurden, ist in Tab. 18 die Entwicklung der Wiederfänge über die Dauer des Heckenprogramms dargestellt.

E: Die Anzahl von Wiederfängen konnte bei manchen Arten beträchtlich schwanken (z.B. Zilpzalp, Kohlmeise), blieb aber bei anderen Arten über die Jahre auf einem relativ konstanten Niveau (z.B. Heckenbraunelle und Mönchsgrasmücke zwischen 1995 und 1998).

Die Differenz der prozentual erfassten Wiederfänge zum Vorjahr (in Tab. 18 unter D) kann als $\mathrm{Maß}$ für die Brutbestandsdynamik herangezogen werden. Die stärksten Rückgänge an Wiederfängen fanden sich ausnahmslos in den Jahren 1997 und 1998, während in 1995 und 1996 die höchsten Zunahmen an Wiederfängen registriert werden konnten. Nur drei Arten, Zilpzalp (+26), Blau- (+3) und Weidenmeise (+6) hatten 1997 den höchsten Zuwachs.

D: Dieses allgemeine Fangmuster steht in Übereinstimmung mit den Fangergebnissen adulter Vögel in Tab. 11. In beiden Darstellungen wird deutlich, dass die Jahre 1995 und 1996 für die meisten Arten positive Bestandsentwicklungen aufwiesen. 1997 war geprägt von Bestands- 
rückgängen bei 15 von 22 Arten in Tab. 11 und für ebenso viele von 20 Arten in Tab. 18. Die einzigen vier Arten deren Wiederfänge 1998 zunahmen, Heckenbraunelle, Rotkehlchen, Nachtigall und Goldammer, zeigten in dem Jahr auch bei den Erstfängen positive Tendenz. Unter den vergleichsweise stärker abnehmenden Wiederfängen (12 Arten mit $\mathrm{D}<-1)$ fanden sich auch die sechs Arten, deren Erstfänge rückläufig waren. Gelbspötter (-5), Zilpzalp (-23) und Fitis (-5) hatten 1998 ihre niedrigsten Anzahlen von Wiederfängen und weisen gleichzeitig bei den Erstfängen sehr hohe Fangzahlen auf (Zilpzalp und Fitis erreichen mit 147 bzw. 61 beringten Vögeln auf 100 Fangtage ihre Maximalwerte).

Tab. 18: Wiederfänge $(W)$ von adulten Programmarten (mit $W>20)$ und deren Anteil an Erstfängen (\%) zwischen 1994 bis 1998 sowie die Differenz zur Summe der Wiederfänge vom Vorjahr (D). W (ges) =Anzahl aller Wiederfänge, \% $(\mathrm{MW})=$ Mittelwert der jährlichen Anteile und N=Gesamtfang nd/F der Art.

\begin{tabular}{|c|c|c|c|c|c|c|c|c|c|c|c|c|c|c|c|c|c|}
\hline \multirow[b]{2}{*}{ Art } & \multicolumn{2}{|c|}{1994} & \multicolumn{3}{|c|}{1995} & \multicolumn{3}{|c|}{1996} & \multicolumn{3}{|c|}{1997} & \multicolumn{3}{|c|}{1998} & \multirow[b]{2}{*}{ W (ges) } & \multirow[b]{2}{*}{$\%(\mathrm{MW})$} & \multirow[b]{2}{*}{$\mathbf{N}$} \\
\hline & $\mathbf{W}$ & $\%$ & $\mathbf{W}$ & $\%$ & D & $\mathbf{W}$ & $\%$ & D & $\mathbf{W}$ & $\%$ & D & W & $\%$ & D & & & \\
\hline Zaunkönig & 3 & 42,9 & 5 & 27,8 & 2 & 6 & 21,4 & 1 & 5 & 50 & -1 & 3 & 21,4 & -2 & 22 & 28,6 & 77 \\
\hline Heckenbraunelle & 15 & 23,1 & 33 & 31,1 & 18 & 47 & 28 & 14 & 33 & 31,4 & -14 & 36 & 25,4 & 3 & 164 & 28 & 586 \\
\hline Rotkehlchen & 4 & 9,5 & 17 & 14,2 & 13 & 18 & 9,1 & 1 & 5 & 3,8 & -13 & 8 & 5,6 & 3 & 52 & 8,2 & 632 \\
\hline Nachtigall & 0 & & 3 & 27,3 & 3 & 11 & 33,3 & 8 & 4 & 15,4 & -7 & 9 & 12,5 & 5 & 27 & 18,9 & 143 \\
\hline Amsel & 21 & 18,1 & 35 & 29,4 & 14 & 38 & 21,1 & 3 & 18 & 17,3 & -20 & 17 & 17,5 & -1 & 129 & 20,9 & 616 \\
\hline Singdrossel & 2 & 11,1 & 4 & 11,4 & 2 & 7 & 10,1 & 3 & 10 & 19,6 & 3 & 2 & 6,3 & -8 & 25 & 12,2 & 205 \\
\hline Mönchsgrasmücke & 18 & 3,8 & 33 & 13,4 & 15 & 42 & 15,3 & 9 & 39 & 14,2 & -3 & 38 & 11,4 & -1 & 170 & 12,7 & 1341 \\
\hline Gartengrasmücke & 22 & 21,8 & 26 & 24,3 & 4 & 27 & 15,9 & 1 & 29 & 18,4 & 2 & 17 & 11,3 & -12 & 121 & 17,6 & 687 \\
\hline Klappergrasmücke & 7 & 15,2 & 7 & 11,5 & 0 & 9 & 11,7 & 2 & 8 & 14,3 & -1 & 5 & 7,1 & -3 & 36 & 11,6 & 310 \\
\hline Dorngrasmücke & 9 & 28,1 & 11 & 12,5 & 2 & 27 & 20,3 & 16 & 17 & 14,2 & -10 & 16 & 10,8 & -1 & 80 & 15,4 & 521 \\
\hline Sumpfrohrsänger & 2 & 15,4 & 9 & 17,3 & 7 & 17 & 14,8 & 8 & 15 & 11,6 & -2 & 5 & 5,1 & -10 & 48 & 11,8 & 408 \\
\hline Gelbspötter & 1 & 14,3 & 4 & 13,8 & 3 & 7 & 14,9 & 3 & 8 & 21,1 & 1 & 3 & 5,6 & -5 & 23 & 13,1 & 175 \\
\hline Zilpzalp & 12 & 11,1 & 37 & 19,5 & 25 & 52 & 16,3 & 15 & 78 & 26,7 & 26 & 55 & 13,1 & -23 & 234 & 17,6 & 1331 \\
\hline Fitis & 4 & 7,7 & 7 & 6 & 3 & 11 & 9,2 & 4 & 8 & 7,4 & -3 & 3 & 2,3 & -5 & 33 & 6,3 & 528 \\
\hline Kohlmeise & 20 & 22,5 & 17 & 17,2 & -3 & 50 & 24,3 & 33 & 34 & 23,6 & -16 & 21 & 15,3 & -13 & 142 & 21 & 675 \\
\hline Blaumeise & 14 & 40 & 15 & 28,8 & 1 & 12 & 17,9 & -3 & 15 & 26,3 & 3 & 6 & 15,8 & -9 & 62 & 24,9 & 249 \\
\hline Neun & 1 & 14,3 & 5 & 33,3 & 4 & 9 & 28,1 & 4 & 5 & 25 & -4 & 2 & 15,4 & -3 & 22 & 25,3 & 87 \\
\hline Grün & 57 & 24,6 & 20 & 17,5 & -37 & 16 & 12,6 & -4 & 4 & 4,7 & -12 & 1 & 1,5 & -3 & 98 & 15,7 & 625 \\
\hline Buchfink & 9 & 14,3 & 2 & 3 & -7 & 8 & 6,7 & 6 & 4 & 7,8 & -4 & 3 & 4,6 & -1 & 26 & 7,1 & 365 \\
\hline Goldammer & 7 & 13 & 9 & 11,4 & 2 & 31 & 26,1 & 22 & 8 & 14 & -23 & 12 & 12,6 & 4 & 67 & 16,6 & 404 \\
\hline
\end{tabular}

Neben Gelbspötter und Fitis sind Gartengrasmücke und Sumpfrohrsänger zwei weitere Arten, die zu den Langstreckenziehern gehören und deren Wiederfänge 1998 stark zurück gingen. Weil auch Neuntöter (15,3\% bei $\mathrm{MW}=21)$ und Dorngrasmücke (mit 10,8 \% bei $\mathrm{MW}=15,4$ der niedrigste relative Wert) eine sehr geringe Anzahl von Wiederfängen aufwiesen, lässt sich feststellen, dass vor allem Zugvögel 1998 von den Rückgängen betroffen waren. Als Ursachen kommen Verluste im Winterquartier und auf dem Zug, Zugstau und späteres Eintreffen in den Brutgebieten mit geringerer Chance auf Wiederfang in Frage. Ein allgemeiner Rückgang der Arten kann nicht belegt werden, nur Sumpfrohrsänger und Neuntöter zeigten 1998 auch bei den adulten Erstfängen eine negative Populationsentwicklung (Tab. 11).

Ein hoher Prozentsatz unter den Erstfängen, die nicht als Wiederfang erschienen, sind wahrscheinlich Durchzügler, die aber den Anteil von Wiederfängen beeinflussen können. Als 
Beispiel sei der Sumpfrohrsänger angeführt: 1994 wurden nur zwei Individuen wieder gefangen und entsprachen 15,4\% aller Erstberingten des Jahres, 1996 wurden mit 17 Wiederfängen nur 14,8 \% aller Fänglinge erfasst.

Der Rückgang von Wiederfängen über den Untersuchungszeitraum könnte u. U. auch auf einen methodischen Fehler zurückzuführen sein, wenn einige Kontrollfänge (in Vorjahren bereits beringte Individuen) ebenso Revierinhaber sind, aber nicht zum zweiten mal in der Saison gefangen werden und somit nicht als Wiederfang registriert werden. Das könnte der Fall sein, wenn ältere Individuen ('mit Netzerfahrung') aufgrund ihrer Ortskenntnis in der Lage sind, Netzstandorte als solche zu erkennen und diese (oder damit einhergehende menschliche Aktivität) meiden (LOVEJOY 1974). MURRAY (1997) ging der Vermutung von Beringern nach, dass ein Rückgang der Fangzahlen einer amerikanischen Drosselart (Wood Thrush, Hylocichla mustelina) auf der Meidung von Netzen basiere. Der Autor konnte aber weder die Meidung von Netzen noch den Rückgang der Art bestätigen. Dennoch weisen viele Beobachtungen (eigene und Berichte von Mitarbeitern des Heckenprogramms) darauf hin, dass territoriale Individuen eine sehr präzise Kenntnis der Strukturen in ihrem Revier haben und Netze vor allem in unmittelbarer Nähe ihres Brutplatzes umfliegen.

\subsubsection{Wann treffen die Revierinhaber in den Brutgebieten ein?}

Wiedergefangene Vögel deuten auf eine Ortsbindung dieser Individuen hin. Somit lässt sich für die Brutzeit der Schluss ziehen, dass Wiederfänglinge Revierinhaber sind.

Zur Indizierung des Bruterfolgs über das Verhältnis von Jung- zu Altvögeln war es notwendig, Zeiten hoher Durchzugsaktivität in den Hecken für die Berechnung des Index auszuschließen. Im April und Mai sind davon ausschließlich Altvögel betroffen. Unter diesen können aber auch Individuen sein, die als Revierinhaber über die Brutperiode in den Hecken verbleiben. Mit der Aufnahme von Wiederfängen ergibt sich nun die Möglichkeit, erstens das Ankunftsdatum von zukünftigen Revierinhabern festzustellen (sofern der Beginn des Fangzeitraums das Eintreffen der Art erfasst) und zweitens allgemeine Fangmuster von Brutvögeln (unter der Annahme, dass wiedergefangene Vögel territorial und somit Revierinhaber in der untersuchten Hecke sind) mit denen aller Erstfänge zu vergleichen. Dieses kann aufgrund des Datenmaterials nur exemplarisch getan werden.

E: Das in Abb. 14 dargestellte Diagramm von Erstfängen, die später nochmals gefangen werden konnten, ist beim Zilpzalp weitgehend identisch mit dem Fangmuster, das bei allen Erstfängen der Art festgestellt wurde (vgl. Abb. 10). 


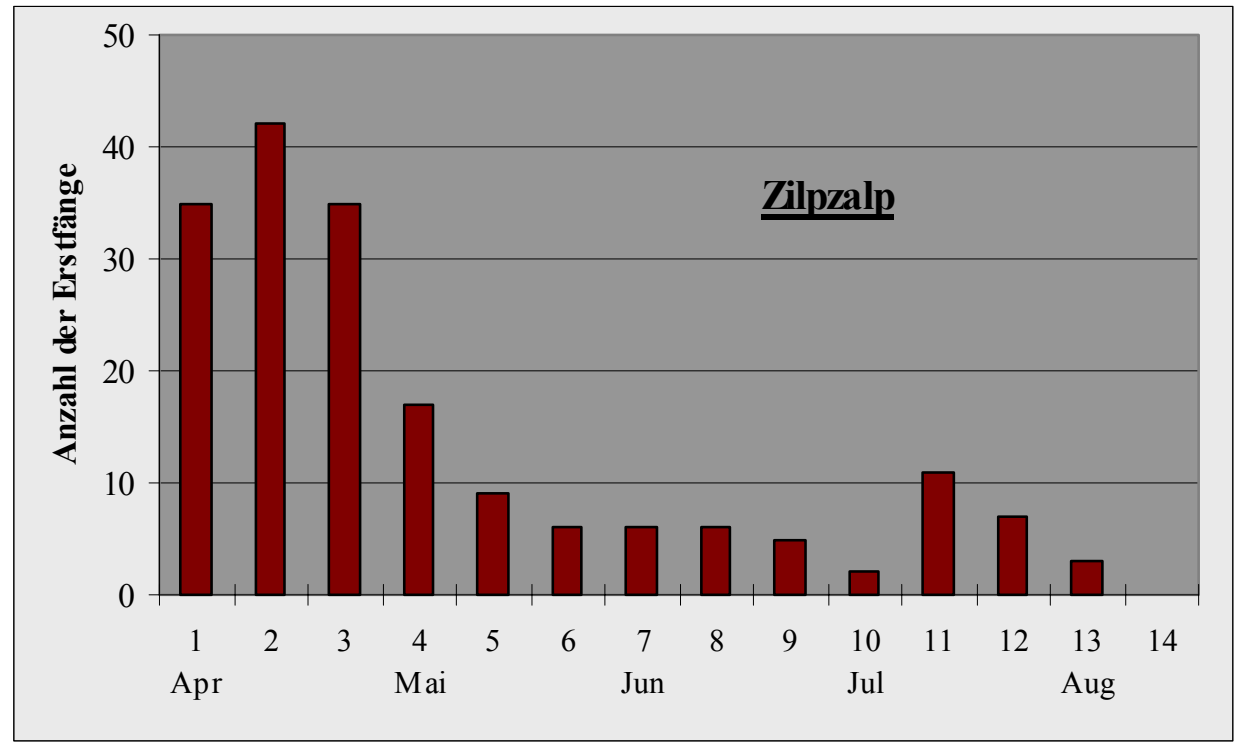

Abb. 14: Fangzeitpunkte von Erstfängen des Zilpzalp, die später als Wiederfänge registriert werden konnten (23 Hecken zwischen 1994-1998, $n=184)$.

Die Verteilung der Wiederfänge weicht von einer erwarteten Verteilung nach der Anzahl der Erstfänge nicht $\mathrm{ab}\left(\mathrm{Chi}^{2}=18,92, \mathrm{FG}=13\right.$, mit $\mathrm{p}=0,13$, n.s. $)$.

\subsubsection{Interpretation der Fangmuster}

Für die Interpretation von Fangmustern ist die Ankunft von späteren Revierinhabern von Bedeutung. So ist es zum Beispiel für die Interpretation von frühen Begehungen bei Revierkartierungen von Interesse zu wissen, ob die ersten Sänger einer ziehenden Art die ersten Revierinhaber sind oder ob diese erst nach dem Durchzug der nordischen Vögel in den mitteleuropäischen Brutgebieten eintreffen. In der Phänologie der Ankunft von Wiederfängen zeigen nach oben abweichende Fangzahlen an $(=$ mehr Wiederfänge erhalten, als durch Erwartungswert berechnet), wann ihr Haupteinflug stattfindet.

M: Für jede Dekade wurden Erwartungswerte aus dem Anteil der Fänglinge in Relation zum Gesamtfang berechnet und mit den beobachteten Fangwerten verglichen, um Abweichungen festzustellen (Abb. 15).

E: In keiner Dekade konnte eine signifikante Abweichung der Wiederfänge beim Zilpzalp festgestellt werden, dazu hätte der beobachtete Wert außerhalb der oberen bzw. unteren Schranke liegen müssen (Abb. 15). 


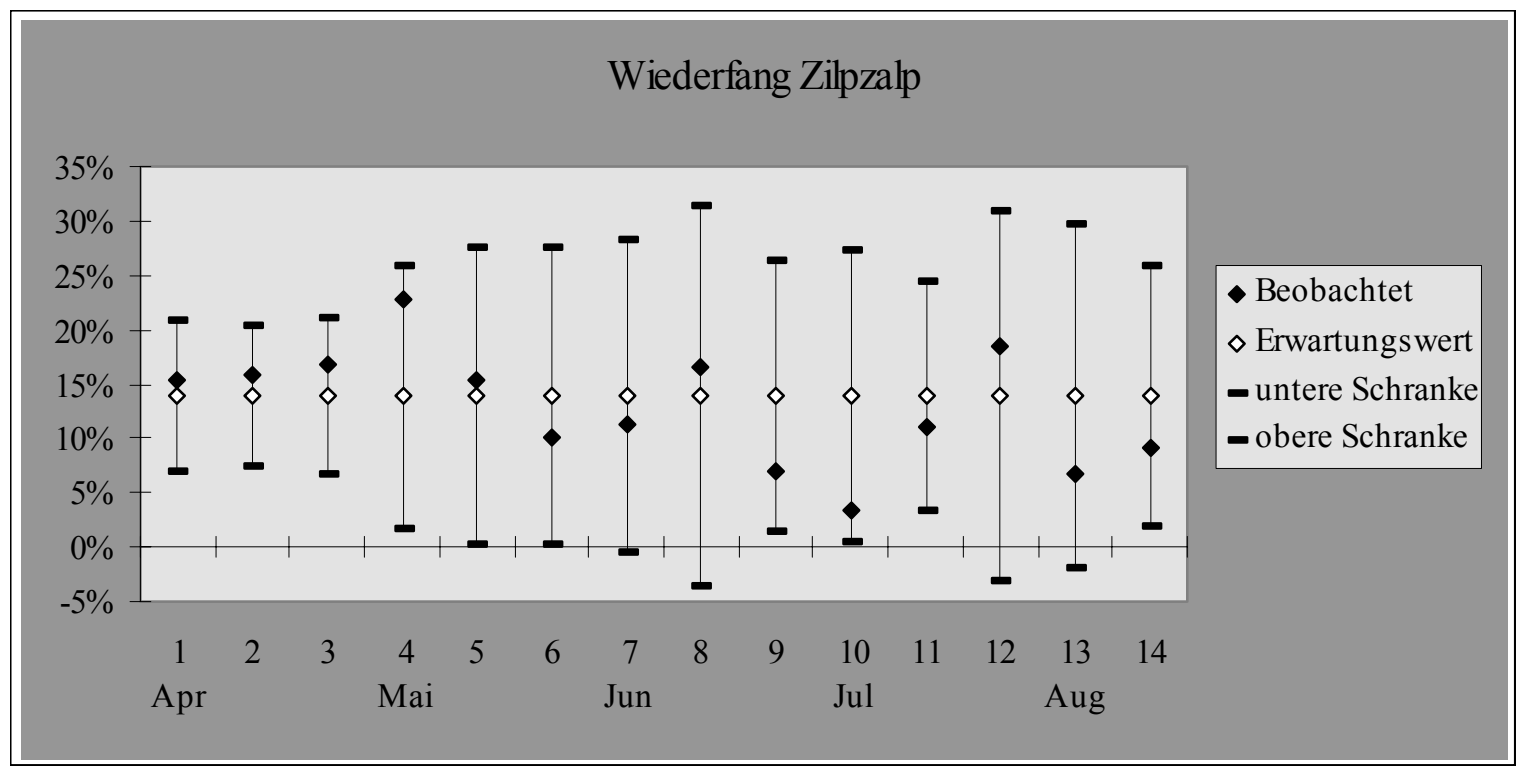

Abb. 15: Beobachteter Anteil der Wiederfänge des Zilpzalps im Dekadenvergleich mit dem Anteil adulter Erstfänge (Erwartungswert) über KFA. Die Werte sind zur besseren Vergleichbarkeit durch die Grundgesamtheit geteilt.

D: Der Einzug der Brutvögel erfolgte zeitgleich mit dem Hauptstrom durchziehender Zilpzalpe (Abb. 10). Der relative Anteil von Wiederfängen erreichte nach dem Hauptdurchzug in der ersten Maidekade ein Maximum. Die zu diesem Zeitpunkt beringten Vögel können jedoch schon eine unbestimmte Zeit vorher in den Hecken angekommen sein. Der erhöhte Anteil dürfte auf dem Abzug der durchziehenden Individuen beruhen.

Unter den im April auftretenden Zilpzalpen sind bereits $60 \%$ der späteren Revierinhaber (Abb. 14). Nur im April liegen alle beobachteten Werte über dem Erwartungswert (Abb. 15). Für den praktischen Gebrauch und die Interpretation von Kartierungsergebnissen lässt sich schließen, dass unter den im April erfassten Zilpzalpen bereits viele Revierinhaber sind, auch wenn noch starker Durchzug stattfindet.

Für eine genauere Abtrennung der Fangzahlen von späteren Revierinhabern ist die Skalierung von zehntägigen Intervallen zu grob. Die Ankunft der ersten Individuen wird aufgrund des Fangbeginns am 1. April nicht erfasst, denn Zilpzalpe können bereits ab März in den mitteleuropäischen Brutgebieten erscheinen (HELBIG \& DiERSCHKE 2000).

\subsubsection{Wieviel Zeit liegt zwischen dem Erstfang und dem Wiederfang?}

Bei der Durchführung von Monitoring-Programmen ist man aus verschiedensten Gründen bemüht, den zu leistenden Aufwand so gering wie möglich zu halten. Die Qualität der Ergebnisse darf dabei nicht vermindert werden. Im Integrierten Monitoring von Singvogelpopulationen (IMS, BAIRLEIN et al. 2000) ist die Fangperiode in der Brutzeit um zwei Dekaden von 14 auf 12 verkürzt. Unter diesem Gesichtspunkt stellt sich die Frage nach 
möglichen Auswirkungen der Einengung des Zeitfensters, in welchem ein standardisierter Netzfang durchgeführt wird.

M: Mit der Verringerung der Fangtage sinkt die Wahrscheinlichkeit, Wiederfänge zu erhalten. Bei einer Verkürzung von 14 auf 12 Fangtage wäre bei gleicher Fangwahrscheinlichkeit von 7,7\% in allen Dekaden (mit Ausnahme der ersten) mit einem Rückgang von 15,4\% der Wiederfänge zu rechnen. Im Folgenden wurde exemplarisch am Zilpzalp überprüft, wie die Erfassung von Wiederfängen über die Zeit nach der Erstberingung verteilt ist (Abb. 16).

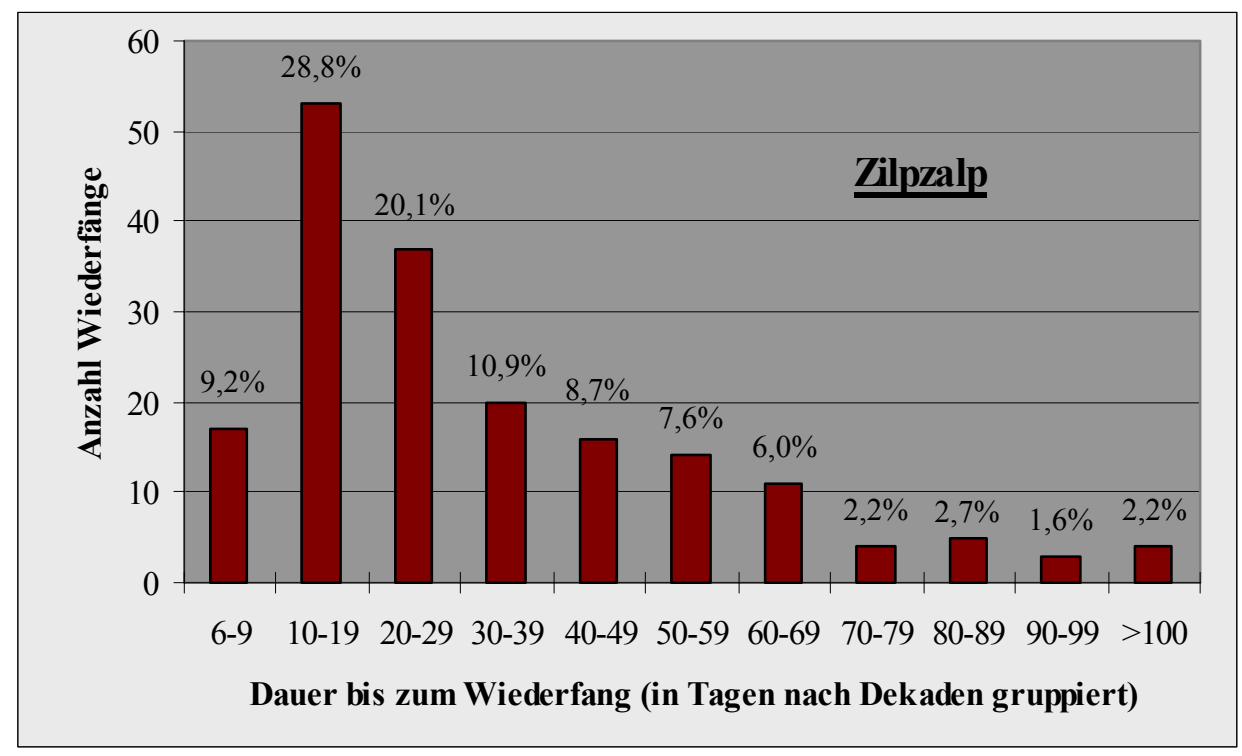

Abb. 16: Zeiträume zwischen Erstfang und Wiederfang beim Zilpzalp zwischen 1994-1998 in 23 Hecken $(n=184)$.

E: Die Verteilung über die Dekaden zeigt, dass die meisten Wiederfänge kurz nach dem Zeitpunkt der Beringung erfolgen. Innerhalb der ersten 29 Tage werden bereits annähernd $60 \%$ der Wiederfänge gemacht. In den folgenden 10 Tagen kommen weitere $10 \%$ hinzu. In den letzten Dekaden (70->100 Tage nach der Beringung) werden nur noch 8,7\%, statt zu erwartender $30,8 \%$, von allen Wiederfängen registriert.

D: Da die Wiederfangwahrscheinlichkeit kurz nach dem Beringungstag erheblich höher ist, als zu späteren Zeitpunkten, kann unter diesem Gesichtspunkt der Zeitraum der Fangperiode verkürzt werden. Eine Verkleinerung des Zeitfensters um zwei Dekaden, wie beim standardisierten Fang von Singvögeln im IMS festgelegt, lässt keine wesentlich geringere Fangzahl (maximal $2 \%$ Abnahme) von Wiederfängen beim Zilpzalp erwarten. Es ist zudem zu erwarten, dass die Verkürzung der Fangperiode zu einer besseren Motivation und Disziplin der Einhaltung der 'wenigen' Fangdekaden führt. 


\section{Vergleich der Fangergebnisse mit den Daten der Revierkartierungen}

Die Kombination von Netzfang und Revierkartierung auf den Untersuchungsflächen ermöglicht einen Vergleich der Ergebnisse beider Methoden zur Beschreibung von Brutvogelbeständen. Populationsverläufe anhand von Wiederfängen lassen sich aufgrund geringer Stichprobengröße nur für einige Arten abbilden und mit den Ergebnissen der Kartierung vergleichen (6.2). Im Folgenden wird die Frage behandelt, ob die Ergebnisse des Netzfangs die Häufigkeit der Arten widerspiegelt, die auch durch die Revierkartierung gefunden wird, welche Arten in höherer oder geringerer Anzahl gefangen werden und auf welche Ursachen das zurückgeführt werden kann.

\subsection{Korrelation der absoluten Anzahl von Arten zwischen Fang und Kartierung}

E: Die Häufigkeit von Brutpaaren und Erstfängen der 38 Programmvögel korreliert signifikant positiv ( $\mathrm{r}_{\mathrm{s}}=0,962, \mathrm{~N}=38, \mathrm{p}<0,002$, zweiseitig). In Abb. 17 sind die Abundanzen von Kartierung und Fang für die häufigsten 30 Programmarten aufgetragen.

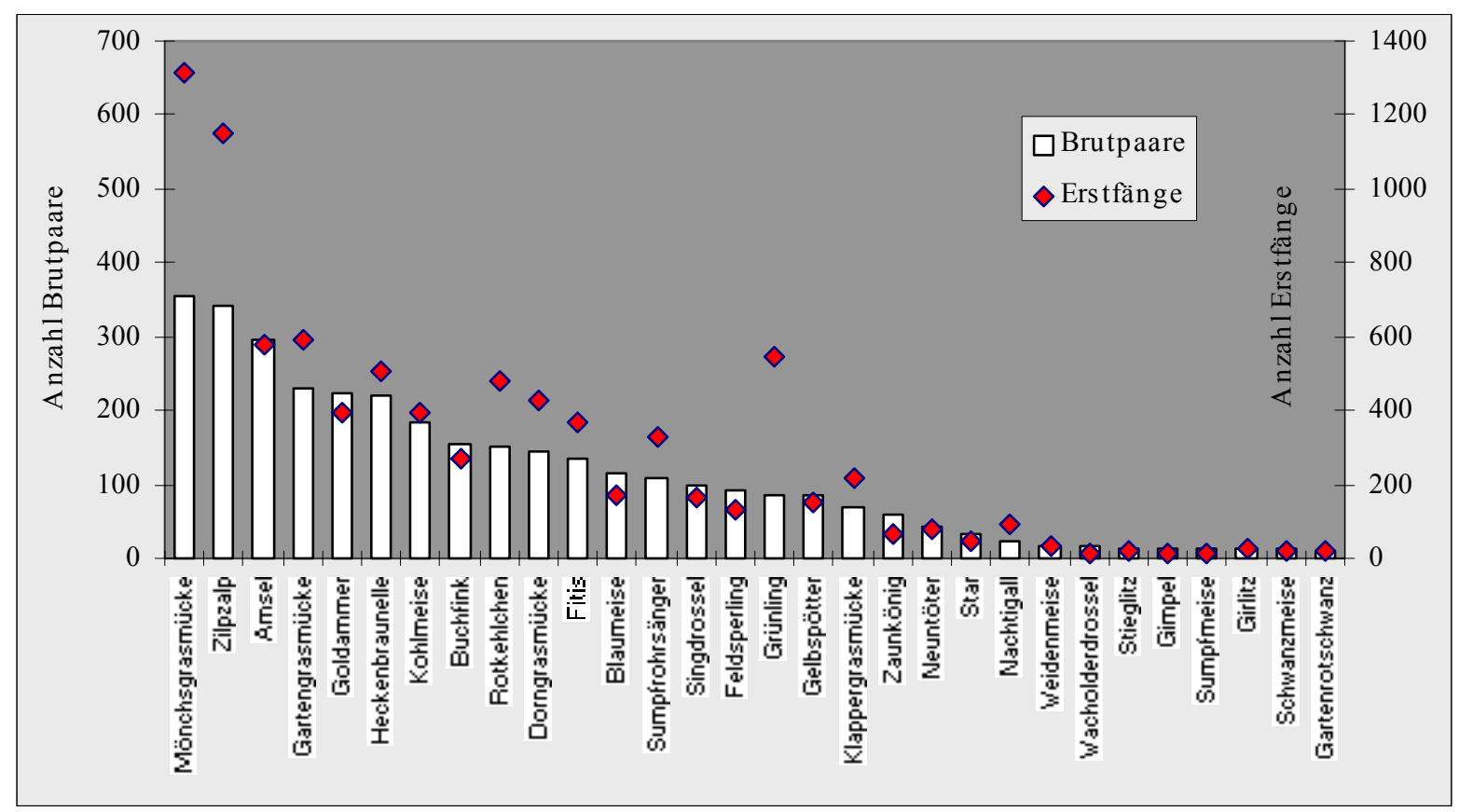

Abb. 17: Korrelation von Brutpaaren und Erstfängen der 30 häufigsten Programmvögel.

Die Skalierung der Ordinatenachsen ist so gewählt, dass jeweils ein Brutpaar zwei Erstfängen entspricht. Vor allem von ziehenden Arten wurden mehr Individuen durch Netzfang festgestellt, als das anhand der kartierten Brutpaare zu erwarten wäre, dazu zählen vor allem Mönchsgrasmücke und Zilpzalp, aber auch Gartengrasmücke, Heckenbraunelle, Rotkehlchen, 
Dorngrasmücke, Fitis, Sumpfrohrsänger, Klappergrasmücke und Nachtigall. Ausnahmen bildeten der Grünling und mit nur wenig höheren Fangzahlen als Brutpaaren, die Kohlmeise.

\subsubsection{Vergleich der Häufigkeitsverhältnisse der Arten zueinander (Dominanz)}

Die 18 häufigsten Programmvögel decken über $90 \%$ aller betrachteten Brutpaare $(91,2 \%)$ und Erstfänge (94,3 \%) ab und sind mit angegebener Dominanz in Tab. 19 dargestellt.

Tab. 19: Vergleich der Dominanz der 18 häufigsten Programmvögel.

\begin{tabular}{|l|r|r|}
\hline \multicolumn{1}{|c|}{ Art } & \multicolumn{2}{|c|}{ Dominanz } \\
\hline Mönchsgrasmücke & 10,48 & Erutpaare \\
Zilpzalp & 10,09 & $* 15,16$ \\
Amsel & $* 8,70$ & 613,26 \\
Gartengrasmücke & 6,82 & 6,67 \\
Goldammer & $* 6,55$ & 6,80 \\
Heckenbraunelle & 4,46 & 4,58 \\
Kohlmeise & 5,46 & 5,83 \\
Buchfink & $*, 60$ & 4,58 \\
Rotkehlchen & 4,49 & 3,11 \\
Dorngrasmücke & 4,28 & 5,50 \\
Fitis & 3,95 & 4,89 \\
Blaumeise & $* 3,42$ & 4,26 \\
Sumpfrohrsänger & 3,22 & 1,94 \\
Singdrossel & 2,89 & 3,77 \\
Feldsperling & $*, 69$ & 1,87 \\
Grünling & 2,57 & 1,52 \\
Geldbspötter & 2,48 & $* 6,30$ \\
Klappergrasmücke & 2,07 & 1,72 \\
\hline \multicolumn{1}{|c|}{ Summe [\%] } & 91,22 & 2,49 \\
\hline
\end{tabular}

* Z-Test zum Vergleich relativer Häufigkeiten signifikant nach Bonferoni-Korrektur

Fünf Arten werden signifikant häufiger als Brutvogel kartiert und drei Arten werden öfter in den Stellnetzen gefangen. Bei zehn Arten wurde kein Unterschied festgestellt.

D: Obwohl viele Zugvögel tendenziell höhere Fangzahlen als Brutpaare aufweisen (Abb. 17) haben von diesen nur Mönchsgrasmücke und Zilpzalp statistisch gesichert höhere Fangzahlen. Beide Arten sind Zugvögel und erscheinen auf dem Frühjahrszug in großer Anzahl in Hecken (vgl. Abb. 8 und Abb. 10). Die vielen Fänglinge des Grünlings erklären sich durch Einflüge von Trupps auf Nahrungssuche unter besonderen Bedingungen in der Umgebung von Hecken. Die Arten, die signifikant besser kartiert als gefangen wurden, sind relativ standorttreue Vögel. Blaumeisen siedeln sich bei einem Ortswechsel zwischen zwei Jahren innerhalb eines Radius von weit weniger als 100m wieder an (WINKEL \& FRANTZEN 1991). Die Ansiedlung der Jungvögel von Blaumeise und Feldsperling liegt in erheblich geringerer Entfernung zum Geburtsort an, als das bei ziehenden Arten (z.B. Trauerschnäpper) der Fall ist 
(WINKEL \& FRANZEN 1991, WinKEL 1993), so dass bei nicht ziehenden und standorttreuen Vögeln bereits die Erstbrüter über gute Ortskenntnis verfügen könnten. Es ist denkbar, dass ortstreue Individuen ihre Reviere besser kennen, als Individuen von Arten, die viel weniger Zeit in den Brutgebieten zubringen. Ortstreue Arten sollten Netze als neue Erscheinungen in den Revieren deshalb besser erkennen und meiden können.

Buchfink, Amsel und Goldammer zählen aufgrund ihres ausdauernden Gesangs, der zudem häufig von einer exponierten Warte aus vorgetragen wird, zu den am besten zu kartierenden Arten, weil sie in den Revieren sehr präsent sind und diese lange Zeit besetzt halten. Es ist anzunehmen, dass die Reviere dieser Arten sehr gut erfasst wurden. Durch den Netzfang sind diese Arten nicht so gut zu erfassen, weil der Buchfink sehr kleine Reviere bildet und dadurch von weit entfernt liegenden Netzstandorten nicht erfasst wird und weil Amsel und vor allem Goldammer außerhalb von Hecken Nahrung suchen und während dieser Zeit nicht im Fangbereichs der Stellnetze sind.

\subsection{Wie gut lässt sich der Brutvogelbestand anhand von Wiederfängen abschätzen?}

Um herauszufinden, ob anhand von Wiederfängen in der Brutzeit der Brutbestand in einem Fanggebiet abschätzbar ist, wurden die Kartierungsergebnisse die Anzahl von Wiederfänge für häufige Arten verglichen. Hecken bieten dazu gute Voraussetzungen, weil der Lebensraum sowohl für die Kartierung als auch als für den Netzfang eine gut abgrenzbare Untersuchungseinheit darstellt.

M: Zilpzalp, Mönchsgrasmücke, Heckenbraunelle und Amsel wurden ausgewählt, weil viele Wiederfänge vorlagen (Tab. 17). Goldammer und Dorngrasmücke wurden betrachtet, weil sie in Hecken mit einem Großteil ihrer mitteleuropäischen Populationen siedeln und eng an diesen Habitattyp gebunden sind. Obwohl auch Höhlenbrüter wie Kohl- oder Blaumeise eine hohe Anzahl an Wiederfängen aufwiesen, wurde auf eine Betrachtung dieser Arten verzichtet, weil nicht auszuschließen war, dass Fänglinge bei Nestkontrollen beringt oder abgelesen worden sind.

Die Gesamtzahl der Fangtage und deren Verteilung über die Dekaden kann einen Einfluss auf die Summe von Wiederfängen haben. Die durchschnittliche Anzahl von Fangtagen beträgt in den Jahren 1995, 1996 und 1998 jeweils 12,3 im Jahr und erreicht lediglich 1997 einen Wert von 12,6. Weil 1994 nur 10,8 Fangtage im Durchschnitt durchgeführt wurden, wurde auf die Darstellung des ersten Untersuchungsjahres verzichtet. Die geringe Abweichung in der 
Anzahl durchgeführter Fangtage 1997 wurde zu Gunsten einer Wiedergabe von realen Anzahlen der Wiederfänge und Brutpaare nicht durch Faktorisierung korrigiert.

Um eine direkte Vergleichbarkeit mit den Brutpaaren herzustellen, wurde bei Arten mit Geschlechtsdimorphismus die Anzahl der männlichen Wiederfänge berücksichtigt (Mönchsgrasmücke, Amsel und Goldammer) oder die Anzahl aller Wiederfänge halbiert.
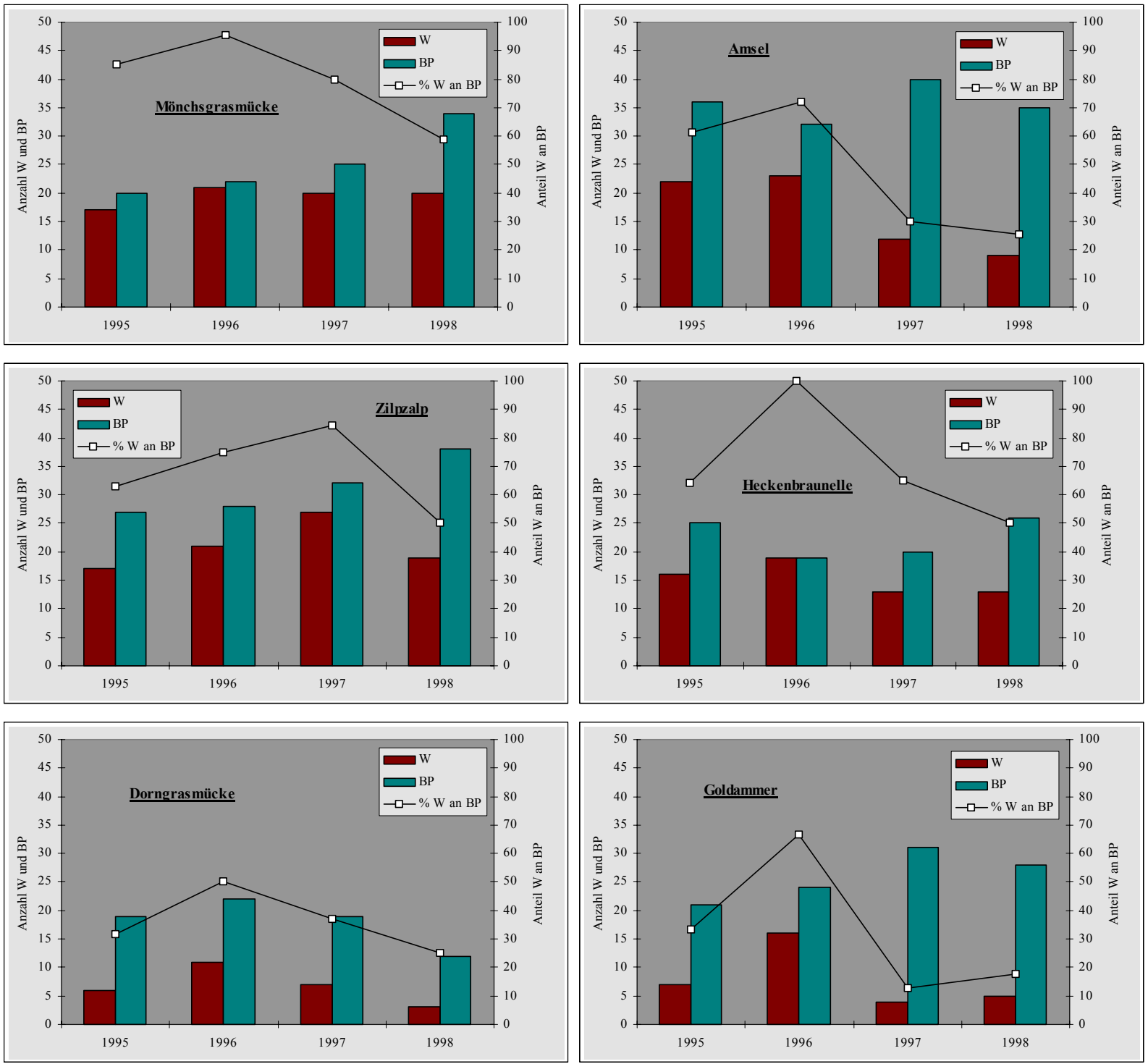

Abb. 18: Wiederfänge [W] und durch Revierkartierung ermittelte Brutpaare [BP] sowie der Anteil von Wiederfängen an den Brutpaaren [\% W an BP] ausgewählter Arten.

E: In Abb. 18 sind Wiederfänge und Brutpaare sowie der Anteil der Wiederfänge an den Brutpaaren dargestellt. Bei einigen Arten erreichen die Wiederfänge eine ähnliche Anzahl wie die der erhobenen Brutpaare (z.B. Mönchsgrasmücke), wenn auch oft nur in Einzeljahren (z.B. Heckenbraunelle 1996, Zilpzalp 1997). Für die genannten Arten liegt der Anteil der Wiederfänge an den Brutpaaren immer zwischen 50-100\%. In keinem Jahr wurden mehr Wiederfänge als Brutpaare registriert. Der Wert für den Anteil der Wiederfänge an den 
Brutpaaren ist jährlichen Schwankungen unterworfen. Mit Ausnahme der Goldammer wird 1998 der geringste Anteil von Wiederfängen im Verhältnis zu den Brutpaaren festgestellt.

Bei der Dorngrasmücke verliefen $\mathrm{Zu}$ - und Abnahmen über die vier Jahre parallel. Bei den anderen Arten sind immer Jahre, in denen Wiederfänge zunahmen und Brutpaare abnahmen oder umgekehrt (z.B. Goldammer und Amsel von 1996 auf 1997, Mönchsgrasmücke und Zilpzalp von 1997 auf 1998).

Die meisten Arten zeigen ein Maximum des prozentualen Anteils der Wiederfänge an den Brutpaaren (\% W an BP) im Jahr 1996. In diesem Jahr wurde im Fangprogramm der höchste Fangerfolg erzielt (vgl. Tab. 11). Hier zeigt sich eine mögliche Abhängigkeit der Anzahl von Wiederfängen vom allgemeinen Fangerfolg. Das gilt für Arten, die weniger häufig gefangen werden als Zilpzalp und Mönchsgrasmücke. Für letztgenannte Arten ist kein Zusammenhang zwischen dem Anteil der Wiederfänge und der Anzahl aller Fänglinge auf eine Summe von Fangtagen festzustellen. Beide Arten weisen 1998 die höchste Anzahl von Erstfängen (über 100 Fangtage berechnet, Tab. 11) auf, die Wiederfänge bleiben im Vergleich zum Vorjahr konstant oder nehmen sogar ab (Zilpzalp, Abb. 15). Dass für beide Arten in diesem Jahr der höchste Bestand an Brutpaaren gefunden wird, kann mit dem hohen Fangerfolg zusammen hängen: Durch die hohe Fangzahl wird die Anwesenheit vieler Individuen nachgewiesen. Da aber sowohl für Mönchsgrasmücke als auch für Zilpzalp kein Anstieg der Wiederfänge festzustellen ist, lässt sich vermuten, dass unter den Fänglingen ein großer Anteil von Durchzüglern ist. Diese sind möglicherweise von den Mitarbeitern als Revierinhaber registriert worden, was zu einer Überschätzung der Brutpaare geführt haben könnte. Hier liegt ein generelles Problem der Erfassung von Brutrevieren, denn es gibt keine Methode mit der ein Brutbestand 'genau' bestimmt werden kann, zumal dieser während der Brutsaison auch ständigen Veränderungen unterworfen ist (z.B. BERTHOLD 1976, BAIRLEIN 1978, BIBBY et al. 1992, GNIELKA 1992). Die Möglichkeit der Überschätzung von Populationen ist gerade dann groß, wenn die betreffenden Arten als Durchzügler über eine längere Zeitspanne in potentiellen Brutgebieten erscheinen, was bei Kurzstreckenziehern wie Zilpzalp und Mönchsgrasmücke der Fall ist. Gerade in klein parzellierten Untersuchungsgebieten wird vielfach das Fehlen einer Kontrollmöglichkeit für die gefundenen Kartierungsergebnisse oder Möglichkeit zur Angabe oder Einschätzung des Fehlers bemängelt (OELKE 1981). Hier bietet die Erfassung von Wiederfängen eine gute Möglichkeit, Ergebnisse von Revierkartierungen zu überprüfen, um Abweichungen zu diskutieren. 


\section{Zusammenfassung Heckenprogramm}

Das Heckenprogramm der deutschen Vogelwarten diente der Beschreibung und Beobachtung von Vogelpopulationen im Lebensraum Hecke über einen Zeitraum von fünf Jahren. Für die Bestandserfassung an Vögeln war es in Deutschland neu, dass mit ehrenamtlichen Mitarbeitern ein erweiterter Ansatz zur Erfassung von Brutvogelpopulationen und deren Bruterfolg gewählt wurde: Vögel wurden standardisiert in Stellnetzen gefangen und individuell markiert, die Brutvogelbestände wurden zusätzlich mit der Methode der Revierkartierung erfasst. Optional konnte von den Bearbeitern eine Nestersuche durchgeführt werden. Das zeitlich begrenzt angelegte Heckenprogramm war als Pilotstudie des nun in Deutschland etablierten 'Integrierten Monitorings an Singvogelpopulationen' angelegt.

Die Ergebnisse der Studie lassen sich folgendermaßen zusammenfassen:

1. Ein integriertes Bestandsmonitoring mit standardisiertem Netzfang und Revierkartierung ist mit ehrenamtlichen Mitarbeitern, unter der Betreuung durch die Vogelwarten, durchführbar. Auf die Einhaltung der methodischen Vorgaben sollte zur Verbesserung der Datenqualität strenger geachtet werden.

2. In den von 23 Bearbeitern untersuchten 33 Hecken konnten 95 Vogelarten zur Brutzeit in den Jahren 1994 bis 1998 nachgewiesen werden. Die Auswertung wurde zur besseren Vergleichbarkeit auf 38 heckentypische 'Programmarten' beschränkt.

3. Die relative Häufigkeit der Programmarten stimmte zwischen Netzfang und Kartierung überein. Die häufigsten Arten waren Mönchsgrasmücke und Zilpzalp. Auf diese Arten entfielen über $25 \%$ aller Fänglinge.

4. Hecken mit hoher Brutpaardichte waren nicht gleichzeitig auch Hecken mit großer Diversität. Damit widersprachen sich zwei ansonsten gebräuchliche Kriterien zur Beurteilung der Qualität von Habitaten in Hecken.

5. In Hecken, die älter als 20 Jahre waren, wurden durch Netzfang höhere Abundanzen festgestellt, als in jüngeren. Breite, Höhe und Gehölzdichte hatten keinen Einfluss auf die Gesamtabundanz.

6. Die Bevorzugung verschiedener Strukturausprägungen von Hecken durch Vogelarten wurde analysiert. Hecken mit über acht Meter Höhe wurden von Mönchsgrasmücke, Zilpzalp, Rotkehlchen, Gartengrasmücke, Amsel, Buchfink und Trauerschnäpper bevorzugt, während in Hecken mit geringerer Höhe Sumpfrohrsänger, Dorngrasmücke, Grünling, Gelbspötter, Star, Nachtigall und Girlitz signifikant höhere Dominanz hatten. In 
Hecken mit lückigem Gehölzbestand hatten Gartengrasmücke, Dorngrasmücke, Sumpfrohrsänger, Feldsperling, Gelbspötter und Neuntöter höhere Dominanz, Grünling, Rotkehlchen, Klappergrasmücke, Blaumeise und Schwanzmeise dagegen in geschlossenen. Mönchsgrasmücke, Star, Nachtigall, Gimpel und Trauerschnäpper bevorzugten breite Hecken (über $6 \mathrm{~m}$ ), Buchfink, Grünling und Neuntöter dagegen schmalere. In alten Hecken (über 20 Jahre) war die relative Häufigkeit von Mönchsgrasmücke, Zilpzalp, Gartengrasmücke, Rotkehlchen und Klappergrasmücke höher, in jüngeren Hecken waren Dorngrasmücke, Grünling, Zaunkönig, Star, Gimpel, Nachtigall, Girlitz und Bluthänfling häufiger.

Aufgrund dieser artspezifisch festgestellten und unterschiedlichen Präferenzen lässt sich kein Zieltypus einer 'Optimalhecke für Vögel' begründen.

7. Durch die Bevorzugung bestimmter Heckenstrukturen ließen sich die in den Hecken siedelnden Vögel gruppieren. Waldartige Strukturen in Hecken wurden von Rotkehlchen, Mönchsgrasmücke, Zilpzalp, Klappergrasmücke, Trauerschnäpper, Amsel, Blaumeise und Schwanzmeise, halboffene Strukturen dagegen von Dorngrasmücke, Sumpfrohrsänger, Neuntöter, Gelbspötter, Feldsperling, Girlitz, Bluthänfling und Zaunkönig bevorzugt.

8. Die Bestandsentwicklung häufiger Arten wurde dargestellt. 1996 hatten alle betrachteten Arten höhere Dichten als im Vorjahr, während 1997 für die meisten Arten weniger Altvögel festgestellt werden konnten. 1998 hatte die Mehrzahl wieder einen Bestandsanstieg. Bei den Jungvögeln war die Bestandsentwicklung über die Arten weniger signifikant. 1997 waren die meisten Zunahmen (12 Arten) und die wenigsten Abnahmen (5) festzustellen.

9. Die Fangphänologie von April bis Mitte August wurde als allgemeines Muster über alle Arten abgebildet. Die Fangzahlen von Jungvögeln waren mit weniger als 100 Individuen in 25 Hecken über fünf Jahre bis zum 20. Mai noch sehr gering und deuten auf sehr geringen Bruterfolg zu Beginn der Brutzeit hin. Abweichungen und Übereinstimmungen mit artspezifischen Mustern wurden diskutiert.

10. Anhand der Relation von Jungvögeln zu Altvögeln konnte der jährliche Bruterfolg indiziert und über die Jahre verglichen werden. Jahre mit hohem Bruterfolg (1995, 1997 und 1998) liegen nicht in den Jahren höchster Abundanz adulter Erstfänge (1996). Die Bedeutung der Bruterfolgskontrolle zur Bewertung von Habitaten wird anhand der Daten diskutiert. Faktoren die den Bruterfolg beeinträchtigen wurden genannt und erläutert. 
11. Von allen Programmarten wurden durchschnittlich $15 \%$ der Erstfänge nochmals in der Brutperiode gefangen. Die Heckenbraunelle hatte mit $28 \%$ die höchste Wiederfangrate unter den Arten mit mehr als 50 Wiederfängen insgesamt. Die höchste absolute Anzahl lag beim Zilpzalp mit 234 Wiederfängen vor. 1995 und 1996 war der prozentuale Anteil von Wiederfängen höher als 1997 und 1998. Die jährlichen Veränderungen des Anteils der Wiederfänge wurden mit Veränderungen der Erstfänge verglichen und diskutiert. Wiederfänge und kartierte Reviere zeigten bei einigen Arten Übereinstimmung. Bei hohen Anzahlen durchziehender Individuen im Frühjahr kann die Anzahl der Wiederfänge zur Vermeidung von Überschätzungen des Brutbestandes herangezogen werden.

12. Anhand von Wiederfängen des Zilpzalps konnte gezeigt werden, dass die Verteilung der Erstberingungen nicht von der allgemeinen Fangphänologie der Art abwich. Über 60 \% der später wiedergefangenen Individuen wurden bereits im April beringt. Zwischen der Beringung und dem Wiederfang vergingen für etwa $60 \%$ der Zilpzalpe weniger als 30 Tage.

13. Aus den gefundenen Ergebnissen aus Heckenbeschreibung, Brutbestandskartierung und standardisiertem Netzfang wurden Empfehlungen zur Pflege und Entwicklung von Hecken abgeleitet, wobei folgende Ergebnisse im Allgemeinen zu beachten wären:

Keine Hecke wird den Habitatansprüchen aller Arten gerecht werden.

Arten, für die eine Hecke Lebensraum bieten soll, müssen ausgewählt werden. Es bieten sich hierfür Arten an, die als Bewohner halboffen strukturierter Landschaften charakterisiert sind, z.B. Dorngrasmücke, Neuntöter, Bluthänfling und andere.

Die Entscheidung über die Habitateignung einer Hecke sollte über den Bruterfolg der Arten erfolgen und nicht primär oder ausschließlich über Artenzahlen oder Abundanzen.

Alte Hecken sind in ihrer Bedeutung für Vogelbestände nicht durch junge Hecken ersetzbar. 


\section{Populationsbiologische Bedeutung von Hecken für Vögel in der Kulturlandschaft}

\section{Einleitung}

Tierpopulationen werden in ihrer Dynamik und Entwicklung zunehmend von der Entfaltung menschlicher Aktivitäten beeinflusst. Dabei beeinträchtigt die immer intensiver werdende Nutzung der Landschaft das Überleben von Arten, weil intensive Nutzung zur Verschlechterung der Habitatqualität für viele Tierarten führt.

Hecken stellen naturnahe Lebensräume in intensiv genutzten Agrarlandschaften dar. In einer heckenreichen Kulturlandschaft finden sich arten- und individuenreiche Biozönosen (Tischler 1948c, ZwöLFer et al. 1984, STREeTER et al. 1985, MÜller, J. 1989), die einen hohen Naturschutzwert haben (BLAB 1993, MüHLENBERG \& SLOWIK 1997).

Viele in Mitteleuropa selten gewordene Vogelarten haben in heckengeprägten Landschaften wichtige Lebensraumrefugien gefunden, wie z.B. Neuntöter, Raubwürger und Rebhuhn (TUCKer \& HeAth 1994). Dazu kommen weitere Arten, die ursprünglich in lichten Wäldern oder Waldrandhabitaten vorkamen, wie zum Beispiel Ringeltaube, Amsel oder Mönchsgrasmücke (BEZZEL 1982, RINGLER et al. 1997).

In evolutiven Zeiträumen betrachtet sind Hecken junge und zudem vom Menschen geschaffene und zu unterhaltende Formationen (ElLENBERG 1996). Die Entstehung der Knicklandschaft Schleswig-Holsteins wird etwa auf die Mitte des 18. Jahrhunderts datiert (Weber 1967, zit. in PuChSteIN 1980). Für Tier- und Pflanzenarten stellen Hecken daher neue Lebensräume dar. Hecken unterliegen einem fortwährenden Einfluss durch Nutzung oder pflegerische Maßnahmen, um in ihrer Struktur erhalten zu bleiben. Die Landnutzung in der Umgebung von Feldhecken wird zunehmend intensiver und durch hohen Einsatz von Pestiziden und Dünger für freilebende Arten lebensfeindlicher. Die linienhafte Heckenstruktur zwingt die Vögel 'in einer Reihe' zu brüten, so dass für die Nester offen brütender Arten ein erhöhtes Prädationsrisiko besteht. Aus diesem Zusammenhang leitet sich die zentrale Frage dieser Arbeit ab, ob Hecken tatsächlich gute Habitate sind, in denen Singvögel eigenständig überlebensfähige Populationen bilden können.

Der Bruterfolg einer Population ist im Zusammenhang mit der Mortalität der wichtigste Faktor zur Bewertung der Qualität eines Bruthabitats. Nur wenn die Reproduktion die natürliche Sterblichkeit ausgleichen kann und gleichzeitig nicht mehr Individuen abwandern als zuwandern wird die Population stabil bleiben oder wachsen (BAIRLEIN 1996a). Habitate 
die 'Populationsüberschüsse' erzeugen bezeichnet man als Source-Habitate (PULliAM 1988). In Sink-Habitaten übersteigt die Mortalität die Netto-Reproduktionsleistung (ebd.).

Der Bruterfolg offen brütender Singvogelarten wird am stärksten durch prädationsbedingte Nestverluste beeinträchtigt (RICKLEFFS 1969, MARTIN 1992). In Hecken kann Prädation zu außergewöhnlich hohen Nestverlusten führen. Erstens kann die Linearität des Lebensraums dazu führen, dass die Räuber Nester leichter finden, weil es weniger potentielle Neststandorte als in flächigen Habitaten gibt und zweitens ist die Nestdichte in Hecken sehr hoch, so dass eine Suche für Prädatoren sehr lohnend sein kann.

Hecken tragen zur Artenvielfalt in der Kulturlandschaft bei (TISCHLER 1948b, ZwÖLFER et al. 1984, STREETER et al. 1985, BlAB 1993), werden aber in Abhängigkeit von ihrer Geschichte, Struktur, Management, geographischer Lage und ihrer Umgebung in unterschiedlicher Artenzahl und Abundanz besiedelt (Tischler 1948b, Blana 1978, PuChSTEIn 1980, ZWÖLFER et al. 1981, BEZZEL 1982, ERDELEN 1984, RINGLER et al. 1997). In zahlreichen Arbeiten hat man vor allem in England den Zusammenhang zwischen Heckenstrukturen und der Sommervogeldichte herausgearbeitet (ARNOLD 1983, OsBORNE 1984, LACK 1987 und 1988, Green et al. 1994, PARISH et al. 1994 und 1995, MACDONALD \& JOHNSON 1995): Vor allem das durch Höhe, Breite und Länge bestimmte Gehölzvolumen ist eine wichtige Einflussgröße für die Siedlungsdichte und Artenzahl von Vögeln in Hecken (HINSLEY \& BELLAMY 2000). Je größer das Gehölzvolumen einer Hecke, desto größer sind Artenzahl bzw. die Dichte der Brutpaare. GreEN et al. (1994) werfen aber die Frage auf, ob es für die Arten, die Hecken bevorzugt als Bruthabitat nutzen oder auf diesen Lebensraum in Ermangelung natürlicher Lebensräume angewiesen sind, von Vorteil ist, wenn Hecken in dieser Richtung gemanaged werden.

Um dieser Frage nachzugehen wurden in dieser Studie die Brutvogelbestände in 30 Hecken über drei Jahre erfasst. Die Hecken und ihre Umgebung wurden beschrieben und mit den Bestandszahlen der Brutvögel in Beziehung gesetzt. Dabei stand nicht nur die Frage im Vordergrund, welche Heckenstrukturen die höchsten Brutbestandsdichten in Hecken ermöglichen, sondern auch, welche Arten von diesen Strukturen profitieren.

Im Zusammenhang mit Pflege- und Ausgleichsmaßnahmen werden Hecken im praktizierten Naturschutz häufig als 'Ersatzlebensräume' angelegt. Insbesondere vor dem Hintergrund gravierender Veränderungen in der landwirtschaftlichen Nutzung und der daraus resultierenden negativen Bestandsentwicklung vieler in der Agrarlandschaft lebenden Vogelarten (TUCKER \& HEATH 1994), sollten aus den Resultaten Zielvorgaben für einen verbesserten und effektiveren Biotop- und Artenschutz für den Lebensraum Hecke abgeleitet werden. 
Wie haben sich die Brutvogelbestände über die Zeit verändert und welche Arten sind bereits aus den Hecken als Brutvogel verschwunden? Die Erfassung oder Berechnung von Artenzahl, Siedlungsdichte, Dominanz und Diversität dient der Beschreibung und dem Vergleich, vor allem zu den Vogelbeständen im letzten Jahrhundert. Insbesondere das Verschwinden und der Rückgang von Arten wird registriert, um bei der Formulierung von Naturschutzzielen nicht alleine von dem Status Quo einer längst degradierten Landschaft ausgehen zu müssen.

Sind Hecken für Vögel nur Ausweichhabitate, weil alle 'besseren' Reviere schon besetzt sind oder werden Hecken regelmäßig genutzt? Mit der Kartierung der Reviere über drei Jahre sollte die Konstanz der Anzahl der Brutpaare und die Kontinuität der in den Hecken siedelnden Arten untersucht werden. Dadurch lassen sich Hecken von geringer Attraktivität ausmachen, wenn Reviere oder Hecken nur manchmal besiedelt werden. Dauerhaft besiedelte Hecken lassen nicht ausschließlich auf hohe Attraktivität oder gute Habitateignung schließen, weil bei hohem Populationsdruck auch Habitate von minderer Qualität über längere Zeit besiedelt werden können.

Anhand der Anwesenheit einer Art ist noch keine Aussage über die Qualität eines Habitats möglich (VICKERY et al. 1992c, MÜHLENBERG 1993). Es ist denkbar, dass in Hecken das Bild eines konstant erscheinenden Populationsniveaus nur durch stetige Zuwanderung von Individuen aus anderen Habitaten erzeugt wird (KREBS 1971) und dass Hecken immer wieder neu besiedelt werden (IMS \& YACCOZ 1997, STACEY et al. 1997). Entscheidend für die Qualität von Habitaten ist daher der darin erzielte Reproduktionserfolg (BAIRLEIN \& SONNTAG 1994, BAIRLEIN 1996a). Um herauszufinden, ob Singvögel in Hecken überlebensfähige Populationen etablieren können, wurde für offen brütende Arten über drei Jahre der Bruterfolg kontrolliert, um berechnen zu können, ob der in Hecken erzeugte Nachwuchs ausreichend hoch ist, um die natürliche Mortalität ausgleichen zu können.

Gelegeverluste durch Prädation vermindern den Bruterfolg zum Teil erheblich, wobei sich Prädationsmuster in Abhängigkeit von Landschafts- oder Habitatstrukturen unterscheiden können (z.B. MAJOR \& Kendal 1996, MARTIN 1988, MARTIN \& RoPer 1988, Martin 1993a, 1993c, MATESSI \& BOGLIANi 1999). In Bezug auf Hecken stellt sich die Frage, ob es Unterschiede von Nestverlusten in Hecken gibt, die auf die Ausprägung der Hecke oder deren Einbettung in die Landschaft zurückzuführen sind. Anhand von Studien mit Kunstnestern oder an echten Nestern konnte festgestellt werden, dass es sogenannte 'edge-effects' gibt, die eine höhere Prädationsrate an Waldrändern als im Innenbereich von Wäldern bedingen (GAtes \& Gysel 1978, Møller 1989, Paton 1994, Andrén 1995, Fenske-Crawford \& Niemi 1997, Niemuth \& Boyce 1997, Saracco \& Collazo 1999), insbesondere wenn 
keine Sukzessionsübergänge an den Waldrändern vorhanden sind (SUAREZ et al. 1997). Hecken werden häufig aufgrund ihrer Struktur als doppelte Waldränder bezeichnet (STREETER et al. 1985) und lassen somit einen extrem hohen Räuberdruck auf Vogelnester erwarten. In dieser Arbeit wurde der Frage nachgegangen, welche Heckenstrukturen besonders hohem Prädationsdruck unterliegen, um unter diesem Aspekt Empfehlungen für die Anlage und Pflege von Hecken ableiten zu können. In den Hecken sollten zunächst die wichtigsten Nestparameter erfasst werden, die die Ausraubwahrscheinlichkeit bestimmen.

Im Untersuchungsgebiet ist die Nestdichte zwischen Mitte bis Ende Mai am höchsten (eig. Daten aus Nestfunden nach systematischer Suche). Weil der Räuberdruck dichteabhängig ist (MARTIN 1988, RICKLEFFS 1989, MARINI \& WEALE 1997), lässt sich vermuten, dass in dieser Phase der Brutzeit auch die Verluste an Kunstnestern am höchsten sind. Mit einer dreimaligen Wiederholung der Ausbringung von Kunstnestern in einer Brutsaison sollte untersucht werden, ob die zeitlichen Muster wechselnde Prädationsintensität an Hecken aufzeigen.

Um Prädationsmuster besser interpretieren zu können, ist es günstig, die Hauptprädatoren an den Nestern zu kennen (Fenske-Crawford \& NiEmi 1997, Brown et al. 1998). Von vielen Autoren wird daher zumindest eine Liste potentieller Nesträuber zusammengestellt oder der Versuch unternommen, anhand von Nestzustand oder Schalenresten, Trittsiegeln oder Bissspuren in sogenannten Clay-eggs (künstliche Eier aus verformbarer Knetmasse) auf den Räuber zu schließen (z.B. SANTOS \& TELlERÍA 1992, VICKERY et al. 1992a, MARINI et al. 1995, Huhta et al. 1996, Keyser et al. 1998, SARgENT et al. 1998). Da aber die Mehrzahl der Spuren an zerstörten Nestern auf eine Vielzahl von Prädatoren zurückgeführt werden kann (MARINI \& MELO 1998), birgt dieses Vorgehen viele Fehlerquellen. Im Rahmen dieser Studie wurden zur Ermittlung der Nesträuber Fotoapparate eingesetzt, die im Moment der Eientnahme auslösten und somit eine eindeutige Bestimmung des Nesträubers ermöglichten.

In Kenntnis der Hauptprädatoren und der Bedeutung von Neststandortparametern wurde das relative Prädationsrisiko in Hecken auf Landschaftsebene untersucht. Dazu wurden Landschafts- und Heckenmerkmale erfasst und mit dem Anteil ausgeraubter Nester in den Hecken in Beziehung gesetzt. Diese Muster dienten letztendlich auch der Interpretation der Ergebnisse an den 'echten' Nestern, die in dieser Studie kontrolliert wurden. 


\section{Untersuchungsgebiet}

Die Untersuchungen wurden in der Kulturlandschaft um Göttingen ( $\left.51^{\circ} 33^{\prime} \mathrm{N}, 09^{\circ} 57^{\prime} \mathrm{E}\right)$ in Südniedersachsen durchgeführt. Das Landschaftsbild ist geprägt von landwirtschaftlichen Nutzflächen und eingestreuten Wäldern, die vor allem um kleinere Bergrücken (Erhebungen um 250-400 m mit Buntsandstein oder Oberem Muschelkalk) etwa bis 100jährige Bestände bilden. Die Waldflächen werden intensiv forstwirtschaftlich genutzt, ohne dass Kahlschlagwirtschaft betrieben wird. Die Region wird vom Leinetalgraben durchzogen, der sehr fruchtbare Böden bietet. Das Verhältnis von 54,4 \% landwirtschaftlicher Nutzfläche zu einem Waldanteil von 33,3 \% (Landkreis Göttingen 1998) im Untersuchungsgebiet entspricht in etwa dem Bundesdurchschnitt dieser Bodennutzungstypen (55\% zu $29 \%$ ). Somit kann das Untersuchungsgebiet als typisch für viele landwirtschaftlich geprägte Landschaftsteile Deutschlands gelten, die nicht alle Feldgehölze, Einzelbäume, Hecken und Waldstücke im Zuge von Flurbereinigungen verloren haben.

Die Auswahl der untersuchten Hecken ergab sich nach Gesichtspunkten der Erreichbarkeit (maximal $25 \mathrm{~km}$ von Göttingen entfernt), Zugänglichkeit (Erlaubnis der Feldwegenutzung durch Landkreis oder Stadt Göttingen sowie der verschiedenen Feldinteressentenschaften), Durchführbarkeit (Erlaubnis durch Besitzer, Land- und Jagdpächter) und Verfügbarkeit mehrerer geeigneter Hecken in jeweils einem von insgesamt sechs verschiedenen Untersuchungsgebieten.

Alle bearbeiteten Hecken sind typische 'Feldhecken', sie liegen innerhalb der landwirtschaftlich genutzten Flächen. Daran entlang führende Straßen und Wege sind vorwiegend als nicht öffentliche Feldwege, zum Erreichen von landwirtschaftlichen Nutzflächen, angelegt worden. Gartenhecken, Alleen und 'Straßenbegleitgrün' wurden nicht untersucht. Die Ausprägung der untersuchten Hecken reichte von sehr lückigen Heckenfragmenten über junge, zum Teil sehr dichte Anpflanzungen bis zu reich strukturierten Doppelhecken.

Als Hecke sind linear angelegte Gehölzstrukturen definiert, die Strauchpflanzen enthalten. Bäume und Säume aus Wildkrautpflanzen können vorhanden sein.

Zur Abgrenzung von flächigen Anpflanzungen oder punktuellen Strukturen sei linear definiert als Strukturform deren Länge die Breite um mindestens das Dreifache übertrifft.

\subsection{Charakterisierung der untersuchten Heckenlandschaften}

Insgesamt wurden 30 Hecken in fünf verschiedenen Gebieten untersucht. Eine detaillierte Darstellung aller untersuchten Hecken sprengt den Rahmen dieser Arbeit. Es soll lediglich 
eine Beschreibung der großräumigen Untersuchungsflächen erfolgen, auf denen jeweils mehrere Hecken bearbeitet worden sind. Hecken die zusätzlich für die Nestersuche aufgesucht wurden oder in die Prädationsexperimente einbezogen wurden, entsprachen der allgemeinen Charakterisierung.

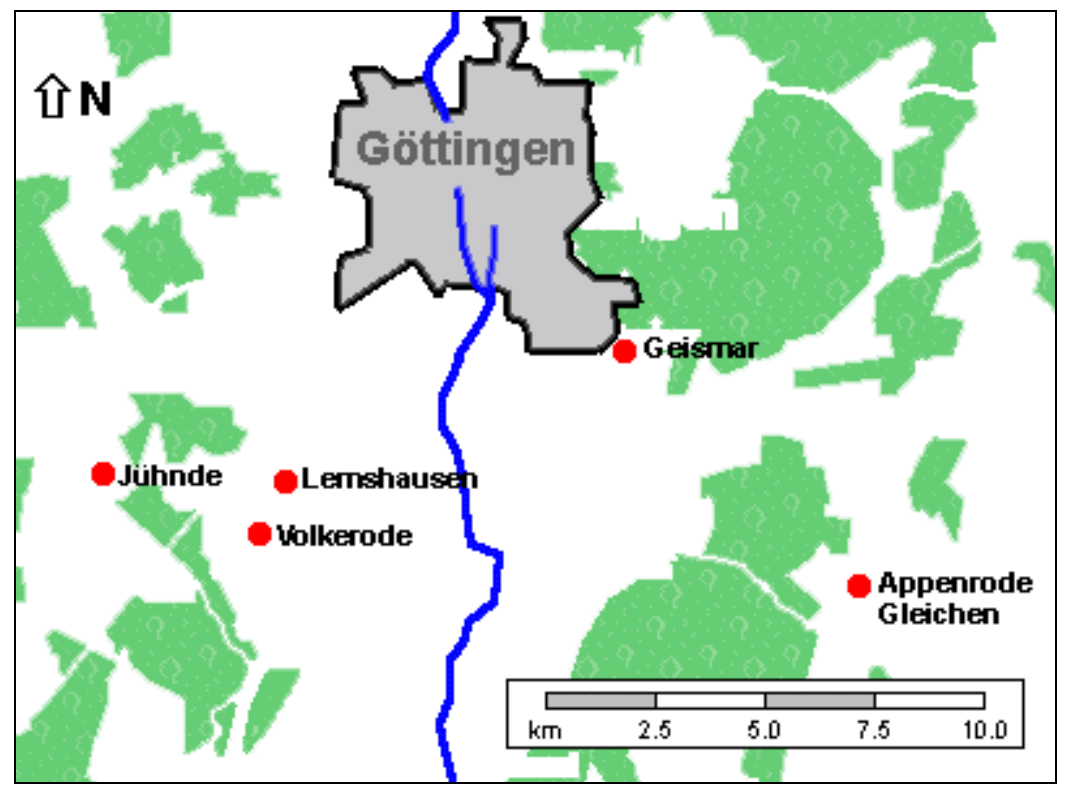

Abb. 19: Übersichtskarte der Untersuchungsgebiete um Göttingen.

Geismar (4 bearbeitete Hecken): Diese Hecken grenzen südöstlichen an Göttingen. Der Anteil von Bracheflächen liegt bei etwa $25 \%$, während auf den intensiv bewirtschafteten Ackerflächen vorwiegend Wintergetreide und Raps angebaut werden. Die häufigste Strauchart ist die Schlehe, in einigen Hecken bildet alter Weißdorn baumartige Überhälter. Eine Hecke ist von Pappeln durchwachsen und enthält einen Bestand von locker stehenden Apfelbäumen. Aufgrund der Stadtnähe werden die Feldwege an den Hecken sehr häufig durch Spaziergänger und Sportler genutzt.

Volkerode (15): Die um den südwestlich von Göttingen gelegenen Ort befindlichen Hecken sind bis auf eine Ausnahme über 50 Jahre alt. Die Zusammensetzung blieb ohne die Entfernung von Gehölzstrukturen wohl seit über 100 Jahren erhalten, weil keine umfassenden Flurbereinigungen in dieser Region durchgeführt worden sind, wie aus alten Aufzeichnungen und Karten der lokalen Feldmarksinteressentenschaft hervorgeht. Mit der Rodung vieler Streuobstbestände fand jedoch eine auffällige und starke Veränderung in der landwirtschaftlichen Nutzung, hin zu intensiverem Getreideanbau, statt.

Die Hecken sind von Schwarzdorn (Prunus spinosa) dominiert, zum Teil über $10 \mathrm{~m}$ breit und in einigen Abschnitten im Innenbereich unbelaubt und reich an abgestorbenem Geäst. Teilweise sind Kiefern (Pinus silvestris) in den Hecken aufgewachsen und verleihen den 
betreffenden Stellen Feldgehölzcharakter. Weißdorn (Crataegus spec.) und Hundsrose (Rosa cannina) sind weitere häufige Arten. Der Krautsaum ist dominiert von Süßgräsern und stellenweise dichten Beständen der Brennessel (Urtica dioica). An lichteren Gehölzlücken treten vor allem Brombeeren (Rubus spec.) auf.

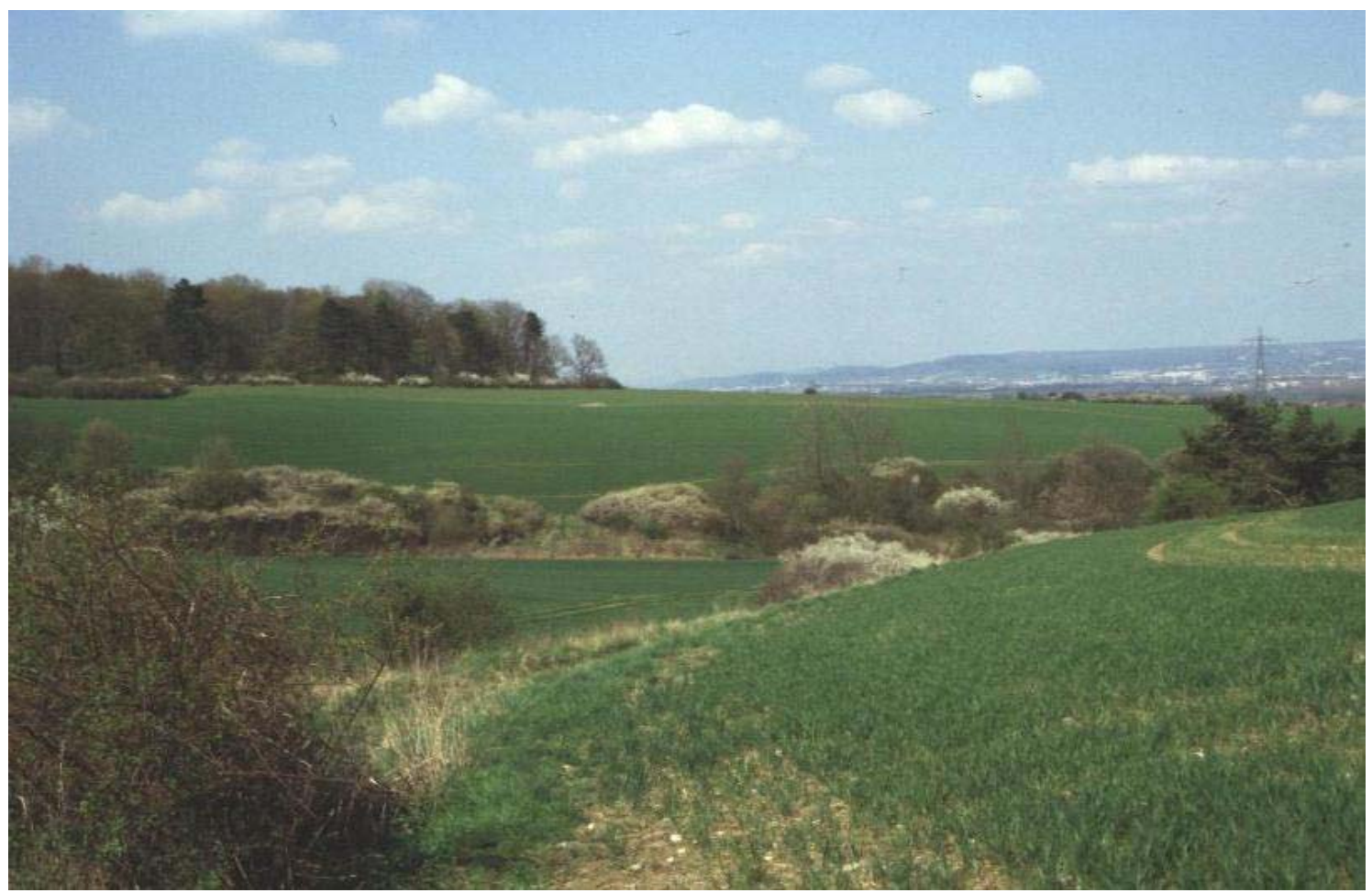

Abb. 20: Heckenlandschaft bei Volkerode am 21. April 2000: von Schlehen dominierte Hecken sind in intensiv genutzte Kulturlandschaft eingefügt, im Hintergrund liegt Göttingen.

Die landwirtschaftliche Nutzung ist konventionell ausgerichtet. Es werden vor allem Wintergetreide (Weizen, Roggen und Gerste) und Raps angebaut. Der Flächenanteil von Dauerbrachen ist mit etwa $20 \%$ relativ hoch.

Lemshausen (2): Entlang beider Hecken sind Reste ehemalig auch bei Volkerode reichlich vorhandener Streuobstflächen erhalten, die aufgrund kleinräumig wechselnder Besitz- und Bewirtschaftungsverhältnisse mosaikartig unterschiedliche Nutzung erfahren (z.B. kleinflächige Wiesenmahd, teilweise Beweidung mit wenigen Rindern, 25jährige Sukzessionsflächen auf einzelnen Parzellen). Es sind wegsäumende Doppelhecken ausgebildet, eine weitere Gehölzlinie verläuft entlang eines zeitweise wasserführenden Grabens. Hier bestimmen durchgewachsene Pappel (Populus spec.), Stieleiche (Quercus robur) und Schwarzer Holunder (Sambucus nigra) die Gehölzstruktur, entlang der Wege dominieren Prunus-Arten. Die Hecken sind von intensiv genutzten Landwirtschaftsflächen umgeben (siehe Heckenstruktur quer verlaufend im oberen Abschnitt in Abb. 21). 


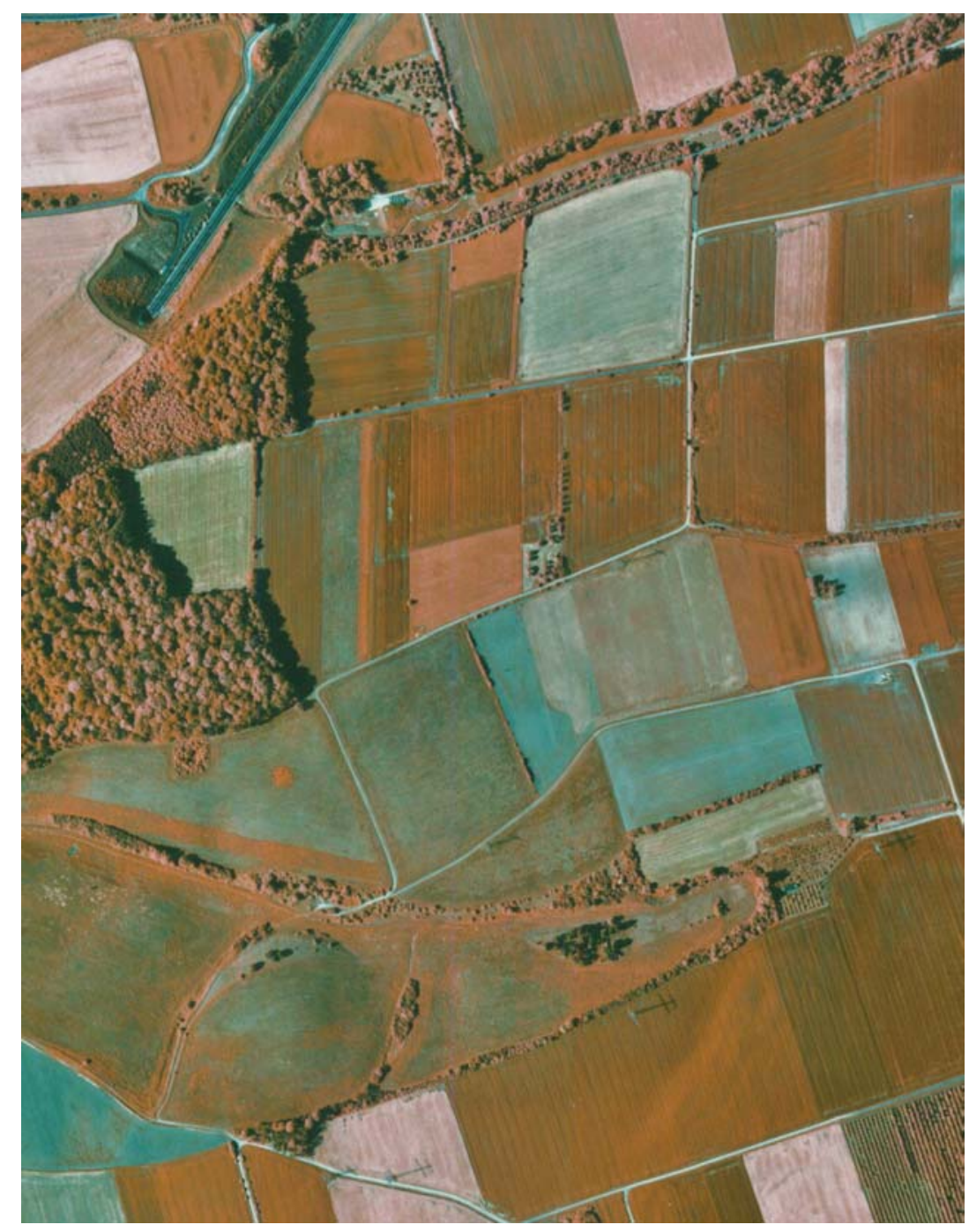

Abb. 21: Luftbild Volkerode im Maßstab 1:10.000 vom 17. Mai 1993

Appenrode-Gleichen (2): Die hier untersuchten Hecken liegen zwischen den Ortschaften Appenrode und Gelliehausen südöstlich von Göttingen. Die landwirtschaftliche Nutzung erfolgt ähnlich intensiv wie um Volkerode. Kleinere Flächen von Weiden und Mähwiesen sind vorhanden. Der Anteil von Bracheflächen liegt bei etwa $10 \%$. In den Hecken sind Weißdorn und Hundsrose häufiger als um Volkerode, wodurch die Struktur einiger Hecken hier 'aufgelockerter' erscheint.

Jühnde (7): Hier dominieren große Schläge mit Wintergetreide oder Winterraps. Eine ausgedehnte Brachefläche und zwei Mähwiesen grenzen an einige der Hecken. 


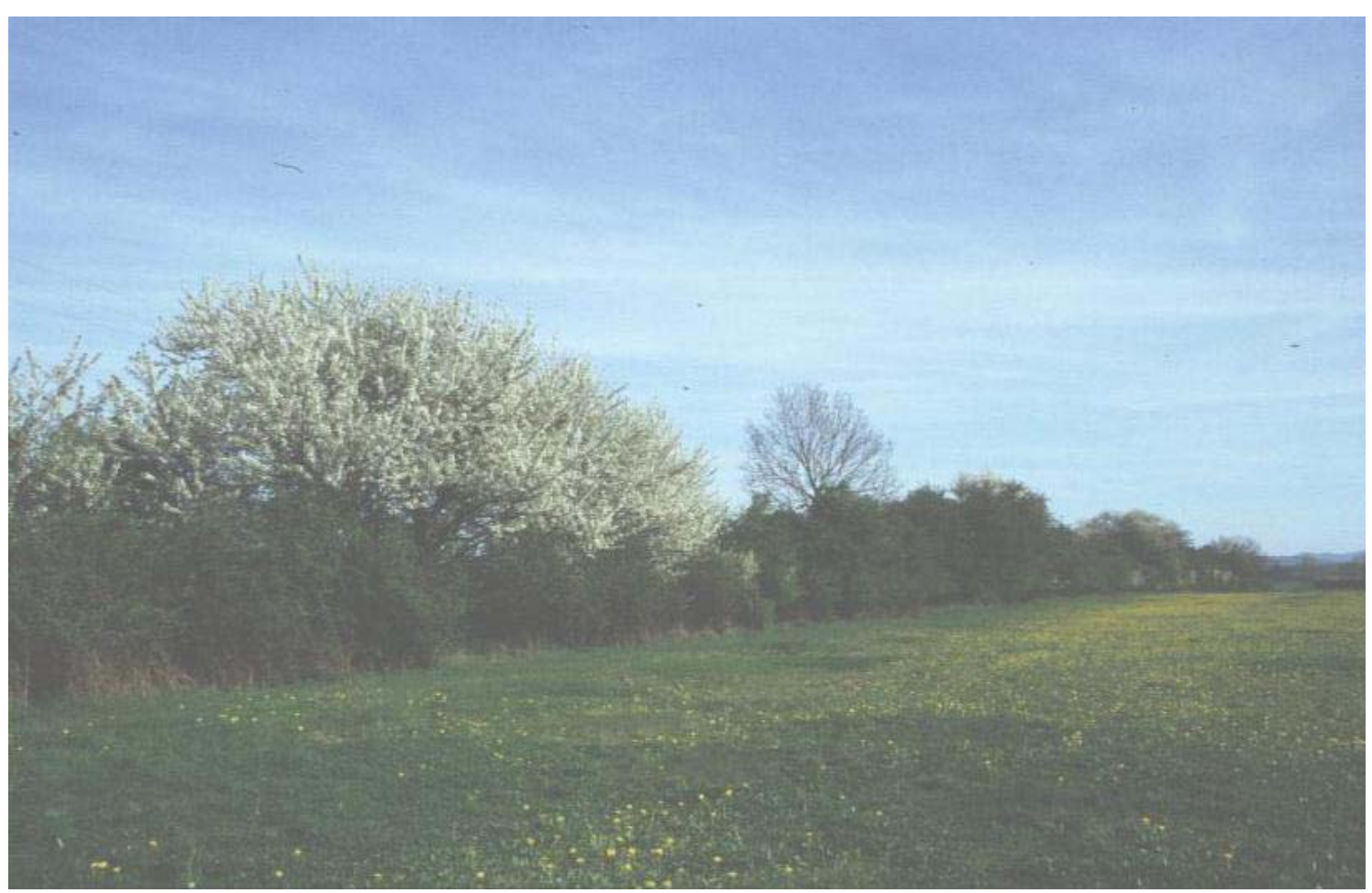

Abb. 22: Hecke 45 bei Jühnde, mit einer mehrjährigen Brachefläche im Vordergrund

Die hier bearbeiteten Hecken sind strukturell sehr unterschiedlich. Einige sind zumindest fragmentarisch als Doppelhecken ausgebildet, weitere als ehemals beidseitig angelegte Wegbegleitstrukturen mittlerweile zusammengewachsen und von vereinzelten Überhältern durchwachsen. Die Gehölzartendiversität ist in diesen Hecken vergleichsweise hoch, hier finden sich neben bereits erwähnten Arten das Europäische Pfaffenhütchen (Euonymus europaea) und die Gemeine Vogelkirsche (Prunus avium). Zwei waldartig angelegte Gehölzstreifen sind von Rotbuchen (Fagus sylvatica) dominiert, enthalten aber auch Kirsche, sowie Lärche (Larix decidua), Weißdorn, Eiche und Bergahorn (Acer pseudo-platanus) und sind an den Rändern von Schlehe und Hainbuche unterwachsen. 


\section{Methoden}

\subsection{Strukturkartierung an Hecken}

Zur Beschreibung der Hecken und ihrer Umgebung wurden Strukturparameter aus der vorhandenen Literatur zur Analyse von Beziehungen zwischen Vogelbeständen und Habitatstrukturen (z.B. GAßMANN \& GLÜCK 1993, GREEN et al. 1994, MACDONALD \& JOHNSON 1995) zusammengestellt und nach folgenden drei Kriterien für diese Studie ausgewählt:

1. Relevanz für Vögel zur Brutzeit (nach Literaturangaben und eigener Erfahrung)

2. Beschränkung auf wenige, aber charakteristische Merkmale (Nachvollziehbarkeit)

3. Einfachheit der Aufnahme im Gelände oder aus Kartenmaterial (Wiederholbarkeit)

Folgende Variablen wurden aufgenommen:

\section{$\underline{\text { Heckenstrukturen }}$}

Länge [m; Begrenzung durch schneidende Wege, landwirtschaftliche Nutzflächen oder neu zu beschreibende Heckenstruktur vorgegeben]

Breite [m; Messung der von Gehölzen auf Brusthöhe erreichten Breite, in jeweils 25 Meter Abstand vermessen und Bildung eines auf 0,5 Meter gerundeten Mittelwertes]

Krautsaum [m; Ausdehnung der krautigen Vegetation zwischen Gehölzrand und Weg oder Feldrand zu beiden Seiten und dann gemittelt, Messpunkte und Rundung wie bei Breite]

Überhälter [Anzahl überragender Bäume oder Sträucher auf Längeneinheit bezogen]

Heckendichte [\%; Gesamtlänge der Hecke abzüglich der Gehölzlücken]

$\underline{\text { Landschaftsparameter }}$

Siedlungsnähe [m; auf $10 \mathrm{~m}$ exakt]

Waldnähe [m; auf $10 \mathrm{~m}$ exakt]

Wegedichte [m; Weglänge auf 10 ha Landschaftsausschnitt]

Heckenabundanz [m; Hecke auf 10 ha Landschaftsausschnitt, Bezugshecke ausgeschlossen]

Heckenverbund [m; kürzeste Entfernung zur nächsten Heckenstruktur]

Acker [\%; an Hecke angrenzende Flächennutzung]

Brache [\%, an Hecke angrenzende Flächennutzung]

Die Heckenstrukturen wurden vor Ort erfasst, die Angaben zu den umgebenden Landschaftsstrukturen wurden auf Luftbild-Laserkopien im Maßstab 1:2.500 mit einem Planimeter (Digital Flächen/Längen-Planimeter RIEFLER Super PLANIX $\alpha$ ) erhoben. 


\subsection{Brutvogelbestandserfassung: Revierkartierung}

Die Kartierung der Vogelbestände und die Festlegung der gefundenen Reviere in den Hecken erfolgte nach den allgemeinen Richtlinien zur Durchführung von Revierkartierungen (BIBBY et al. 1995). Kartiert wurden alle optischen und akustischen Beobachtungen, insbesondere territoriale Verhaltensweisen und Hinweise auf Neststandorte oder vorhandene Jungvögel. Die schmaleren und dichten Hecken wurden langsam abgelaufen, breite Hecken darüber hinaus von innen durchlaufen. Die Routen wurden immer gewechselt. Kartierungsbeginn war in der Regel eine Stunde vor Sonnenaufgang, zusätzlich wurde an jeder Hecke mindestens eine Abendkartierung durchgeführt. Neben einer ausreichenden Anzahl von Kontakten (s. dazu MARCHANT 1983 in BIBBY et al. 1995) wurde auch der Fund eines belegten Nestes zur Feststellung eines Revieres genutzt. Die jeweils 8-14 Begehungen je Hecke und Jahr fanden zwischen März und Juli 1998 bis 2000 statt. Angaben zur Besiedlungsdichte sind aufgrund der linearen Strukturen als Revieranzahl je 1 km Hecke angegeben.

\subsubsection{Kumulative Artzusammensetzung in Hecken}

Vor dem Hintergrund der Bewertung von Habitaten nach der Anzahl vorkommender Arten in der gängigen Naturschutzpraxis erscheint es sinnvoll, der Frage nachzugehen, ob Hecken artenreich sein müssen, um einen hohen Naturschutzwert zu haben. Dazu wurde das Zustandekommen hoher Artenzahlen über das sukzessive Auftreten weiterer Arten beschrieben. Die Arten aller Hecken mit weniger als 10 festgestellten Arten wurden in einer Gruppe zusammengefasst. In die zweite Gruppe kamen die Arten, die erst in Hecken auftraten, die mehr als 10 aber weniger als 15 Arten aufwiesen. Darauf folgte eine Gruppe mit 15 bis 20 Arten und schließlich Arten die erst mit 20 und mehr Arten festgestellt wurden.

Dieses methodische Vorgehen impliziert keine Kausalität zwischen dem Auftreten von Arten in Abhängigkeit anderer Vogelarten, sondern soll die Frage beantworten, um welche zusätzlichen Arten es sich handelt, wenn man von artenreichen Hecken gegenüber anderen spricht.

\subsection{Statistik Heckenstrukturen und Brutvogelbestände}

Zur Ermittlung des Einflusses von Strukturvariablen auf Brutvogelbestände in Hecken wurde eine Multiple Lineare Regression durchgeführt. Die Rohdaten mussten dafür näherungsweise in eine Normalverteilung überführt werden. Transformiert wurden die Variablen Breite, Überhälter, Waldentfernung, Heckenverbund (Quadratwurzel) und Heckendichte (ArcSinTransformation). Die Variablen Acker und Brache konnten nicht in eine Normalverteilung überführt werden und mussten von der Analyse ausgeschlossen werden. Alle Daten wurden 
vor der Analyse normiert, um einen direkten Vergleich der Beta-Werte zu ermöglichen, damit die relative Stärke des Einflusses jeder ermittelten Variable abgelesen werden kann.

Mit einer Hauptkomponentenanalyse (üblicherweise PCA abgekürzt, von principal components analysis) wurde nach Variablenkomplexen gesucht, die stark miteinander korreliert sind und erst in ihrem Zusammenwirken nachweisbare Effekte auf Vogelbestände zeigen.

Eine kanonische Korrelation diente zur Bestimmung des Zusammenhangs von zwei Variablenkomplexen: der Heckenstruktur einerseits und der Abundanz einzelner Brutvogelarten andererseits.

Zur Analyse der Beziehung zwischen zwei Variablen (z.B. Artenzahl und Länge von Hecken) wurde der Spearman Rang-Korrelationskoeffizient $r_{s}$ berechnet.

Die Diversität wurde nach Shannon-Wiener (vgl. dazu ScHAEFER 1992, MÜHLENBERG 1993) gemäß der Gleichung

$$
H_{S}=-\sum_{i=1}^{S} p_{i} \log p_{i}
$$

wobei

$\mathrm{p}_{\mathrm{i}}=$ relativer Anteil der Art i an der Geamtindividuenzahl und

$\mathrm{S}=$ Gesamtzahl der Arten sei,

berechnet. Der Wert von $\mathrm{H}_{\mathrm{S}}$ als Diversitätsmaß wird mit zunehmender Artenzahl und bei Zunahme der Gleichverteilung der Abundanz der Arten größer.

Für alle statistischen Analysen wurde die Software STATISTIKA ${ }^{\circledR} 5.0$ von 1997 benutzt. Die Verfahren sind, soweit nicht anders vermerkt, entsprechend den Vorgaben in BORTZ (1995) und des Elektronischen Handbuchs in STATISTIKA ${ }^{\circledR}$ durchgeführt.

\subsection{Bruterfolg: Nestersuche}

Zur Ermittlung des Bruterfolgs wurden in 35 Hecken die Nester von frei brütenden Arten gesucht. Die Lage der Neststandorte wurde kartiert und zu jedem Nest, das in aktueller Nutzung war, wurde eine Nestkarte der deutschen Vogelwarten angelegt. Darauf wurden neben der Protokollierung der brutbiologischen Daten auch Angaben zu Lebensraum und Neststandort, zur Nest- und Vegetationshöhe festgehalten. Bei der Nestersuche und Datenaufnahme wurden die 'Richtlinien zur Erfassung brutbiologischer Daten mit Hilfe von Nestkarten' (Vogelwarten Radolfzell und Helgoland, ohne Jahr) und die Anleitung zum Ausfüllen von Nestkarten (ebd.) befolgt.

Die Erfassung brutbiologischer Daten dient, neben der Beschreibung der Brutphänologie, der Feststellung des Brutbeginns und der Anzahl der Jahresbruten, vor allem der Ermittlung des 
Bruterfolgs. Dazu ist es wichtig, den Legebeginn, die Anzahl der Eier des Vollgeleges, den Schlupftag und den Tag des Ausfliegens der Jungvögel und die jeweilige Anzahl der Eier bzw. Jungvögel zu wissen. Eine tägliche Kontrolle der gefundenen Nester war aufgrund der Vielzahl von untersuchten Hecken nicht möglich. In der Regel wurden die Nester alle drei bis sechs Tage kontrolliert.

Der Tag der Ablage des ersten Eies konnte erfasst werden, wenn ein Nest in der Legephase (Zeitraum in dem am Folgetag ein weiteres Ei abgelegt wird) gefunden oder kontrolliert wurde, unter der Annahme, dass die bearbeiteten Singvogelarten jeden Tag ein Ei legen. Legeunterbrechungen wurden nur in einem Fall (an einer Heckenbraunelle 1998) beobachtet und im Übrigen zur Feststellung des Legebeginns vernachlässigt. Das Vollgelege ist dann erreicht, wenn kein weiteres Ei mehr abgelegt wird (maximale Anzahl Eier erreicht) und das Gelege bebrütet wird. Zur Feststellung des Bebrütungszeitraums ist der Bebrütungsbeginn als der Tag vor der Ablage des letzten Eies definiert. Der Schlupftag ist der erste Lebenstag, am zweiten Lebenstag ist der Nestling einen Tag alt.

Der Schlupftag ist in dieser Studie als der Tag definiert, an dem der erste Jungvogel ausschlüpft. Der Tag lässt sich meistens gut aus dem Alter der Jungvögel abschätzen, zur Berechnung von Nestlingszeit und Brutdauer wurden ausschließlich beobachtete Schlupftage (z.B. noch nicht alle Jungvögel geschlüpft, Jungvogel hat kaum mehr Masse als Ei) herangezogen. Die Erfassung des Ausfliegetages gelang nur in wenigen Ausnahmefällen, weil dazu Kontrollen am Folgetag nötig sind. In den meisten Fällen wurde nach der Beringung der Jungvögel (ungefähr nach 2/3 der Nestlingszeit) nur noch eine Kontrolle durchgeführt, um die Nestparameter zu erfassen. Wenn nicht eindeutige Spuren (Zerstörung des Nestes, Blut, Teile toter Jungvögel) auf Prädation hinwiesen, wurde das Nest als erfolgreich bewertet.

Der Schlupferfolg bezeichnet in dieser Studie den Anteil erfolgreich ausgeschlüpfter Jungvögel an der Anzahl der Eier aller Vollgelege. Die Anzahl der ausgeflogenen Jungvögel je Nest wird als Ausfliegeerfolg bezeichnet (bei BAIRLEIN (1996a) wird der Bezug zur Gesamtsumme aller abgelegten Eier hergestellt).

Von besonderem Interesse bei der Erfassung brutbiologischer Daten ist der Nesterfolg. Nester mit mindestens einem flügge gewordenen Jungvogel werden als erfolgreich betrachtet und der Anteil erfolgreicher Nester, zu den insgesamt mit Eiern gefundenen Nestern, beschreibt den Nesterfolg (BAIRLEIN 1996a).

Zur Berechnung des Nesterfolgs wurde zusätzlich nach der Methode von MAYFIELD (1961) die tägliche Überlebensrate von Nestern aus der Gesamtzahl der kontrollierten Nesttage 
ermittelt. Ich habe dabei auf die urprüngliche Art der Berechnung zurückgegriffen, weil die Vorschläge zur Vereinfachung von MAYFIELD (1975) eine Bewertung der Resultate erschweren (Dow 1978). Die Vorgehensweise nach GREEN (1977) konnte in dieser Studie ebensowenig Verwendung finden wie eine Alternative nach JOHNSON (1979), weil erstens die Intervalle zwischen den Kontrollen zu groß waren und zweitens keine durchgehende Unterscheidung zwischen Lege-, Bebrütungs- und Nestlingsphase möglich war. Für diesen Fall empfiehlt JOHNSON (1979) auch die Verwendung der Methode von MAYFIELD (1961).

Für jede Art wird die Summe der Beobachtungstage (Expositionszeit 'EXP') über die Gesamtzahl der kontrollierten Nester gebildet. Die Anzahl der Nester, die nicht erfolgreich sind (L für lost), teilt man durch die Summe der Beobachtungstage und erhält die tägliche Mortalitätswahrscheinlichkeit $\left(\mathrm{MORT}_{\mathrm{d}}\right)$ :

$$
M O R T_{d}=\frac{L}{E X P}
$$

Die tägliche Überlebenswahrscheinlichkeit $\left(\mathrm{SURV}_{\mathrm{d}}\right)$ ergibt sich damit aus:

$$
S U R V_{d}=1-M O R T_{d}
$$

Die Gesamt-Überlebenswahrscheinlichkeit $\left(\mathrm{SURV}_{\mathrm{t}}\right)$ berechnet sich aus der täglichen Überlebenswahrscheinlichkeit 'hoch' der Anzahl der Tage aus Brut- und Nestlingsphase (ND für Nestdauer, nest duration) der Art:

$$
S U R V_{t}=\left(S U R V_{d}^{N D}\right) \bullet 100
$$

Durch die Faktorisierung werden Prozentwerte erhalten, die den Nesterfolg beschreiben. Die jeweils verwendete Nestdauer für die behandelten Arten ist Tab. $31 \mathrm{zu}$ entnehmen.

Die Nestdauer ist die Summe aus der Bebrütungszeit und der Nestlingszeit und bezieht die Tage der Legephase nicht mit ein, weil diese von der Anzahl der jeweils im Gelege enthaltenen Eier abhängt. Die Zeitspanne der Bebrütungsphase wurde soweit möglich aus den eigenen Beobachtungen errechnet, die Angaben zur Nestlingszeit der Literatur (BEZzEL 1993) entnommen.

\subsection{Populationsentwicklung: Berechnung der Populationsgröße im folgenden Jahr}

Mit der Erfassung des Ausfliegeerfolgs in Hecken lässt sich für die betreffenden Arten die Populationsgröße $\boldsymbol{P}$ für das Folgejahr $\boldsymbol{t}+\boldsymbol{1}$ abschätzen, unter der Annahme dass Immigration und Emigration in einem ausgeglichenen Verhältnis zueinander stehen. Dazu wird die Brutpopulation eines Jahres $\boldsymbol{P}_{\boldsymbol{t}}$ unter Berücksichtigung der Mortalität adulter Individuen $\left(\boldsymbol{M}_{\boldsymbol{a d}}\right)$ 
mit der Summe der Jungvögel $\boldsymbol{J}$ addiert. Der in $\boldsymbol{J}$ ausgedrückte Ausfliegeerfolg wird auf die Populationsgröße $\boldsymbol{P}_{\boldsymbol{t}}$ bezogen und die Sterblichkeit der Jungvögel bis zur Brutzeit im folgenden Jahr wird mit $\boldsymbol{M}_{\boldsymbol{j} \boldsymbol{u}}$ berücksichtigt. Um rechnerisch auf eine Anzahl von Brutpaaren zu kommen, muss die Summe der Jungvögel durch 2 dividiert werden. Unter Berücksichtigung der Brutversuche $\boldsymbol{B}$ der jeweiligen Arten ergibt sich folgende Formel zur Abschätzung der Populationsgröße im Folgejahr $\boldsymbol{P}_{\boldsymbol{t}+\boldsymbol{1}}$ :

$$
P_{t+1}=P_{t} \bullet\left(1-M_{a d}\right)+\frac{P_{t} \bullet J \bullet\left(1-M_{j u v}\right)}{2} \bullet B
$$

mit $\mathrm{P}=$ Populationsgröße (Anzahl Brutpaare; $\mathrm{t}+1=$ Folgejahr $)$;

$\mathrm{M}=$ Mortalität für Adulte (ad: $>1 \mathrm{Jahr}$ ) und Juvenile (juv: $<1 \mathrm{Jahr})$;

$\mathrm{J}=$ Anzahl flügger Jungvögel pro Nest, Ausfliegeerfolg

$\mathrm{B}=$ Anzahl Bruten pro Jahr

Zur besseren Vergleichbarkeit bietet es sich an, eine Populationsgröße von 100 anzunehmen, um die relative Populationsgröße für ein Folgejahr als Prozentwert zum Vorjahr anzugeben. Im praktischen Gebrauch für ein explizit bearbeitetes Gebiet ist die Verwendung tatsächlicher Bestandszahlen vorzuziehen.

\subsection{Ursachen für Brutverluste}

Prädation an kontrollierten Nestern wurde nur als solche erfasst, wenn diese in ihrer Konsequenz zu einem Totalverlust des Nestes führte. Der Fund eines Nestes mit Gelege oder Jungvögeln lässt artspezifisch die Berechnung oder eine Abschätzung des Ausflugtages zu. Wenn das Nest vor dem errechneten Ausfliegetag leer gefunden wurde, habe ich Prädation als Verlustursache angenommen.

Es ist bekannt, dass Prädation nicht immer zum Totalverlust von Nestern führen muss (HALUPKA 1998, HAuBER 1998). Das Verschwinden einzelner Eier oder Jungvögel kann aber viele Ursachen haben, z. B. Entfernung einzelner unbefruchteter Eier, Ei aus dem Nest gefallen, Jungvogel schwächlich, krank oder verhungert und von den Eltern beseitigt u.v.m.. Die Anzahl von Nestern, die durch Prädation Verluste hatten, ist daher nicht genau zu quantifizieren, so dass in dieser Studie nur Totalverluste von Nestern als Prädationsereignisse registriert wurden.

In mehreren Fällen und bei verschiedenen Arten (Amsel, Singdrossel, Goldammer und Heckenbraunelle) kontrollierte ich Nester in der Legephase an aufeinander folgenden Tagen und stellte fest, dass Nester mit 2-4 Eiern am Folgetag ausgeraubt waren, den Tag darauf jedoch wieder ein Ei enthielten, das dann über mehrere Tage im Nest verblieb (in einem Singdrosselnest über fünf Wochen). Auch diese Nester wurden als ausgeraubt erfasst. 
Vermutlich konnte das ausgeraubte Weibchen unter Legedruck ein weiteres Ei nur noch in das alte Nest ablegen, gab dieses aber aufgrund des vorherigen Verlusts auf. In einem Fall legte eine Heckenbraunelle nochmals drei Eier in ein bereits ausgeraubtes Nest. In all diesen Fällen wurde jeweils nur ein Nest und ein Prädationsereignis für die Analysen benutzt.

Mahd wurde als Verlustursache registriert, wenn in der Nestkontrolle vor der landschaftspflegerischen Maßnahme 'Mahd von Randstreifen, Gräben und gehölzbegleitender Krautvegetation' noch ein bebrütetes Nest kontrolliert wurde. Auch für Nester, die aufgrund der Mahd aufgegeben wurden, ohne vollkommen zerstört worden zu sein ('freigemähte Nester'), wurde Mahd als Verlustursache angenommen.

Verluste durch Störungen sind, außer durch Dauerbeobachtung oder selbst verursachte Störungen, nicht eindeutig als Verlustursache von Bruten zu bestimmen. In dieser Arbeit wurden äußerlich unversehrte Gelege als durch Störungseinflüsse aufgegeben beurteilt, wenn keine anderen Faktoren für das Verlassen des Geleges (z.B. Witterung, Nest mit Parasitenbefall) ersichtlich waren. So wurde z.B. ein Singdrosselnest an einem Hochsitz wahrscheinlich durch die Benutzung des Hochsitzes aufgegeben, ein Goldammernest an der Rückseite einer viel benutzten Bank wurde nach längerer Bebrütung verlassen. Es ist nicht auszuschließen, dass Störungen auch natürlich bedingt sein können (z.B. Wildwechsel durch eine Hecke, Wildschweine, ungünstig überwachsende Vegetation), in der Regel lag die Vermutung einer Störung durch menschliche Aktivitäten am nächsten.

Zum Vergleich relativer Häufigkeiten von gefundenen und ausgeraubten Nestern wurde der Z-Test (SACHS 1992) angewandt.

\subsection{Ermittlung der Prädatoren an den Kunstnestern}

Das Spektrum der Nesträuber an Kunstnestern sollte qualitativ und quantitativ erfasst werden. Dazu wurden Eier der Zwergwachtel (Coturnix japonica) in Kunstnestern ausgebracht und mit einem Fotoapparat gekoppelt, der bei Entnahme des Eis auslöste. Das Ei wurde dabei in einer kleinen Halterung aus Draht auf dem Nestboden befestigt. Eine Seite der Halterung hatte eine kleine Feststellschraube, mit der das Ei auf den Auslöser gedrückt wurde. Die zur Erfassung von nachtaktiven Tieren mit einem Blitz ausgestatteten Kameras wurden in 1,5 bis $2 \mathrm{~m}$ Entfernung zum Nest auf einer Stange positioniert. Die Energieversorgung erfolgte durch externe 6 V-Akkus, um die Blitzbereitschaft für $24 \mathrm{~h}$ sicherzustellen. Die Aufstellung der Kameras erfolgte zufällig über die bearbeiteten Hecken verteilt. Die Standorte der Nester entsprachen denen zur Analyse von Prädationsmustern an Kunstnestern (etwa 0,5 m Höhe und $0,5 \mathrm{~m}$ in das Heckengehölz eingerückt). Zweige im Sichtfeld zur Kamera wurden 
entfernt. Die Standorte der fünf unabhängig voneinander eingesetzten Kameras wurden nach jedem Foto, spätestens jedoch nach einer Woche gewechselt. Kontrollen und Akkuwechsel erfolgten täglich. Jedes der fünf Systeme wurde zwischen April bis Juli 1999 und 2000 monatlich etwa 20 Tage eingesetzt.

Zum Vergleich zweier unabhängiger Stichproben (Prädationsereignisse von Eichelhäher und Elster) mit kleiner Stichprobengröße, wurde der $\mathrm{Chi}^{2}$ Exact-Test mit StatXact 4.0 verwendet.

\subsection{Prädationsmuster}

Anhand von Ausraubraten an Kunstnestern sollte herausgefunden werden, welche Neststandorte ein erhöhtes Prädationsrisiko bedingen. Zu Beginn der Hauptbrutzeit (4.-8. Mai 1998), zwei Wochen später (18.-22. Mai) und zwischen dem 16.-20. Juni wurden zweimal 377 Kunstnester an Hecken ausgebracht, im Juni folgten nochmals 162 Nester. Die zweite Ausbringung ist eine Replikation der ersten, im Juni wurden andere Standorte für die Kunstnester ausgewählt.

Die benutzen Nester waren selbstgefertigte Drahtkörbchen aus wabenförmigem, grünem Zaundraht mit $20 \mathrm{~mm}$ Maschenweite, die jeweils an einer 0,6 mm starken Betonstahlstange befestigt und in der Höhe verstellbar waren (Abb. 23). In die Körbchen wurde feines Stroh eingedreht und jeweils ein Wachtelei eingelegt. Die Eier der Zwergwachtel Coturnix japonica wurden von einem Geflügelzüchter bezogen. Der ockerfarbene Grund mit braun-schwarzer Fleckung ließ die Eier in den Nestern unauffällig erscheinen. Die Maße der Wachteleier lagen bei $350 \mathrm{~mm}(\min 315-\max 390 \mathrm{~mm}, \mathrm{~S}=0,2) * 267 \mathrm{~mm}(\min 250-\max 285 \mathrm{~mm}, \mathrm{~S}=0,1)$ für 24 ausgemessene Eier.

Die Ausbringung der Nester erfolgte in Abständen von jeweils 10 m, auf beiden Heckenseiten gegenseitig um $5 \mathrm{~m}$ versetzt. Jedes Nest konnte durch die Stange unabhängig von umgebenden Strukturen in jeweils etwa 0,5 m Höhe und $0,5 \mathrm{~m}$ vom Gehölzrand eingerückt an der Hecke plaziert werden. Damit sollte in etwa der Nestortwahl von Grasmückenarten, Heckenbraunelle und Goldammer entsprochen werden. Mit der systematischen Ausbringung der Nester entlang der Hecken ergab sich eine zufällige Auswahl von Neststandorten. 


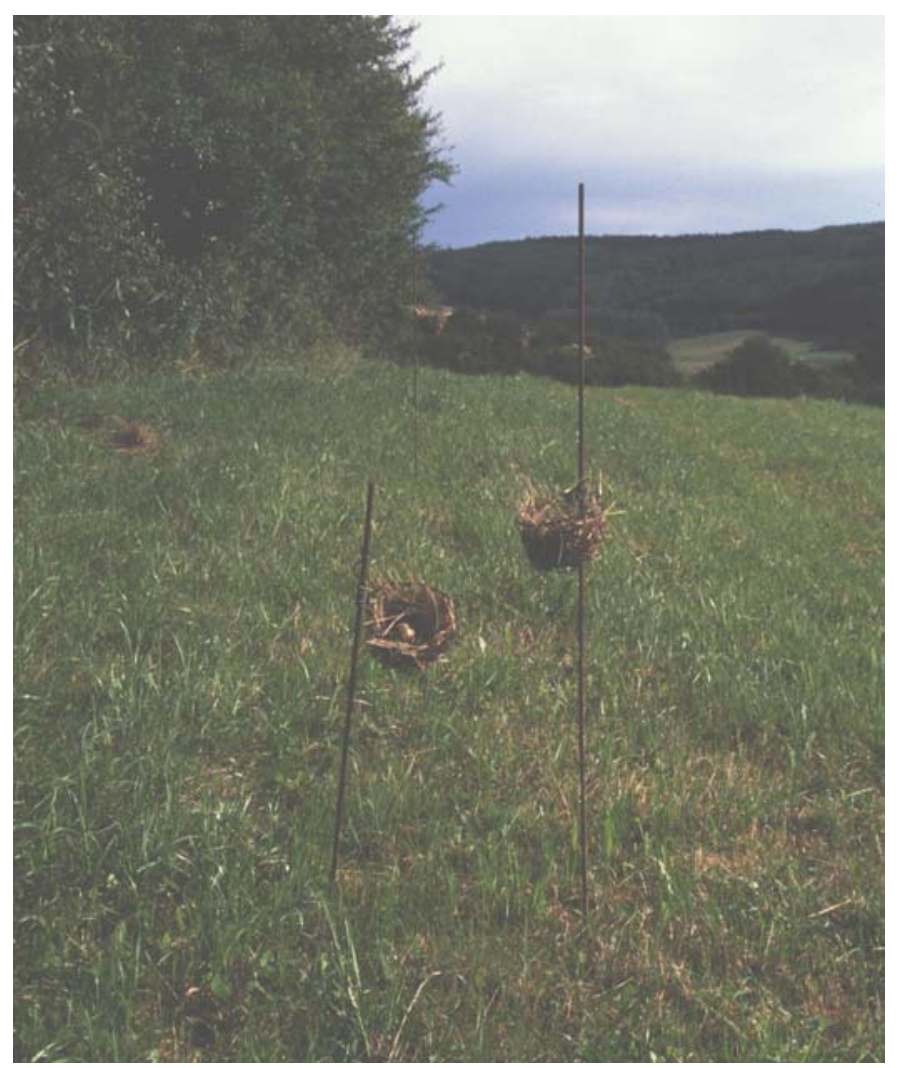

Abb. 23: Zur Anschauung freigestellte Kunstnester vor der Plazierung in der Hecke

Die Nester wurden jeweils einmal nach drei Tagen und dann abschließend nach sieben Tagen kontrolliert. Erhaltene Eier wurden nach Ablauf der Woche wieder eingesammelt.

Mit diesem Ansatz sollten zwei Fragen behandelt werden, erstens: Gibt es Unterschiede der Nestverlustraten in Abhängigkeit des Fortschritts der Brutzeit? Zweitens: Welche Neststandortparameter bieten den besten Schutz vor Nesträubern?

Zum Vergleich relativer Häufigkeiten wurde der Z-Test (SACHS 1992) angewendet, die Analyse der wichtigsten Neststandortvariablen erfolgte anhand einer schrittweise vorwärts durchgeführten binären Logistischen Regression mit SPSS Version 10.07. Das Aufnahmekriterium für Variablen wurde auf $p<0,05$, für die Eliminierung einer Variablen aus dem Modell wurde mit $\mathrm{p}<0,1$ festgelegt. In die Analyse wurden 496 Nester einbezogen, für die folgende Parameter jeweils am Neststandort erhoben wurden:

Heckenhöhe und Heckenbreite [m, 0,5 m Messgenauigkeit]

Heckendichte [vier Klassen, $1 \mathrm{~m}^{2}$ großer Karton mit Rot-Weiß-Karo zur Abschätzung der Geästdichte in Brusthöhe zwischen Heckenmitte und -rand]

Nestsichtbarkeit von oben und von den Seiten [vier Klassen, $1 \mathrm{dm}^{2}$ Karton mit Rot-WeißKaro à $1 \mathrm{~cm}^{2}$ zur Abschätzung von vier Sichtbarkeitsklassen jeweils $0,5 \mathrm{~m}$ über bzw. von vier Seiten des Nests. Die Nestsichtbarkeit von der Seite wurde aus vier Werten gemittelt und einer der vier Klassen zugeordnet.] 
Vegetation um das Nest [drei Klassen zur Einschätzung der Wahrnehmbarkeit des Kunstnestes vom Heckenrand in Abhängigkeit zur Vegetation vor dem Nest; Nest fast frei von umgebender Vegetation bis Nest fast vollständig von Vegetation verdeckt.]

Saum vorhanden oder nicht [binär, Krautsaum wurde als vorhanden angesehen, wenn Feldnutzung oder Wegbau nicht unmittelbar an die Gehölzstruktur der Hecke heranreichten.]

Saumbreite und Saumhöhe [m, Breite des Krautsaums wurde zwischen Gehölzrand der Hecke in 0,5 m Höhe und dem Beginn der Nutzflächen gemessen, die Höhe wurde in der Mitte des Krautsaums gemessen, als Entfernung zwischen einer auf der Vegetation aufliegenden Pappscheibe und dem Boden. Die mittig von einem Stab geführte Pappfläche wurde zu diesem Zweck auf die Vegetation fallen gelassen. Messpunkte waren Bodenoberfläche und Höhe der Pappscheibe am Führungsstab.]

Exposition [acht Himmelsrichtungen wurden jeweils binär erfasst.]

Die Erfassung und Beschreibung aller Variablen in Bezug auf das einzelne Nest setzt voraus, dass keine übergeordneten Ereignisse auf die Ausraubwahrscheinlichkeit eines Nestes wirken. Es ist aber durchaus realistisch anzunehmen, dass bestimmte Nesträuber nur in bestimmten Hecken vorkommen und darin dann sehr viele Nester finden und in anderen Hecken gar nicht danach suchen. Um zu überprüfen, ob Nester 'heckenweise' ausgeraubt werden, wurde überprüft, ob ein Nest mit höherer Wahrscheinlichkeit ausgeraubt wird, wenn das Nachbarnest ausgeraubt wurde (Logistische Regression, SPSS 10.07). Dazu wurde mit einer neuen unabhängigen Variablen 'Nachbar' beschrieben, ob eines der unmittelbar benachbarten Nester ausgeraubt worden ist.

Um den Einfluss von Heckenstrukturen, Landschaftsparametern und Auswirkungen von Nutzungsformen auf die Prädation von Kunstnestern zu analysieren, wurden 1999 in 41 Hecken jeweils sechs Nester (drei je Seite) für eine Woche exponiert. Der Abstand zwischen den Nestern betrug $25 \mathrm{~m}$, ansonsten erfolgte die Positionierung der Kunstnester wie im Vorjahr. Mit einer Logistischen Regression wurden die wichtigsten Variablen zur Klassifizierung extrahiert, die Irrtumswahrscheinlichkeit für eine aufzunehmende Kriteriumsvariable wurde auf $\mathrm{p}<0,1$ heraufgesetzt, um mehr als nur eine unabhängige Variable in das Modell aufnehmen zu können. Die abhängige Variable wurde binär beschrieben, als Hecke komplett ausgeraubt oder nicht. 
Im Einzelnen wurden für 41 Hecken folgende 23 Variablen aufgenommen:

Neben den zur Strukturkartierung aufgenommenen Heckenstrukturen Länge, Breite, Krautsaum, Überhälter und Heckendichte (Beschreibung siehe Abschnitt 10.1) wurden

Kern [m; Breite des von Bäumen überwachsenen Bereichs der Hecke],

Mantel [m; Breite der Strauchausprägung, Bereich zwischen Kern und Krautsaum] und

Doppelhecke [binär, Hecke doppelreihig wegsäumend ausgeprägt oder nicht]

aufgenommen. Alle metrisch erfassten Heckenstrukturen wurden an den Neststandorten direkt erhoben (keine Luftbildauswertung).

Neben den zur Strukturkartierung aufgenommenen Landschaftsparametern Siedlungsnähe, Waldnähe, Wegedichte, Heckenabundanz, Heckenverbund, Acker und Brache (Beschreibung siehe Abschnitt 10.1) wurden folgende weitere Variablen aufgenommen:

Wiese, Weide oder Wald [\%; an Hecke grenzende Flächennutzung],

Straße, Weg, Garten, Gebäude oder Graben [binär; an Hecke grenzend vorhanden oder nicht].

Um eine Vorauswahl zu treffen wurden nur Variablen mit $p<0,4$ zur Aufnahme in das Modell ausgewählt. Mit den verbliebenen 11 Variablen (Breite mit p=0,286, Kern 0,284, Mantel 0,071, Heckendichte 0,177, Doppelhecke 0,188, Siedlungsnähe 0,060, Waldnähe 0,090, Wegedichte 0,029, Wald 0,074, Straße 0,240, Weg 0,272) wurde eine Logistische Regression (binär, schrittweise vorwärts und zur Überprüfung der Validität schrittweise rückwärts) durchgeführt. 


\section{Ergebnisse}

\subsection{Brutvogelbestand und Heckenstrukturen}

In 30 Hecken wurden von 1998 bis 200041 Brutvogelarten nachgewiesen (Tab. 20). Auf 5.845 m Heckenlänge wurden durchschnittlich 514 Reviere im Jahr erfasst (min: 500; max: 530), das entspricht einer mittleren Revierdichte von 87,9 BP/km (min: 85,5; max: 90,7), mit einer mittleren Entfernung von 11,5 m Heckenstrecke zwischen den Revieren.

Über alle Hecken betrachtet sind die summierten Anzahlen der Brutpaare über die drei Untersuchungsjahre sehr ausgeglichen und schwanken um weniger als $5 \%$. In einzelnen Hecken treten allerdings zum Teil beträchtliche Brutvogelbestandsveränderungen auf. In Hecke 51 wurden 1998 16, in den Folgejahren nur noch 7 bzw. 8 Reviere festgestellt. Auch die Artenzahl ging von 12 auf 7 zurück. Bei dieser Hecke handelt es sich um eine waldnahe Feldhecke mit einigen überragenden Pappeln. Dazwischen und darunter stehende, sehr alte Schlehen wurden im Herbst 1998 an einigen Stellen zurückgeschnitten und das Schnittgut entfernt. Obwohl der Pflegeeingriff unter Beibehaltung der Heckenstruktur erfolgte, war in den Folgejahren ein Rückgang oder Ausbleiben von Goldammer, Heckenbraunelle, Gartengrasmücke, Kohlmeise, Rotkehlchen und Feldschwirl festzustellen.

In Hecke 67 gab es von 1998 auf 1999 einen Anstieg von 12 auf 19 Brutpaare, die im Jahr 2000 wieder auf 10 BP zurückgingen, ohne dass Veränderungen an der Gehölzstruktur der Hecke vorgenommen wurden. Ein Dickicht aus Brombeeren und Brennesseln wurde 1999 nicht zurückgeschnitten, was Arten wie Heckenbraunelle, Gartengrasmücke, Sumpfrohrsänger und Feldschwirl zu Gute kam.

In Hecke 44 verschwanden mit Singdrossel, Zilpzalp, Buchfink und Turteltaube im Jahr 2000 vier Arten, die in vorherigen Jahren festgestellt werden konnten. In der Hecke wurde im Frühjahr 2000, unter Einsatz von schwerem Gerät, ein Hochsitz aufgebaut, was möglicherweise zu Störungen während der Phase der Reviergründung geführt hat.

Bemerkenswert ist die Zunahme der Summe der Brutpaare in Hecke 54, die mit einer stetig steigenden Nutzung des (in allen Untersuchungsjahren konstanten) Nistkastenangebots durch Feldsperlinge einher ging. In Hecke 97 ist die Abnahme von Kohlmeise und Feldsperling auf die Entfernung von Nistkästen in der Hecke zurückzuführen. 
Tab. 20: Anzahlen der Reviere von Brutvögeln in Hecken, die von 1998 bis 2000 kartiert wurden. Brutvögel ( $N=41)$ und Hecken $(\mathrm{N}=30)$ sind nach Häufigkeit bzw. Artenzahl sortiert. Unter 'Ort' sind die Untersuchungsgebiete angegeben; mit Ge - Geismar, Ap - Appenrode-Gleichen, Vo - Volkerode, Jü Jühnde und Le - Lemshausen. Unter 'Hecke (Länge) Arten' folgt eine Heckennummer und darunter in Klammern die Länge der betreffenden Hecke sowie die Artenzahl. Alle Angaben einschließlich der Summen der Brutpaare (BP) und der Brutpaare je Art sind Anzahlen ohne Flächenbezug. Mit * gekennzeichnete Arten profitierten von künstlichen Nisthöhlen in einigen (*) Hecken.

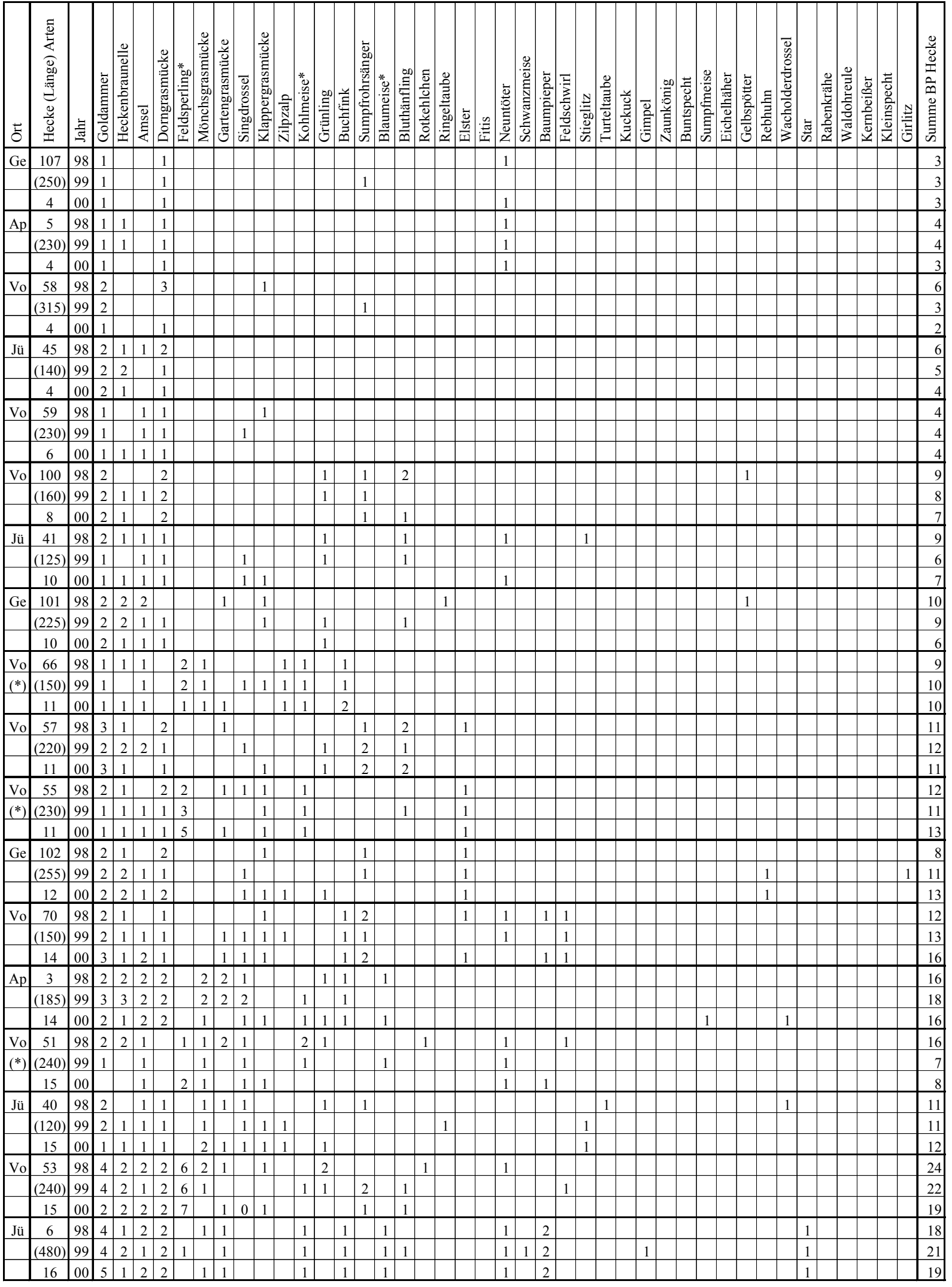


Fortsetzung von Tab. 20: Anzahlen der Reviere von Brutvögeln in Hecken.

\begin{tabular}{|c|c|c|c|c|c|c|c|c|c|c|c|c|c|c|c|c|c|c|c|c|c|c|c|c|c|c|c|c|c|c|c|c|c|c|c|c|c|c|c|c|c|c|c|}
\hline 5 & 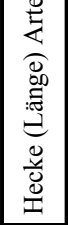 & 责 & 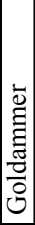 & 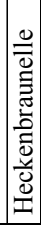 & $\begin{array}{l} \\
\overline{0} \\
0 \\
\text { Z } \\
\end{array}$ & 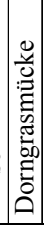 & 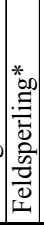 & 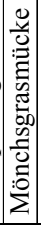 & 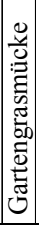 & 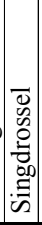 & 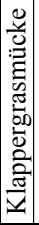 & 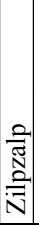 & 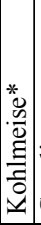 & , & 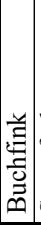 & 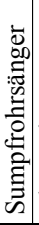 & 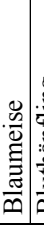 & 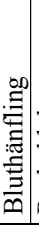 & 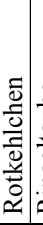 & 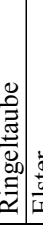 & 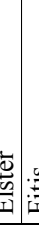 & 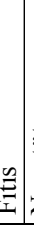 & 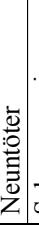 & 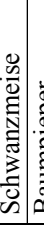 & 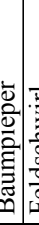 & 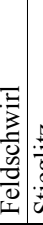 & 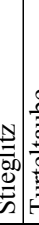 & 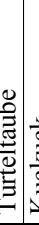 & & & 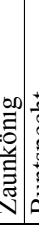 & 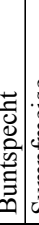 & 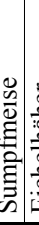 & 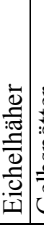 & $\begin{array}{l}\overrightarrow{0} \\
: 0 \\
0 \\
0 \\
0 \\
0 \\
0 \\
0\end{array}$ & & 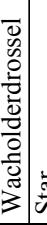 & 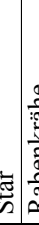 & 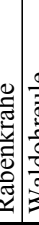 & & 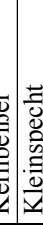 & 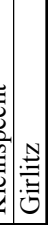 & 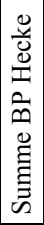 \\
\hline $\mathrm{Vo}_{\mathrm{O}}$ & 56 & 98 & 4 & 2 & 2 & 2 & 3 & & 1 & 1 & 1 & & \begin{tabular}{|l|}
1 \\
\end{tabular} & 1 & & 2 & & 2 & & 1 & 1 & & & & & & & 1 & & & & & & & & & & & & & & & 25 \\
\hline$(*)$ & (230) & 99 & 2 & 2 & 1 & 2 & 4 & & 2 & 1 & 1 & & 1 & 1 & & & 1 & 1 & & & 1 & & & & & & & & & & & & & & & & & & & & & & 20 \\
\hline & 16 & 00 & 2 & 2 & 1 & 2 & 5 & & & 1 & 1 & & & 1 & & & & 1 & & & 1 & & & & & & & & & & & & & & & & & & & & & & 17 \\
\hline Vo & 68 & 98 & 2 & 2 & 1 & & 1 & 1 & 1 & 1 & & 11 & & & & & & & & & & & & & & & 1 & & & & & & & & & & & & & & & & 11 \\
\hline$(*)$ & (110) & 99 & 1 & 2 & 2 & 1 & & 1 & 1 & 1 & 1 & 1 & & 1 & & & & & & & & 1 & & & & & & & & & & & & & & 1 & & & & & & & 14 \\
\hline & 17 & 00 & 1 & 2 & 2 & 2 & & 1 & 2 & 1 & 1 & 1 & & & & 1 & & & 1 & & 1 & & & & & & & & & & & & & & & & & & & & & & 16 \\
\hline Vo & 67 & 98 & 2 & 1 & 1 & & & 1 & 1 & 1 & & 1 & 1 & & & & 1 & & & 1 & & & & & & & & & & 1 & & & & & & & & & & & & & 12 \\
\hline & (125) & 99 & 1 & 2 & 1 & 1 & & 1 & 2 & 1 & 1 & 1 & 1 & & & 1 & 1 & & & & 12 & 2 & & & & 1 & & & & & & & & & & & & & & 1 & & & 19 \\
\hline & 18 & 00 & 1 & 1 & 1 & 1 & & 1 & 1 & 1 & & 1 & 1 & & & & 1 & & & & & & & & & & & & & & & & & & & & & & & & & & 10 \\
\hline $\mathrm{Jü}$ & 44 & 98 & 2 & 1 & 1 & 1 & & 1 & 2 & 1 & & 1 & & & 1 & 2 & & & & & & & & & 1 & & 1 & 1 & & & & & & & 1 & & & & & & & & 17 \\
\hline & $(210)$ & 99 & 2 & 1 & 2 & 1 & & 1 & 2 & 1 & 1 & 1 & & & 1 & & & & & 1 & & & & & & & & 1 & 1 & & & & & 1 & & & & & & & & & 17 \\
\hline & 18 & 00 & 1 & 2 & 1 & 1 & & 1 & 2 & & 1 & & & & & & & & & & & & & & & & & & 1 & & & & & & & & & & & & & & 10 \\
\hline Vo & 69 & 98 & 2 & 1 & 1 & 2 & 2 & 1 & 1 & & 1 & & 1 & & 1 & & 1 & & 1 & & 1 & & & & & & & & 1 & & & & & & & & & & & & & & 17 \\
\hline$(*)$ & $(260)$ & 99 & 2 & 3 & 2 & & 3 & 1 & 1 & 1 & & 1 & 1 & & 1 & & 1 & & 1 & & 1 & 1 & & & & 1 & & & 1 & & & & & & & & & & & & & & 22 \\
\hline & 19 & 00 & 1 & 1 & 1 & 1 & 4 & 2 & 2 & & 1 & 2 & 1 & & 1 & & 1 & & 1 & 1 & & & & & & & & & 1 & & & & & & & & & & & & & & 21 \\
\hline $\mathrm{Ge}$ & 103 & 98 & 4 & 4 & 1 & 2 & & 1 & 2 & 1 & 2 & 2 & & 1 & & & 1 & & & 1 & & 1 & & & & & & & & & & & & & & & & & & & & & 23 \\
\hline & $(320)$ & 99 & 2 & 2 & 2 & 2 & & 2 & 2 & 2 & & & 1 & & & & 1 & & 1 & 1 & & 1 & & 1 & & & & & & & & & 1 & & & & & & & & & & 21 \\
\hline & 18 & 00 & 2 & 2 & 2 & 1 & & 1 & & 2 & 2 & 2 & & 1 & & & & & 1 & & & & & 1 & & & & & & & & & & & & & & & & & & & 17 \\
\hline Vo & 52 & 98 & 2 & 2 & 2 & 1 & 3 & 2 & 2 & 2 & & 1 & 2 & 2 & & & 1 & 1 & 1 & & 1 & & & 1 & & & & & & & & & & & & & & & & & & & 26 \\
\hline$(*)$ & $(255)$ & 99 & 4 & 1 & 2 & 2 & 5 & 2 & 2 & 2 & 1 & 1 & 1 & 1 & & & & & & \begin{tabular}{l|l}
2 & \\
\end{tabular} & 1 & & & & 1 & 1 & & & & & & & & & & 1 & & & & & & & 30 \\
\hline & 22 & 00 & 3 & 3 & 2 & 1 & 8 & 2 & 2 & 2 & 2 & 1 & 2 & 2 & 2 & & & & & 1 & & & & 1 & 1 & & & & & & & & & & & & & & & & & & 35 \\
\hline $\mathrm{Le}$ & 96 & 98 & 4 & 2 & 2 & 3 & & 1 & 1 & 1 & 2 & 2 & 1 & 2 & & & 1 & 2 & 2 & & & 1 & 1 & & & & & & & & & & & & & & & & & & & & 28 \\
\hline & $(290)$ & 99 & 5 & 1 & 2 & 4 & & 1 & 1 & 1 & & 1 & 2 & 2 & 2 & 1 & 1 & 3 & 1 & & & 1 & 1 & & & \begin{tabular}{l|l} 
& \\
\end{tabular} & 1 & & & & & & & & & & & & & & & & 32 \\
\hline & 22 & 00 & 5 & 2 & 2 & 4 & & 1 & 1 & 1 & 4 & 1 & 1 & 2 & & 2 & 1 & 2 & & & & & 1 & & & 1 & 1 & & & & & 1 & & & & & & & & 1 & 1 & & 34 \\
\hline $\mathrm{Vo}$ & 54 & 98 & 3 & 3 & 2 & 2 & 4 & 1 & 1 & 1 & 2 & 1 & 2 & 1 & & & & & 1 & 1 & 1 & & & & & & 1 & & & 1 & & & & & & & & & & & & & 28 \\
\hline$(*)$ & (525) & 99 & 4 & 2 & 3 & 4 & 8 & 1 & 1 & 1 & & 1 & 2 & 1 & 3 & & 1 & & & & 1 & & & 1 & & 2 & & & & 1 & & & & & & & & & & & & & 37 \\
\hline & 22 & 00 & 2 & 2 & 3 & 3 & 12 & 1 & 2 & 1 & 3 & 1 & 1 & 1 & 2 & & & & 1 & 1 & 1 & & & 1 & & & & & & 1 & & & & & & & & & & 1 & & & 40 \\
\hline $\mathrm{Jü}$ & 43 & 98 & 2 & 4 & 3 & & & 3 & 3 & 1 & 2 & 3 & & 1 & 1 & 1 & 1 & & & 1 & & & & & & & & 1 & & 1 & & & & & & & & & & 1 & & & 29 \\
\hline & $(160)$ & 99 & 3 & 3 & 2 & & & 3 & 3 & 1 & 1 & 2 & 1 & & 1 & & 1 & & & 1 & 1 & 1 & & & 1 & & 12 & 2 & 1 & 1 & & 1 & & & & & & & & & & & 31 \\
\hline & 23 & 00 & 3 & 4 & 2 & & & 3 & 3 & 1 & 1 & 2 & 1 & 1 & & & 1 & & & 1 & & & & & & & & 1 & 1 & & & & & & & & & & & & & & 25 \\
\hline $\mathrm{Jü}$ & 42 & 98 & 4 & 2 & 3 & 2 & & 3 & 2 & 3 & 1 & 1 & 1 & & 1 & 2 & 1 & & & 1 & & & & & & & 1 & 1 & & & & & & 1 & 1 & & 1 & & & & & & 32 \\
\hline & $(280)$ & 99 & 5 & 2 & 3 & 2 & & 4 & 2 & 3 & 2 & 3 & 2 & 1 & 2 & & 1 & & & 2 & & 1 & & 1 & 1 & & & 1 & 1 & & & 1 & 1 & & & & 1 & & & & & & 42 \\
\hline & 28 & 00 & 5 & 2 & 3 & 2 & & 4 & 1 & 2 & & 2 & & 1 & & & & & & 2 & & & & 1 & & 1 & & 1 & 1 & & & & & 1 & & & & & & 1 & & & 30 \\
\hline $\mathrm{Le}$ & 97 & 98 & 4 & 4 & 5 & 1 & 6 & 5 & 3 & 4 & 1 & 6 & 5 & 1 & 2 & 2 & 2 & & 5 & 1 & & 3 & & 1 & & & & & & 1 & 2 & 1 & 1 & 1 & & & & 1 & & & & & 68 \\
\hline & (575) & 99 & 4 & 4 & 7 & 2 & 2 & 7 & 3 & 4 & & 5 & 5 & & 5 & \begin{tabular}{|l|} 
\\
\end{tabular} & 1 & 1 & 3 & 1 & & 3 & & 2 & & & 2 & & 1 & 1 & 3 & 1 & 1 & 1 & & & & & 1 & & & & 71 \\
\hline & 31 & 00 & 4 & 5 & 7 & 1 & 2 & 9 & 5 & 5 & 4 & 4 & 2 & 3 & 5 & 1 & 1 & & 3 & 1 & & 3 & & 3 & & & & & 1 & 1 & 3 & 1 & 1 & 1 & 1 & & & & 2 & & 1 & & 80 \\
\hline & & & & $\stackrel{ \pm}{m}$ & 기 & $\vec{\beth}$ & $\stackrel{0}{ }$ & \& & $\infty$ & $m$ & $\overline{6}$ & 요 & $n$ & $\because$ & J & $\hat{m}$ & ¿े: & $\stackrel{\infty}{\sim}$ & 2 & $\therefore$ & & 2 & 24 & $\because=$ & - & $\cong$ 工 & -1 & $=$ & -17 & $\underline{\underline{\theta}}$ & $\infty$ & 6 & o & 0 & & & & & & & & -1 & 号 \\
\hline
\end{tabular}

Die jährlichen Veränderungen der Revierzahlen einer Art innerhalb einer Hecke lag bei ein oder zwei Revieren je Hecke. Lediglich Feldsperling und Kohlmeise wiesen stärkere Abweichungen je Hecke und Jahr auf (s.o.). Um zu überprüfen, ob Bestandsveränderungen in der Summe aller Hecken für Arten festzustellen sind, wurde in Tab. 21 für jede Art die Summe aller erfassten Brutpaare für die drei Untersuchungsjahre zusammengestellt.

Bei Heckenbraunelle, Dorngrasmücke und Zilpzalp waren die Reviersummen nahezu konstant. Auch Goldammer, Amsel, Mönchs- und Gartengrasmücke zeigten unter den häufigsten 14 Arten (über 30 Reviere festgestellt) mit weniger als $20 \%$ geringe Schwankungen zwischen den Jahren. Abweichungen zwischen 40-60\% waren bei 
Feldsperling, Singdrossel, Sumpfrohrsänger, Grün- und Kohlmeise festzustellen. Die auffälligsten Bestandsveränderungen hatten Buchfink und Klappergrasmücke (Tab. 21).

Tab. 21: Entwicklung der Revierzahlen aller Brutvögel über alle 30 Hecken.

\begin{tabular}{|l|r|r|r|}
\hline \multicolumn{1}{|c|}{ Art } & 1998 & 1999 & 2000 \\
\hline Goldammer & 72 & 69 & 61 \\
Heckenbraunelle & 45 & 45 & 44 \\
Amsel & 38 & 45 & 43 \\
Dorngrasmücke & 41 & 40 & 40 \\
Feldsperling & 30 & 34 & 46 \\
Mönchsgrasmücke & 28 & 30 & 32 \\
Gartengrasmücke & 30 & 26 & 27 \\
Singdrossel & 21 & 29 & 23 \\
Klappergrasmücke & 19 & 13 & 29 \\
Zilpzalp & 20 & 20 & 20 \\
Kohlmeise & 19 & 23 & 13 \\
Grünling & 16 & 12 & 17 \\
Buchfink & 10 & 19 & 15 \\
Sumpfrohrsänger & 15 & 12 & 10 \\
Blaumeise & 11 & 11 & 7 \\
Bluthänfling & 10 & 11 & 7 \\
Rotkehlchen & 12 & 6 & 7 \\
Ringeltaube & 8 & 9 & 7 \\
Elster & 8 & 8 & 6 \\
Fitis & 5 & 11 & 3 \\
Neuntöter & 8 & 5 & 6 \\
\hline
\end{tabular}

\begin{tabular}{|l|r|r|r|}
\hline \multicolumn{1}{|c|}{ Art } & 1998 & 1999 & 2000 \\
\hline Schwanzmeise & 2 & 6 & 7 \\
Baumpieper & 4 & 5 & 5 \\
Feldschwirl & 2 & 8 & 3 \\
Stieglitz & 5 & 5 & 2 \\
Turteltaube & 5 & 4 & 2 \\
Kuckuck & 1 & 5 & 5 \\
Gimpel & 4 & 4 & 2 \\
Zaunkönig & 2 & 3 & 3 \\
Buntspecht & 1 & 3 & 2 \\
Sumpfmeise & 1 & 3 & 2 \\
Eichelhäher & 2 & 2 & 2 \\
Gelbspötter & 4 & 0 & 1 \\
Rebhuhn & 0 & 3 & 1 \\
Wacholderdrossel & 2 & 1 & 1 \\
Star & 2 & 1 & 1 \\
Rabenkrähe & 0 & 1 & 2 \\
Waldohreule & 0 & 1 & 2 \\
Kernbeißer & 1 & 0 & 1 \\
Kleinspecht & 0 & 0 & 1 \\
Girlitz & 0 & 1 & 0 \\
\hline gesamt & 466 & 478 & 463 \\
\hline
\end{tabular}

Es stellt sich die Frage, ob Veränderungen um ein oder zwei Brutpaare in einer Hecke als geringfügig einzuschätzen sind oder ob bei der begrenzten räumlichen Ausdehnung der untersuchten Hecken kaum größere Bestandsveränderungen zu erwarten sind. Meine Annahme war, dass Hecken als anthropogen geformte Lebensräume nur als ersatzweise für qualitativ bessere Habitate von Populationsüberschüssen dienen und infolgedessen keine hohe Besiedlungskonstanz durch Vögel aufweisen. Die Befunde in Tab. 21 zeigen dies für einige Arten (z.B. Klappergrasmücke, Kohlmeise, Feldsperling, Rotkehlchen, Fitis). In Tab. 22 wurde das Vorkommen der Arten mit der Dauerhaftigkeit der Besiedlung über die Jahre in Beziehung gesetzt. Im Sinne der Hypothese ungünstig wurde definiert, dass nur Bestandsveränderungen erfasst wurden, die zur Besiedlung oder Aufgabe einer Hecke als Brutort durch eine Art führten. 
Tab. 22: Anzahl besiedelter Hecken (von N=30), unterschieden nach alljährlicher (über drei Jahre) und zeitweiliger Nutzung als Brutrevier. Unter C und D sind Anteile zeitweise besiedelter Hecken bzw. Reviere angegeben.

\begin{tabular}{|c|c|c|c|c|}
\hline Art & $\begin{array}{c}\mathrm{N} \text { besiedelter } \\
\text { Hecken }\end{array}$ & $\begin{array}{c}\mathrm{N} \text { alljährlich } \\
\text { besiedelt }\end{array}$ & $\begin{array}{c}\% \text { Hecken } \\
\text { zeitweilig bes. }\end{array}$ & $\begin{array}{c}\% \text { Reviere } \\
\text { zeitweilig bes. }\end{array}$ \\
\hline Goldammer & 30 & 29 & 3 & 2 \\
\hline Heckenbraunelle & 28 & 21 & 25 & 10 \\
\hline Dorngrasmücke & 27 & 22 & 19 & 11 \\
\hline Amsel & 27 & 21 & 22 & 9 \\
\hline Klappergrasmücke & 25 & 4 & 84 & 74 \\
\hline Singdrossel & 22 & 13 & 41 & 18 \\
\hline Gartengrasmücke & 21 & 11 & 48 & 24 \\
\hline Grünling & 18 & 4 & 78 & 62 \\
\hline Mönchsgrasmücke & 17 & 15 & 12 & 6 \\
\hline Kohlmeise & 16 & 9 & 44 & 26 \\
\hline Sumpfrohrsänger & 16 & 4 & 75 & 49 \\
\hline Zilpzalp & 15 & 9 & 40 & 22 \\
\hline Blaumeise & 13 & 6 & 54 & 35 \\
\hline Buchfink & 12 & 6 & 50 & 36 \\
\hline Ringeltaube & 12 & 3 & 75 & 54 \\
\hline Feldsperling & 11 & 8 & 27 & 5 \\
\hline Elster & 11 & 4 & 64 & 46 \\
\hline Bluthänfling & 11 & 3 & 73 & 43 \\
\hline Rotkehlchen & 9 & 2 & 78 & 44 \\
\hline Feldschwirl & 9 & 1 & 89 & 77 \\
\hline Stieglitz & 9 & 0 & 100 & 100 \\
\hline Neuntöter & 8 & 4 & 50 & 37 \\
\hline Fitis & 8 & 1 & 88 & 53 \\
\hline Baumpieper & 7 & 1 & 86 & 57 \\
\hline Schwanzmeise & 6 & 1 & 83 & 60 \\
\hline Gimpel & 5 & 2 & 60 & 40 \\
\hline Turteltaube & 5 & 2 & 60 & 27 \\
\hline Gelbspötter & 5 & 0 & 100 & 100 \\
\hline Buntspecht & 4 & 1 & 75 & 50 \\
\hline Sumpfmeise & 4 & 1 & 75 & 50 \\
\hline Kuckuck & 4 & 1 & 75 & 72 \\
\hline Eichelhäher & 3 & 1 & 67 & 50 \\
\hline Rebhuhn & 3 & 0 & 100 & 100 \\
\hline Wacholderdrossel & 3 & 0 & 100 & 100 \\
\hline Waldohreule & 3 & 0 & 100 & 100 \\
\hline Star & 2 & 1 & 50 & 25 \\
\hline Kernbeißer & 2 & 0 & 100 & 100 \\
\hline Zaunkönig & 1 & 1 & 0 & 0 \\
\hline Rabenkrähe & 1 & 0 & 100 & 100 \\
\hline Kleinspecht & 1 & 0 & 100 & 100 \\
\hline Girlitz & 1 & 0 & 100 & 100 \\
\hline gesan & 432 & 212 & 49 & 24 \\
\hline
\end{tabular}

Über $70 \%$ der Arten nutzen die meisten (50\% und mehr) der von ihnen 'besiedelbaren' Hecken nur in bestimmten Jahren. Besiedelbare Hecken sind solche, die in mindestens einem 
der drei Untersuchungsjahre als Revierstandort genutzt worden sind. Unabhängig von der Einschätzung darüber, ob die Veränderung von ein oder zwei Brutpaaren pro Jahr eine relativ große oder geringe Fluktuation der Revierbesetzung darstellt, zeigt die Unterscheidung von dauerhaft und zeitweilig besetzten Hecken die Nutzung dieses Lebensraums als fakultativen Brutort für die Mehrzahl der Arten in vielen der untersuchten Hecken.

Etwa ein Viertel der Arten besiedelte die Hälfte der untersuchten Hecken alljährlich (Tab. 22). Diese Arten gehörten zu den häufigsten Heckenbewohnern. Eine Ausnahme bildete nur der Zaunkönig, der ausschließlich in Hecke 97 in allen Untersuchungsjahren zwei bis drei Reviere hatte. Die Goldammer wurde in allen Hecken festgestellt und fehlte nur in einem Jahr in der bereits erwähnten Waldrandhecke 51 .

Mönchs- und Dorngrasmücke, Amsel, Heckenbraunelle und Feldsperling fehlten in weniger als einem Drittel der genutzten Hecken zeitweilig, während Zilpzalp, Singdrossel, Kohlmeise und Gartengrasmücke in 50-60 \% aller von ihnen genutzten Hecken jedes Jahr siedelten.

Der Anteil der Hecken, die von den jeweiligen Arten in allen drei Jahren genutzt worden sind, betrug $49 \%$ gegenüber den verbleibenden Hecken, die nur in einem oder zwei Jahren Reviere der betreffenden Art aufwiesen.

Der Anteil der Reviere, die in Hecken lagen, die nicht über alle Untersuchungsjahre von der betreffenden Art besiedelt worden sind, lag nur bei fünf Arten unter $10 \%$ : Goldammer, Heckenbraunelle, Amsel, Feldsperling und Mönchsgrasmücke (Tab. 22).

$24,2 \%$ aller Reviere wurden in Hecken festgestellt, in denen die jeweilige Art nicht alljährlich brütete. Unter diesem Wert lagen, neben den fünf oben genannten Arten, mit Dorngrasmücke, Singdrossel, Zilpzalp und Gartengrasmücke nur noch vier weitere Arten. Die übrigen 32 Arten hatten überdurchschnittlich viele Reviere in Hecken, die sie nur in ein oder zwei Jahren besetzten.

\subsection{Kumulative Artzusammensetzung}

Die Anordnung der Hecken in Tab. 20 folgt einer sukzessiven Zunahme der Artenzahl von oben nach unten. Die geringste Anzahl von Arten lag bei vier (über die Untersuchungsjahre summiert, in vier Hecken festgestellt), die höchste bei 31 in einer Hecke. In Tab. 23 wurden Arten, die sich mit einer bestimmten Anzahl von weiteren Arten in den Hecken einstellen, aufgrund ihres sukzessiven Erscheinens in Gruppen zusammengefasst. 
Tab. 23: Arten, die in den untersuchten 30 Hecken (zusätzlich) auftreten, in denen die jeweils angegebene Artenzahl vorhanden ist, eingeklammert ist die Anzahl der Hecken mit der entsprechenden Artenzahl.

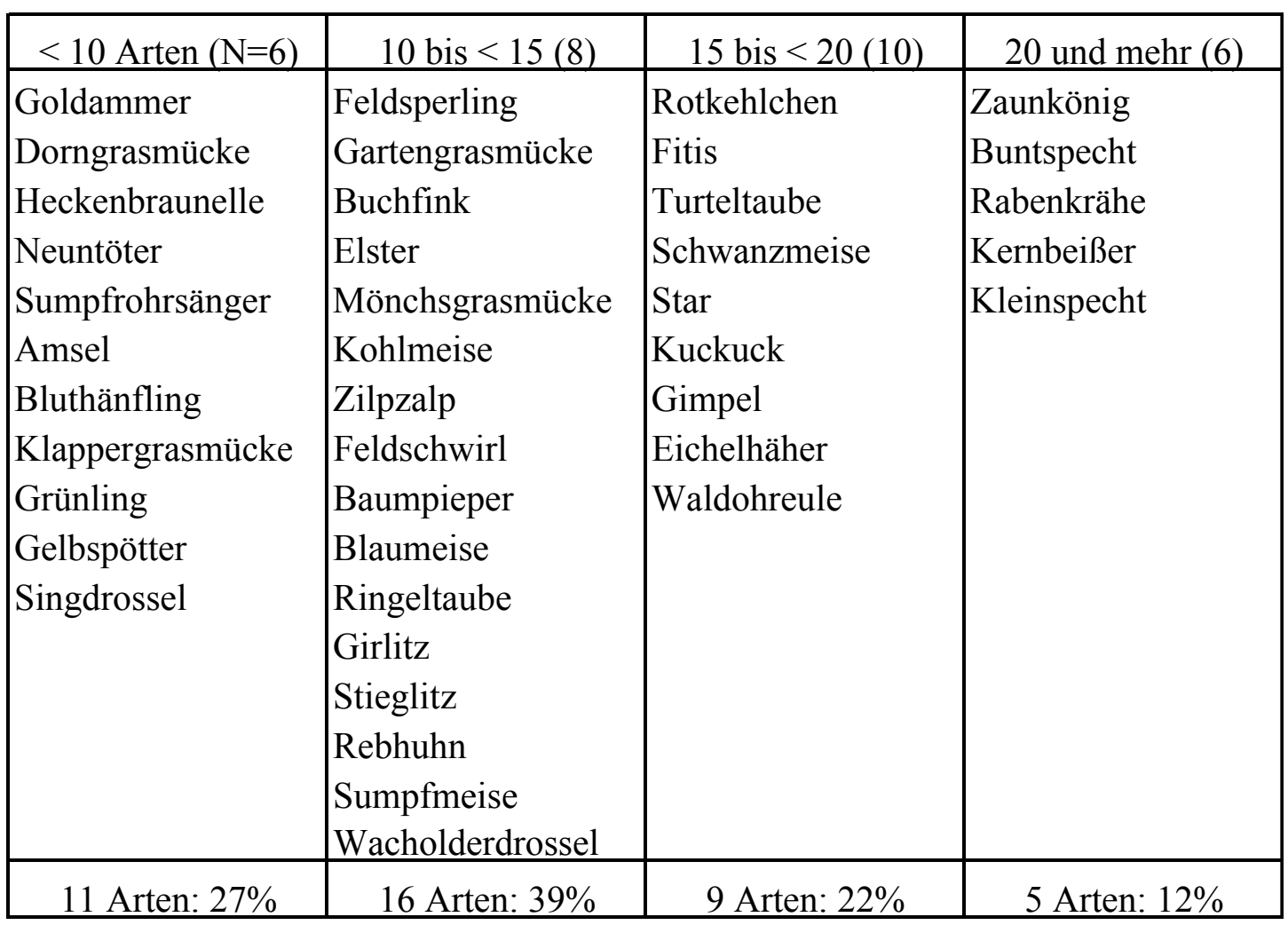

Aus Tab. 23 geht hervor, dass bereits in Hecken, die jeweils weniger als 15 Arten als Brutvögel beherbergen, 65 \% aller nachgewiesenen Arten vorkommen können. Bereits in den vier artenärmsten Hecken, die jede für sich nur vier Arten aufwies, kamen mit Goldammer, Dorngrasmücke, Sumpfrohrsänger, Neuntöter, Heckenbraunelle, Amsel und Klappergrasmücke sieben Brutvogelarten vor (Tab. 20). In Hecken, die mehr als 20 Arten aufwiesen, traten nur fünf weitere Arten auf.

\subsection{Der Einfluss von Heckenstrukturen und Landschaftselementen auf die Abundanz von Brutvögeln in Hecken}

Unter Einbeziehung aller Arten, die im Bereich der untersuchten Hecken vorkamen (mit Ausnahme der Feldlerche) wurden 44 Arten nachgewiesen, die jeweils in mindestens einem Jahr ein Revier in oder an einer Hecke besetzt hatten (Tab. 24). Mit Wachtel, Wiesenpieper und Bachstelze kamen drei Arten hinzu, die vorher nicht als Heckenvögel berücksichtigt wurden, weil sie weder durch ihre Neststandorte noch durch Habitatbindung bezüglich ihrer Nahrungssuche unmittelbar an Hecken gebunden sind. In den folgenden Analysen sollten aber auch Einflüsse der Umgebung von Hecken mit berücksichtigt werden und deshalb wurde das Spektrum der betrachteten Arten erweitert. 
Tab. 24: Abundanz [BP/km] und Dominanz [\%] der Arten in 30 Hecken zwischen 1998-2000. Gekennzeichnete Arten brüteten *teilweise oder **ausschließlich in Nistkästen.

\begin{tabular}{|c|c|c|c|c|c|c|c|}
\hline & Art & $\mathrm{BP} / \mathrm{Km}$ & Dominanz & & Art & $\mathrm{BP} / \mathrm{Km}$ & Dominanz \\
\hline 1 & Goldammer & 11,52 & 12,87 & 23 & Schwanzmeise & 0,86 & 0,96 \\
\hline 2 & Heckenbraunelle & 7,64 & 8,54 & 24 & Stieglitz & 0,80 & 0,89 \\
\hline 3 & Amsel & 7,19 & 8,03 & 25 & Feldschwirl & 0,74 & 0,83 \\
\hline 4 & Dorngrasmücke & 6,90 & 7,71 & 26 & Baumpieper & 0,68 & 0,76 \\
\hline 5 & Feldsperling** & 6,27 & 7,01 & 27 & Gimpel & 0,57 & 0,64 \\
\hline 6 & Mönchsgrasmücke & 5,13 & 5,74 & 28 & Turteltaube & 0,51 & 0,57 \\
\hline 7 & Gartengrasmücke & 4,73 & 5,29 & 29 & Kuckuck & 0,46 & 0,51 \\
\hline 8 & Singdrossel & 4,16 & 4,65 & 30 & Zaunkönig & 0,46 & 0,51 \\
\hline 9 & Klappergrasmücke & 3,48 & 3,89 & 31 & Buntspecht & 0,34 & 0,38 \\
\hline 10 & Zilpzalp & 3,42 & 3,82 & 32 & Wiesenpieper & 0,34 & 0,38 \\
\hline 11 & Kohlmeise* & 3,14 & 3,51 & 33 & Sumpfmeise & 0,34 & 0,38 \\
\hline 12 & Grünling & 2,57 & 2,87 & 34 & Eichelhäher & 0,34 & 0,38 \\
\hline 13 & Buchfink & 2,51 & 2,80 & 35 & Gelbspötter & 0,29 & 0,32 \\
\hline 14 & Sumpfrohrsänger & 2,11 & 2,36 & 36 & Rebhuhn & 0,29 & 0,32 \\
\hline 15 & Blaumeise* & 1,65 & 1,85 & 37 & Bachstelze & 0,23 & 0,25 \\
\hline 16 & Bluthänfling & 1,60 & 1,78 & 38 & Wacholderdrossel & 0,23 & 0,25 \\
\hline 17 & Rotkehlchen & 1,43 & 1,59 & 39 & Star & 0,23 & 0,25 \\
\hline 18 & Ringeltaube & 1,37 & 1,53 & 40 & Waldohreule & 0,17 & 0,19 \\
\hline 19 & Elster & 1,25 & 1,40 & 41 & Rabenkrähe & 0,17 & 0,19 \\
\hline 20 & Fitis & 1,08 & 1,21 & 42 & Kernbeißer & 0,11 & 0,13 \\
\hline 21 & Neuntöter & 1,08 & 1,21 & 43 & Kleinspecht & 0,06 & 0,06 \\
\hline 22 & Wachtel & 0,97 & 1,08 & 44 & Girlitz & 0,06 & 0,06 \\
\hline
\end{tabular}

Die Goldammer ist der häufigste Brutvogel in den Untersuchungsgebieten. Weitere dominante Arten (>5\%) sind Heckenbraunelle, Amsel, Dorngrasmücke, Feldsperling sowie Mönchs- und Gartengrasmücke. Die häufigsten 22 Arten bilden über $90 \%$ aller Reviere, die in den Hecken festgestellt wurden. Die Shannon-Wiener-Diversität beträgt über alle Hecken $\mathrm{H}_{\mathrm{s}}=3,1$.

\subsubsection{Ergebnis der Multiplen Linearen Regression}

Mit einer Multiplen Regression wurden die wichtigsten Heckenparameter zur Beschreibung der Brutpaardichte in den Hecken analysiert (Tab. 25). Das Modell ist signifikant mit $p=0,038$ für N=30 Hecken mit 69 \% erklärter Varianz, bei fünf berücksichtigten Variablen.

Tab. 25: Multiple Lineare Regression für Brutpaare je Kilometer Hecke mit korr. $R^{2}=0,69, F$ $(5,24)=13,923, p<0,001$, SF der Schätzung: 23,078, $N=30$.

\begin{tabular}{|l|cccccc|}
\hline & Beta & Stdf. Beta & B & Stdf. B & t (24) & $p$ \\
\hline Konst. & & & 35,468 & 16,181 & 2,192 & 0,038 \\
Breite & 0,605 & 0,169 & 16,157 & 4,508 & 3,584 & 0,001 \\
Länge & $-0,283$ & 0,107 & $-0,102$ & 0,038 & $-2,639$ & 0,014 \\
Wegedichte & $-0,243$ & 0,113 & $-0,044$ & 0,020 & $-2,159$ & 0,041 \\
Heckenabundanz & 0,137 & 0,114 & 0,026 & 0,021 & 1,195 & 0,244 \\
Überhälter & 0,155 & 0,149 & 4,857 & 4,661 & 1,042 & 0,308 \\
\hline
\end{tabular}


Die wichtigsten Heckenparameter die die Gesamtabundanz der Brutvögel bestimmen, sind Breite, Länge und Wegedichte. Die Stärke des Einflusses der Variablen 'Breite' $(B e t a=0,6)$ ist mehr als doppelt so stark wie der der folgenden Variablen 'Länge' (Beta=-0,28). Je breiter Hecken angelegt sind, desto höher ist die Brutvogeldichte (Abb. 24, A). Kurze Hecken und Hecken, in deren näherer Umgebung das Wegenetz nicht allzu dicht ist, sowie die Anzahl der Hecken in der Umgebung (Heckenabundanz) und die Höhe der Hecke (Anzahl der Überhälter) begünstigen die Abundanz der Brutvögel (Abb. 24, B).
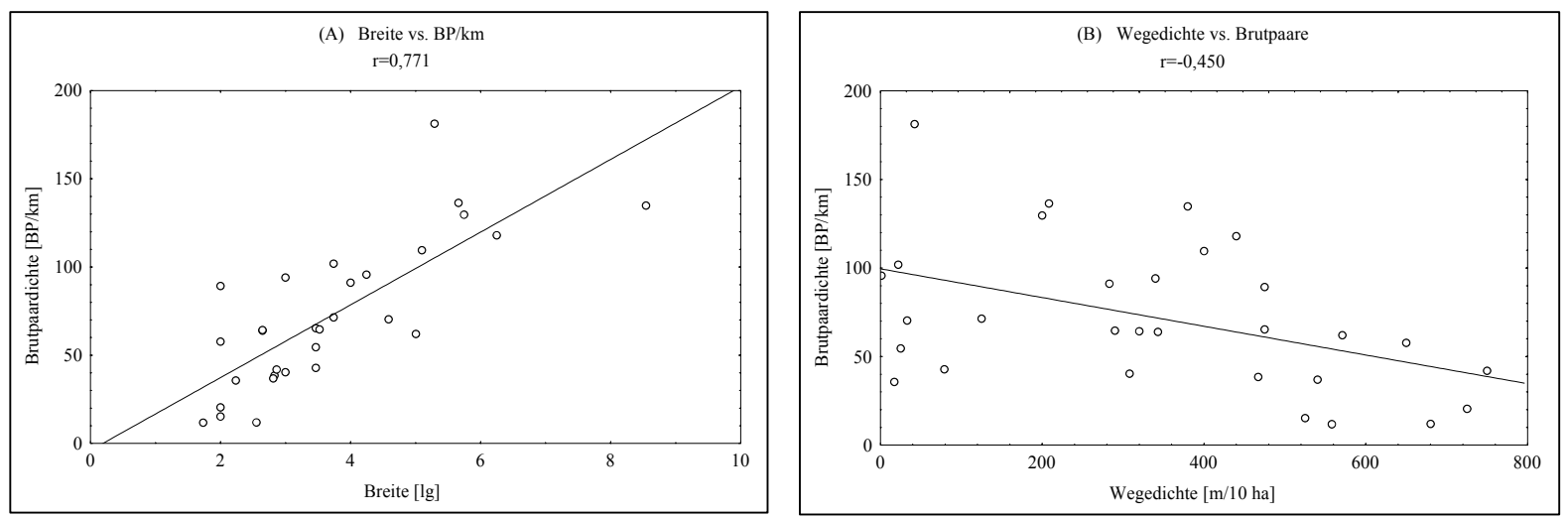

Abb. 24: Beziehung zwischen Brutpaaren pro Kilometer und Heckenbreite (A) und Wegedichte (B).

Während die Artenzahl mit zunehmender Heckenlänge ansteigt, ist die Revierdichte in kürzeren Hecken höher als in langen Hecken (Abb. 25).
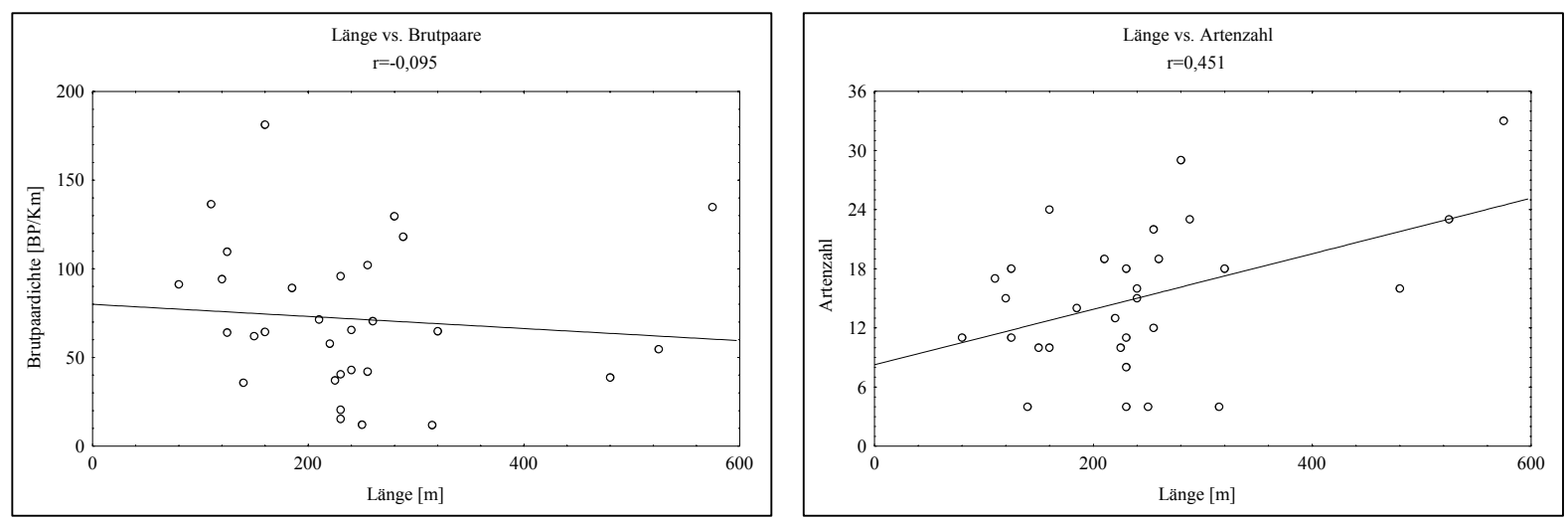

Abb. 25: Beziehung Brutpaare pro Kilometer bzw. Artenzahl mit Länge der Hecken.

Anhand einer Hauptkomponentenanalyse (Tab. 26) zeigt sich die Bedeutung von den Variablen 'Breite' und 'Überhälter'. Beide Variablen beschreiben das Gehölzvolumen der Hecke und wirken in die gleiche Richtung. Beachtenswert sind auch die Ladungen der Variablen 'Länge' und 'Heckendichte', die beide nur knapp unter einem Wert von 0,6 lagen und ökologisch ebenfalls das Gehölzvolumen beschreiben. 'Brache' und 'Acker' sowie 'Waldnähe' und dichter 'Heckenverbund' bei gleichzeitig großer Entfernung zur nächsten Siedlung ('Siedlungsnähe') sind die bestimmenden Variablen in den Faktoren 2 und 3, für die aber kein Einfluss auf die 
Brutpaardichte in Hecken gezeigt werden konnte. Heckendichte und Wegedichte sind dagegen als Variablen im Modell enthalten.

Aus der PCA ergeben sich fünf signifikante Hauptkomponenten (Eigenwerte dieser Faktoren sind größer 1, Kaiser-Guttman Kriterium, nach BORTZ 1995) mit zusammen 80 \% erklärter Varianz. Nach BORTZ (1995) sind für die Faktoren nur Polaritäten mit Ladungen über 0,6 bedeutsam, diese sind in Tab. 26 gekennzeichnet.

Tab. 26: Ergebnis der Hauptkomponentenanalyse: Faktorladungen (Varimax standard Rotation) und Extraktion der Hauptkomponenten mit Ladungen >0,6 markiert.

\begin{tabular}{|l|rrrrr|}
\hline & \multicolumn{5}{|c}{ Faktor } \\
\cline { 2 - 5 } & 1 & 2 & 3 & 4 & 5 \\
\hline Breite & 0,889 & $-0,039$ & 0,091 & 0,298 & 0,114 \\
Überhälter & 0,806 & 0,120 & 0,130 & 0,262 & 0,001 \\
Brache & $-0,114$ & $-0,966$ & 0,000 & 0,091 & 0,018 \\
Acker & $-0,055$ & 0,960 & 0,044 & $-0,046$ & 0,027 \\
Siedlungsnähe & $-0,104$ & 0,306 & 0,846 & 0,132 & 0,254 \\
Heckenverbund & $-0,314$ & 0,251 & $-0,729$ & 0,155 & 0,342 \\
Waldnähe & $-0,272$ & 0,061 & $-0,626$ & 0,512 & 0,212 \\
Dichtewert & 0,187 & $-0,010$ & 0,050 & 0,884 & $-0,028$ \\
Wegedichte & $-0,047$ & 0,094 & 0,120 & $-0,764$ & 0,088 \\
Krautsaum & 0,281 & $-0,036$ & $-0,066$ & $-0,162$ & 0,889 \\
Heckendichte & 0,538 & $-0,479$ & $-0,063$ & $-0,181$ & $-0,477$ \\
Länge & 0,561 & $-0,015$ & 0,041 & $-0,287$ & 0,176 \\
\hline Erk1.Varianz & 2,359 & 2,272 & 1,693 & 1,978 & 1,297 \\
Gesamtvarianz [\%] & 19,7 & 18,9 & 14,1 & 16,5 & 10,8 \\
Summe Varianz [\%] & 19,7 & 38,6 & 52,7 & 69,2 & 80,0 \\
\hline
\end{tabular}

\subsection{Einfluss von Heckenstrukturen auf die Dichte einzelner Vogelarten}

Im Folgenden sollen die Zusammenhänge zwischen 12 Variablen, die die Hecken beschreiben, und die Häufigkeit einzelner Vogelarten mit einer Kanonischen Korrelation analysiert werden. Die Methode erlaubt die Auswahl der 14 häufigsten Arten, wobei Feldsperling, Kohlund Blaumeise ausgeschlossen wurden, weil die Abundanz dieser Arten stärker vom Angebot künstlicher Nistkästen als von den Heckenstrukturen abhängt.

Der $\mathrm{Chi}^{2}$-Vortest zur Feststellung der Anzahl extrahierbarer Roots lässt lediglich die Betrachtung eines Beziehungspaares zu (bei Entfernung eines weiteren Roots wird p>0,05, Tab. 27).

Tab. 27: Chi²-Test zur Feststellung der Anzahl extrahierbarer Roots.

\begin{tabular}{|c|cccccc|}
\hline & Kanon. $\mathrm{R}$ & Kanon. $\mathrm{R}^{2}$ & $\mathrm{Chi}^{2}$ & $\mathrm{FG}$ & $\mathrm{p}$ & Lbda. Prime \\
\hline 0 & 0,992 & 0,984 & 228 & 168 & 0,002 & 0,001 \\
1 & 0,970 & 0,941 & 164 & 143 & 0,113 & 0,001 \\
2 & 0,940 & 0,883 & 120 & 120 & 0,486 & 0,001 \\
3 & 0,882 & 0,778 & 87 & 99 & 0,809 & 0,004 \\
\hline
\end{tabular}


In Tab. 28 ist das Ergebnis der Kanonischen Korrelation dargestellt. 'Breite' und 'Überhälter' zeigen innerhalb der Faktorenstruktur für die Heckenvariablen die höchsten Werte. Diese sind als Prädiktorvariablen für die Häufigkeit von Amsel, Zilpzalp, Mönchs- und Gartengrasmücke, sowie Singdrossel, Klappergrasmücke, Buchfink und Heckenbraunelle (Kriteriumsvariablen) am besten geeignet.

Tab. 28: Ergebnis der Kanonischen Korrelation: Faktorenstruktur und Extraktion markierter Ladungen.

\begin{tabular}{|ll|ll|}
\hline \multicolumn{2}{|c|}{ Heckenvariablen } & \multicolumn{2}{c|}{ Arten } \\
\hline Breite & 0,778 & Amsel & 0,874 \\
Überhälter & 0,760 & Zilpzalp & 0,869 \\
Heckendichte & 0,479 & Mönchsgrasmücke & 0,850 \\
Heckenabundanz & 0,439 & Gartengrasmücke & 0,785 \\
Siedlung & 0,052 & Singdrossel & 0,668 \\
Acker & 0,039 & Klappergrasmücke & 0,650 \\
Länge & 0,017 & Buchfink & 0,641 \\
Brache & 0,010 & Heckenbraunelle & 0,628 \\
Waldnähe & $-0,038$ & Rotkehlchen & 0,421 \\
Krautsaum & $-0,101$ & Goldammer & 0,401 \\
Wegedichte & $-0,267$ & Grünling & 0,200 \\
Heckenverbund & $-0,436$ & Sumpfrohrsänger & 0,079 \\
& & Dorngrasmücke & $-0,150$ \\
& & Bluthänfling & $-0,186$ \\
\hline
\end{tabular}

Die 'Volumen-Variablen' Heckenbreite und -höhe bieten keine Vorhersagemöglichkeit für die Häufigkeit von Bluthänfling, Dorngrasmücke, Sumpfrohrsänger, Grünling, Goldammer und Rotkehlchen.

\subsection{Bruterfolg}

Von 14 offen brütenden Singvogelarten wurden 239 Nester mit mindestens einem Ei oder Jungvogel gefunden und kontrolliert: Amsel (36), Buchfink (4), Dorngrasmücke (34), Gartengrasmücke (21), Goldammer (45), Grünling (2), Hänfling (7), Heckenbraunelle (19), Klappergrasmücke (7), Mönchsgrasmücke (22), Neuntöter (3), Singdrossel (24), Sumpfrohrsänger (5) und Zilpzalp (10).

In Tab. 29 ist der Nesterfolg und der Anteil ausgeraubter bzw. anderweitig verlorener Nester über alle Arten nach den Jahren differenziert zusammengestellt. Von allen 239 gefundenen Nestern waren weniger als ein Drittel erfolgreich. Die Hauptursache für Nestverluste war Prädation, $54 \%$ aller kontrollierten Nester wurden ausgeraubt. Weitere Verlustursachen beruhen auf Witterungseinflüssen, Störungen, Mahd und der Aufgabe von Gelegen aus ungeklärter Ursache. 
Tab. 29: Anzahl und Anteil der erfolgreichen und der durch Prädation oder andere Ursachen verlorenen Nester.

\begin{tabular}{|c|c|cc|cc|cc|}
\hline & N & erfolgreich & $\%$ & Prädation & $\%$ & sonstige Verluste & $\%$ \\
\hline 1998 & 39 & 11 & 28,2 & 21 & 53,8 & 7 & 17,9 \\
1999 & 92 & 27 & 29,3 & 47 & 51,1 & 18 & 19,6 \\
2000 & 108 & 35 & 32,4 & 61 & 56,5 & 12 & 11,1 \\
\hline gesamt & 239 & 73 & 30,5 & 129 & 54,0 & 37 & 15,5 \\
\hline
\end{tabular}

Zwischen 1998 und 1999 nahm der Anteil erfolgreicher Nester um einen Prozentwert zu und der Anteil ausgeraubter Nester ging um 2,7 zurück. Von 1999 auf 2000 nahmen sowohl die Prädationsrate als auch der Nesterfolg zu. Die Zunahme erfolgreicher Nester beträgt $9 \%$, die Prädation stieg um 10,5\% an.

Der Bruterfolg einzelner Arten ist in einer detaillierten Aufgliederung der einzelnen brutbiologischen Kennwerte in Tab. 30 zusammengestellt.

Tab. 30: Bruterfolg häufiger Brutvögel in Hecken, 1998-2000.

\begin{tabular}{|c|c|c|c|c|c|c|c|c|c|c|c|c|c|}
\hline & 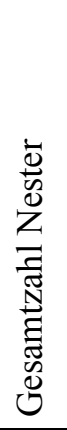 & 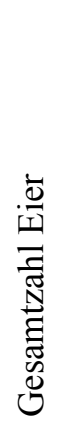 & 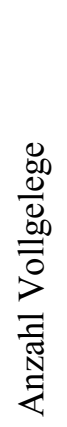 & 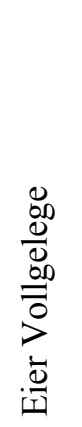 & 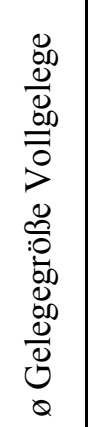 & 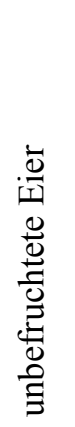 & 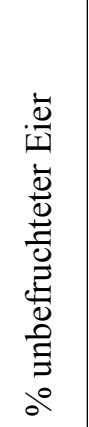 & 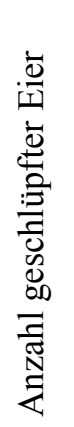 & 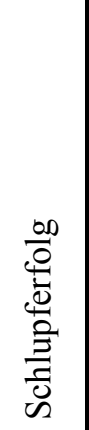 & 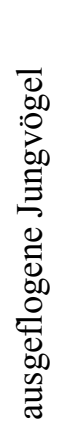 & 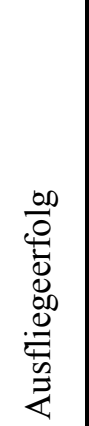 & 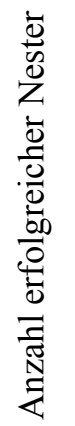 & $\begin{array}{l}\frac{b 0}{0} \\
\stackrel{0}{0} \\
\frac{\mathbb{E}}{0} \\
Z\end{array}$ \\
\hline Amsel & 36 & 140 & 27 & 112 & 4,15 & 2 & 4,7 & 41 & 36,6 & 20 & 0,56 & 5 & 0,14 \\
\hline Singdrossel & 24 & 84 & 17 & 70 & 4,12 & 2 & 7,1 & 26 & 37,1 & 14 & 0,58 & 4 & 0,17 \\
\hline Goldammer & 45 & 176 & 33 & 139 & 4,21 & 20 & 18,9 & 86 & 61,9 & 56 & 1,24 & 19 & 0,42 \\
\hline Dorngrasmücke & 34 & 137 & 27 & 120 & 4,44 & 3 & 3,4 & 86 & 71,1 & 76 & 2,24 & 17 & 0,5 \\
\hline Mönchsgrasmücke & 22 & 102 & 18 & 88 & 4,89 & 3 & 5,0 & 57 & 64,8 & 37 & 1,68 & 8 & 0,36 \\
\hline Gartengrasmücke & 21 & 76 & 13 & 61 & 4,69 & 1 & 2,1 & 46 & 75,4 & 32 & 1,52 & 7 & 0,33 \\
\hline Klappergrasmücke & 7 & 30 & 6 & 29 & 4,83 & 2 & 14,3 & 12 & 41,4 & 7 & 1 & 2 & 0,29 \\
\hline Heckenbraunelle & 19 & 74 & 12 & 65 & 5,42 & 6 & 27,3 & 16 & 26,6 & 15 & 0,79 & 4 & 0,21 \\
\hline Zilpzalp & 10 & 54 & 10 & 54 & 5,4 & 3 & 7,7 & 36 & 66,7 & 26 & 2,6 & 7 & 0,7 \\
\hline
\end{tabular}

Die Gelegegröße liegt bei Amsel, Singdrossel und Goldammer bei etwa 4,2 Eiern je Vollgelege. Bei den Grasmücken umfasst ein Gelege 4,65 (+/- 0,25) Eier. Die höchsten Werte unter den untersuchten Arten hatten Heckenbraunelle und Zilpzalp mit einer durchschnittlichen Gelegegröße von 5,4. 
Der Schlupferfolg war bei der Heckenbraunelle mit nur etwa einem Viertel erfolgreich geschlüpfter Eier am geringsten. Amsel, Singdrossel und Klappergrasmücke lagen um $40 \%$, die übrigen Arten hatten einen Schlupferfolg zwischen 60-75 \%.

Der Ausfliegeerfolg bei Amsel, Singdrossel und Heckenbraunelle lag bei weniger als einem Jungvogel je angefangener Brut. Bei der Dorngrasmücke wurden jeweils mehr als zwei Jungvögel je Nest flügge.

Bei Amsel, Singdrossel und Heckenbraunelle war nur jedes 5. Nest erfolgreich, bei der Goldammer lag der Nesterfolg doppelt so hoch. Die Dorngrasmücke war mit $50 \%$ deutlich erfolgreicher als die übrigen Grasmückenarten, deren Nesterfolg zwischen 29-36 \% lag. Mit 70 \% hatte der Zilpzalp den höchsten Nesterfolg.

Tab. 31: Ermittlung der durchschnittlichen Nestdauer (ND) aus beobachteter Brutdauer und der mittleren Nestlingszeit nach Literaturangaben.

\begin{tabular}{|c|c|c|c|c|c|c|c|}
\hline & 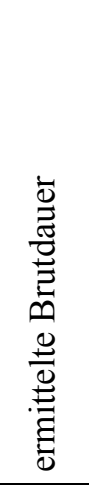 & 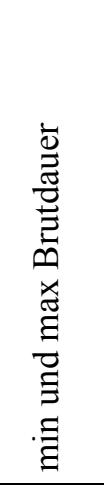 & 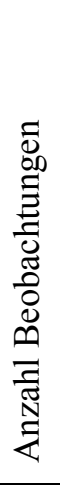 & 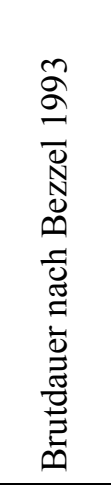 & 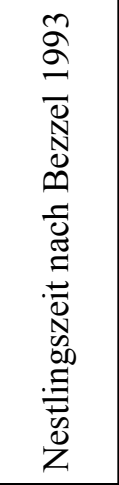 & 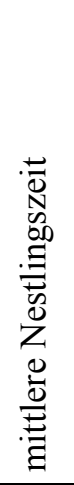 & 宗 \\
\hline Amsel & 14 & $12-15$ & 5 & $11-16$ & $12-19$ & 15 & 29 \\
\hline Singdrossel & 13,7 & $11-15$ & 3 & $10-17$ & $13-14$ & 13 & 26,7 \\
\hline Goldammer & 12,8 & $12-13$ & 5 & $11-14$ & $9-14$ & 11 & 23,8 \\
\hline Dorngrasmücke & 11,6 & $10-13$ & 8 & $10-14$ & $10-14$ & 12 & 23,6 \\
\hline Mönchsgrasmücke & 12 & 12 & 1 & $10-16$ & $10-15$ & 12 & 24 \\
\hline Gartengrasmücke & 13,6 & $13-14$ & 3 & $11-15$ & $9-14$ & 11 & 24,6 \\
\hline Klappergrasmücke & (13) & $(-)$ & 0 & $11-15$ & $11-13$ & 12 & 25 \\
\hline Heckenbraunelle & 12 & 12 & 1 & $11-13$ & $11-15$ & 13 & 25 \\
\hline Zilpzalp & 14 & 14 & 1 & $13-15$ & $14-15$ & 14 & 28 \\
\hline
\end{tabular}

Für die Klappergrasmücke musste zur Angabe der Brutdauer auf Literaturangaben zurückgegriffen werden. Auch bei Arten, für die nur wenige Beobachtungen zur Bebrütungszeit vorlagen (Mönchsgrasmücke, Heckenbraunelle und Zilpzalp), wurde zur Ermittlung der Nestlingszeit die jeweils im Untersuchungsgebiet beobachtete Brutdauer verwendet. 
Tab. 32: Die Überlebenswahrscheinlichkeit $\left(\operatorname{SURV}_{(\mathrm{t})}\right)$ von Singvogelnestern in Hecken berechnet nach der Mayfield-Methode. Zu den verwendeten Abkürzungen siehe Text und Methodik.

\begin{tabular}{|l|ccccc|c|}
\hline & N & EXP & L & MORT(d) & SURV(d) & SURV(t) \\
\hline Amsel & 36 & 453 & 31 & 0,0684 & 0,9316 & 12,8 \\
Singdrossel & 24 & 280,5 & 20 & 0,0713 & 0,9287 & 13,7 \\
Goldammer & 45 & 570,5 & 26 & 0,0456 & 0,9544 & 33,0 \\
Dorngrasmücke & 34 & 455 & 17 & 0,0374 & 0,9626 & 40,7 \\
Mönchsgrasmücke & 22 & 269,5 & 14 & 0,0519 & 0,9481 & 27,8 \\
Gartengrasmücke & 21 & 310 & 14 & 0,0452 & 0,9548 & 32,1 \\
Klappergrasmücke & 7 & 92,5 & 5 & 0,0540 & 0,9460 & 24,9 \\
Heckenbraunelle & 19 & 202 & 15 & 0,0743 & 0,9257 & 14,5 \\
Zilpzalp & 10 & 180,5 & 2 & 0,0166 & 0,9834 & 62,6 \\
\hline
\end{tabular}

Die höchsten täglichen Mortalitätswahrscheinlichkeiten $\left(\mathrm{MORT}_{(\mathrm{d})}\right)$ von etwa 0,07 traten bei Heckenbraunelle, Singdrossel und Amsel auf (Tab. 32). Mönchs- und Klappergrasmücke hatten eine tägliche Verlustwahrscheinlichkeit von über 0,05, Goldammer und Gartengrasmücke von etwas darüber. Die Nester von Dorngrasmücke $(0,96)$ und Zilpzalp $(0,98)$ hatten die höchste Überlebenswahrscheinlichkeit $\left(\operatorname{SURV}_{(\mathrm{d})}\right)$, so dass die Bruten dieser Arten mit über 0,4 bzw. 0,6 die höchste Gesamt-Überlebenswahrscheinlichkeit $\left(\mathrm{SURV}_{(\mathrm{t})}\right)$ hatten. Bei der Amsel war dieser Wert am geringsten: Nur 12,8 \% aller Nester überlebten. Auch bei Singdrossel und Heckenbraunelle hatten weniger als 15 \% aller Gelege Erfolg. Bei Mönchs- und Klappergrasmücke überlebte etwa ein Viertel der Gelege, bei Gartengrasmücke und Goldammer ein Drittel.

Tab. 33: Ausfliegeerfolg berechnet aus der mittleren Gelegegröße (ø Eizahl, Tab. 30) aller Vollgelege und der Gesamt-Überlebenswahrscheinlichkeit von Nestern (SURV ${ }_{t}$ aus Tab. 32). Zum Vergleich die aus der Anzahl der ausgeflogenen Jungvögel je Nest ermittelten Werte aus Tab. 30.

\begin{tabular}{|l|cc|}
\hline & \multicolumn{2}{|c|}{ Ausfliegeerfolg } \\
& $\varnothing$ Eizahl * SURV(t) & Jungvögel je Nest \\
\hline Amsel & 0,53 & 0,56 \\
Singdrossel & 0,56 & 0,58 \\
Goldammer & 1,39 & 1,24 \\
Dorngrasmücke & 1,81 & 2,24 \\
Mönchsgrasmücke & 1,36 & 1,68 \\
Gartengrasmücke & 1,51 & 1,52 \\
Klappergrasmücke & 1,20 & 1,00 \\
Heckenbraunelle & 0,81 & 0,79 \\
Zilpzalp & 3,38 & 2,60 \\
\hline
\end{tabular}

Mit den nach der Mayfield-Methode berechneten Überlebenswahrscheinlichkeiten von Singvogelnestern lassen sich die durchschnittlich überlebenden Jungvögel aus der mittleren Gelegegröße der Population ermitteln. Damit ergibt sich ein zweiter Weg zur Beschreibung 
des Ausfliegeerfolgs anhand im Untersuchungsgebiet ermittelter Überlebensraten und Gelegegrößen (Tab. 33).

Die anhand der Überlebenswahrscheinlichkeit von Nestern berechneten Ausfliegeerfolge von Dorn- und Mönchsgrasmücke liegen deutlich unter den Werten, die als Anzahl aller ausgeflogenen Jungvögel über die Summe aller Nester ermittelt wurden (-21\%). Bei Goldammer $(+12 \%)$, Klappergrasmücke (+20\%) und vor allem Zilpzalp (+30\%) ist es umgekehrt. Der Ausfliegeerfolg von Amsel, Singdrossel, Gartengrasmücke und Heckenbraunelle erreicht durch beide Berechnungsmethoden nahezu identische Werte.

\subsection{Populationsentwicklung}

Die Erfassung des Ausfliegeerfolgs (Anzahl flügger Jungvögel je Nest) ermöglicht eine Abschätzung der Populationsgröße für das darauffolgende Jahr, wenn Daten zur jährlichen Sterblichkeitsrate der betreffenden Arten vorliegen. Für die in dieser Studie durchgeführten Berechnungen wurden dazu Daten aus der Literatur (Tab. 34) verwendet.

Um vom Ausfliegeerfolg auf den Reproduktionserfolg eines Brutpaares als Anzahl der flüggen Jungvögel über die gesamte Brutdauer schließen zu können, wurde die Anzahl der Brutversuche der Arten mit in die Berechnung einbezogen. Ich habe dabei zwischen Arten mit einer oder zwei Jahresbruten unterschieden und mit der Addition von 0,5 versucht, potentielle Nachgelege zu berücksichtigen. Die in Tab. 34 unter $\mathrm{B}_{(\mathrm{S})}$ angegebenen Werte basieren auf Daten aus dem Common Bird Census (CBC) in UK und sind einer brutökologischen Charakterisierung von Feldvögeln nach SIRIWARDENA et al. (1998) entnommen.

Tab. 34: Mortalitätsraten für Alt- $\left(M_{a d}\right)$ und Jungvögel $\left(M_{j u v}\right)$ nach Literaturangaben und Anzahl der Brutversuche $B$ und $B_{S}$ nach Angaben von SIRIWARDENA et al. (1998), Schätzwerte kursiv.

\begin{tabular}{|c|c|c|c|c|c|}
\hline & \multicolumn{3}{|c|}{ Mortalität } & \multicolumn{2}{|c|}{ Brutversuche } \\
\hline & $\mathrm{M}(\mathrm{ad})$ & $M(j u v)$ & Quelle & $\mathrm{B}$ & $\mathrm{B}(\mathrm{S})$ \\
\hline Amsel & 0,45 & 0,69 & Glutz von Blotzheim \& Bauer1988 & 2,5 & $(2,8)$ \\
\hline Singdrossel & 0,44 & 0,58 & Glutz von Blotzheim \& Bauer1988 & 2,5 & $(2,5)$ \\
\hline Goldammer & 0,46 & 0,52 & Glutz von Blotzheim \& Bauer1997 & 2,5 & $(2,0)$ \\
\hline Dorngrasmücke & 0,62 & 0,57 & Glutz von Blotzheim \& Bauer 1991 & 1,5 & $(1,3)$ \\
\hline Mönchsgrasmücke & 0,61 & 0,68 & Kohler 1975, Bairlein 1978, zit. in Glutz \& Bauer1991 & 1,5 & $(1,5)$ \\
\hline Gartengrasmücke & 0,52 & 0,70 & Kohler 1975, zit. in Glutz \& Bauer1991 & 1,5 & $(1,0)$ \\
\hline Klappergrasmücke & 0,62 & 0,47 & Kohler 1975, zit. Glutz von Blotzheim \& Bauer1991 & 1,5 & $(1,0)$ \\
\hline Heckenbraunelle & 0,32 & 0,5 & Snow 1982 für GB, zit. in Glutz \& Bauer1985 & 2,5 & $(2,0)$ \\
\hline Zilpzalp & 0,70 & 0,6 & Glutz von Blotzheim \& Bauer1991 & 2,5 & $(2,0)$ \\
\hline
\end{tabular}

Die unter Annahme von Ortstreue und auf eigenem Recruitment basierende relative Populationsgröße (ausgehend von 100 Brutpaaren) für ein Folgejahr ist in Tab. 35 für neun 
Singvogelarten in Hecken dargestellt. Unter $\mathrm{P}_{(\mathrm{t}+1)}$ mit $\mathrm{B}_{(\mathrm{S})}$ ist in der zweiten Spalte die Berechnung auf der Basis der von SIRIWARDENA et al. (1998) angegebenen Brutversuche durchgeführt. Unter $\mathrm{P}_{(\mathrm{t}+1)[\text { Mayfield] }}$ ist die relative Populationsgröße im Folgejahr mit nach der Mayfield-Methode berechnetem Ausfliegeerfolg angegeben.

Tab. 35: Relative Populationsgröße [BP] heckenbrütender Singvögel in einem Folgejahr, basierend auf den erhobenen Ausfliegeerfolgen der Arten. Zu den unterschiedlichen Berechnungsvarianten siehe Text.

\begin{tabular}{|l|ccc|}
\hline & $\mathrm{P}(\mathrm{t}+1)$ & $\mathrm{P}(\mathrm{t}+1)$ mit $\mathrm{B}(\mathrm{S})$ & $\mathrm{P}(\mathrm{t}+1)$ [Mayfield] \\
\hline Amsel & 77 & 79 & 76 \\
Singdrossel & 87 & 87 & 85 \\
Goldammer & 128 & 114 & 137 \\
Dorngrasmücke & 110 & 101 & 96 \\
Mönchsgrasmücke & 79 & 79 & 72 \\
Gartengrasmücke & 82 & 71 & 82 \\
Klappergrasmücke & 78 & 65 & 86 \\
Heckenbraunelle & 121 & 112 & 123 \\
Zilpzalp & 160 & 134 & 199 \\
\hline
\end{tabular}

Mit einer relativen Populationsgröße im Folgejahr von über 100 Brutpaaren können Goldammer, Dorngrasmücke, Heckenbraunelle und Zilpzalp ihre Populationsgröße halten. Das gilt auch bei einer Berechnung der Populationsentwicklung anhand der Angaben über Jahresbruten von SiRIWARDENA et al. (1998). Die Arten erzeugten in den Hecken unter Annahme der für Mitteleuropa geltenden Verhältnisse Populationsüberschüsse von 10 \% (Dorngrasmücke) bis $60 \%$ (Zilpzalp).

Amsel, Singdrossel, Mönchs-, und Gartengrasmücke erreichten in Hecken nur zwischen 77 und $87 \%$ der Populationsgröße des Vorjahres. Unter Annahme einer geringeren Zahl von Jahresbruten $\left(\mathrm{P}_{(\mathrm{t}+1)}\right.$ mit $\left.\mathrm{B}_{(\mathrm{S})}\right)$ erreichte die Klappergrasmücke sogar nur noch $65 \%$ des vorjährigen Populationsniveaus.

Die Berechnung der Populationsgröße im Folgejahr nach Mayfield-Überlebenswahrscheinlichkeiten $\left(\mathrm{P}_{(\mathrm{t}+1)[\text { Mayfield] }}\right)$ führte $\mathrm{im}$ Vergleich $\mathrm{zu} \quad \mathrm{P}_{(\mathrm{t}+1)}$ bei Amsel, Singdrossel, Gartengrasmücke und Heckenbraunelle zu übereinstimmenden Resultaten (Tab. 35). Für den Zilpzalp errechnet sich nach Mayfield eine um $20 \%$ größere Population im Folgejahr. Die Population der Dorngrasmücke würde um $13 \%$ kleiner sein. Das hat zur Folge, dass die Population der Dorngrasmücke geringfügig ab- statt zunimmt.

\subsection{Ursachen für Brutverluste}

Die Mehrzahl der Nestverluste ist auf Prädation zurückzuführen, über die Hälfte aller angefangenen Nester ging dadurch verloren (vgl. Tab. 29). 
Die Brutverluste durch Prädation unterschieden sich artspezifisch (Tab. 36). Bei Amsel, Singdrossel und Klappergrasmücke gingen zwischen 70 bis $78 \%$ verloren. Die Heckenbraunelle verlor über $60 \%$ ihrer Nester durch Räuber. Die geringsten prädationsbedingten Verluste waren bei Zilpzalp, Dorngrasmücke und Gartengrasmücke festzustellen.

Tab. 36: Brutverluste durch Prädation und sonstige Ursachen an acht Singvogelarten in Hecken.

\begin{tabular}{|l|c|c|c|c|c|}
\hline & $\mathrm{N}$ & Prädation & Präd [\%] & sonstige Verluste & sonst. [\%] \\
\hline Amsel & 36 & 28 & 77,7 & 3 & 8,3 \\
Singdrossel & 24 & 17 & 70,8 & 3 & 12,5 \\
Goldammer & 45 & 21 & 46,6 & 5 & 11,1 \\
Dorngrasmücke & 34 & 9 & 26,7 & 8 & 23,5 \\
Mönchsgrasmücke & 22 & 11 & 50 & 3 & 13,6 \\
Gartengrasmücke & 21 & 7 & 33,3 & 7 & 33,3 \\
Klappergrasmücke & 7 & 5 & 100 & 0 & 0 \\
Heckenbraunelle & 19 & 12 & 63,2 & 3 & 15,8 \\
Zilpzalp & 10 & 2 & 20 & 1 & 10 \\
\hline
\end{tabular}

Ein interspezifischer Vergleich der Nesthöhen zeigt, dass Nester von Arten, die häufiger ausgeraubt werden, im Durchschnitt signifikant höher angelegt waren $\left(\mathrm{r}_{\mathrm{s}}=0,881, \mathrm{~N}=8\right.$, $\mathrm{p}<0,01)$. Intraspezifisch betrachtet ist dieser Effekt durch die Nesthöhe nicht zu finden, denn erfolgreiche Nester waren nicht signifikant niedriger angelegt (U-Tests n.s. für acht Singvogelarten: Amsel, Singdrossel, Goldammer, Dorn-, Garten- und Klappergrasmücke, Heckenbraunelle und Zilpzalp). Bei der Mönchsgrasmücke waren dagegen höher angelegte Nester erfolgreicher als niedrige ( $\mathrm{p}<0,05, \mathrm{~N}=15$, U-Test, zweiseitig). Bei der Goldammer lag der Mittelwert für erfolgreiche Nester bei einer Höhe von 46,8cm ( $\mathrm{S}=45)$, der für ausgeraubte Nester bei 29,4 ( $\mathrm{S}=22,4$; U-Test, n.s., $\mathrm{N}=35$ ). Die Erstbrut der Goldammer war weniger erfolgreich die Gelege in der zweiten Brutphase ( $\mathrm{p}<0,05, \mathrm{~N}=45, \mathrm{Z}$-Test, zweiseitig). Im April und Mai resultierte aus sechs erfolgreichen Nestern (Nesterfolg 27,2\%) ein Ausfliegeerfolg von 0,72 Jungvögeln pro Nest. Im Juni und Juli erhöhte sich der Ausfliegeerfolg auf 1,87 (Nesterfolg 56,5\%, $\mathrm{N}=23$ ).

Im April und Mai werden Nester der Goldammer in geringerer Höhe angelegt als zu späteren Zeitpunkten (Abb. 26). 


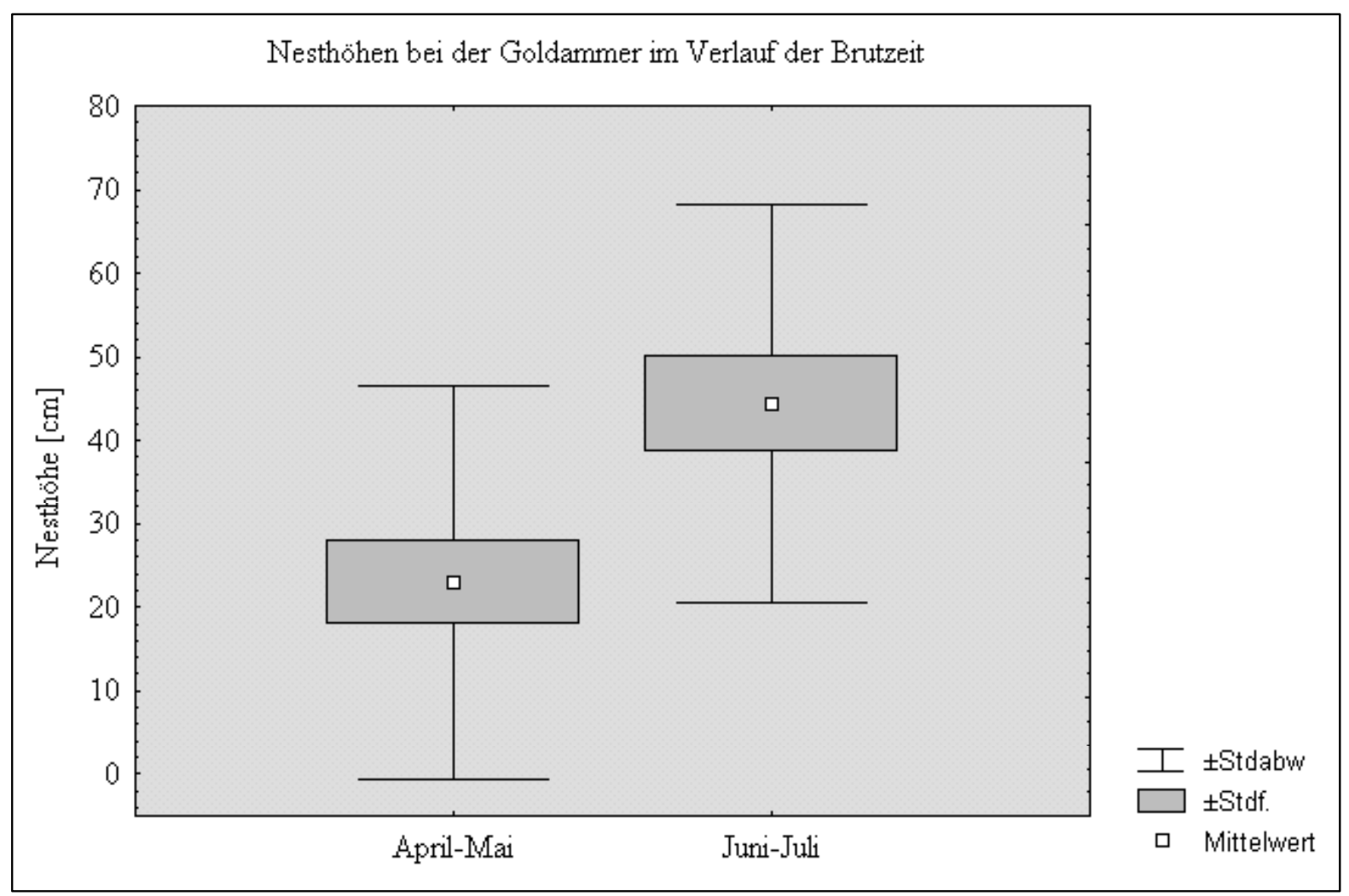

Abb. 26: Nesthöhe bei der Goldammer in Abhängigkeit von der Jahreszeit (U-Test, p< 0,02, N=41, zweiseitig.

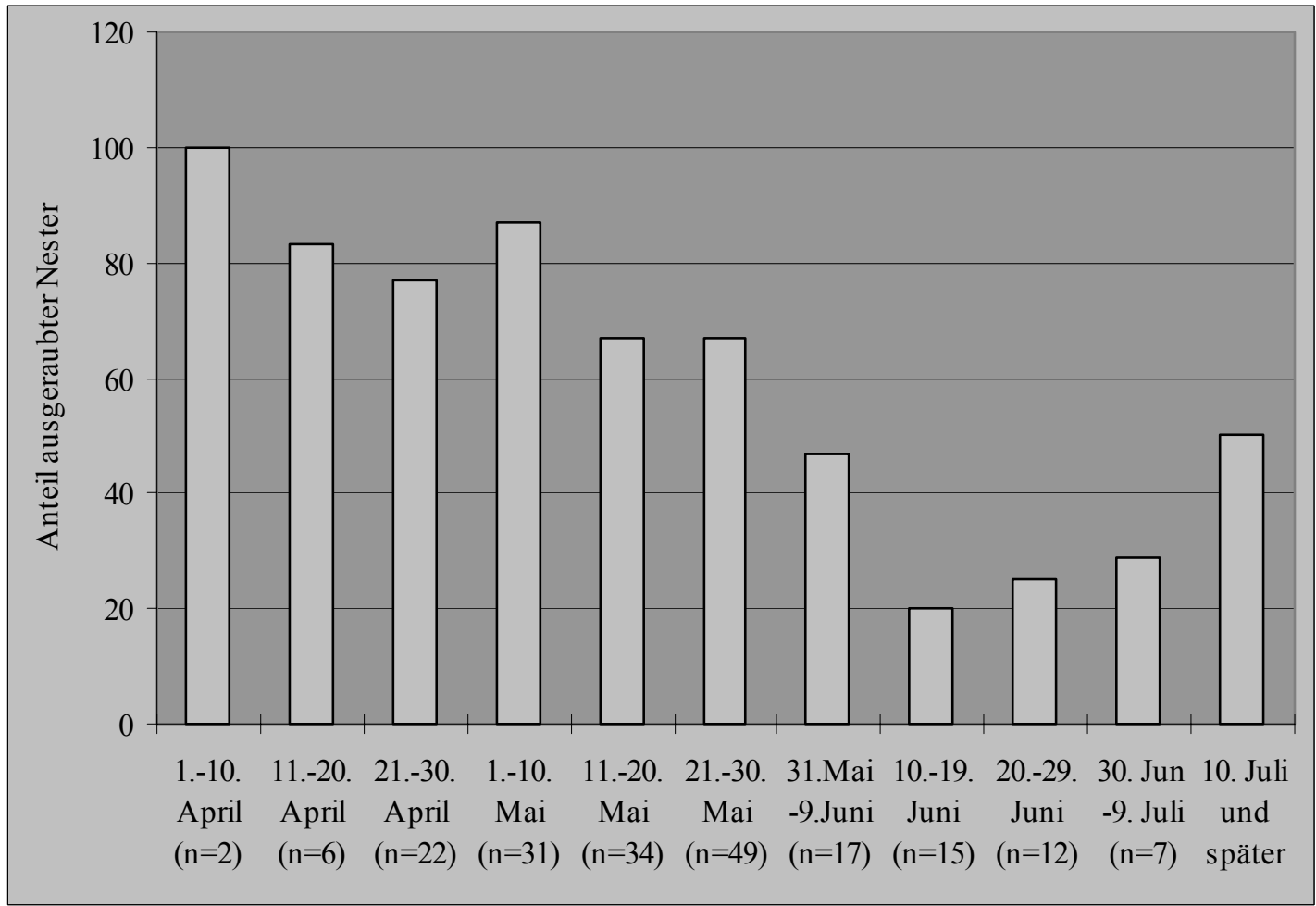

Abb. 27: Zeitlicher Verlauf des Anteils durch Prädation verlorener Nester offen brütender Singvögel. Die Nester sind ihres Fundtages entsprechend datiert.

Im Verlauf der Brutperiode nimmt der Anteil ausgeraubter Nester aller Arten bis Mitte Juni ab und steigt dann wieder an (Abb. 27). Abgesehen von den zwei ausgeraubten Nestern der 
ersten April-Dekade, schwankt die Prädationsrate zwischen der zweiten April- und der ersten Mai-Dekade um eine Rate von 80 \% und ist in diesem Zeitraum an höchsten.

Die Nestverluste durch Prädation waren bei monatlicher Betrachtung bei den im April gefundenen Nestern mit $80 \%$ am höchsten. Von den Nestern im Mai wurden immer noch $73 \%$ ausgeraubt, im Juni und Juli angelegte Nester wurden nur noch zu 32 bzw. $36 \%$ ausgeraubt.

Die Zeitspanne zwischen der Ablage des ersten Eies und dem Zeitpunkt des Nestverlustes durch Prädation kann zwischen wenigen bis über 30 Tagen liegen. Es macht daher Sinn, die Ausraubzeitpunkte der Nester soweit möglich festzustellen, um herauszufinden, wann die meisten Nester ausgefressen werden. Für jedes ausgeraubte Nest wurde ein gemitteltes Datum aus dem Zeitraum der letzten Kontrolle und der Feststellung des Verlustes berechnet. In Tab. 37 sind die Anteile gefundener und ausgeraubter Nester für die Monate der Brutzeit gegenübergestellt.

Tab. 37: Vergleich der prozentualen Verteilung gefundener und ausgeraubter Nester in der Brutzeit.

\begin{tabular}{|c|cc|cc|c|}
\hline & \multicolumn{2}{|c|}{ gefunden } & \multicolumn{2}{c|}{ ausgeraubt } & Vgl. relativer \\
& $\mathrm{N}$ & $\%$ & $\mathrm{~N}$ & $\%$ & Häufigkeit $[\mathrm{p}]$ \\
\hline April & 30 & 15 & 24 & 19 & 0,348 \\
Mai & 114 & 58 & 83 & 67 & 0,107 \\
Juni & 44 & 22 & 14 & 11 & $0,013^{*}$ \\
Juli & 9 & 5 & 3 & 2 & 0,174 \\
\hline
\end{tabular}

Die relative Häufigkeit gefundener und ausgeraubter Nester unterscheidet sich im Juni signifikant (Tab. 37). Es werden weniger Nester ausgeraubt, als nach dem Anteil tatsächlich kontrollierter Nester zu erwarten wäre. Im Mai werden die meisten Nester angelegt und auch die Anzahl ausgeraubter Nester ist am höchsten. Damit zeigt der Räuberdruck auf Nester offen brütender Singvögel eine Dichteabhängigkeit im Mai und Juni: Wenn die meisten Nester vorhanden sind, wird auch der größte Anteil ausgeraubt, geht das Beuteangebot in Form von Nestern im Juni zurück, vermindert sich die Ausraubrate.

Die Zahl der Nestfunde ist im Mai am höchsten, mit 49 Nestern wurden in der dritten Maidekade die meisten Nester gefunden (Abb. 27). Somit kann davon ausgegangen werden, dass Ende Mai die höchste Anzahl von Nestern in Hecken vorhanden war, wenn man die Fundquote aus regelmäßiger und systematischer Nestersuche zu Grunde legt.

Neben Prädation waren Störungen und das Ausmähen von heckenbegleitender krautiger Vegetation weitere Verlustursachen für Bruten. Von Mahd sind sehr stark Dorngrasmücke, 
aber auch Goldammer betroffen (Tab. 38), des Weiteren wurde je ein Nest von Gartengrasmücke, Mönchsgrasmücke und Sumpfrohrsänger umgemäht.

Tab. 38: Anteil der Nestverluste bei Dorngrasmücke ( $N=17$ Verluste gesamt) und Goldammer $(\mathrm{N}=24)$ durch Störungen und das Ausmähen von Krautvegetation an Hecken.

\begin{tabular}{|l|c|c|}
\hline & Mahd & Störung \\
\hline Dorngrasmücke & 23,5 & 11,8 \\
Goldammer & 8,3 & 12,5 \\
\hline
\end{tabular}

In drei Fällen (bei Dorn-, Garten- und Mönchsgrasmücke) wurde das Nest 'nur' freigemäht. Die Nester wurden unmittelbar danach aufgegeben, obwohl die Gelege unbeschädigt waren.
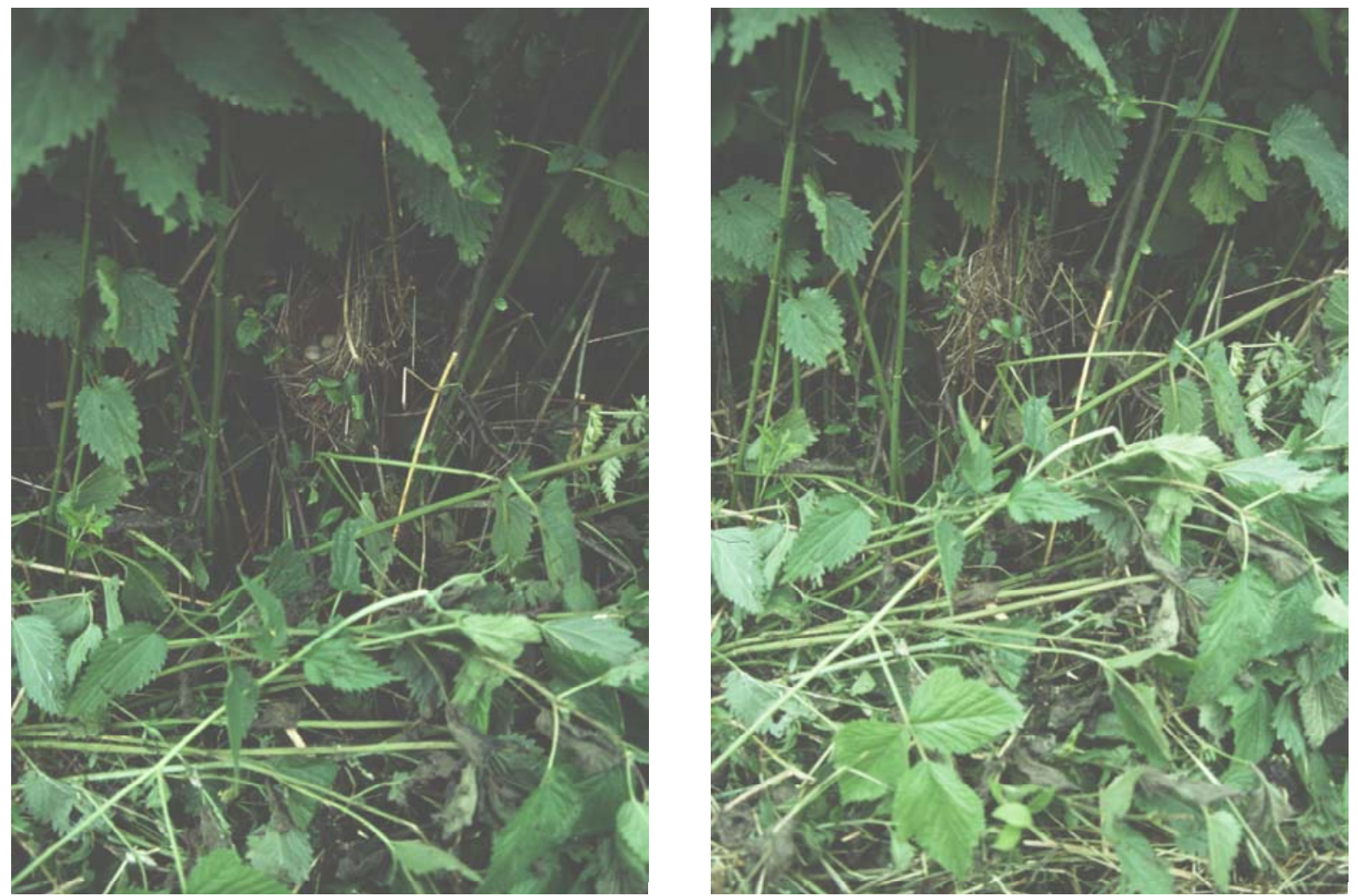

Abb. 28: Freigemähtes Nest der Dorngrasmücke am 27. Mai 1999 (links) und zwei Tage später ausgeraubt (rechts).

Die durch Störungen verursachten Aufgaben von Gelegen (kontrollierte Nester mit Jungvögeln wurden nicht aufgrund von Störungen aufgegeben) lagen bei Goldammer und Dorngrasmücke jeweils über $10 \%$. Somit betrugen die anthropogen bedingten Nestverluste bei diesen Arten über $20 \%$ (Goldammer) bis über ein Drittel aller Nestverluste (Dorngrasmücke). 


\subsection{Prädationsmuster}

\subsubsection{Welche Prädatoren treten an den Kunstnestern auf?}

Anhand der Fotonester wurden Eichelhäher und Elster am häufigsten als Prädatoren an den Kunstnestern festgestellt (Abb. 29, Tab. 39). In jeweils einem Fall wurden auch Feldsperling, Iltis und Rabenkrähe festgestellt. Mäuse traten erst als Nesträuber auf, als ich versuchsweise unbefruchtete Eier von Feldsperlingen in den Fotonestern benutzt habe. Die Schalen der Wachteleier waren für Mäuse nicht zu knacken. Einige dieser Eier zeigten überall Nagespuren auf der Schale, waren aber nicht geöffnet, was ebenfalls darauf hinweist, dass Mäuse als Nesträuber an den in dieser Studie benutzen Kunstnestern ausscheiden.

Tab. 39: Anzahl der ausgeraubten Kunstnester mit Kamera durch Elster und Eichelhäher.

\begin{tabular}{|lcc|}
\hline & Elster & Eichelhäher \\
\hline April-Mai 1999 & 14 & 4 \\
April-Mai 2000 & 4 & 0 \\
\hline ges. April-Mai & 18 & 4 \\
\hline Juni-Juli 1999 & 3 & 10 \\
Juni-Juli 2000 & 2 & 4 \\
\hline ges. Juni-Juli & 5 & 14 \\
\hline Summe & 23 & 18 \\
\hline
\end{tabular}
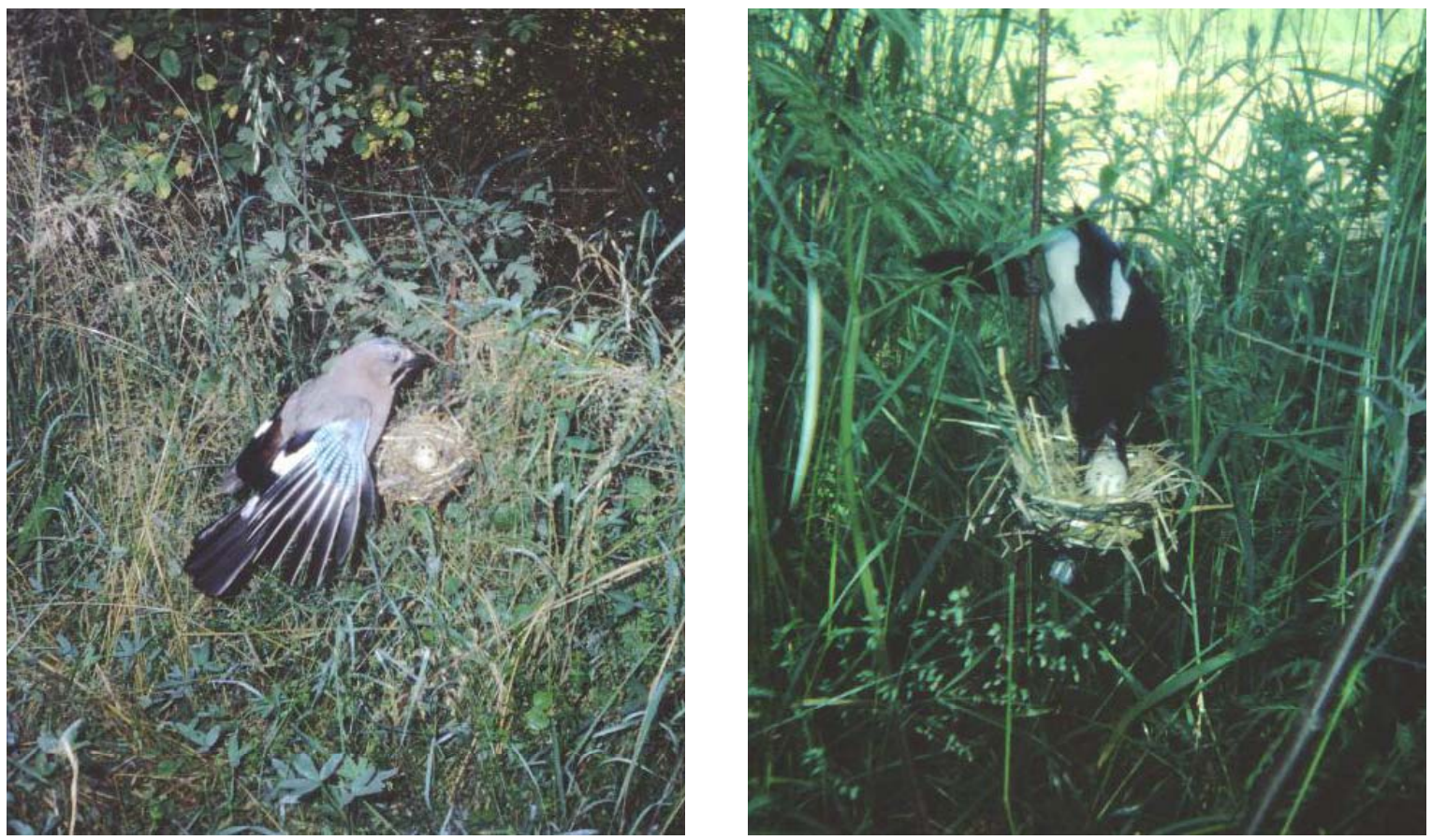

Abb. 29: Eichelhäher und Elster als Nesträuber an Kunstnestern.

Elster und Eichelhäher weichen nicht in der Gesamtzahl ausgeraubter Nester voneinander ab, unterscheiden sich aber signifikant darin, dass die Elster vor allem im April und Mai, der 
Eichelhäher häufiger im Juni und Juli an den Kunstnestern festgestellt wird (Chi²-Exact-Test mit $\chi^{2}=12,75, \mathrm{p}=0,0004, \mathrm{FG}=1$, zweiseitig).

\subsubsection{Wann in der Brutzeit werden die meisten Nester ausgeraubt?}

Von den insgesamt 916 ausgebrachten Kunstnestern 1998 wurden 158 (17\%) nach drei bzw. 316 (35\%) nach sieben Tagen ausgeraubt (Tab. 40).

Tab. 40: Anzahl und Anteil leerer Kunstnester nach drei bzw. sieben Tagen (d) Expositionszeit. Mit * gekennzeichnete Werte weichen nach Z-Test signifikant von den Anteilen leerer Nester in den anderen Zeiträumen ab (* entspricht jeweils p $<0,01)$.

\begin{tabular}{|r|ccc|cc|}
\hline & $\mathrm{N}$ & nach 3d leer & $\%$ & nach 7d leer & $\%$ \\
\hline 4.-8. Mai & 377 & 21 & $6^{*}$ & 143 & 38 \\
18.-22. Mai & 377 & 94 & 25 & 109 & $29 *$ \\
16.-20. Juni & 162 & 43 & 27 & 64 & 40 \\
\hline gesamt & 916 & 158 & 17 & 316 & 35 \\
\hline
\end{tabular}

Die Ausraubung der Nester erfolgte nicht kontinuierlich. Anfang Mai wurden bei der ersten Ausbringung von Kunstnestern nach drei Tagen signifikant weniger Eier gefressen als zu späteren Zeitpunkten. Nach einer Woche Expositionszeit zeigte sich, dass die Verlustrate an Kunstnestern in der zweiten Maihälfte am geringsten war (Tab. 40).

\subsubsection{Welche Nester werden ausgeraubt?}

Von 496 ausgebrachten Kunstnestern an 19 verschiedenen Hecken waren 167 (33,7\%) nach sieben Tagen ausgeraubt (Nester aus Replikation nicht in Analyse). Anhand des Modells konnten unter Aufnahme von vier unabhängigen Variablen 'Heckenbreite', 'Heckenhöhe', 'Saumhöhe' und 'Vegetation um das Nest' 68,5 \% der Fälle klassifiziert werden.

Tab. 41: Ergebnis der schrittweise vorwärts durchgeführten binären Logistischen Regression, mit $N=496$, $\mathbf{F G}=4, p=0,001$.

\begin{tabular}{|l|ccccc|}
\hline & Regr.-koeff. B & SF & Wald-Chi $^{2}$ & Odds Ratio (Unit) & $\mathrm{p}$ \\
\hline Heckenbreite & $-0,239$ & 0,050 & 23,22 & 0,787 & 0,000 \\
Heckenhöhe & 0,267 & 0,060 & 19,50 & 1,306 & 0,000 \\
Saumhöhe & 0,502 & 0,136 & 13,67 & 1,652 & 0,000 \\
Vegetation um Nest & 0,300 & 0,135 & 4,95 & 1,350 & 0,026 \\
\hline Konstante & $-1,933$ & 0,418 & 21,412 & 0,145 & 0,000 \\
\hline
\end{tabular}

Das Ergebnis der Logistischen Regression (Tab. 41) ist so zu interpretieren, dass in breiten Hecken weniger Kunstnester ausgefressen werden als im schmalen. Sind Hecke und Krautsaum in der Nähe des Neststandortes hoch aufgewachsen, so werden Nester eher ausgeraubt, ebenso wenn die Vegetation um das Nest dicht ist. 
In Abb. 30 ist dargestellt, wie sich die Ausraubrate an Kunstnestern in Abhängigkeit zur Heckenbreite verändert.

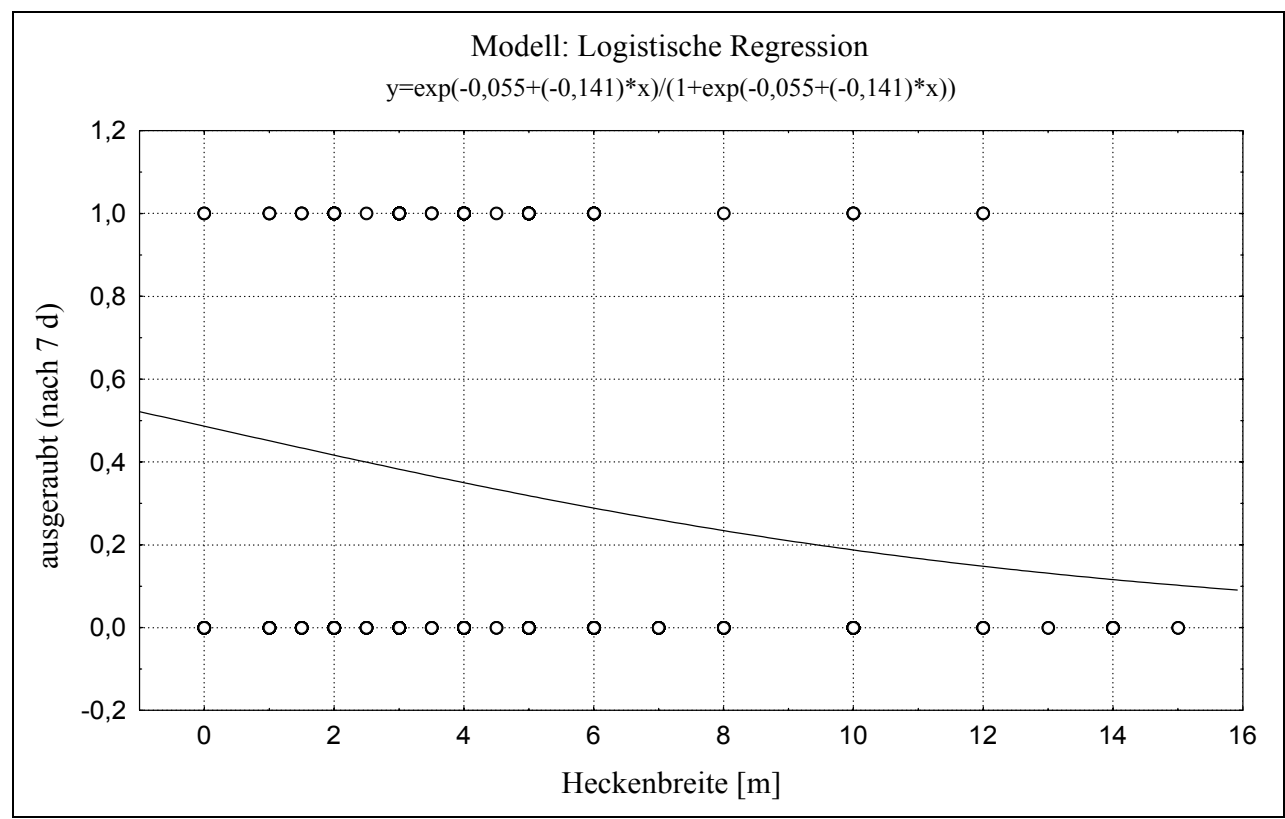

Abb. 30: Zusammenhang zwischen Heckenbreite und Ausraubrate von Kunstnestern nach sieben Tagen Expositionszeit.

In zwei Meter breiten Hecken werden über $40 \%$ und bei 10 Meter Breite nur noch $20 \%$ der Kunstnester ausgefressen (Abb. 30).

\subsubsection{Werden Nester 'heckenweise' ausgeraubt?}

Aus der räumlichen Anordnung der Nester und dem Befund, ob ein Nest nach sieben Tagen ausgeraubt worden ist, wurde eine neue unabhängige Variable 'Nachbar ausgeraubt' generiert, um zu testen, ob Prädatoren ungeachtet der unmittelbaren Neststandortfaktoren, in einigen Hecken Nester ausrauben und in anderen nicht.

Die Wahrscheinlichkeit für ein Nest gefunden und ausgefressen zu werden war hoch (annähernd $70 \%$ ), wenn eines der benachbarten Nester gefunden wurde. Dagegen wurden weniger als $10 \%$ ausgefressen, wenn keines der benachbarten Nester gefunden wurde (Abb. 31). Variablen zur Beschreibung der Exposition eines Nests wurden nicht in der Analyse verwendet, denn die Exposition der Nester änderte sich in der Regel mit einem Wechsel der Hecke. In dieses Bild passt, dass nordexponierte Nester eine ähnlich hohe Signifikanz zeigten, wie südexponierte Nester, die jeweils auf der anderen Seite der Hecke ausgefressen worden waren. Das gleiche Bild zeigte sich für west- bzw. ostexponierte Nester, so dass davon ausgegangen werden musste, dass nicht primär die Exposition der Nester für die Klassifikation von Bedeutung war. 


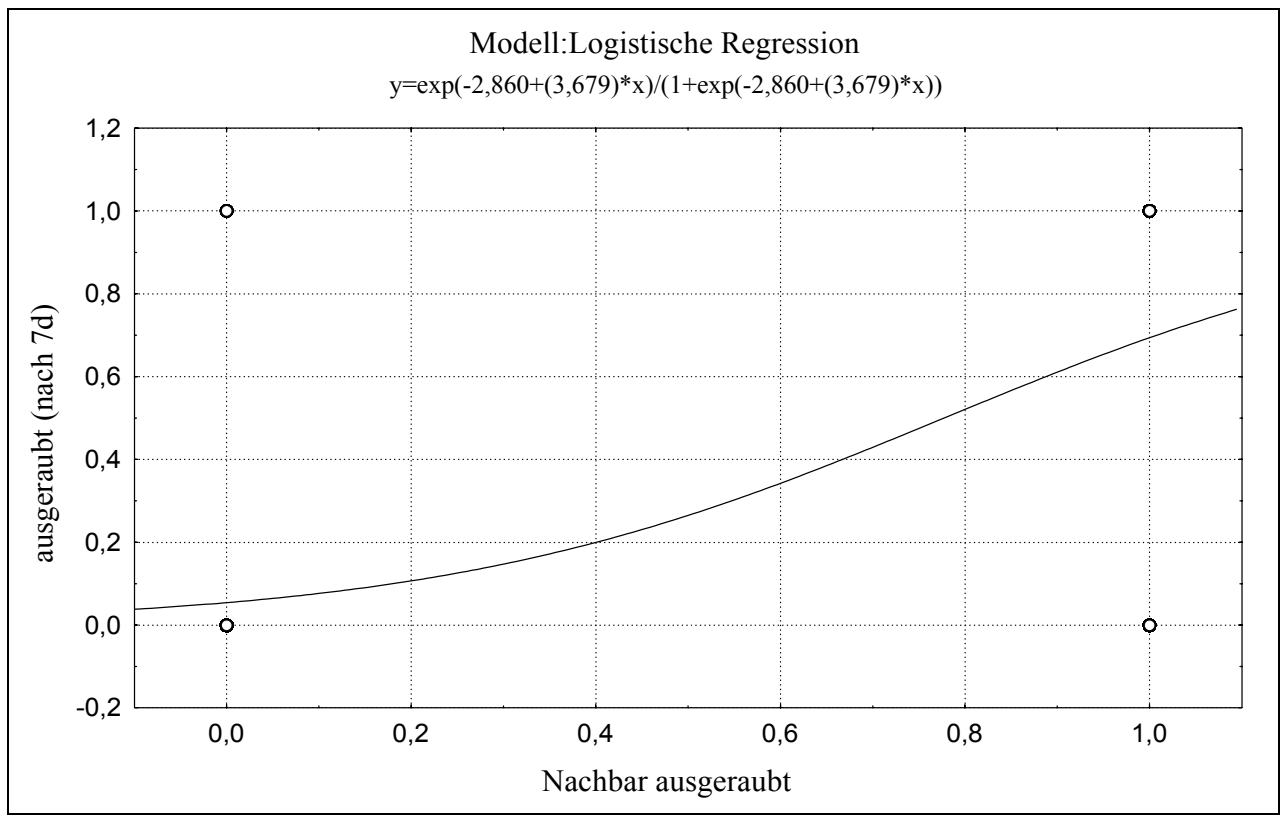

Abb. 31: Beziehung der Ausraubwahrscheinlichkeit eines Kunstnestes in Abhängigkeit des Schicksals der Nachbarnester.

\subsubsection{Welche Landschafts- und Heckenstrukturen bestimmen das Prädationsrisiko von Kunstnestern in Hecken?}

Von 41 Hecken, in denen jeweils sechs Nester für eine Woche exponiert waren, wurden 20 $(48,8 \%)$ vollständig ausgeraubt. In drei Hecken blieben alle Eier erhalten. Das insgesamt signifikante Modell der Logistischen Regression mit 61 \% korrekt klassifizierten Fällen kam sowohl bei einer schrittweise vorwärts als auch schrittweise rückwärts durchgeführten Analyse zu der Aufnahme der Variablen Wegedichte, Waldnähe und Mantel (Tab. 42).

Tab. 42: Ergebnis der schrittweise vorwärts durchgeführten binären Logistischen Regression, mit $N=41$, $\mathbf{F G}=\mathbf{3}, \mathbf{p}=\mathbf{0 , 0 0 7}$.

\begin{tabular}{|l|cccc|}
\hline & Regr.-koeff. B & SF & Wald-Chi & p \\
\hline Wegedichte & 0,004 & 0,002 & 4,50 & 0,34 \\
Waldnähe & 0,003 & 0,002 & 3,63 & 0,57 \\
Mantel & $-0,247$ & 0,148 & 2,78 & 0,96 \\
\hline Konstante & $-1,586$ & 1,024 & 2,40 & 0,12 \\
\hline
\end{tabular}

Das Risiko für Nester in einer Hecke ausgeraubt zu werden ist hoch, wenn die Wegedichte um die Hecke hoch ist, die Entfernung zum nächsten Wald groß ist und wenn der Gebüschmantel der Hecke schmal ist. 


\section{Diskussion}

\subsection{Brutvogelkartierung}

Die in den 30 Hecken erfasste Artenzahl umfasst $14 \%$ der 288 Brutvogelarten Deutschlands (WITT et al. 1996). Das Artenspektrum setzt sich aus Arten lichter Waldhabitate, naturnaher Waldränder und halboffener Landschaften mit Sträuchern und Einzelbäumen zusammen (RINGLER et al. 1997).

Detaillierte Aufzeichnungen über die Vogelwelt in schleswig-holsteinischen Knicks in der Nähe Hamburgs von DIETRICH (1903) bieten eine Vergleichsmöglichkeit der Brutvogelbestände über einen Zeitsprung von etwa 100 Jahren. Der Autor ordnete die häufigsten 13 Arten entsprechend ihrer Dominanz und fügte diesen weitere 24 in abfallender Häufigkeit an, ohne weitere Dominanzwerte zu nennen.

Mit Dorngrasmücke (11,2\%), Goldammer (10\%), Gartengrasmücke (8\%) und Amsel $(7,4 \%)$ waren damals fast die gleichen Arten dominant wie heute. Nur die Heckenbraunelle erreichte mit 4,2 \% halb so hohe Dominanzwerte in den Hecken wie heute um Göttingen (Tab. 24). Neuntöter (6,7 zu heute 1,2\%) und Elster (5,1 zu 1,4\%) weisen dagegen einen beträchtlichen Rückgang ihrer relativen Häufigkeit in Hecken auf. Ebenso fand DIETRICH (1903) für Gelbspötter (4,5 zu 0,32\%) und Baumpieper (4,0 zu 0,68\%) erheblich höhere Dominanzen in den Knicks von Schleswig-Holstein. Bei Fitis (4 zu 1,2 \%), Bluthänfling (4 zu $1,8 \%$ ) und Sumpfrohrsänger (4 zu 2,4\%) sind die Unterschiede vergleichsweise gering.

DIETRICH (1903) gibt für die dominierenden Buscharten in den Knicks Brombeere, Haselstrauch, Eiche, Weide und Weißdorn, mit jeweils in über $75 \%$ der Knicks vorkommend, an. Die um Göttingen dominante Schlehe ist bei DIETRICH (1903) erst an 13. Stelle genannt und kommt in weniger als der Hälfte aller Knicks vor. Rose und Holunder waren nach Dietrich noch seltener (unter $25 \%$ ). Insofern unterscheiden sich die von DIETRICH (1903) und die in dieser Studie untersuchten Hecken aufgrund ihrer Gehölzartenflora erheblich. Über die Strukturen der Knicks finden sich in der Arbeit keine Anhaltspunkte, außer dass nach der Rodung von Knicks auch Überhälter stehen gelassen wurden, vor allem Eichen, die in 39 \% der Hecken als Baum festgestellt werden konnten. Insofern kann davon ausgegangen werden, dass alte Knicks in Schleswig-Holstein vor 100 Jahren in ihrer Struktur ähnlich aufgebaut waren, wie die von mir untersuchten Hecken um Göttingen, dass die Knicks aber durch häufige Nutzung durchschnittlich viel jüngeren Bewuchs aufwiesen und eine höhere Artenzahl an Gehölzpflanzen beherbergten. 
In den Hecken um Göttingen ist das 'auf den Stock setzten', der Rückschnitt von Hecken auf etwa 10-20 cm Stocklänge über dem Boden, keine übliche Pflegemethode. Die Hecken werden in unregelmäßigen Abständen an den Seiten abgeschlegelt und der Krautsaum wird zumeist jährlich gegen Ende Mai bis Mitte Juli gemäht. Zum einen kann damit die Gehölzzusammensetzung und die Dominanz der Schlehe erklärt werden, zum anderen die höhere Dichte von Arten wie Heckenbraunelle oder Mönchsgrasmücke. Letztere zählte DIETRICH (1903) zu den selten oder nur ausnahmsweise auftretenden Arten. Bemerkenswert ist das Fehlen der Singdrossel bei Dietrich, eine Art die PUCHSTEIN (1980) in Schleswig-Holstein als subdominant einstufte und die in Göttingen in über 2/3 der untersuchten Hecken brütete (Tab. 22). Dietrich (1903) fand insgesamt weniger als $10 \%$ Baumbrüter in den Hecken, so dass das Fehlen der Singdrossel eventuell auf das Fehlen geeigneter Nistorte zurückgeführt werden kann, zumal er die Amsel nach seinen Beobachtungen in den Hecken als 'Erdnister' bezeichnete.

Der besondere Wert der historischen Arbeit von DIETRICH (1903) ergibt sich aus der Vergleichsmöglichkeit des Arteninventars, auch wenn die Hecken in ihrer Ausprägung und Nutzung zu den von mir untersuchten Hecken Unterschiede aufweisen und auch davon auszugehen ist, dass die Landnutzung gravierende Veränderungen erfahren hat (vgl. dazu GeORge 1996a, Busche 1997, Evans 1997). Zwar ist die Artenzahl der Vögel in Hecken um Göttingen heute höher, doch listet DIETRICH (1903) neun Vogelarten auf, die 100 Jahre später nicht mehr als Heckenbrutvögel festgestellt werden können: Gartenrotschwanz, Nachtigall, Braunkehlchen, Raubwürger, Saatkrähe, Wendehals, Grauschnäpper Sperbergrasmücke und Grauammer, nach ihrer Häufigkeit sortiert. Fünf dieser Arten waren bereits in den Untersuchungen von HAHN (1966), KIRCHHOFF \& IHSSEN (1972) und PUCHSTEIN (1980) in Knicks nicht mehr nachweisbar. Zu dem Zeitpunkt der letztgenannten Untersuchungen hatten bereits großflächige Rodungen von Hecken zu Gunsten eines Strukturwandels in der Landwirtschaft eingesetzt (z.B. BEZzEL 1982). In den von mir bearbeiteten Hecken waren zum Teil noch Heckenstrukturen erhalten, die "in ihrer Form so schon vor dem II. Weltkrieg existierten" (mdl. Mitteilung von Herrn Evers, Landwirt und Verwahrer der historischen Feldkarten der Feldmarksinteressentenschaft in Volkerode). Gartenrotschwanz, Nachtigall, Braunkehlchen, Raubwürger, Grauschnäpper und Grauammer konnten aber jeweils nur an ein bis maximal drei Tagen festgestellt werden, nach über 300 Beobachtungstagen. Wendehals, Saatkrähe und Sperbergrasmücke traten in den von mir untersuchten Hecken nicht auf.

Die Populationsentwicklung der Dorngrasmücke zeigte einen starken Bestandseinbruch gegen Ende der sechziger Jahre (BERTHOLD 1973, 1974), der sich auf ganz Europa erstreckte 
(Winstanely et al. 1974 (aus Boddy 1993). Danach konnte sich die Population zwar wieder stabilisieren, allerdings auf niedrigerem Niveau (MARCHANT et al. 1990, BAUER \& BERTHOLD 2000). Die Dominanz der Dorngrasmücke in Hecken spiegelt die Bestandsentwicklung über die letzten 100 Jahre wider: Um 1900 bis in die 60er Jahre hinein ist die Art der häufigste Heckenvogel Norddeutschlands und die Dominanz liegt zwischen 11,2 (um 1900) bis 17,3 (1966) Prozent (Dietrich 1903, Hahn 1966, KirChHOFF \& IHSSEn 1972, PuChSTEIN 1980). 1974 weisen die Daten von PUChSTEIN (1980) dann einen Einbruch der DorngrasmückenBestände aus: Die Dominanz liegt nur noch bei 8,2 \%. In den Untersuchungsgebieten um Göttingen ist die Art über die Untersuchungsjahre konstant mit 7,7 \% vertreten. Mit Goldammer, Heckenbraunelle und Amsel sind in den Hecken drei Arten häufiger (Tab. 24). Für den Landkreis Göttingen stellt DöRRIE (2000) fest, dass die Dorngrasmücke "in geeigneten Habitaten wieder ein durchaus häufiger Vogel ist". Der Vergleich mit den historischen Daten zeigt aber, dass auch diese heute wieder relativ häufige Art bis vor 35 Jahren noch erheblich höhere Dichten gehabt haben könnte: HEITKAMP (1981) fand in seinen Untersuchungen an einer Saumbiozönose bei Göttingen zwischen 1963-1967, dass die Dorngrasmücke in der offenen Gebüschlandschaft „dominant als zweithäufigste Art hinter der Goldammer“ mit $11,1-17,8 \mathrm{BP} / 10$ ha auftrat.

Eine Zunahme der relativen Häufigkeit war bei Heckenbraunelle (von 4,2 \% bei DIETRICH (1903) auf 8,5 \% in dieser Studie) und Goldammer (von $10 \%$ auf 12,9\%) festzustellen. Die Zunahme weiterer Arten kann nicht exakt dokumentiert werden, weil Dietrich für seltenere Arten keine Dominanzwerte angegeben hat. Er bezeichnet aber die heute sehr häufigen Arten Mönchsgrasmücke, Feldsperling, Kohl- und Blaumeise, Zilpzalp und Ringeltaube als 'ziemlich selten oder nur ausnahmsweise' in Hecken brütenden Vögel. Die Dominanz der Heckenbraunelle ist mit 8,5 $\mathrm{zu}$ 4,2 heute doppelt so hoch und die Singdrossel, von der in dieser Studie vier Brutpaare je Kilometer Hecke festgestellt wurden, wird bei DIETRICH (1903) überhaupt nicht erwähnt. Während die hohen Dichten von Feldsperling und zum Teil auch von Kohl- und Blaumeise auf der großen Anzahl von Nistkästen in den Hecken basieren, zeigt das Auftreten der anderen heute häufigen Arten vor allem Auswirkungen der strukturellen Veränderung der Hecken. Die Arten, die in den Hecken um Göttingen häufiger vorkommen als in den schleswig-holsteinischen Knicks, sind alle in bestimmter Weise von Gehölzstrukturen abhängig und weniger von der Nutzung der Flächen um die Hecken. Das ist bei vielen Arten, die in ihren Bestand abgenommen haben, umgekehrt: Neuntöter, Elster, Rebhuhn, Turteltaube, Sumpfrohrsänger, Baumpieper, Bluthänfling und vor allem viele der vollkommen verschwundenen Arten wie Gartenrotschwanz, Braunkehlchen, Raubwürger, 
Saatkrähe, Wendehals, Grauschnäpper und Grauammer benötigen mindestens zum Nahrungserwerb Habitatstrukturen, die über die Hecke hinausgehen. Für diese Arten steht zu vermuten, dass nicht die Nutzungsaufgabe der Heckengehölze, sondern eine andere oder intensivere Nutzung der Heckenumgebung zu den Bestandsabnahmen geführt hat. Auch SIRIWARDENA et al. (2000) haben für Rebhuhn, Turteltaube, Feldlerche, Feldsperling, Bluthänfling und Grauammer starke Bestandsrückgänge zwischen 1969 und 1995 auf eine durch technischen Fortschritt begründete Intensivierung der Landwirtschaft zurückgeführt. Unter den Arten, die rückläufige Bestandszahlen haben, waren mit Gimpel und Singdrossel in England und Wales auch Waldarten sowie die Rohrammer als Feuchtlandbewohnerin betroffen. Die Mehrzahl der abnehmenden Arten war jedoch von den Autoren als 'Farmland Spezialisten' kategorisiert und in erster Linie von den Einflüssen höherer Pestizid- und Düngemitteleinsätze betroffen (SIRIWARDENA et al. 2000).

\subsection{Siedlungsdichte}

Vögel siedeln in Hecken in relativ hohen Dichten. Das zeigt ein Vergleich mit anderen Habitattypen. Für die Siedlungsdichte in Hecken gibt Flade (1994) Werte an, die an die Dichten in Waldtypen heranreichen (Tab. 43).

Tab. 43: Besiedlungsdichte einiger Habitate durch Vögel, nach FLADE 1994.

\begin{tabular}{|lc|}
\hline \multicolumn{1}{|c}{ Habitat } & Siedlungsdichte (BP/10ha) \\
\hline Gehölzarme Felder & 5,3 \\
Wiesen und Weiden & 5,3 \\
Feldgehölze & 11,7 \\
& 19,8 \\
Hecken (halboffene Feldflur) & 28,0 \\
einfache Hecken/Knicks & 43,0 \\
Doppelknicks & 34,4 \\
Berg-Buchenwälder & 55,4 \\
Tiefland-Buchenwälder & \\
\hline
\end{tabular}

Bei einer angenommenen durchschnittlichen Breite von 10 Metern über alle Hecken ergibt sich rein rechnerisch die utopische Revierdichte von $880 \mathrm{BP} / 10$ ha. Eine derart hohe Dichte von Brutpaaren ist nur deshalb möglich, weil viele Arten ihre Nahrung außerhalb der Gehölzstrukturen der Hecke suchen. So liegen etwa $90 \%$ aller Nahrungsflüge der Goldammer innerhalb eines Radius von $250 \mathrm{~m}$ um den Neststandort (LILle 1996). Bei der Berechnung von Siedlungsdichten in Hecken sollte deshalb die Nutzung der unmittelbaren Umgebung durch die jeweils betrachteten Arten einbezogen werden. Unter Berücksichtigung eines 500 
Meter breiten Streifens angrenzender Kulturflächen ergibt sich für alle untersuchten Hecken dieser Studie eine Siedlungsdichte von 18 Brutpaaren auf 10 Hektar.

\subsection{Wie konstant ist die Revierbesetzung in Hecken über die Jahre?}

Die Besetzung von Revieren steht nicht mit dem Bruterfolg in Beziehung (VICKERY et al. 1992c) und ein Rückschluss auf Habitatqualität ist nicht zulässig (VAN HoRNE 1983). Um die unterschiedliche Besetzung von Revieren in Hecken $\mathrm{zu}$ beschreiben, spreche ich deshalb im Folgenden von Habitatattraktivität und bezeichne damit die Etablierung von Revieren in Hecken durch eine Art über die Jahre. Maximale Habitatattraktivität darf nicht mit optimaler Habitatqualität gleichgesetzt werden, aber es ist naheliegend, dass selten besiedelte Hecken auch qualitativ schlechtere Habitate darstellen.

Aufgrund der Ergebnisse der mehrmaligen Revierkartierung können drei Kategorien von Hecken mit unterschiedlicher Habitatattraktivität für eine jeweils $\mathrm{zu}$ betrachtende Art unterschieden werden. Eine über die Jahre konstante Besiedlung einer Hecke oder allgemein eines Habitats kann als Maß für höchste Habitatattraktivität herangezogen werden. Temporär besiedelte Hecken haben nur eine mittlere Attraktivität, während eine unbesiedelte Hecke für eine betreffende Art zu einem definierten Zeitpunkt unattraktiv ist, sofern man davon ausgeht, dass die Population der betrachteten Art ausreichend Individuen umfasst, um freie und geeignete Habitate besetzen zu können. Das ist für die häufigsten 20 Arten dieser Untersuchung und auf das Untersuchungsgebiet bezogen anzunehmen.

PUCHSTEIN (1980) fand in einem Vergleich von Vogelbestandsaufnahmen an Knicks in den Jahren 1961 bis 1974 unter Einbeziehung der Arbeiten von HAHN (1966) und KIRCHHOFF \& IHSSEN (1972) einen „hohen Grad der qualitativen Übereinstimmung” der Ergebnisse mit denen von DIETRICH (1903) und begründete damit seine Schlussfolgerung, dass ,die Vogelwelt der Knicks [...] ein recht stabiles Gefüge" sei. Dieser Eindruck wird auch durch die Befunde in Tab. 20 bestätigt: Die Artzusammensetzungen und Brutpaarsummen weisen über die Untersuchungsjahre nur geringe Veränderungen auf. Wird aber die Konstanz der Besiedlung einer Hecke durch eine Art betrachtet, so ist ein erheblicher Anteil der Hecken nur in einem oder in zwei Jahren der Untersuchung besiedelt (Tab. 22). Das weist auf eine hohe Dynamik innerhalb dieses Habitattyps hin. Da die Struktur der Hecken über den Untersuchungszeitraum bis auf wenige Ausnahmen erhalten geblieben ist, stellt sich die Frage, ob in einzelnen Jahren weniger Vögel vorhanden waren, um alle Reviere zu besetzen. In den von mir untersuchten Hecken war das nicht der Fall: Die Summen aller Reviere wichen zwischen den Jahren um maximal $5 \%$ voneinander ab (Tab. 21). Vor allem die häufigen Arten zeigten 
nur geringe Abweichungen ihrer Bestandszahlen über die Jahre (ebd.). Da aber keine individuelle Kennung der Revierinhaber möglich war, ist nicht auszuschließen, dass während der Brutzeit ein ständiger Sog durch frei werdende Reviere in attraktiveren Habitaten für ein Abwandern einzelner Individuen sorgt (vgl. RIDDINGTON \& GOSLER 1995).

Bei der Betrachtung der kontinuierlichen Besiedlung einzelner Hecken durch Brutvögel, wird deutlich, dass eine hohe Fluktuation der Reviere zwischen den einzelnen Hecken über die Jahre im Untersuchungsgebiet besteht. Weniger als die Hälfte aller Brutpaare, die in den Hecken festgestellt wurden, etablierten ein Revier in einer bestimmten Hecke über alle drei Jahre der Untersuchung (Tab. 22, Anmerkung: Weil die Brutpaare nicht individuell markiert waren, konnten jeweils beliebige und ebenso mehrfach wechselnde Individuen hier bezeichnete Brutpaare bilden). Während viele häufige Arten noch sehr konstant in Hecken auftraten (z.B. Goldammer, Mönchs- und Dorngrasmücke, Amsel und Heckenbraunelle), lag der Anteil der nur zeitweilig besiedelten Hecken bei den subdominanten Arten (2-5\%) schon über $40 \%$. Der Anteil zeitweilig besiedelter Hecken ist bei der Klappergrasmücke mit $84 \%$ sehr hoch und eventuell auf einen starken Bestandsanstieg von 1999 auf 2000 zurückzuführen (Tab. 21). Dagegen traten jahrweise wechselnde Revieretablierungen bei anderen Arten unabhängig von Bestandsfluktuationen auf: Beim Zilpzalp wurden z.B. in jedem Jahr 20 Reviere festgestellt (Tab. 21). Diese verteilten sich auf 15 verschiedene Hecken und nur 9 davon wurden regelmäßig genutzt (Tab. 22), so dass die Art trotz einer konstanten Anzahl von 20 Brutpaaren in $40 \%$ der untersuchten Hecken nicht regelmäßig als Brutvogel auftrat.

Für den Zilpzalp lassen sich 15 Hecken als unattraktiv (in keinem Jahr besiedelt), von 9 Hecken mit maximaler (in jedem Jahr besiedelt) sowie 6 Hecken mit mittlerer (suboptimal, nur in manchen Jahren besetzte Reviere) Attraktivität unterscheiden. $40 \%$ der Hecken, die der Zilpzalp überhaupt besiedelt, müssen als suboptimal gelten (Tab. 22).

Der Anteil der Reviere aller Arten, die in suboptimalen Hecken angelegt sind (Tab. 22), erreicht über $24 \%$. Der Anteil solcher wechselnden Reviere ist von Art zu Art sehr unterschiedlich, wobei die häufigeren Arten die geringsten Anteile suboptimaler Reviere aufweisen. Das ist darauf zurückzuführen, dass häufige Arten oft mehrere Reviere in einzelnen Hecken besetzt haben können, in der die Aufgabe eines Territoriums nicht zum Verlassen der Hecke durch die Art führt (vgl. Definition zur 'Besiedlung oder Aufgabe einer Hecke als Brutort', Tab. 22). Zudem kann bei häufigen Arten der Populationsüberschuss so groß sein, dass frei werdende Reviere sofort wieder besetzt werden.

Vor allem bei den Arten, die bereits aufgrund von Bestandsrückgängen oder regionaler Seltenheit auf den Roten Listen geführt werden (z.B. Feldschwirl, Kuckuck, Turteltaube), 
kann eine dauerhafte Besiedlung einer Hecke auch durch überregionale Auswirkungen von Populationsschwankungen beeinflusst sein. Die Habitate mit höchster Attraktivität sollten aber die letzten sein, die aufgegeben werden.

Arten in deren Home-Range mehrere Hecken eingebunden waren, wurden entsprechend ihres Neststandortes einer Hecke zugeordnet. Die Elster wechselte zur Anlage eines neuen Nests in vielen Fällen auch die Hecke, wobei sich die Anzahl der Brutpaare über die Jahre zwischen sechs bis acht relativ konstant hielt, sich aber keine Revierkonstanz in Bezug auf eine Hecke ergab. Das Problem der Revierzuordnung bestand in ähnlicher Weise auch für Neuntöter, Eichelhäher, Rabenkrähe, Waldohreule, Kernbeißer, Bunt- und Kleinspecht und insbesondere für den Kuckuck.

Unter anderen Umständen oder Fragestellungen kann auch die einfache Registrierung von Kontakten und deren Summierung für bestimmte Analysen sinnvoll sein (OSBORNE 1984, PARISH et al 1994).

Das Artenspektrum heckenbewohnender Vögel ist innerhalb Deutschlands sehr unterschiedlich, wenn man die Liste typischer Heckenvögel von Nitsche \& PLACHTER (1987), erweitert in RINGLER et al. 1997) mit dem Artenspektrum in norddeutschen Hecken vergleicht. In Bayern treten mit Zippammer, Ortolan und Schwarzkehlchen Heckenbewohner auf, die zum Teil auf spezielle Attribute wie Streuobst oder feuchte Grünlandbrachen angewiesen sind. Wenn auch nur vereinzelt brütend, erscheinen (oder erschienen) mit Pirol, Wespenbussard, Rot- und Schwarzmilan, Rotkopfwürger, Schwarzstirnwürger, Zaunammer (BLAB 1993) und Baumfalke (RINGLER et al. 1997) weitere Arten in den Hecken Süddeutschlands, für die in der norddeutschen Tiefebene bislang meines Wissens Brutnachweise in Hecken fehlen (abgesehen von einzelnen Feldgehölzbruten der Milane). Auch wenn für Süddeutschland mit Neuntöter, Raubwürger und Rebhuhn nur drei Arten als typische Heckenbewohner angegeben werden (Ringler et al. 1997, vgl. Nitsche \& PlaChter 1987), lassen sich doch viele Arten benennen, die ohne Hecken in der 'siedlungsfernen Agrarlandschaft nicht vorkämen', zum Beispiel Dorn- und Klappergrasmücke, Bluthänfling, Goldammer, Gelbspötter, Ringeltaube und Buchfink (BEZZEL 1982).

Im Untersuchungsgebiet konzentrieren sich die Populationen von Goldammer, Dorngrasmücke, Neuntöter, Feldsperling und der Turteltaube in Hecken (vgl. auch HEITKAMP 1981, DÖRRIE 2000). Baumpieper besetzen im Landkreis Göttingen eher Waldrandhabitate, sofern Waldrandstruktur und die angrenzende Feldwirtschaft (keine dicht stehenden Wintergetreide) und der Wegebau (kein Revier an gepflasterten oder asphaltierten Wegen festgestellt) dies zulassen. Bluthänflinge nutzen eher Ränder und Gehölzlücken in Hecken oder Einzelbüsche, 
wobei die Art auch in den (Rand-) Strukturen von mehrjährigen oder Dauerbrachen brütet. Der Feldschwirl profitiert von Hecken insofern, als dass diese in ihren Gehölzlücken oder in den Randseiten krautige Bereiche vor zu intensiver Pflege oder der Bewirtschaftung schützen und so der Art Brut- und Lebensräume erhalten (Hecken 51, 53 und 70).

\subsection{Besiedlungsdichte und Heckenstrukturen}

In der intensiv bewirtschafteten und nahezu unbewaldeten niedersächsischen Bördenlandschaft nördlich des Harzes zwischen Hannover und Braunschweig (ca. 120 km nördlich der Göttinger Untersuchungsgebiete) fanden OELKE et al. (1992) auf über 2500 ha 42 Brutvogelarten. Die Diversität der Brutvögel in der dortigen Agrarlandschaft betrug $\mathrm{H}_{\mathrm{s}}=1,25$ und war damit doppelt so hoch wie der Diversitätswert einer Vergleichsfläche in der Magdeburger Börde, der zeitgleich und mit gleicher Erfassungsmethode des Brutvogelbestandes erhoben wurde $\left(\mathrm{H}_{\mathrm{s}}=0,62\right.$, OELKE et al. 1992). In einer ebenfalls intensiv bewirtschafteten landwirtschaftlichen Nutzfläche in der Wetterau (bei Hanau, ca. 220km südlich von Göttingen) fand KLEIN (1979) zwischen 1976 und 1978 Werte zwischen $H_{s}=1,4$ und 1,6. Die in allen Untersuchungsgebieten vorhandenen Hecken und Flurgehölze trugen dazu bei, dass die Brutvogeldiversität dieser Landschaften nicht noch geringer war. So fanden OELKE et al. (1992) allein in den Hecken und Gebüschen des oben genannten Untersuchungsgebietes Sommervogelbestände mit 51,4 BP/10 ha, was etwa 8,1 BP/Km entspricht.

PUCHSTEIN (1980) ermittelte in einer schleswig-holsteinischen Knicklandschaft 39 Arten auf 80 ha Untersuchungsfläche mit 20,3 bis 27,6 BP/10 ha. Bei $120 \mathrm{~m}$ Knick je ha sind das 16,9 bis $23 \mathrm{BP} / \mathrm{Km}$. Doppelknicks sind mit doppelter Heckenlänge in die Angaben eingeflossen. Doch selbst wenn die Anzahl der bei Puchstein gefundenen Besiedlungsdichten verdoppelt würden (ausschließlich Doppelknicks vorhanden), blieben die Revierdichten weit unterhalb der in dieser Studie gefundenen Werte von etwa 88 Revieren auf einen Kilometer Heckenlänge. Dabei ist zu beachten, dass der bei PuCHSTEIN (1980) in einigen Jahren häufigste Vogel, die Feldlerche, in der Studie um Göttingen nicht mit erfasst wurde. Die Besiedlungsdichten der von OELKE et al. (1992) untersuchten Hecken in einer niedersächsischen Bördenlandschaft wurden um das Zehnfache übertroffen. Auch die Diversitätswerte der Brutvögel in den Hecken um Göttingen liegen mit $\mathrm{H}_{\mathrm{S}}=3,4$ erheblich höher als die der intensiv genutzten Agrarlandschaften in Nord- und Mitteldeutschland. Die in dieser Studie bearbeiteten Hecken dürften also als überdurchschnittlich artenreich und als sehr dicht von Brutvögeln besiedelt gelten. 
PUCHSTEIN (1980) hebt neben Doppelknicks die Bedeutung von Knickverzweigungen hervor: 100 m Doppelknick wurden von der gleichen Menge Vögel bewohnt, wie 590 m Einzelknick oder drei Knickverzweigungen (bei der jeweils 33 m Knickstrecke ab Verzweigung einbezogen wurde). Unter Berücksichtigung aller an und in Hecken brütenden Vögel, wurden in meiner Untersuchung mit 44 Arten eine höhere Anzahl von Brutvogelarten als von PUCHSTEIN (1980) in einer Knick-Agrarlandschaft (33 Arten) oder HAHN (1966) in einer 'Wiesen-Knick-Landschaft' in Schleswig-Holstein (36 Arten) gefunden. Die höhere Brutvogeldichte dieser Arbeit dürfte auf die erheblich flächigere Struktur der Hecken um Göttingen zurückzuführen sein, denn die in Schleswig-Holstein untersuchten Knicks sind in der Regel 3-5 m breit (z.B. KIRCHHOFF \& IHSSEN 1972), während die in dieser Studie untersuchten Hecken im Mittel 15,8 m breit waren. Die Abhängigkeit der Brutpaardichte von der Heckenbreite ist in Abb. 24 dargestellt und passt mit den Ergebnissen PuCHSTEINS (1980) zur Bedeutung von Doppelknicks und Verzweigungen zusammen. LACK (1988) fand um den Faktor 1,7 höhere Brutpaardichten an T-Verzweigungen als an Kontrollpunkten und begründet das mit den Vorteilen kleinerer und kompakter Reviere mit möglichst kurzem Randbereich, weil solche Reviere besser zu verteidigen seien. Die Wege zu Nahrungsflächen mögen dann kürzer und Schutzmöglichkeiten besser sein, weil Verzweigungen Deckung für mehrere Richtungen bieten. Das Nist- und Rastplatzangebot erhöhe sich und die 'Qualität' der Hecke selbst könne aufgrund der Unzugänglichkeit für Pflegemaßnahmen besser sein. Die ohne tatsächliche Messung der relevanten Parameter diskutierten Ursachen würden nach LACK (1988) auch die höheren Revierdichten in breiten Hecken erklären, sofern die betreffenden Arten eine starke Abhängigkeit von der Gehölzstruktur der Hecke und weniger von deren Umland zeigten.

\subsection{Welche Strukturen und Landschaftselemente bestimmen die Abundanz von Brutvögeln in Hecken?}

Die Abundanz von Brutvögeln wird maßgeblich von der Breite der untersuchten Hecken bestimmt (Tab. 25, Abb. 24). Die Bedeutung dieses Heckenparameters für die Brutpaardichte oder Artenzahl von Heckenvögeln fanden auch Hinsley \& Bellamy (2000) in einer Analyse von 22 Probeflächen in England. In Verbindung mit dem hier untersuchten Parameter 'Anzahl der Überhälter', der als relatives Höhenmaß betrachtet werden kann, steht damit die Bedeutung des (Gehölz-) Volumens einer Hecke vor allen anderen Einflußgrößen (vgl. Tab. 25 und Tab. 26). ARNOLD (1983) und GREEN et al. (1994) fanden übereinstimmend in der Höhe und Breite von Hecken die wichtigsten Parameter für die Brutvogeldichte der meisten Arten und PARISH et al. (1994) bestimmten die 'physikalische Größe' (Länge, Höhe 
und Kronenweite) einer Hecke neben der Anzahl und Höhe der Bäume und einer Korrelation mit angrenzenden Weiden als wichtigste Größen zur Beschreibung der Artenzahl in fünf Untersuchungsgebieten auf insgesamt 79 Transekten.

In der Analyse der 30 Hecken um Göttingen wird ebenfalls ein Einfluss der Länge von Hecken gefunden, jedoch in der Weise, dass kürzere Hecken eine höhere Revierdichte aufweisen (Tab. 25), wohingegen die Artenzahl in Übereinstimmung mit PARISH et al. (1994) mit zunehmender Länge der Hecke ansteigt (Abb. 25).

Die verhältnismäßig hohen Dichten in den kurzen Hecken resultieren daraus, dass mit der Bezugseinheit Hecke nicht für alle Arten das tatsächlich genutzte Habitat quantitativ erfasst wird. Bereits kleinste Heckenfragmente werden von einigen Arten besiedelt, wobei die Reviere eine große Fläche außerhalb der Hecken einschließen. Die Goldammer zum Beispiel kann in 50 m kurzen Hecken vorkommen, was aber nicht bedeutet, dass auf alle $50 \mathrm{~m}$ Hecke ein Goldammer-Revier zu erwarten ist. Bei der Hochrechnung auf Heckenlängen von einem Kilometer entstehen somit unter Umständen unrealistische Dichtewerte, gerade wenn vermehrt Arten auftreten, deren Habitatansprüche nicht auf das Strukturinventar der Hecke beschränkt sind.

Kurze Hecken haben einen großen Randeffekt: Vogelarten, die in ihren Habitatansprüchen nicht allein auf die Sträucher und Bäume einer Hecke als Lebensraum, sondern auch auf die umgebende Landschaft angewiesen sind, können schon sehr kurze Heckenabschnitte besiedeln und erzeugen bei einer Extrapolation der Brutpaardichten hohe Werte für relativ kurze Hecken (vgl. Diskussion Abschnitt Siedlungsdichte).

Die Zunahme der Artenzahl mit der Länge folgt der Beziehung einer Arten-Areal Verlaufskurve, die im Idealfall einer Sättigungskurve entspricht (MACARTHUR \& WILSON 1967, BEGON et al. 1998). In Abb. 25 ist das nicht gegeben, weil erstens die Länge allein nicht ausreichend genau die Fläche einer Hecke abbildet und zweitens die Stichprobe der untersuchten Längenspektren mit 85 \% der Hecken zwischen 100 bis $300 \mathrm{~m}$ zu begrenzt ist. Der in Abb. 25 dargestellte Zusammenhang bezieht sich nur auf einen kleinen Ausschnitt möglicher Heckenlängen. Die Zunahme der Artenzahl mit der Länge der Hecke ist unter Einbeziehung längerer Hecken nicht als linear proportional sondern asymptotisch anzunehmen.

Neben den Volumen bestimmenden Variablen Länge, Höhe und Breite waren für die Brutvogeldichte mit der Abundanz von Hecken und der Dichte des Wegenetzes zwei Landschaftsparameter von Bedeutung. OSBORNE (1984) fand einen positiven Zusammenhang zwischen der Artenvielfalt in Hecken und der Ausbildung von flächigen Gebüschbereichen in 
der Umgebung (Moles \& BreEn 1995, O'Connor \& SHrubB 1986). Die höhere Siedlungsdichte bei höherer Heckendichte in der Landschaft könnte auch im Untersuchungsgebiet auf eine größere Artenvielfalt zurückzuführen sein. In jedem Fall unterstreicht der Faktor 'Heckenabundanz' die Bedeutung von Heckenlandschaften.

Mit zunehmender Wegedichte in der Landschaft nimmt die Siedlungsdichte in den Hecken ab (Tab. 25 und Abb. 24). In der mir bekannten Literatur gibt es bislang keinen derartigen Befund. Als Ursache für den Zusammenhang wären vermehrte Störungen an den Brutplätzen durch landwirtschaftlichen Verkehr oder Freizeitaktivitäten oder ein erhöhtes Prädationsrisiko anzuführen (s.u.). Des Weiteren erfolgt an Wegen in der Regel eine konsequentere Heckenpflege, zumindest was den Rückschnitt von Randbereichen (sogenanntes 'Abschlägeln') und das Ausmähen der Krautschicht entlang aller Feldwege anbetrifft. Im Untersuchungsgebiet wurde jeder wegbegleitende Krautsaum spätestens im Juli gemäht.

In der PCA (Tab. 26) wird ein Faktor maßgeblich von der Variablen 'Krautsaum' dominiert, für die aber weder in der MR noch bei der Kanonischen Korrelation ein Einfluss auf Brutvogelbestände nachzuweisen war. Das bedeutet, dass es durchaus Unterschiede in der Ausdehnung der Säume an den Hecken gibt, dass diese aber auf die Dichte der Brutvogelbestände keine nachweisbaren Auswirkungen zeigen. Das mag an der Uniformität der Säume liegen, deren Artenarmut bezüglich der Pflanzen und Gliedertiere, an der Mahd oder dem Zeitpunkt der Mahd oder andere Gründe haben. Krautsäume könnten theoretisch auch unabhängig von ihrer Breite immer in ähnlicher Weise ökologische Funktionen erfüllen, wie Nistplatz- und Nahrungsangebot sowie Deckungsmöglichkeit bieten. Vor dem Hintergrund der Habitatansprüche vieler Arten und der Kenntnis des Managements dieses Teillebensraums ist das allerdings sehr unwahrscheinlich.

\subsection{Gestaltung von Hecken}

Auf der Ebene von Arten fanden GREEN et al. (1994) aus einem Spektrum von 18 Arten neun, deren Bestand mit der Breite von Hecken korreliert war, darunter Mönchsgrasmücke und Amsel, sowie drei Arten, die negativ mit der Anzahl von Bäumen korreliert war, darunter Bluthänfling und Dorngrasmücke. Der Befund stimmt sehr gut mit den Ergebnissen der Kanonischen Korrelation dieser Studie überein (Tab. 28). In einer Arbeit von MACDONALD \& JOHNSON (1995) korreliert die Dichte von Bluthänflingen negativ, die von fünf anderen Arten dagegen positiv mit der Höhe von Hecken. GREEN et al. (1994) stellten fest, dass zwar die meisten Arten hohe und baumreiche Hecken bevorzugen, dass aber mit Dorngrasmücke (s.a. HINSLEY et al. 1995), Goldammer und Bluthänfling gerade sehr auf Hecken angewiesene 
Arten bevorzugt in kurzen Hecken mit wenigen Bäumen vorkommen. Der auffälligste Zusammenhang zwischen Heckenstrukturen und der Besiedlungsdichte durch Vögel ist der, dass in Hecken mit großer Gehölzausdehnung mehr Arten und diese in höherer Dichte vorkommen. Neben den Variablen 'Breite' und 'Überhälter' erreichen auch die Prädiktorvariablen 'Heckendichte', 'Heckenabundanz' und 'Heckenverbund' hohe Rootwerte. Diese Variablen beschreiben eher das Gehölzvolumen in der Landschaft. Je weniger Lücken in den Hecken, je höher die Anzahl von Hecken und je kürzer die Entfernung zwischen den Hecken ist, desto eher kommen Amsel, Zilpzalp, Mönchsgrasmücke, Gartengrasmücke, Singdrossel, Klappergrasmücke, Buchfink und Heckenbraunelle in den Hecken vor.

Der Befund, dass Hecken mit großer Gehölzausdehnung viele Arten oder hohe Dichten von Vögeln beherbergen, findet sich in (zumindest auf einen der hier dargestellten Parameter reduziert) nahezu allen relevanten Arbeiten zu diesem Thema (neben den bereits genannten auch SHALWAY 1985, LACK 1987, LAKHANI 1994, PARISH et al. 1995, SPARKS et al. 1996). Es liegt also nahe, zu Gunsten von Artenvielfalt und hoher Siedlungsdichte ein entsprechendes Management von Hecken zu fordern und zu fördern. Ziel eines solchen flächendeckenden Planes könnte es sein, vor allem breite und baumreiche Hecken zu gestalten.

Viele Feldvögel oder Farmland-Spezialisten (SIRIWARDENA et al. 2000) sind aber in besonderer Weise an halboffene Habitate mit spärlichem Gehölzbewuchs angepasst. Eine Zunahme von Baumzahl und Heckenhöhe lässt erwarten, dass viele heckentypische Arten in ihrem Bestand abnehmen werden (s.o.: Dorngrasmücke und Bluthänfling, GREEN et al. 1994, MacDonald \& Johnson 1995). Die Ergebnisse dieser Studien zeigen, dass die Bewertungsgrundlagen zum Management von Hecken einer Differenzierung bedürfen. Die Tatsache, dass mindestens 10 Arten als typische Heckenbewohner gelten, zeigt die Bedeutung dieses Habitats für diese Arten (neben den oben genannten z.B. Feldsperling, Elster, Neuntöter, Turteltaube, Rebhuhn). Die meisten der anderen Arten sind ursprünglich Waldarten, die nur gelegentlich an besonders geeigneten, d.h. hohen und breiten Hecken auftreten. Unter diesem Gesichtspunkt sollten Hecken für jene Arten gestaltet werden, die auf diesen Lebensraum angewiesen sind und nicht für Arten, die in den umliegenden Wäldern zum Teil große Populationen bilden.

\subsection{Wie viele Vogelarten muss eine aus ornithologischer Sicht 'gute' Hecke mindestens haben?}

Für den Schutz von Arten, die selten, in ihren Bestandszahlen rückläufig und / oder gefährdet sind und die in heckenreichen Landschaften vorkommen (z.B. Neuntöter, Dorngrasmücke, 
Feldsperling, Bluthänfling, Goldammer, Feldschwirl, Baumpieper, dazu Wachtel und Rebhuhn) ist es nach Befunden dieser Arbeit (Tab. 23) nicht begründbar, die Etablierung von Hecken zu fordern, die darauf angelegt sind, einem Maximum von Arten Lebensraum zu bieten. Diese Arten sind an minimale Ausstattungen der Gehölzstrukturen in Hecken angepasst. Die in solchen Hecken brütenden Vögel könnte man somit als Hecken-Minimalisten bezeichnen. Das schließt nicht aus, dass diese Arten auch in artenreicheren Hecken siedeln können und dort zum Teil höhere Abundanzen erreichen (vgl. Tab. 20). Weitaus wichtiger für die Abundanz und den Bruterfolg von Neuntöter, Goldammer und Bluthänfling ist die Art und Intensität der Bewirtschaft umliegender Flächen (BEZZEL 1982, JAKOBER \& STAUBER 1987, LiLle 1996), auch wenn EyBERT et al. (1995, zit in GLUTZ VON BLOTZHEIM \& BAUER 1997) nach der „Reduktion der Hecken auf die Hälfte und Rückgang des Wiesen- und Brachlandanteils zugunsten von Geteideäckern im Umfeld einer von Hänflingen besiedelten Stechginsterheide” keinen „statistisch signifikanten Einfluss auf den Bruterfolg” feststellen konnten. Mit der Nutzungsänderung wurde der Prädationsdruck auf Nester geringer, der Verlust durch Witterung nahm nach Vermutung der Autoren aufgrund längerer Nahrungsbeschaffungsflüge jedoch zu.

Die Mehrzahl der gefährdeten oder rückläufigen Arten kommt bereits in Hecken vor, die jeweils nur wenige Arten beherbergen (Tab. 23). Insofern lässt sich aus den Befunden dieser Arbeit ableiten, dass es sinnvoll ist, eine hohe Anzahl von Hecken in der Landschaft zu fördern und nicht in erster Linie das Gehölzvolumen einzelner Hecken. Ungeachtet der insgesamt festzustellenden Artenzahl, stellen bereits kleinste Hecken wichtigen Siedlungsraum für gefährdete oder rückläufige Arten dar. Vielfach beruht der aktuelle Gefährdungsgrad dieser Arten auf der Rodung von Hecken und der Beseitigung anderer nicht intensiv genutzter Strukturen in der Landschaft, z.B. Streuobstwiesen, Feldraine, Einzelbäume u.a. (vgl. BEZZEL 1982, TUCKer \& HeAth 1994, WitT et al. 1996, Jedicke 1997, BAUER \& BERTHOLD 2000).

Artenvielfalt und hohe Dichten von häufigen Arten sollten für den Lebensraum Hecke nicht das Kriterium sein, das aus ornithologischer Sicht zwischen ökologisch guten und schlechten Hecken trennt. Es ist vielmehr von Bedeutung, dass die in den Hecken siedelnden Arten in der Lage sind, überlebensfähige Populationen zu etablieren. Dabei ist weniger die einzelne Hecke, als vielmehr die Betrachtung der Gestaltung und Gliederung einer Heckenlandschaft von Bedeutung.

Hecken mit großer Breite, Höhe, vielen und alten Bäumen nähern sich in ihrem Erscheinungsbild und in ihrer Ökologie dem Habitat Wald an. In waldarmen Gegenden kann die Anlage solcher Waldstreifen durchaus sinnvoll sein, z.B. Gehölzinseln (Feldgehölze) und Hecken in 
Bördenlandschaften (OELKE et al. 1992), Waldschutzstreifen als Wind- und Erosionsschutz in Westpolen oder Bulgarien, sog. Shelterbelts (MÜHLEnBERG \& SLOWIK 1997). KujAVA (1994) fand in Großpolen in Shelterbelts, die $4 \%$ der untersuchten Flächen ausmachten, $60 \%$ der Brutplätze aller erfassten Vögel. Linienhaft angelegte Gehölzpflanzungen werden dennoch keinen flächigen Wald in seiner komplexen Funktionalität ersetzen können. Auch 'waldartig' angelegte Hecken werden für Waldvogelarten immer nur Lebensräume zweiter Klasse darstellen. Zum können sich Arten mit spezifischen Waldhabitatansprüchen (z.B. dichtes, flächiges Unterholz oder starkes, stehendes Totholz) nicht in Hecken ansiedeln (z.B. Waldlaubsänger, Schwarzstorch, Weißrückenspecht) und zum anderen haben die Waldarten, die in Hecken siedeln, vielfach geringeren Bruterfolg (z.B. GRAJETZKY 1993, BAIRLEIN \& SONNTAG 1994, BARKOW et al. 2001).

Hecken sind sowohl in ihrem Erscheinungsbild und in ihrer Entstehung, als auch in ihrer Funktion und in ihrer Artzusammensetzung ein eigener Habitattyp (BLAB 1993). Bewohner halboffener Landschaften, wie z.B. das Rebhuhn benötigen Hecken als Schutz, Deckung, Nahrungsort oder zur Jungenaufzucht (s.o.), meiden gleichzeitig aber Waldränder oder haben in deren Nähe geringeren Bruterfolg (PANEK 1994).

Das zeigt, dass es für einen gezielten Artenschutz unerlässlich bleiben wird, von der Autökologie einzelner Arten auszugehen. MüHLEnBERG \& HovestadT (1992) schlagen dazu die tierökologische Bewertung von Habitaten durch Zielarten vor (s.a. HOVESTADT et al. 1991, MüHLEnBerg 1993, Vogel et al. 1996). Dieses Vorgehen ist in Bezug auf Hecken in jedem Fall der Forderung nach „Erhaltung möglichst vollständiger Vogelgemeinschaften” (JEDICKE 1997) vorzuziehen, weil erstens keine Definition einer 'vollständigen' Vogelgemeinschaft zu erreichen ist (z.B. FLADE 1994) und zweitens keine Zielvorgabe einer Dimensionsebene (eine Hecke oder 10 Hecken oder gesamter Landkreis?) vorliegt. Im Sinne eines effektiven Biotopund Landschaftsschutzes ist es Erfolg versprechend, mit der konsequenten Umsetzung der Forschungsergebnisse zur Autökologie von Arten oder der Vorgehensweise nach dem Zielarten-Konzept (z.B. MüHLEnBerg 1993) zu folgen. Bei diesem Ansatz steht nicht die Artenvielfalt eines Lebensraums, sondern die Überlebensfähigkeit der Population einer Art im Vordergrund. Ein erfolgreiches Beispiel mit konkreten Handlungsempfehlungen zum Schutz der behandelten Art liegt beispielsweise für den Raubwürger vor (ROTHAUPT 1997).

\subsection{Bruterfolg und Populationsentwicklung}

Nur 30 \% der kontrollierten Nester hatten Bruterfolg (Tab. 29). Dieser Befund spricht dafür, dass Hecken als Bruthabitat ein sehr großes Risiko bergen, weil viele Nester nicht erfolgreich 
sind. Theoretisch muss jedes Brutpaar mehr als drei Nester mit vollem Gelege anlegen, um einmal erfolgreich zu sein.

Innerhalb der untersuchten Arten treten aber erhebliche Unterschiede auf (Tab. 36). Der Anteil erfolgreicher Nester reicht von $14 \%$ bei der Amsel bis zu $70 \%$ beim Zilpzalp. Es stellt sich die Frage nach der Beurteilung so unterschiedlicher Befunde. Zum einen bietet sich der Vergleich mit anderen Studien zu den betreffenden Arten an und zweitens kann über die Berechnung der Populationsentwicklung unter bestimmten Annahmen kalkuliert werden, ob eine Population mit dem gefundenen Bruterfolg kleiner wird oder wachsen kann.

BAIRLEIN (1978) untersuchte eine Brutpopulation von Mönchsgrasmücken, deren Reproduktionsrate das Überleben der Population aufgrund eines Ausfliegeerfolgs von 2,32 Jungvögeln pro Nest theoretisch ohne Zuwanderung ermöglicht. Dieser Source-Situation stehen die 1,68 Jungvögel pro Nest aus dieser Studie gegenüber, die ohne die Einbeziehung von Migration zu einer Verkleinerung der Population um 21-28\% (Tab. 35) führen. Der Nesterfolg von Mönchsgrasmücken in Hecken ist mit $36 \%$ viel geringer als der von BAIRLEIN et al. (1980) aus 546 Nestkarten errechnete Nesterfolg von 51,9\% oder der über 20 Jahre gemittelte Nesterfolg von 53,2\% aus 1573 Nestern, die zwischen 1981-2000 in Waldstücken in Süddeutschland durch die Vogelwarte Radolfzell kontrolliert wurden (SCHAEFER in prep.). Alle vier untersuchten Grasmückenarten hatten nach Nestkartenmaterial von BAIRLEIN et al. (1980) höheren Nesterfolg als in den untersuchten Hecken: Gartengrasmücke 53,5 \%, Klappergrasmücke 56,7\% und Dorngrasmücke $63 \%$. Übereinstimmend hatte die Dorngrasmücke sowohl nach Auswertung der Nestkarten als auch nach den Befunden dieser Studie den höchsten Nesterfolg der Grasmücken. Dieser Vergleich zeigt deutlich, dass für eine Vielzahl von Arten der Bruterfolg in Hecken geringer ist, als in Waldhabitaten. Da die gleichen Arten betrachtet wurden und sich sogar die Untersuchungszeiträume überschneiden (SCHAEFER in prep.) liegt der Schluss nahe, dass der niedrigere Bruterfolg in Hecken habitatbedingt ist. Die Frage ist, wie hoch der Bruterfolg für eine Art sein muss, damit die Population auch ohne Zuwanderung nicht kleiner wird.

PULLIAM (1988) geht davon aus, dass Arten Lebensräume verschiedener Qualität besiedeln und unterscheidet zwei Habitatqualitäten: Habitate in denen der Reproduktionserfolg einer Art deren Mortalität nicht ausgleichen kann (Sink) und Sources, in denen die Art Populationsüberschüsse erzeugt. Der von WATKINSON \& SUTHERLAND (1995) eingeführte dritte Typus unterscheidet zusätzlich sogenannte Pseudo-Sinks. In Pseudo-Sinks entsteht der Eindruck eines Sink-Habitats, weil mehr Individuen in das Habitat einwandern und die Populationsdichte über der Kapazitätsgrenze liegt. Als Folge brüten weniger Individuen und der Fort- 
pflanzungserfolg wird geringer, als zur Stabilisierung der hohen Populationsdichte nötig wäre. Die hohe Dichte erzeugt niedrigeren Bruterfolg und höhere Sterblichkeit und es entsteht der Eindruck, als sei die Population nicht ohne Immigration überlebensfähig. Pseudo-Sinks sind nur schwer als solche zu erkennen (WATKINSON \& SUTHERLAND 1995), so dass ich mich im Rahmen dieser Arbeit auf die Unterscheidung von Sources und Sinks beschränkt habe.

Nach DiAs (1996) besetzen dominante Individuen Reviere in den qualitativ besten Habitaten, darin werden die höchsten Reproduktionsraten erzielt und Populationsüberschüsse erzeugt. Wenn in diesen Source-Habitaten ein Dichtelimit erreicht ist, müssen unterlegene Individuen in Habitate schlechterer Qualität abwandern. An diese Habitate sind die Individuen in der Regel nicht optimal angepasst, so dass die Produktivität in solchen Gebieten nicht ausreicht, um natürliche Populationsverluste auszugleichen. In so beschriebenen Sink-Habitaten können Populationen nur deshalb langfristig existieren, weil immer wieder Zuwanderung aus SourceHabitaten stattfindet (vgl. auch PulliaM \& DuNNING 1994).

Bislang wurde die Benennung von Sink- und Source-Habitaten anhand von Populationsstudien an einzelnen Arten in verschiedenen Lebensräumen vorgenommen. Bei Populationsstudien an Vögeln wurden vorzugsweise Höhlenbrüter untersucht (ROBERTSON 1972, Krebs 1982, BeshKareV et al. 1994, Dias et al. 1994, Ridington \& GosLer 1995, DiAS \& BLONDEL 1996).

Die Ergebnisse dieser Studie zeigen, dass in Hecken Bedingungen gegeben sind, die sich auf frei brütende Arten entweder als Source-oder als Sink-Habitat auswirken können.

MøLLER (1995) konnte an Amseln feststellen, dass in einer fragmentierten Kulturlandschaft die 'Patchgröße', die flächige Ausdehnung von besiedelten Waldfragmenten, eine Habitatqualität darstellt. Die in kleinen Patches erbrüteten Amseln waren körperlich schwächer und hatten höhere Mortalitätsraten als Jungvögel aus großen Waldstücken. Das führte zu einem geringeren Recruitment in den kleinen Waldpatches aus dem dort erzeugten Nachwuchs, so dass kleine Waldinseln für Amseln Sink-Habitate darstellten.

In Hecken lässt sich die Sink-Situation der Amsel anhand des Bruterfolgs feststellen. Die Anzahl ausfliegender Jungvögel reicht nicht aus, um die Population mittelfristig zu erhalten (Tab. 35). Schlechtere Kondition oder Fitness der Jungvögel, die aufgrund ungünstigerer Habitatbedingungen anzunehmen sind, konnte in dieser Studie nicht hinreichend untersucht werden, weil der Bruterfolg in Hecken insgesamt zu niedrig war. Ausgehend von MøLLER (1995) ist zu vermuten, dass die aus Heckenhabitaten ausgeflogenen Jungvögel nochmals höhere Verluste bis zum Erreichen der Geschlechtsreife erleiden, als Individuen aus Wald- 
habitaten. Insofern ist die Übernahme von Mortalitätsraten aus der Literatur eine Basis, die Überlebensraten von Jungamseln aus Hecken eher zu hoch als zu niedrig ansetzt.

Auch der Bruterfolg der Singdrossel ist in Hecken zu gering, um die natürliche Mortalität aufzufangen. Gegenüber Arten wie Zilpzalp und Heckenbraunelle, für die Hecken SourceHabitate darstellen, unterschieden sich die Drosselarten in weiteren brutbiologischen Parametern, zum Beispiel in der Gelegegröße. Heckenbraunelle und Zilpzalp legten durchschnittlich 1,3 Eier je Vollgelege mehr, so dass erfolgreiche Bruten mehr Jungvögel und damit einen höheren Ausfliegeerfolg bedeuteten. Bei der Heckenbraunelle wird dieser jedoch durch einen sehr hohen Anteil von $27 \%$ unbefruchteter Eier vermindert. Ein hoher Anteil unbefruchteter Eier ist für diese Art aber auch aus anderen Habitaten dokumentiert (vgl. BIRKHEAD 1981, SNOW \& SNOW 1982) und nicht auf verminderte Habitatqualität zurückzuführen. Der Schlupferfolg der Heckenbraunelle ist daher noch geringer als der von Amsel und Singdrossel (Tab. 30).

Beim Zilpzalp schlüpften zwei Drittel aller in Vollgelegen erfassten Eier, die Anzahl unbefruchteter Eier war sehr gering und aufgrund geringer Totalverluste war der Nesterfolg mit $70 \%$ von allen untersuchten Arten am höchsten. Bei der Heckenbraunelle ist zwar nur jedes fünfte Nest erfolgreich, da aber die Mortalität der Altvögel nach einer von SNOW \& SNOW (1982) in UK durchgeführten Studie nur bei etwa $30 \%$ liegt (Tab. 34), reicht ein Ausfliegeerfolg von etwa 0,8 Jungvögeln auf alle angefangenen Nester zum Erhalt einer lokalen Population, wenn die Mortalität der Jungvögel die angenommene Rate von 0,5 nicht zu stark übersteigt (Literaturdaten zur Sterblichkeit von Heckenbraunelle und Zilpzalp im ersten Lebensjahr waren nicht verfügbar). Amsel und Singdrossel konnten die allgemeine Sterblichkeit der Alt- und Jungvögel (Literaturwerte, Tab. 34) nicht kompensieren. Um einen Jungvogel hervorzubringen müssen beide Arten jeweils zwei Gelege anfangen. Das bedeutet, dass ein Singdrosselpaar sieben Vollgelege anlegen muss, um ein Nest mit vier Jungvögeln zum Ausfliegen zu bringen. Bei der Amsel reichen nicht einmal sieben Brutversuche aus. Ein Zilpzalp erzeugt mit zwei Brutversuchen mehr als fünf Jungvögel. Bei diesem Vergleich wird deutlich, dass der Lebensraum Hecke für Singdrossel und Amsel ein Sink-Habitat ist, für Zilpzalp und Heckenbraunelle (bei Gültigkeit der geringen Mortalität von Altvögeln der Art) sind die Hecken um Göttingen ein Source-Habitat.

Goldammer und Dorngrasmücke sind in ihrer Habitatwahl stark an Hecken gebunden und erzielen dort Bruterfolg, der das Fortbestehen ihrer Populationen ermöglicht. Bei den anderen drei Grasmückenarten ist das in diesem Lebensraum nicht der Fall (Tab. 30). Dabei sind die Gelege dieser Arten größer, und die Gartengrasmücke hat einen besseren Schlupferfolg als 
die Dorngrasmücke. Letztendlich ist aber der Nesterfolg bei der Dorngrasmücke mit $50 \%$ deutlich höher als bei den anderen Grasmücken (29-36\%). Hier wird die schlechtere Anpassung der fakultativ in Hecken siedelnden Arten deutlich, denn die Mehrzahl der Nester von Mönchs-, Garten- und Klappergrasmücke hat keinen Bruterfolg.

Die Anzahl der Reviere von Mönchs- und Gartengrasmücke zeigten in den drei Untersuchungsjahren nur geringe Veränderungen (Tab. 21). Nach den Bruterfolgen zu schließen, müssten beide Populationen kontinuierlich abnehmen. Da das nicht in allen Jahren der Fall ist, können die Populationszuwächse nur auf der Zuwanderung von Individuen aus anderen Gebieten zurückzuführen sein. Der Befund für die Klappergrasmücke ist aufgrund der geringen Anzahl kontrollierter Nester nicht gesichert. Die Art hatte sehr sprunghafte Bestandsveränderungen (Tab. 21), vor allem von 1999 auf 2000, als sich der Brutbestand in den Hecken mehr als verdoppelte. Auch hier ist anzunehmen, dass die Mehrzahl der Reviere in den Hecken von Zuwanderern besetzt worden ist.

\subsection{Warum können Hecken für manche Arten ein Sink- und für andere Arten ein Source-Habitat sein?}

Die Entscheidung, ob ein Habitat zu einem sink oder source für eine Art wird, fällt anhand des Neststandortes, der eng mit dem Prädationsrisiko verknüpft ist und der Flexibilität der Art, auf veränderte Strukturbedingungen in den Hecken zu reagieren. Hecken erfordern für offen brütende Singvogelarten somit besondere Anpassungen an diesen anthropogen geformten Lebensraum.

Die Nester erfolgreich brütender Arten waren tendenziell niedriger angelegt. Zilpzalpe brüten bodennah (GLUTZ vON BLOTZHEIM 1991b). Die Dorngrasmücke hat von allen vier vorkommenden Grasmücken die niedrigste Nesthöhe (BAIRLEIN et al. 1980) und ist die einzige Grasmückenart, für die aufgrund des Bruterfolgs in Hecken eine stabile Populationsentwicklung ohne Zuwanderung prognostizierbar ist (Tab. 35). Amsel und Singdrossel brüten von den untersuchten Arten am höchsten.

Späte Nester waren öfter erfolgreich als frühe. Dieses Ergebnis steht in Übereinstimmung mit BAIRLEIN (1978), der an den Erstbruten von Mönchsgrasmücke einen Nesterfolg von 52 \% und an den Nachgelegen und Zweitbruten 75-80\% feststellen konnte. Die später in der Brutperiode angelegten Nester der Goldammer waren aber höher angelegt als die weniger erfolgreichen Nester der Erstburt im Mai (Abb. 26). Das steht scheinbar im Widerspruch zu der Korrelation zwischen erfolgreichen Arten und der durchschnittlichen artspezifischen Nesthöhe. In Anpassung an die Vegetationsentwicklung kann die Goldammer flexibel auf 
veränderte Habitatbedingungen reagieren. Die Goldammer nutzt die Vegetation im Krautsaum oder Heckenmantel erst, wenn diese dicht genug ist, um Nester wirkungsvoll zu verstecken. Die Heckenbraunelle zeigt ein ähnliches Verhalten, denn viele Nester, die im April angelegt wurden, sind in den noch kargen Hecken in Totholz, auf vorjährigen Brennesseln oder um das Stammholz in noch nicht ausgeschlagenen Schlehen angelegt. Spätere Gelege finden sich eher in dichtem Lonicera- oder Brombeer-Gebüsch, das mit Brennessel und Klettenlabkraut durchwachsen ist (Zusammenfassung der Neststandorte von 19 Nestkarten dieser Arbeit). Bei der Wahl der Neststandorte sind Amsel und Singdrossel in Hecken sehr eingeschränkt. Die umfangreichen Nester brauchen tragfähige Astverzweigungen als Unterlage. In Hecken kommen somit für diese Arten nur wenige potentielle Nestplätze in Betracht. Die Möglichkeit, das Nest im Verlauf der Brutsaison besser versteckt anlegen zu können, ändert sich durch geringe Belaubung an den starken Ästen im Innenbereich der Hecken nur geringfügig.

Die Beispiele zeigen, dass eine geringe Flexibilität in der Anlage und Wahl der Neststandorte in Abhängigkeit fortschreitender Vegetationsentwicklung der Grund dafür sein kann, dass Hecken für manche Arten ein Sink-Habitat darstellen.

Für Amsel, Singdrossel, Mönchs-, Garten- und Klappergrasmücke stellen Hecken zwar ein Sink-Habitat dar, aber keine der genannten Arten blieb in Hecken ohne Bruterfolg. Bei der geringen Lebenserwartung der meisten Singvögel mit Mortalitätsraten zwischen 30-70 \% (vgl. Literatur in Tab. 34) müssen die Arten jede Brutmöglichkeit nutzen, sofern eine Chance auf Bruterfolg besteht. Des Weiteren besteht ein Druck in Richtung Erstbrut, denn die Jungvögel erfolgreicher Erstbruten haben die besten Chancen als Recruits im nächsten Jahr erfolgreich zu brüten, weil sie in der Regel die Jungvögel mit der besten Fitness und Erfahrung werden und somit bessere Überlebenschancen bis zur nächsten Brutzeit haben (Tinbergen 1990, Møller 1994, Brown \& Brown 1999).

Für Goldammer, Dorngrasmücke, Heckenbraunelle und Zilpzalp stellen Hecken ein SourceHabitat dar. Für den Zilpzalp lagen nur wenig Nestfunde vor, so dass die Einschätzung auf einer sehr schlechten Datengrundlage erfolgte. Der berechnete Populationsüberschuss dieser Art zeigte auch die stärksten Abweichungen bei unterschiedlicher Methodik zur Berechnung der relativen Populationsgröße im Folgejahr (Tab. 35). Bei der Heckenbraunelle ist der Status sehr stark von der geringen Mortalität von Altvögeln aus der Studie von SNOW \& SNOW (1982) abhängig. Mit Dorngrasmücke und Goldammer haben zwei Arten in Hecken ein Source, was man auch erwarten sollte, da beide Arten jeweils mit einem großen Anteil ihrer 
Population in diesem Lebensraum siedeln und zumindest über die letzten drei Jahrzehnte keine flächenhaften Abnahmen in Deutschland zu verzeichnen hatten.

\subsection{Ursachen für Brutverluste}

Etwa $70 \%$ der in dieser Studie kontrollierten Nester hatten keinen Bruterfolg und bei $54 \%$ war Prädation die Ursache (Tab. 29). Dazu kamen weitere Verluste von 10 bis $20 \%$ aus anderen Gründen, die vor allem auf anthropogene Aktivitäten zurückzuführen waren. Über die drei Untersuchungsjahre betrachtet war die Prozentverteilung ausgeraubter Nester sehr ausgeglichen, während der Anteil 'sonstiger Verluste von 1999 auf 2000 um über 50 \% abnahm (Tab. 29). Anthropogen und durch Prädation bedingte Gelegeverluste führen in ihrem Zusammenwirken dazu, dass Hecken für viele Vogelarten Sink-Habitate darstellen.

Nestverluste durch Prädation können bei offen brütenden Arten auch in anderen Habitaten sehr hoch sein. WILSON \& COOPER (1998) fanden an über 500 Nestern des offen brütenden Acadian Flycatcher ('Buchentyrann', Empidonax virescens) $52 \%$ der Nester in einem Tieflandwald ausgeraubt. Eine Prädationsrate von $54 \%$ stellt somit nicht zwangsläufig ein spezifisches Problem für Brutvögel in Heckenstrukturen dar.

Der Vergleich der Bruterfolge von Grasmücke in dieser Studie mit den Ergebnissen von BAIRLEIN (1978), BAIRLEIN et al (1980) und SchAEFER (in prep.) zeigte jedoch, dass der Nesterfolg in Hecken habitatbedingt erheblich geringer ist als in Waldhabitaten (12.8). Der augenscheinlichste Unterschied zwischen Hecken und Waldhabitaten ist die Linearität der Hecken. Diese ermöglicht es den potentiellen Nesträubern effektiv Nester zu finden. Zudem ist die Revierdichte in Hecken enorm hoch, so dass auch die Nesterdichte erheblich höher ist als in flächigen Waldhabitaten. Weil auch der Prädationsdruck selbst dichteabhängig ist (MARTIN 1988, RiCKLEFS 1989), wäre zu erwarten, dass Verluste durch Prädation in Hecken mit hoher Siedlungsdichte größer sind als in Hecken mit geringer Siedlungsdichte. Somit könnte hoher Bruterfolg durch hohe Siedlungsdichte in Verbindung mit erhöhter Prädation vermindert werden.

Bei der Goldammer werden 46,6 \%, bei der Dorngrasmücke nur 26,7 \% der Nester ausgeraubt. Beide Arten gelten als Charaktervögel von Heckenlandschaften und erreichen darin sehr hohe Siedlungsdichten (Tab. 20). Vor allem bei der Dorngrasmücke sind die Verluste durch Prädation so gering, dass der Bruterfolg ausreichen sollte, um eine ohne Zuwanderung überlebensfähige Population zu erhalten. Die Dorngrasmücke verliert in Hecken jedoch nochmals fast ein Viertel aller Vollgelege durch anthropogen bedingte Verlustursachen (Tab. 36). Damit stellen Verluste durch Mahd und Störungen eine quantitativ bedeutsame Verlust- 
ursache für diese Art dar, die dadurch in Hecken kaum mehr Nachwuchs erzeugen kann, als zum Erhalt des aktuellen Populationsniveaus unbedingt notwendig ist. Höhere Mortalität auf dem Zug oder im Überwinterungsquartier, wie bei der Art schon passiert (BERTHOLD 1973), könnte unter den gegebenen Bedingungen kaum oder nur über lange Zeiträume aufgefangen werden.

Bei der Goldammer können wenige zusätzliche Gelegeverluste zu spürbaren Auswirkungen auf die Population führen, weil die Art über $45 \%$ prädationsbedingte Gelegeverluste hat. Die erfolgreicheren Nester sind die, die ab Juni angelegt werden (vgl. Kap. 11.6), so dass das Ausmähen der Säume von Hecken im Juni vor allem Nester betrifft, die mit erhöhter Wahrscheinlichkeit nicht ausgeraubt worden wären.

Prädationsverluste waren insbesondere zu Beginn der Brutzeit hoch (Abb. 27). Früh im Jahr brütende Arten wie Amsel, Singdrossel und Heckenbraunelle verloren im April etwa $80 \%$ ihrer Nester durch Prädation. Zusammen mit anderen Verlustursachen kann es für diese Arten in bestimmten Jahren zu Totalausfällen der Erstbrut kommen. Für eine Vielzahl von Hecken dürfte das zutreffen, der Beweis über die Kontrolle aller Nester wäre jedoch noch zu erbringen.

Auch Arten, die im Untersuchungsgebiet erst im Mai ihre Erstbrut anlegen, haben noch sehr hohe Verluste durch Prädation. So ist der Nesterfolg der Erstbrut bei der Goldammer nur halb so hoch, wie der späterer Gelege. Das stimmt mit den Befunden von LILlE (1996) überein, der ebenfalls einen signifikant besseren Bruterfolg der Goldammer in der zweiten Brutphase fand. BAIRLEIN (1978) stellte bei Mönchsgrasmücken signifikant höheren Nesterfolg an Folgegelegen fest.

Nach den Ergebnissen der Studie mit Kunstnestern waren die Nestverluste Ende Mai am geringsten (Tab. 40), während an den echten Nestern erst Mitte Juni die geringsten Prädationsverluste (Abb. 27) auftraten. Im Juni und Juli raubten Elstern viel weniger Nester aus, als von April bis Mai, als die Art häufigster Nesträuber an den Fotonestern war (Tab. 39). Beim Eichelhäher war es umgekehrt. Insgesamt fiel dabei der Anteil ausgeraubter Nester von über 70-80 \% im April/Mai auf weniger als $35 \%$ im Juni/Juli. Dieses lässt vermuten, dass nach der Brutzeit in Gruppen durch die Hecken ziehende Eichelhäher geringere Nestverluste als Elstern im April/Mai bewirken. Im April erzeugen vorwiegend Elstern mit 80 \% die höchste Ausraubrate an offenen Singvogelnestern. Die unbelaubten Schlehen erlauben eine gute Durchdringbarkeit der Gebüsche und den brütenden Vögeln bieten sich nur wenige Versteckmöglichkeiten. Im Mai wird die Vegetation dichter, aber die meisten Arten brüten im Mai, so dass sich die Suche nach Gelegen aufgrund einer hohe Nesterdichte lohnt (KEYSER et 
al. 1998). Im Juni wird der Prädationsdruck auf Nester erheblich geringer (Abb. 27). Die Anzahl von Elstern, die an Kunstnestern mit Kamera auftraten, ging im Juni und Juli stark zurück. Es ist wahrscheinlich, dass sich einerseits die Suche nach einer geringeren Anzahl von Gelegen nicht mehr lohnt und dass andererseits das Nahrungsangebot auf umliegenden Nutzflächen besser ist, als in den Hecken. Zudem kann es eine Rolle spielen, dass der Bedarf an Protein, das die Gelege bieten, im April und Mai zur eigenen Produktion der umfangreichen Gelege höher ist und deshalb in diesen Monaten eine gezielte Suche nach Nestern stattfindet, was im Juni entfällt.

Eichelhäher treten erst ab Juni-Juli verstärkt als Nesträuber in den Hecken in Erscheinung. Es scheint sich dabei um in Gruppen herumstreifender Jungvögel zu handeln, die in diesem Zeitraum auch häufig vor allem in waldnahen Hecken beobachtet werden konnten. Anhand des steigenden Bruterfolgs lässt sich schließen, dass Eichelhäher in Hecken viel weniger effizient Nester ausrauben als Elstern das im Frühjahr tun.

Das Bild, dass sich aufgrund der Fotonester ergibt, frühe Nester werden von Elstern, spätere vor allem von Eichelhähern ausgefressen, kann nur als grober Anhaltspunkt für das Prädationsgeschehen an Singvogelnestern herangezogen werden. Wie bei allen Studien mit künstlich hergestellten Nestern lassen sich die Befunde nur unter Vorbehalt interpretieren, weil die Kunstnester nicht von Altvögeln bewacht oder verteidigt werden, die Nester oft weniger gut getarnt und versteckt sind, die Neststandorte nicht identisch sind, anderes Nistmaterial verwendet wird, die Eier größer und anders gefärbt sind oder sogar künstliche (Plastilin-) Eier verwendet werden, die Neststandorte durch Ausbringung und Kontrollen für beobachtende oder olfaktorisch orientierte Räuber leicht auszumachen sind, künstlich hohe Nestdichten erzeugt werden und weil häufig vollkommen unklar ist, welche Räuber die Nester ausfressen und welche Auswirkungen das auf die Prädationsmuster hat (MAJOR \& Kendal 1996). Trotz dieser Fülle von Vorbehalten, machen Studien mit Kunstnestern Sinn, wenn es darum geht, bestimmte Muster $\mathrm{zu}$ erkennen und $\mathrm{zu}$ deuten. Die wichtigste Voraussetzung für eine gute Interpretation der Befunde ist die Kenntnis der wichtigsten Nesträuber und die konnten in dieser Studie ermittelt werden. Mit dem Wissen wer die Kunstnester zu welcher Phase der Brutzeit ausraubt, lassen sich die Ergebnisse der Logistischen Regressionen zu den Neststandorten und den Heckenstandorten besser interpretieren. Wenn wir davon ausgehen, dass die Mehrzahl der Nester von Corviden ausgefressen wurde, dann wird sehr leicht verständlich, dass vor allem schmale Hecken ausgeraubt wurden. Die Nester konnten dort leicht gefunden werden, gerade wenn die Hecken hoch aufgewachsen waren, denn Bäume in den Hecken verhindern häufig einen dichten 
Bewuchs der Strauchschicht und haben zudem durch exponierte Sitzwarten anziehende Wirkung auf die Rabenvögel. Dass auch ein hoher Krautsaum und dichte Vegetation um das Nest zu einer höheren Ausraubrate führte, erscheint auf den ersten Blick zunächst etwas widersinnig. Es ist aber möglich, dass in hohen Krautsäumen eher Spuren von der Ausbringung oder den Kontrollen bleiben und dass gerade Vegetationsverdichtungen im Heckensaum bei einem systematisch suchenden Nesträuber 'kontrolliert' werden.

Es gab in der Analyse keine weitere Variable im Modell, die darauf hinweisen würde, dass besonders gut versteckte Nester auch besonders sichere Nester seien. Weder die Dichte der Hecke, noch die Sichtbarkeit der Nester von oben oder von der Seite hatte einen Einfluss darauf, ob ein Nest ausgeraubt wurde oder nicht. Das spricht für die Theorie von WILSON \& COOPER (1998), die in der Prädation ein zufälliges Ereignis in Raum und Zeit sehen. In dieser Studie konnte ich zeigen, dass es für ein Nest ausschlaggebend war, ob eines der benachbarten Nester ausgeraubt worden ist. Wenn dieses der Fall war, so war die Wahrscheinlichkeit hoch, dass das betrachtete Nest auch ausgeraubt wurde. War keines der Nachbarnester ausgeraubt, so fiel die Ausraub-Wahrscheinlichkeit des betrachteten Nestes unter $10 \%$. Die Kunstnester wurden heckenweise ausgeraubt. In Hecken die nicht ausgeraubt wurden, überlebten auch relativ offene, gut sichtbare und leicht erreichbare Nester. Dieses Ergebnis zeigt, dass das Schicksal eines Nests weniger durch unmittelbare Neststandortfaktoren als vielmehr von Faktoren bestimmt wurde, die im regionalen Landschaftskontext $\mathrm{zu}$ sehen sind. Das wurde im Zusammenhang mit der zweiten Logistischen Regression zur Erfassung des Einflusses der Heckenstrukturen und der Heckenumgebung auf die vollständige Ausraubung aller sechs ausgebrachten Nester je Hecke untersucht. Da die Untersuchung in der zweiten Hälfte im April durchgeführt wurde, kann davon ausgegangen werden, dass die meisten Nester von Elstern ausgeraubt worden sind.

Das Modell integriert drei Variablen, von denen nur eine für sich genommen signifikant ist: Mit steigender Wegedichte in einem 10 ha großen Landschaftsausschnitt um die Hecken, nahm die Prädation zu. Im Untersuchungsgebiet wurden Feldwege von Elstern gegenüber allen anderen Flächentypen bevorzugt zur Nahrungssuche genutzt (JAHN 2000). Es ist naheliegend, dass die ausgebrachten Kunstnester von Elstern gerade bei einem schmalen Gebüschmantel über die Nutzung von Feldwegen zur Nahrungssuche gefunden wurden.

Eine direkte Meidung von Waldrändern bezüglich der Brutplatzwahl konnte im Untersuchungsgebiet (JAHN 2000) nicht nachgewiesen werden. Nach MÄCK \& JÜRGENS (1999) besiedelt die Elster auch 'schmale Waldstreifen oder Waldbestände mit größeren Freiflächen'. Bei der Nahrungssuche auf Feldwegen ist jedoch zu vermuten, dass die Elster unmittelbare 
Waldnähe umgeht, um ein größeres Prädationsrisiko zu vermeiden. Des Weiteren wird die Wegedichte bei zunehmendem Anteil von Waldfläche geringer, so dass zudem weniger Fläche zur Nahrungssuche zur Verfügung steht.

Somit ergibt sich ein schlüssiges Bild, mit dem das Prädationsmuster an Kunstnestern in Hecken erklärt werden kann: Die Kunstnester werden vor allem von zwei Corviden-Arten ausgeraubt, dabei ist nicht entscheidend, wie gut die einzelnen Nester versteckt sind, sondern in welchen Hecken die Nester ausgebracht sind.

Dieses gilt insbesondere in fragmentierten Landschaften, zu denen die halboffene Kulturlandschaft Mitteleuropas zu zählen ist, in der Wald nur noch fragmentarisch erhalten ist (ANDRÉN \& ANGELSTAM 1988, ANDRÉN 1995). Hecken stellen als linienhaft angelegte Gehölzstreifen eine besondere Erscheinungsform von Lebensraum in der Kulturlandschaft dar. Es war zu vermuten, dass der Prädationsdruck in Hecken besonders hoch ist, weil die Linienstruktur für gehölzbrütende Arten nur wenig Möglichkeiten zur Anlage von Nestern bietet. Systematisch suchende Nesträuber sollten gerade in den häufig dicht besiedelten Hecken hohen Sucherfolg haben, der geringe Bruterfolg vieler Heckenbrüter resultiert demnach aus linienhafter Habitatstruktur und hoher Siedlungsdichte.

\subsection{Naturschutz und Forschung in Hecken}

Zur Bewertung von Habitaten im Sinne des Naturschutzes ist es nicht ausreichend, nur das Arteninventar zu vergleichen. Die Verteilung der Arten, die Stetigkeit, Dominanz und Siedlungsdichte sind weitere wichtige Faktoren, die zusätzlich zur genaueren Beschreibung von Brutvogelgemeinschaften herangezogen werden sollten. Anhand dieser Kennwerte lassen sich bereits viele Faktorengefüge beschreiben und Veränderungen dokumentieren. Aber gerade der Vergleich der aktuellen Daten mit den Ergebnissen der Arbeit von DIETRICH (1903) zeigt, welche Möglichkeiten die Langzeitbeobachtung von Tierpopulationen bietet. Verschiebungen von Artgefügen entwickeln sich häufig über längere Zeitabschnitte und gehen allmählich von statten, so dass sie kaum wahrnehmbar sind. Von daher sind langfristig angelegte Monitoring-Programme für den Natur- und Artenschutz so bedeutsam (BAIRLEIN et al. 2000, DRÖSCHMEISTER 2001).

Das Erkennen einer Veränderung in einer Populationen ist aber nur der Anfang von weiteren Handlungsschritten, die darauf folgen sollten, denn vielfach sind mit dem Rückgang von Arten nicht auch gleichzeitig die Ursachen erkennbar. Die Gründe für den Rückgang von vielen Wirbeltierarten sind oftmals nicht vordergründig zu erkennen ( wie z.B. direkte Tötung durch Jagd, Verkehr oder Vergiftung), sondern werden diffus mit Habitat- oder Umweltverän- 
derungen beschrieben. Die Auswirkungen dieser Veränderungen auf die Populationen müssen erkannt werden, um wirksame Gegenmaßnahmen ergreifen zu können. Dazu müssen wir wissen, wie sich die Populationen in den jeweiligen Lebensräumen entwickeln, d.h. wir benötigen Kenntnisse zur Populationsgröße, deren Aufbau und Verteilung (MÜHLENBERG 1982, 1998, BAIRLEIN 2000). Darüber hinaus bedarf es der Kenntniss und der Dokumentation lokaler Einflussgrößen (Eingriffe, Veränderungen, Freisetzung von Tieren, Nutzungswandel).

Als Beispiel sei der Bestand des Rebhuhns in der Heckenlandschaft um Göttingen angeführt. Im Rahmen dieser Untersuchung konnten noch Balz und auch vereinzelt Paare festgestellt werden. Brutnachweise durch Gelege, Küken oder Familien konnten dagegen nicht erbracht werden. Von Jägern wurde mir berichtet, dass in einigen meiner Untersuchungsflächen Rebhühner ausgesetzt worden sind. Somit muss unklar bleiben, ob die Population dieser Art um Göttingen überhaupt aufgrund von natürlichen (Rest-) Beständen existiert oder ob nur aufgrund der Freisetzungen noch vereinzelt Tiere oder Paare $\mathrm{zu}$ beobachten sind. Es ist ungeklärt, ob die verbliebenen Tiere Nachwuchs haben und ob dieser ausreicht, die natürliche Sterblichkeit auszugleichen. Nur in diesem Fall wäre die Population überlebensfähig. Dieses Beispiel zeigt, dass der alleinige Nachweis einer Art noch keine gute Referenz für die ökologische Qualität des betreffenden Gebietes ist. Für den Landkreis Göttingen ist eher der Einschätzung DöRRIES (2000) zu folgen, der davon ausgeht, dass ,dieser frühere Allerweltsvogel [...] aus unserer Region zu verschwinden droht."

Die Reviergröße beim Rebhuhn entspricht der Fläche, die ein Brutpaar überblicken kann. Mit einer starken Kammerung der Landschaft durch Hecken kann die Brutpaardichte dieser Art effektiv erhöht werden (BLAB 1993). Des Weiteren ist das Nahrungsangebot in Frühjahr und Sommer ein entscheidender Faktor für eine erfolgreiche Jungenaufzucht. Die Küken ernähren sich zunächst animalisch und ab Juli-August zunehmend vegetabil (DwENGER 1991). Die hohe Sterblichkeit der Jungvögel wird auf ein schlechtes Nahrungsangebot durch intensive Landnutzung zurückgeführt (RANDS 1985, POTTS 1997). Im Winter werden von Rebhühnern Hecken bevorzugt und vor allem Ackerbrachen und Stoppelfelder, Maisstoppel, Luzerne, Raps und Wiesenbrachen aufgesucht, wohingegen 'nackte' Ackerflächen und Schläge mit Wintergetreide gemieden werden (KAISER 1997). Allein aufgrund dieser wenigen Informationen wird sofort deutlich, warum das Rebhuhn im Landkreis Göttingen keine mittelfristige Überlebenschance hat: Ackerflächen werden sofort nach der Ernte umgebrochen, um Wintergetreide einzusäen, der Eintrag von Pestiziden und Dünger lässt nichts als das Wachstum der Zielpflanzen zu, Randstreifen sind auf ein Minimum an Fläche zurückgedrängt, eine hohe Heckendichte ist nur in wenigen Bereichen erhalten geblieben. Dieses Beispiel zeigt, dass der 
Nachweis der Art nicht nur ein schwaches Indiz für die Habitatqualität des betreffenden Lebensraums ist sondern auch zu einer Fehleinschätzung der Lebensraumqualität führen kann, wenn wie in diesem Fall auch regionale Auswilderungen stattfinden. Die Erfassung der Art durch Kartierungen ist dann, außer zur Erfolgskontrolle der Auswilderungsmaßnahme, bedeutungslos.

Darüber hinaus wird deutlich, dass der Erhalt von Hecken allein die Brutvogelbestände in ihrer Artenvielfalt nicht trägt. Viele Arten sind auf die Nutzbarkeit des Umlandes angewiesen, brüten aber in den Heckenstrukturen. Die hohen Revierdichten in den Hecken um Göttingen mit 880 Brutpaaren auf 10 ha relativieren sich erst, wenn man die Nutzung der umliegenden Flächen mit einrechnet auf 18 BP/10ha.

Wenn von der Intensivierung der Landwirtschaft die Rede ist, wird häufig auf das Verschwinden von Hecken hingewiesen (z.B. TISCHLER 1990). Rodungen von Hecken finden heute nicht mehr im großen Maßstab statt, wie noch in den 1960er und 70er Jahren, vielfach werden sogar neue Hecken in der Agrarlandschaft angelegt. Das war zum Beispiel auch aktuell im Untersuchungsgebiet Volkerode der Fall. Dennoch verzeichnen gerade Vögel der Agrarlandschaften die stärksten Bestandsrückgänge (TUCKER \& HEATH 1994). Die Ursachen liegen in der anhaltend intensiven Nutzung der bewirtschafteten Flächen. Hier gibt es weiterhin Forschungs- und Handlungsbedarf.

Die Einführung von Flächenstilllegungs- und Ackerrandstreifenprogrammen mit der Schaffung von ungenutzten Bracheflächen zeigt positive Auswirkungen auf den Bruterfolg der Goldammer (LILLE 1996) und der Grauammer (FISCHER \& SCHNEIDER 1996). Doch es bedarf weiterer und intensiverer Bemühungen um den Naturschutz in der Fläche, um auch Arten wie das Rebhuhn, den Raubwürger und den Neuntöter wieder in überlebensfähigen Populationen in der Kulturlandschaft Deutschlands beobachten zu können. 


\section{Zusammenfassung}

Aus der Bearbeitung des Heckenprogramms der deutschen Vogelwarten ergaben sich Fragestellungen, die über das Heckenprogramm hinaus weitere Aspekte aufgreifen oder vertiefend behandeln sollten. Diese lassen sich in drei Themenkomplexen zusammenfassen.

1. Welche Beziehungen bestehen zwischen Brutvogelbeständen und Heckenstrukturen unter Einbeziehung der umgebenden Landschaft?

2. Welche brutökologische Bedeutung haben Hecken für Singvögel?

3. Wodurch wird der Bruterfolg in Hecken vermindert und wie wirken sich unterschiedliche Hecken- und Landschaftsstrukturen dabei auf den Prädationsdruck aus?

Zur Durchführung der Freilandstudie wurden um Göttingen fünf Untersuchungsgebiete ausgewählt, in denen zwischen 1998 und 2000 Brutvogelbestände und Heckenstrukturen kartiert wurden. In den 30 bearbeiteten Hecken wurden jedes Jahr Nester offen brütender Singvögel gesucht und der Bruterfolg erfasst. Anhand von Kunstnestern wurden zeitliche und räumliche Prädationsmuster in Hecken beschrieben und mit Neststandorten und Heckenstrukturen in Beziehung gesetzt.

Die untersuchten Hecken waren mit etwa $90 \mathrm{BP} / \mathrm{km}$ sehr dicht besiedelt. Die mitttlere Entfernung zwischen zwei Revieren betrug 11,5 m. Es wurden 41 Brutvogelarten erfasst, die Diversität über alle Hecken lag bei $\mathrm{H}_{\mathrm{s}}=3,1$. Die mit Abstand häufigste Art war die Goldammer, die in jeder Hecke in mindestens einem Jahr brütete. Mit Heckenbraunelle, Amsel, Dorngrasmücke, Feldsperling, Mönchs- und Gartengrasmücke folgten weitere dominante Arten mit abfallender Häufigkeit.

In allen Hecken die jeweils weniger als 15 Arten beherbergten kommen zusammen $65 \%$ aller nachgewiesenen Arten vor. In besonders artenreichen Hecken, mit jeweils über 20 Arten, treten als 'neue' Arten nur noch Zaunkönig, Buntspecht, Rabenkrähe, Kleinspecht und Kernbeißer auf. Unter diesem Aspekt wird die Frage diskutiert, ob Hecken artenreich sein müssen, um einen hohen Naturschutzwert zu haben.

Die Menge und Ausdehnung an Gehölzen in der Hecke und der Landschaft bestimmt im Wesentlichen die Vogelabundanz. Das Ergebnis einer Multiplen Linearen Regression zeigt die Bedeutung der Variablen 'Breite', 'Länge', 'Überhälter' (Maß für die Höhe der Hecke) und die 'Heckenabundanz' (Anzahl der Hecken in der Umgebung). Eine hohe Wegedichte vermindert dagegen die Siedlungsdichte in Hecken. 
Hohe Siedlungsdichten in Hecken mit großen Gehölzvolumen werden vor allem durch hohe Dichte von Waldarten erzeugt. Das Ergebnis einer Kanonischen Korrelation zeigt, dass auf die Variablen 'Breite' und 'Überhälter' mit Amsel, Zilpzalp, Mönchsgrasmücke, Gartengrasmücke, Singdrossel, Klappergrasmücke, Buchfink und Heckenbraunelle vor allem ursprüngliche Bewohner lichter Waldhabitate positiv ansprechen, während die Häufigkeit von Bluthänfling, Dorngrasmücke, Sumpfrohrsänger, Grünling. Goldammer und Rotkehlchen nicht beeinflusst wird.

Die Bedeutung dieser Ergebnisse wird im Hinblick auf Naturschutzziele diskutiert. Es erscheint sinnvoller Hecken zu Gunsten von Arten zu pflegen, die auf diesen Lebensraum in Ermangelung natürlicher Habitate angewiesen sind, auch wenn damit eine geringere Artenzahl und Siedlungsdichte verbunden ist, weil bestimmte Waldarten dann nicht mehr in den Hecken auftreten.

Der Bruterfolg der meisten Arten war außerordentlich gering. Bei weniger als einem Drittel (30,5\%) aller Nester wurden Jungvögel flügge. Den höchsten Nesterfolg hatten Zilpzalp $(70 \%, \mathrm{~N}=10)$, Dorngrasmücke $(50 \%, \mathrm{~N}=34)$ und Goldammer $(42 \%, \mathrm{~N}=45)$. Bei Amsel $(14 \%, \mathrm{~N}=36)$, Singdrossel $(17 \%, \mathrm{~N}=24)$ und Heckenbraunelle $(21 \%, \mathrm{~N}=19)$ war der Nesterfolg am geringsten. Dazwischen lagen Klappergrasmücke (29\%, N=7), Gartengrasmücke ((33\%, N=21) und Mönchsgrasmücke (36\%, N=22).

Die häufigste Verlustursache war Prädation, $54 \%$ aller Nester wurden ausgeraubt. Etwa $16 \%$ der Nester gingen durch Mahd von Randstreifen, Störungen, Witterungseinflüsse und aus ungeklärter Ursache verloren. Der Zeitpunkt des Brutbeginns, Vegetationsentwicklung, Neststandortparameter und die Flexibilität der Art bezüglich der Neststandortwahl werden als Strategien zur Vermeidung von Prädation als mögliche Ursachen für die großen Unterschiede im Bruterfolg der Arten diskutiert.

Unter Berücksichtigung von jährlicher Mortalität lässt sich aus dem Ausfliegeerfolg der Arten die Populationsentwicklung abschätzen. Es zeigte sich, dass die Populationen von Amsel, Singdrossel, Mönchsgrasmücke, Gartengrasmücke und Klappergrasmücke aufgrund geringen Bruterfolgs kleiner würden, wenn keine Zuwanderung aus anderen Habitaten erfolgt. Für die genannten fünf Arten stellen Hecken ein Sink-Habitat dar.

Goldammer, Dorngrasmücke, Heckenbraunelle und Zilpzalp erzeugen Nachwuchs, der theoretisch ausreicht, um ihre natürliche Sterblichkeit ausgleichen zu können. Hecken stellen für diese Arten ein Source-Habitat dar. 
Dieser Befund zeigt die Bedeutung von Hecken für Arten wie Goldammer und Dorngrasmücke, die stellvertretend für weitere Bewohner halboffener Heckenlandschaften hier wichtige Lebensräume haben, die auch insofern 'intakt' sind, als dass die betreffenden Arten darin überlebensfähige Populationen bilden können.

Die Populationen von Amsel, Singdrossel und einigen Grasmücken sind in Hecken von der Zuwanderung aus anderen Habitaten abhängig. Die Sink-Situation beruht für diese Arten auf Räuberdruck von Prädatoren, die in starkem Maße von der linienhaften Grundstruktur einer Hecke profitieren.

Kunstnester wurden Ende Mai, wenn die Vegetation zwischen Krautsaum und Heckenmantel am dichtesten ist, signifikant weniger ausgeraubt. Breite Hecken bieten einen besseren Prädationsschutz als schmale. In zwei Meter breiten Hecken wurden über $40 \%$, in 10 Meter breiten nur noch $20 \%$ aller ausgebrachten Nester ausgeraubt. Kunstnester wurden jedoch vor allem 'heckenweise' ausgefressen. Wenn eines der benachbarten Nester ausgeraubt war, wurde das betrachtete Nest mit 70 \%iger Wahrscheinlichkeit auch gefunden.

Auf landschaftlicher Ebene betrachtet war die Gefahr für Nester einer Hecke ausgeraubt zu werden groß, wenn die Wegedichte um das Nest hoch und die Entfernung zum nächsten Wald groß war und wenn der Gebüschmantel der Hecken schmal war.

Anhand von Fotos an Kunstnestern zeigte sich, dass die Elster der wichtigste Räuber an den Kunstnestern im April und Mai und der Eichelhäher im Juni und Juli war. Dadurch ließ sich vermuten, dass die Kunstnester von Elstern effektiv gefunden wurden, wenn Wege an der Hecke entlang führten, weil Elstern diese im Untersuchungsgebiet bevorzugt zur Nahrungssuche nutzen. Spärliche Ausprägung des Gebüschmantels erhöhte die Auffindbarkeit der Nester zusätzlich.

Auf eine saubere Trennung der Befunde aus der Kunstnest-Studie und den Ergebnissen an den Nestern der Singvögel wurde Wert gelegt, weil sich ein Kunstnest in einer Vielzahl von Charakteristika von einem echten Nest unterscheidet. 


\section{Literatur}

ANDRÉN, H. (1995): Effects of landscape composition on predation rates at habitat edges. In: Hansson, L., Fahrig, L. \& Merriam, G.: Mosaic landscapes and ecological processes. Chapman \& Hall, London.

ANDRÉN, H. \& ANGELSTAM, P. (1988): Elevated predation rates as an edge effect in habitat islands: experimental evidence. Ecology 69: 544-547.

ARNOLD, G.W. (1983): The influence of ditch and hedgerow structure, length of hedgerows, and area of woodland and garden on bird numbers on farmland. J. Appl. Ecol. 20: 731750 .

BAILLIE, S.R. (1990): Integrated population monitoring of breeding birds in Britain and Ireland. Ibis 132: 151-166.

Baillie, S.R., Green, R.E., Boddy, M. \& Buckland, S.T. (1986): An evaluation of the Constant Effort Sites Scheme. BTO Research Report 21, BTO, Thetford.

BAIRlein, F. (1978): Über die Biologie einer südwestdeutschen Population der Mönchsgrasmücke (Sylvia atricapilla). J. Orn. 119: 14-51.

BAIRLEIN, F. (1996a): Ökologie der Vögel: Physiologische Ökologie - Populationsbiologie Vogelgemeinschaften - Naturschutz. Gustav Fischer Verlag, Stuttgart.

BAIRLEIN, F. (1996b): Arbeitsanleitung für Heckenuntersuchungen 03/96. Institut für Vogelforschung "Vogelwarte Helgoland".

BAIRLEIN, F. (1997): Spatio-temporal course, ecology and energetics of western palearctic african songbird migration. European Science Foundation. Scientific Network, Summary Report 1994-1996.

BAIRLEIN, F. (2000): Nicht nur Köpfe zählen - Integriertes Monitoring als Grundlage für effektiveren Vogelschutz. Vogelschutz Heft 3/2000: 28-31.

BAIRlein, F. \& SonNTAG, B. (1994): Zur Bedeutung von Straßenhecken für Vögel. Natur und Landschaft 69: 43-48.

BAirlein, F., BAUer, H.-G. \& DORSCH, H. (2000): Integriertes Monitoring von Singvogelpopulationen. Vogelwelt 121: 217-220.

Bairlein, F., Berthold, P., Querner, U. \& Schlenker, R. (1980): Die Brutbiologie der Grasmücken Sylvia atricapilla, borin, communis und curruca in Mittel- und N-Europa. J. Orn. 121: 325-369.

BAlmer, D. \& PEACH, W. (1996): Population changes on Constant Effort Sites 1994-95. BTO News 203: 10-11.

BALMER, D. \& PEACH, W. (1998): Constant effort ringing in Britain and Ireland. EURING Newsletter 2: 18-21.

BARKow, A., BAIRlein, F. \& MÜHLEnBerg, M. (2001): Prädationsraten an Vogelnestern. In: Gottschalk, E., Barkow, A., Mühlenberg, M. \& Settele, J. (2001): Naturschutz und Verhalten. UFZ-Bericht: 111-115.

BAUER, H.-G. \& BerThold, P. (2000): Die Brutvögel Mitteleuropas - Bestand und Gefährdung. AULA-Verlag, Wiesbaden, 2. Auflage.

Begon, M.E., Townsend, C.R. \& HARPER, J.L. (1998): Ökologie. Spektrum Akademischer Verlag, Heidelberg, Berlin. 
BergmanN, H.-H. (1993): Der Buchfink: Neues über einen bekannten Sänger. AULAVerlag, Wiesbaden.

BERNDT, R. \& WINKEL, W. (1987): Zu Ortstreue, Ortswechsel und Lebensalter beim Feldsperling (Passer montanus). Vogelwelt 108: 98-105.

BERTHOLD, P. (1973): Über starken Rückgang der Dorngrasmücke Sylvia communis und anderer Singvogelarten im westlichen Europa. J. Orn. 114: 348-360.

BERTHOLD, P. (1974): Die gegenwärtige Bestandesentwicklung der Dorngrasmücke (Sylvia communis) und anderer Singvogelarten im westlichen Europa bis 1973. Vogelwelt 95: 170-183.

BERTHOLD, P. (1976): Methoden der Bestandserfassung in der Ornithologie: Übersicht und kritische Betrachtung. J. Orn. 117: 1-69.

BERTHOld, P. (1990): Vogelzug: eine Einführung und kurze aktuelle Gesamtübersicht. Wiss. Buchges. Darmstadt.

Berthold, P., Bezzel, E. \& Thielcke, G. (1980): Praktische Vogelkunde. Kilda Verlag, Greven, 2. Auflage.

Berthold, P., Fliege, G., Heine, G., Querner, U. \& Schlenker, R. (1991): Wegzug, Rastverhalten, Biometrie und Mauser von Kleinvögeln in Mitteleuropa. Vogelwarte 36, Sonderheft: 1-221.

Beshkarev, A.B., Swenson, J.E., Angelstam, P., Andrén, H. \& Blagovidov, A.B. (1994): Long-term dynamics of hazel grouse populations in source- and sinkdominated pristine taiga landscapes. Oikos 71: 375-380.

BEZZEL, E. (1982): Vögel in der Kulturlandschaft. Eugen Ulmer, Stuttgart.

Bezzel, E. (1993): Kompendium der Vögel Mitteleuropas - Passeriformes. AULA-Verlag, Wiesbaden.

BibBy, C.J., Burgess, N.D. \& HiLl, D.A. (1992): Bird Census Techniques. Academic Press Ltd., London, San Diego.

BIRKHEAD, M.E. (1981): The social behavior of the Dunnock. Ibis 123: 75-84.

BLAB, J. (1993): Grundlagen des Biotopschutzes für Tiere. Schriftenreihe für Landschaftspflege und Naturschutz 24, Kilda-Verlag, Bonn-Bad Godesberg, 4. Auflage.

BLANA, H. (1978): Die Bedeutung der Landschaftsstruktur für die Verbreitung der Vögel im Südlichen Bergischen Land. Beitr. Avifauna Rheinland 12.

BoAtman, N. (1994): Field Margins: Integrating Agriculture and Conservation. British Crop Protection Council Monograph. Farnham: BCPC.

BoDDY, M. (1993): Whitethroat Sylvia communis population studies during 1981-1991 at a breeding site on Lincolnshire coast. Ringing \& Migration 14: 73-84.

Bortz, I., LiEnerT, G.A. \& BoeHnKe, K. (1990): Verteilungsfreie Methoden in der Biostatistik. Springer-Verlag, Berlin, Heidelberg.

BORTZ, J. (1995): Statistik für Sozialwissenschaftler. Springer Verlag Berlin, 4. Auflage.

Brown, C.R. \& Brown, M.B. (1999): Fitness components associated with laying date in the cliff swallow. Condor 101: 230-245.

Brown, K. P., Moller, H., InNeS, J.\& JANSEN, P. (1998): Identifying predators at nests of small birds in a New Zeland forest. Ibis 140: 274-279. 
BusCHE, G. (1997): Bestandsentwicklung der Brutvögel des Wallhecken-Agrarlandes samt Dörfern und Städten im Westen Schleswig-Holsteins 1960-1995. Vogelwelt 118: 1132.

DIAS, P.C. (1996): Sources and sinks in population biology. Tree 11: 326-330.

DiAs, P.C. \& BLONDEL, J. (1996): Local specialisation and maladaptations in Mediterranean blue tit Parus caeruleus. Oecologia: 107: 79-86.

Dias, P.C., Meunier, F. Beltra, S. \& Cartan-Son, M. (1994): Blue tits in mediterranean habitat mosaics. Ardea 82: 363-372.

DiETRICH, F. (1903): Die schleswig-holsteinischen Knicks und ihre Bedeutung für die Vogelwelt. Abdruck in Vogel und Heimat 6 (1957): 17-22.

DÖRRIE, H. H. (2000): Ornithologischer Jahresbericht 1999 für die Region Göttingen. Naturkundl. Ber. Fauna Flora in Süd-Niedersachsen 5: 1-147.

Dow, D.D. (1978): A test fo significance for Mayfield's method of calculating nest success. Wilson Bull. 90: 291-295.

DRÖSCHMEISTER, R. (2001): Bundesweites Naturschutzmonitoring in der "Normallandschaft" mit der Ökologischen Flächenstichprobe. Natur und Landschaft 76: 58-69.

Dwenger, R. (1991): Das Rebhuhn. Neue Brehm-Bücherei, A. Ziemsen Verlag, 2. Auflage, Wittenberg Lutherstadt.

EllenBerG, H. (1996): Vegetation Mitteleuropas mit den Alpen in ökologischer, dynamischer und historischer Sicht. 5. Aufl. - Stuttgart: Ulmer.

ERDELEN, M. (1984): Bird communities and vegetation structure: I. Correlations and comparisons of simple and diversity indices. Oecologia 61: 277-284.

Evans, A (1997): The importance of mixed farming for seed-eating birds in the UK. In: Pain, D.J. \& Pienkowski, M.W. (Eds.): Farming and Birds in Europe: The common agricultiural policy and its implications for bird conservation. Academic press; San Diego, London. Chapt. 12: 331-357.

Eybert, M.-C., Constant, P. \& Lefeuvre, J.C. (1995): Effects of changes in agricultural landscape on a breeding population of Linnets living in adjacent heathland. Biol. Conserv. 74: 195-202.

FEnSKe-Crawford, T.J.\& Niemi, G.J. (1997): Predation of artificial ground nests at two types of edges in a forest-dominated landscape. Condor 99: 14-24.

FISCHER, S. \& SCHNEIDER, R. (1996): Die Grauammer Emberiza calandra als Leitart der Agrarlandschaft. Vogelwelt 117: 225-234.

FLADE, M. (1994): Die Brutvogelgemeinschaften Mittel- und Norddeutschlands. IHW-Verlag, Eching.

GAßMANN, H. \& GLÜCK, E. (1988): Avizönosen zweier Heckenlandschaften im Raum Aachen. Charadrius 24: 133-142.

GAßMANN, H. \& GLÜCK, E. (1993): Nistplatzwahl und Bruterfolg von Vögeln in Hecken. Vogelwelt 114: 136-147.

GATES, J.E. \& GYSEL, L.W. (1978): Avian nest dispersion and fledging success in field-forest ecotones. Ecology 59: 871-883.

GEORGE, K. (1996a): Deutsche Landwirtschaft im Spiegel der Vogelwelt. Vogelwelt 117: $187-197$. 
GlutZ Von Blotzheim, U.N. \& BAuER, K.M. (1985): Handbuch der Vögel Mitteleuropas Bd. 10/I: Passeriformes (1. Teil). AULA-Verlag, Wiesbaden.

Glutz Von Blotzheim, U.N. \& Bauer, K.M. (1988a): Handbuch der Vögel Mitteleuropas Bd. 11/I: Passeriformes (2. Teil) Turdidae. AULA-Verlag, Wiesbaden.

Glutz Von Blotzheim, U.N. \& BAuer, K.M. (1988b): Handbuch der Vögel Mitteleuropas Bd. 11/II: Passeriformes (2. Teil) Turdidae. AULA-Verlag, Wiesbaden.

Glutz VOn Blotzheim, U.N. \& BAuer, K.M. (1991a): Handbuch der Vögel Mitteleuropas Bd. 12/I: Passeriformes (3. Teil) Sylviidae. AULA-Verlag, Wiesbaden.

Glutz Von Blotzheim, U.N. \& BAuer, K.M. (1991b): Handbuch der Vögel Mitteleuropas Bd. 12/II: Passeriformes (3. Teil) Sylviidae. AULA-Verlag, Wiesbaden.

Glutz von Blotzheim, U.N. \& BAuer, K.M. (1997a): Handbuch der Vögel Mitteleuropas Bd. 14/I: Passeriformes (5. Teil). AULA-Verlag, Wiesbaden.

Glutz von Blotzheim, U.N. \& BAuer, K.M. (1997b): Handbuch der Vögel Mitteleuropas Bd. 14/II: Passeriformes (5. Teil). AULA-Verlag, Wiesbaden.

Glutz Von Blotzheim, U.N. \& BAueR, K.M. (1997c): Handbuch der Vögel Mitteleuropas Bd. 14/III: Passeriformes (5. Teil). AULA-Verlag, Wiesbaden.

GNIELKA, R. (1992): Möglichkeiten und Grenzen der Revierkartierungsmethode. Vogelwelt 113: $231-240$.

Gottschalk, E., Barkow, A., MühlenberG, M. \& Settele, J. (2001): Naturschutz und Verhalten. UFZ-Bericht 2/2001.

GRAJETZKY, B. (1993): Bruterfolg des Rotkehlchens Erithacus rubecula in Hecken. Vogelwelt 114: 232-240.

GRAJETZKY, B. (2000): Das Rotkehlchen: Zeit- und Energiekonflikte - ein Kleinvogel findet Lösungen. Wiebelsheim, Aula-Verlag.

Green, R.E., Osborne, P.E. \& SEARS, E.J. (1994): The distribution of passerine birds in hedgerows during the breeding season in relation to characteristics of the hedgerow and adjacent farmland. Appl. Ecol. 31: 677-692.

GREEN, R.F. (1977): Do more birds produce fewer young? A comment on Mayfield's measure of nest success. Wilson Bull. 89: 173-175.

HAHN, V. (1966): Der Vogelbestand einer Wiesen-Knick-Landschaft bei Wedel (Holstein). Hamb. Avifaun. Beitr. 3: 124-165.

HALUPKA, K. (1998): Partial nest predation in altrical birds selects for the accelerated development of young. J. Avian Biol. 29: 129-133.

HANSKI, I. \& GILPIN, M.E. (1997): Metapopulation Biology - Ecology, genetics, and evolution. Academic Press, San Diego, London.

HANSSON, L., FAHRIG, L. \& MERRIAM, G. (1995): Mosaic landscapes and ecological processes. Chapman \& Hall, London.

HAUBER, M.E. (1998): Single-egg removal from an artificial nest by the Gray Catbird. Wilson Bull. 110: 426-429.

HeitKamp, U. (1981): Die Vogelpopulationen einer Saumbiozönose am Stadtrand von Göttingen in den Jahren 1963 bis 1968. Unveröffentlicht.

Helbig, A.J. \& DierschKe, V. (2000): Zugvogelkalender. Ornithologen Kalender 2001Jahrbuch für Vogelkunde und Vogelschutz: 128-150. 
HinsLey, S.A. \& BELLAMY, P.E. (2000): The influence of hedge structure, management and landscape context on the value of hedgerows to birds: a review. J. Environmental Manag. 60: 33-49.

Hinsley, S.A., Bellamy, P.E., Newton, I., Sparks, T.H. (1995): Habitat and landscape factors influencing the presence of individual breeding bird species in woodland fragments. J. Avian Biol. 26: 94-104.

HoOPER, M.D. (1994): In: Watt, T.A. \& Buckley, G.P. (eds.): Hedgerow management and nature conservation. British Ecological Society Conservation Ecology Group, Wye College Press: 58-79.

Hovestadt, T., Roeser, J. \& MÜHLEnBerG, M. (1991): Flächenbedarf von Tierpopulationen. Forschungszentrum Jülich.

Huhta, E., MAPPES, T. \& JOKIMÄKI, J. (1996): Predation on artificial ground nests in relation to forest fragmentation, agricultural land and habitat structure. Ecography 19: 85-91.

IMS, R.A. \& YACCOZ, N.G. (1997): Studying transfer processes in metapopulations: emigration, migration, and colonization. In: Hanski, I. \& Gilpin, M.E.: Metapopulation Biology - Ecology, genetics, and evolution. Academic Press, San Diego, London.

JAHN, B. (2000): Untersuchungen zum Bruterfolg der Elster Pica pica: Einflüsse des Angebots an Nahrungsflächen und benachbarter Corvidennester. Diplomarbeit, unveröffentlicht.

JAKOBER, H. \& STAUBER, W. (1981): Habitatansprüche des Neuntöters Lanius collurio - Ein Beitrag zum Schutz einer gefährdeten Art. Ökol. Vögel 3: 223-247.

JAKOBER, H. \& StAubeR, W. (1987): Habitatansprüche des Neuntöters. Beih. Veröff. Naturschutz und Landespfl. Baden-Württ. 48: 25-55.

JEDICKE, E. (1994): Biotopverbund: Grundlagen und Maßnahmen einer neuen Naturschutzstrategie. 2. Auflage Stuttgart: Ulmer.

Jedicke, E. (1997): Die Roten Listen - Gefährdete Pflanzen, Tiere, Pflanzengesellschaften und Biotoptypen in Bund und Ländern. Ulmer Verlag Stuttgart.

JeNNi, L. \& WinKLER, R. (1994): Moult and ageing of European passerines. Academic Press, London.

JoHnsOn, D.H. (1979): Estimating nest success: The Mayfield method and an alternative. Auk 96: 651-661.

KAISER, W. (1997): Telemetrische Untersuchungen zur Habitatnutzung des Rebhuhns im Raum Feuchtwangen. Schriftenreihe des Bayerischen Landesamtes für Umweltschutz, Heft 142. Beiträge zum Artenschutz 21: Naturschutz in der Agrarlandschaft.

Kaule, G. (1991): Arten- und Biotopschutz. Ulmer Verlag, Stuttgart, 2. Auflage.

Keyser, A.J., Hill, G.E. \& Soehren, E.C. (1998): Effects of forest fragment size, nest density, and proximity to edge on the risk of predation to ground-nesting passerine birds. Cons. Biol. 12: 986-994.

KirChHOFF, K. \& IHSSEN, G. (1972): Der Brutvogelbestand eines Wiesen-Feldmarkgebietes mit Knicks in Hamburg-Hummelsbüttel in den Jahren 1968 und 1969. Hamb. Avifaun. Beitr. 10: 177-192.

KLEIN, W. (1979): Die Vogelbestände auf einer landwirtschaftlich genutzten Fläche in der südöstlichen Wetterau 1976-1978/79. Luscinia 44: 41-88. 
KOHLER (1975): Literaturauswertung von Mortalitätsraten bei Sylviiden. Examensarbeit Universität Bonn, unveröffentlicht.

KREBS, J.R. (1971): Territory and breeding density in the Great Tit Parus major. Ecology 52: $2-22$.

KuJAVA, K. (1994): Influence of land-use change within agricultural landscapes on the abundance and diversity of breeding bird communities. In: Ryszkowski, L. \& Balazy, S. (Eds.): Funktional appraisal of agricultural landscape in Europe. Pp. 183-196.

KuJAVA, K. (1997): Relationships between the structure of mid-field woods and their breeding bird communities. Acta Orn. 32: 175-184.

LACK, P.C. (1987): The effects of severe hedge cutting on a breeding bird population. Bird Study 34: 139-146.

LACK, P.C. (1988): Hedge intersections and breeding bird distribution in farmland. Bird Study 35: 133-136.

LAKHANI, K.H. (1994): The importance of field margin attributes to birds. In: Field Margins: Integrating Agriculture and Conservation. British Crop Protection Council Monograph no. 58. (N. Boatman, ed.), pp. 77-84. Farnham: BCPC.

Lille, R. (1996): Zur Bedeutung von Bracheflächen für die Avifauna der Agrarlandschaft: Eine nährungsökologische Studie an der Goldammer Emberiza citrinella. Paul Haupt Verlag, Stuttgart, Bern.

LOVEJOY, T.E. (1974): Bird diversity and abundance in amazon forest birds. Living Bird 13: 127-191.

MacAthur, R.H. \& Wilson, E.O. (1967): The Theory of Island Biogeography. Princeton, NJ, Princeton University Press.

MacDonald, D.W. \& Johnson, P.J. (1995): The relationship between bird distribution and the botanical and structural characteristics of hedges. J. Appl. Ecol. 32: 492-505.

MADER, H.-J. (1988): Effects of increased spatial heterogeneity on the biocenosis in rural landscapes. Ecological Bulletins 39: 169-179.

MADER, H.-J., KLÜPPEL, R. \& OVERMEYER, H. (1986): Experimente zum Biotopverbundsystem - tierökologische Untersuchungen an einer Anpflanzung. Schriftenreihe für Landschaftspflege und Naturschutz 27, Bonn-Bad Godesberg.

MAJOOR, F. (1996): Constant Effort Sites Project 1995-1996. Op Het Vikentower 82: 14-20.

MAJOR, R.E. \& KENDAL, C.E. (1996): The contribution of artificial nests experiments to understanding avian reproductive success: A review of methods and conclusions. Ibis 138: 298-307.

Marchant, J.H. (1983): BTO Common Birds Census Instructions. BTO, Tring, Herts.

Marchant, J.H., Hudson, R., Carter, S.P. \& Whittington, P.A. (1990): Population trends in British breeding birds. BTO, Tring.

MARINI, M.A. \& MELO, C. (1998): Predators of quail eggs, and the evidence of the remains: implications for the nest predation studies. Condor 100: 395-399.

MARINI, M.A., Robinson, S.K. \& HeSKE, E.J. (1995): Edge effects on nest predation in the shawnee national forest, southern Illinois. Biol. Cons. 74: 203-213.

Marini, M.A. \& Weale, M.E. (1997): Density- and frequency-dependent predation of artificial bird nests. Biol. J. of the Linnean Society. 
MARTIN, T.E. (1988): Habitat and area effect on forest bird assemblages: is nest predation an influence? Ecology 69: 74-84.

MARTIN, T.E. (1992): Interactions of nest predation and food limitation in reproductive strategies. Current Ornithology 9: 163-197.

MARTIN, T.E. (1993a): Nest predation among vegetation layers and habitat types: revising the dogmas. Am. Nat. 141: 897-913.

MARTIN, T.E. (1993b): Evolutionary determinants of clutch size in cavity-nesting birds: nest predation or linited breeding opportunities. Am. Nat. 142: 937-946.

MARTIN, T.E. (1993c): Nest predation and nest sites. Bioscience 43:523-532.

MARTIN, T.E. \& PINGJUN, L. (1992): Life history traits of open-vs. cavity-nesting birds. Ecology 73: 579-592.

MARTIN, T.E. \& ROPER, J.J. (1988): Nest predation and nest-site selection of a western population of the Hermit Thrush. The Cooper Ornithological Society 90: 51-57.

MATESSI, G. \& BogliAnI, G. (1999): Effects of nest features and surrounding landscape on predation rates of artificial nests. Bird Study 46: 184-194.

MAYFIELD, H.F. (1961): Nesting success calculated from exposure. Wilson Bull. 73: 255-261.

MAYFIELD, H.F. (1975): Suggestions for calculating nest success. Wilson Bull. 87: 456-466.

MÄCK, U. \& JÜRGENS, M.-E. (1999): Aaskrähe, Elster und Eichelhäher in Deutschland. Bericht über den Kenntnisstand und die Diskussionen zur Rolle von Aaskrähe (Corvus corone), Elster (Pica pica) und Eichelhäher (Garrulus glandarius) im Naturhaushalt sowie die Notwendigkeit eines Bestandsmanagements. Bundesamt für Naturschutz, Bonn.

MefFe, G.K. \& CARroll, C.R. (1994): Principles of Conservation Biology. Sinauer Assosiates, Inc., Sunderland, Massachusetts.

MOLES, R.T. \& BREEN, J. (1995): Long-term change within lowland farmland bird communities in relation to field boundary attributes. Biology and Environment: Proceedings of the Royal Irish Academy 95B: 203-215.

MøLlER, A. P. (1989): Nest site selection across field-woodland ecotones: the effect of nest predation. Oikos 56: 240-246.

MøLleR, A. P. (1995): Development stability and ideal despotic distribution of blackbirds in a patchy environment. Oikos 72: 228-234.

MøLler, A.P. (1994): Sexual selection and the barm swallow. Oxford University Press, Oxford.

MÜHLENBERG, M. (1982): Artenverlust - trotz ökologischer Planung. Natur und Landschaft 57: 295-296.

MÜHLEnBerG, M. (1993): Freilandökologie. 3. Auflage, UTB 595, Quelle \& Meyer, Heidelberg.

MÜHLENBERG, M. (1998): Populationsbiologie und Gefährdung: das Zielartenkonzept. Artenschutzreport 8: 9-14.

MÜHLenberG, M. \& Hovestadt T. (1992): Das Zielartenkonzept. NNA - Berichte 5: 36-39.

MÜHLEnBERG, M. \& SLOWIK, J. (1997): Kulturlandschaft als Lebensraum. UTB Quelle und Meyer, Wiesbaden, 312 S.. 
MüLLER, G. (1989): Wallhecken - Entstehung, Pflege, Neuanlage am Beispiel der Gemeinde Ganderkesee und allgemeine Hinweise zu Wallhecken im nordwestdeutschen Raum. BSH Verlag Wardenburg.

MÜLLER, J. (1989): Landschaftsökologische und -ästhetische Funktionen von Hecken und deren Flächenbedarf in Süddeutschen Intensiv-Agrarlandschaften. Ber. ANL 13: 3-58.

MÜLlER, J. (1990): Bericht über Funktionen von Hecken als Beispiel einer ganzheitlichen Ökosystembetrachtung. In: Jahrbuch der Akademie der Wissenschaften zu Berlin 1989, S.44-53.

MURRAY JR., B.G. (1997): Net shyness in the Wood Thrush. J. Field Ornithol. 68: 348-357.

NiEmuth, N.D.\& Boyce, M.S. (1997): Edge-related nest losses in Wisconsin pine barrens. J. Wildl. Manage. 61: 1234-1239.

Nitsche, G. \& Plachter, H. (1987): Atlas der Brutvögel Bayerns 1979-1983. Ornith. Ges. i. Bayern, Bayerisches Landesamt für Umweltschutz München.

O'ConNOR, R.J. \& SHrubB, M. (1986): Farming and birds. Cambridge University Press, Cambridge.

OELKE, H. (1980): Quantitative Untersuchungen: Siedlungsdichte. In: Berthold et al. (1980): Praktische Vogelkunde. Kilda Verlag, Greven.

OelKe, H. (1981): Limitations of the mapping method. Avian Biol. 6: 114-118.

OELKe, H. (1985): Vogelbestände einer niedersächsischen Agrarlandschaft. Vogelwelt 106: 246-255.

Oelke, H., KuKliK, H.-W. \& Nielitz, U. (1992): Die Vögel der Börden im nordwestlichen und nordöstlichen Harzvorland. Beitr. Naturk. Niedersachs. 45: 153-176.

OSBORNE, P. (1984): Bird numbers and habitat characteristics in farmland hedgerows. J. Appl. Ecol. 21: 63-82.

Pain, D.J. \& Pienkowski, M.W. (1997): Farming and Birds in Europe: The common agricultiural policy and its implications for bird conservation. Academic press; San Diego, London.

PANEK, M. (1994): The effect of land-use changes on populations of Partridge (Perdix perdix) in Poland. In: Ryszkowski, L. \& Balazy, S. (Eds.): Funktional appraisal of agricultural landscape in Europe: 197-203.

PARISH, T., LAKHANI, K.H. \& SPARKS, T.H. (1994): Modelling the relationship between bird populaton variables and hedgerow and other field margin attributes. I. Species richness of winter, summer and breeding birds. J. Appl. Ecol. 31: 764-775.

PARISH, T., LAKHANI, K.H. \& SpARKS, T.H. (1995): Modelling the relation between bird popultion variables and hedgerow and other field margins attributes. II. Abundance of inidividual species and groups of similar species. J. Appl. Ecol. 32: 362-371.

PATON, P.W. (1994): The effect of edge on avian nest success: How strong is the evidence? Cons. Biol. 8: 17-26.

PEACH, W.J., Buckland, S.T. \& Baillie, S.R. (1996): The use of constant effort mist-netting to measure between -year changes in the abundance and productivity of common passerines. Bird Study 43: 142-156.

PoTTS, D. (1997): Cereal farming, pesticides and grey partridges. In: Pain, D.J. \& Pienkowski, M.W. (Eds.): Farming and Birds in Europe: The common agricultiural 
policy and its implications for bird conservation. Academic press; San Diego, London. Chapt. 6: 150-177.

PuCHSTEIN, K. (1980): Zur Vogelwelt der schleswig-holsteinischen Knicklandschaft mit einer ornitho-ökologischen Bewertung der Knickstrukturen. Corax 8: 62-106.

PULLIAM, H.R. (1988): Sources, sinks, and population regulation. Am. Nat. 132: 652-661.

Pulliam, H.R. \& DunNing, J.B. (1994): Demographic Processes - Population Dynamics on Heterogeneous Landscapes. In: Meffe, G.K. \& Carroll, C.R. (1994): Principles of Conservation Biology. Sinauer Assosiates, Inc., Sunderland, Massachusetts, 600 pp.

RANDS, M.R.W. (1985): Pesticide use on cereals and the survival of grey partridge chicks: a field experiment. J. Appl. Ecol. 22: 49-54.

RICKLEFS, R.E. (1969): An analysis of nesting mortality in birds. Smithson. Contrib. Zool. 9: $1-48$.

RICKLEFS, R.E. (1989): Nest predation and the species diversity of birds. Tree 4: 184-186.

RIDINGTON, R. \& GosLeR, A.G. (1995): Differences in reproductive success and parental qualities between habitats in the Great Tit Parus major. Ibis 137: 371-378.

Ringler, A., Roßmann, D. \& Steidl, I. (1997): Hecken und Feldgehölze Landespflegekonzept Bayern, Band II.12. Hrsg.: Bayerisches Staatsministerium für Landesentwicklung und Umweltfragen (StMLU) und Bayerische Akademie für Naturschutz und Landespflege (ANL), München.

ROBERTSON, R.J. (1972): Optimal niche space of the redwinged blackbird (Agelaius phoeniceus). I. Nesting success in marsh and upland habitat. Can. J. Zool. 50: 247263.

RothHAupt, G. (1997): Populationsgefährdungsanalyse am Raubwürger (Lanius excubitor L.). Cuvillier Verlag Göttingen.

RYSZKOWSKI, L. \& BALAZY, S. (1994): Funktional appraisal of agricultural landscape in Europe. Research Centre for agricultural and forest environment. Polish acedemy of science, Poznán.

SACHS, L. (1992): Angewandte Statistik. Springer Verlag Berlin, 7. Auflage.

SANTOS, T. \& TELLERÍA, J.L. (1992): Edge effects on nest predation in Mediterranean fragmented forests. Biol. Cons. 60: 1-5.

SARACCO, J.F., COLlazO, J.A. (1999): Predation and artificial nests along three edge types in a North Carolina bottomland hardwood forest. Wilson Bull. 111: 541-549.

SARgent, R.A., Kilgo, J.C., ChapMAn, B.R. \& Miller, K.V. (1998): Predation of artificial nests in hardwood fragments enclosed by pine and agricultural habitats. J. Wildl. Manag. 62: 1438-1442.

SCOTt Morales, L. (1997): Zur Bedeutung von Extensivierung in der Landnutzung für die Goldammer (Emberiza citrinella). Wissenschaft und Technik Verlag Gross, Berlin.

SHALAWAY, S.D. (1985): Fencerow management for nesting birds in Michigan. Wildlife Society Bull. 13: 302-306.

SchAEFER, M. (1992): Wörterbücher der Biologie: Ökologie. 3. Auflage, Gustav Fischer Verlag, Jena.

SCHAEFER, T. (in prep.): Nest Predation and renesting in the blackcap. 
SCHÖNFELD, M. (1984): Der Fitislaubsänger. Neue Brehm-Bücherei, A. Ziemsen Verlag, 2. Auflage, Wittenberg Lutherstadt.

Siriwardena, G. M., Baillie, S., CRICK, H.Q.P. \& Wilson, J. D. (2000): The importance of variation in the breeding performance of seed-eating birds in determining their population trends on farmland. J. Appl. Ecol. 37: 128-148.

SNOW, B.K. \& SNOW, D.W. (1982): Territory and social organization in a population of Dunnocks. J. Yamashina Inst. Orn. 14: 281-292.

SPARKS, T.H., PARISH, T. \& HinSLEY, S.A. (1996): Breeding birds in field boundaries in an agricultural landscape. Agriculture, Ecosystems and Environment 60: 1-8.

StACEY, P.B., Johnson, V.A. \& TAPER, M.L. (1997): Migration within metapopulations: the impact upon local population dynamics. In: Hanski, I. \& Gilpin, M.E.: Metapopulation Biology - Ecology, genetics, and evolution. Academic Press, San Diego, London.

Streeter, D., Richardson, R. \& DreYeR, W. (1985): Hecken: Lebensadern der Landschaft. Gerstenberg Verlag, Hildesheim.

SuAReZ, A.v., Pfennig, K.S. \& Robinson, S.K. (1997): Nesting success of a disturbancedependent songbird on different kinds of edges. Cons. Biol. 11: 928-935.

Svensson, L. (1992): Identification guide to European passerines. BTO, The Nunnery, Norfolk, England.

TinBergen, J.M. (1990): Nestling weight and survival in individual great tit (Parus major). J. Animal Ecol. 59: 1113-1127.

TiSCHLER, W. (1948a): Über die Bedeutung der Schädlingsfauna in den Wallhecken Schleswig-Holsteins. Beitr. z. Agrarwiss. 2: 50-55.

TischleR, W. (1948b): Biozönotische Untersuchungen an Wallhecken. Zool. Jb. Abt. Syst. Ökol. und Geogr. 77: 284-400.

TisCHLER, W. (1948c): Zum Geltungsbereich der biozönotischen Grundeinheiten. Forschung und Fortschritte 19/20: 235-238.

TiSCHLER, W. (1959): Vergleichend-biozönotische Untersuchungen an Waldrand und Feldhecke. Zool. Anzeiger: 1000-1015.

TischleR, W. (1990): Ökologie der Lebensräume. UTB für Wissenschaft, G. Fischer Verlag.

TUCKER, G.M. \& HEATH, M.F. (1994): Birds in Europe: Their conservation status. Birdlife International, Cambridge.

VAn HoRne, B. (1983): Density as a misleading indicator of habitat quality. J. Wildlife Manag. 47: 893-901.

ViCKERY, P.D., HUNTER, JR., M.L. \& WeLLS, J.V. (1992a): Evidence of incidental nest predation and its effects on nests of threatened grassland birds. Oikos 63: 281-288.

Vickery, P.D., HunTER, JR., M.L. \& Wells, J.V. (1992b): Is density an indicator of breeding success? Auk 109: 706-710.

VICKERY, P.D., HunTER, JR., M.L. \& Wells, J.V. (1992c): Use of a new reproductive index to evaluate relationship between habitat quality and breeding success. Auk 109: 697705.

Vogel, K., Vogel, B., Rothhaupt, G. \& Gottschalk, E. (1996): Einsatz von Zielarten im Naturschutz. - Auswahl der Arten, Methode von Populationsgefährdungsanalyse und Schnellprognose, Umsetzung in der Praxis. Naturschutz und Landschaftsplanung 28: 179-184. 
WATKInson, A.R. \& Sutherland, W.J. (1995): Sources, sinks and pseudo-sinks. Journal of Animal Ecol. 64: 126-130.

WATT, T.A. \& BUCKLEY, G.P. (1994): Hedgerow management and nature conservation. British Ecological Society Conservation Ecology Group, Wye College Press.

WeBER, H.E. (1967): Über die Vegetation der Knicks in Schleswig-Holstein. Mitt. Arb.-gem. Floristik Schl.-Holst. und Hamburg 15: 1-196.

WILSON, R.R. \& COOPER, R.J. (1998): Acadian flycatcher nest placement: does placement influence reproductive success? Condor 100: 673-679.

WinKEL, W. (1993): Zum Migrationsverhalten von Kohl- und Blaumeise. In: Bairlein, F.: Jber. Institut Vogelforschung 1: 9.

WinKEL, W. \& FrANTZEN, M. (1989): Ortstreue, Emigration und Lebensalter von Kohlmeisen (Parus major) im Braunschweiger Raum. Vogelwarte 35: 64-79.

Winkel, W. \& Frantzen, M. (1991): Zur Populationsdynamik der Blaumeise (Parus caeruleus): Langfristige Studien bei Braunschweig. J. Orn. 132: 81-96.

Winstanely, D., SPENCER, R. \& Williamson, K. (1974): Where have all the Whitethroats gone? Bird Study 21: 1-14.

Witt, K., Bauer, H.-G., Berthold, P., Boye, P., Hüppop, O. \& Knief, W. (1996): Rote Liste der Brutvögel Deutschlands. Ber. Vogelsch. 34: 11-35.

ZIMMERLI, E. (1979): Vogelreichtum waadtländischer Hecken. Vögel der Heimat 49: 181.

ZwÖLFER, H., BAuER, G. \& Heusinger, G. (1981): Ökologische Funktionsanalyse von Feldhecken - Tierökologische Untersuchung über Struktur und Funktion biozönotischer Komplexe. Schlußbericht des Lehrstuhls Tierökologie (Universität Bayreuth) an das Bayerische Landesamt für Umweltschutz in München.

Zwölfer, H., BAuer, G., Heusinger, G., Stechmann, D. (1984): Die tierökologische Bewertung von Hecken. Beiheft 3, Teil 2 zu den Berichten der Akademie für Naturschutz und Landschaftspflege. 


\section{Dank}

Zur Entstehung dieser Arbeit haben viele liebe Menschen beigetragen, denen ich an dieser Stelle herzlich danken möchte.

An erster Stelle möchte ich Herrn Prof. Dr. Franz Bairlein danken, der mich mit der Auswertung des Heckenprogramms betraut hat, mich bei der Bewerbung um ein Stipendium erfolgreich unterstützt und letztendlich das Thema der Dissertation vergeben und die Arbeit betreut hat. Darüber hinaus danke ich für Rat und Kritik in vielen fachbezogenen Gesprächen, die mir immer weiter geholfen haben.

Herrn Prof. Dr. Michael Mühlenberg danke ich für die breite Unterstützung, die ich zur Durchführung der Arbeit durch ihn und das Zentrum für Naturschutz erhalten habe. Ohne die Infrastruktur und die Freiheiten, die ich vor allem während der Freilandarbeit eingeräumt bekam, wäre die Arbeit so nicht durchführbar gewesen.

Herrn Prof. Dr. Matthias Schaefer danke ich für die freundliche Übernahme des Koreferats und für die positive Begutachtung meines Dissertationsvorhabens, die mir die Ausführung der Arbeit erst ermöglicht hat.

Mein Dank gilt der Deutschen Bundestiftung Umwelt, die mich mit der Vergabe eines Stipendiums unterstützt hat. Insbesondere möchte ich hier Herrn Dr. M. Kleinke nennen, der mein Fachbetreuer von Seiten der Stiftung war.

Ganz herzlicher Dank gebührt meiner Familie, die mich mit aller Liebe und vielem mehr bei meinem Vorhaben unterstützt hat.

Dir Siemone danke ich für all das was Du für uns in der ganzen Zeit geleistet hast, für die vielen Kilometer, die Du dafür gefahren bist, die Unterstützung die Du mir gegeben hast und dass Du mir immer das Gefühl vermittelt hast, dass ich mich auf Deine Hilfe verlassen kann.

Dann möchte ich ganz besonders Dr. Eckhard Gottschalk und Thomas Schaefer für beinahe penetrante Bereitschaft zur Hilfe und Unterstützung für alle Belange in meiner Arbeit und darüber hinaus danken. Ohne Stefan Suchi hätte ich mich wahrscheinlich gar nicht getraut, die Arbeit anzunehmen. Stefan, vielen Dank für Deine Hilfe, wenn ich statistisch abgesicherte Probleme hatte. Angi Görlich möchte ich für die vielfältige Unterstützung beim Aufbau der Datenbank, bei der Dateneingabe und bei der Feldarbeit danken.

Ebba Koglin, Siemone Anders, Stefan Suchi und C. Mac Stiegel sei ganz herzlich für die vielen Mühen bei der Überprüfung von Orthographie, Layoutverbesserungen und der Endformatierung, also der ganzen Last-Minute-Arbeit gedankt. 
Dankbar bin ich vor allem für die großartige Hilfe zu Beginn der Feldarbeit. Hier möchte ich Uli Gottschalk, Monika Meiners, Lilly Nockemann, C. Mac Stiegel, Hans-Georg Stroh, Eva Wabnitz und Frank Wichmann sowie Sandro Chubinishvili für die teilweise auch recht harte Arbeit danken.

Für harte Arbeit möchte ich auch unbedingt Axel Rohrig danken, der mir meine betagten Passate in kürzester Zeit immer wieder schnell für den Einsatz bereit machen konnte. Bis auf eine Ausnahme jedenfalls und auch da war sofort Ersatz da. Super!

Für die Unterstützung bei der Feldarbeit, sei es Netzfang, Kartierungen oder die Arbeit zur Durchführung der Prädationsexperimente danke ich:

Fabian Bindrich, Markus Deutsch, Andreas Dinkelmeyer, Angi Görlich, Timm Kabus, Lilly Nockemann, Bärbel Schäfer, Stefanie Voigt und Frank Wichmann (Netzfang);

Siemone Anders, Werner Beeke, Markus Brändel, Imme Dirks, Anna Fellechner, Volker Gaebele, Alexander Hülper, Petra Hülper, Ulrike Köhler, Katrin Lehmann, Silke Malorny, Margaretha Pangau, Dag Pfannenschmidt, Cornelia Preidel, Cosima Reiss, Thomas Schaefer, Kerstin Schniederkötter, Nicole und Thomas (Prädation);

Fabian Bindrich, Markus Deutsch und Frank Wichmann (Kartierungen und Beobachtungen).

Vielen Dank Elisabeth Opielka für die Heilung meines PC, wenn der anfing, seiner eigenen Logik zu folgen und ich dieser nicht mehr. Danke Monika Deseniß, Silvia Thies und Peter Melchert für die vielen kleinen Dienste, die Ihr im Laufe der Jahre für mich geleistet habt.

Jochen Dierschke, Martin Schaefer, Gregor Scheiffarth und Veronika Schmidt danke ich für die nette Atmosphäre im Arbeitszimmer der 'Experten' und die schnellen kleinen Hilfen und Tipps, die mir oft geholfen und viel Zeit und Frust gespart haben.

Ein besonderer Dank sei Frau A. Epting, die mir viel Arbeit mit der Eingabe von Daten für das Heckenprogramm abgenommen hat und Herrn Rolf Nagel, der die Technik für die Kameras ausgestüftelt und gebaut hat sowie an die immer freundlichen und hilfsbereiten Frau M. Enxing, Frau E. Wiechmann und Frau Karin und Herrn Andreas Reents am Institut für Vogelforschung. Ebenso danke ich Herrn Walter Foken und Gerhard Thesing, die meine Netz- und Ringbestellungen immer postwendend erledigt haben und meine Beringungslisten entziffern mussten.

Des Weiteren möchte ich Herrn Dr. W. Winkel und seiner Frau sowie Herrn Wimmer für den Besuch und die Gespräche im Gelände und die umfangreiche Literaturgabe danken. Ebenso Herrn Prof. Dr. H. Oelke, dem ich nicht nur im Hinblick auf Literatur zu Dank verpflichtet 
bin. Ich möchte Herrn Prof. Dr. P. Berthold für die Einladungen zu Vorträgen an die Vogelwarte Radolfzell, Forschungsstelle für Ornithologie der Max Planck Gesellschaft, und die anschließenden Diskussionen danken. In diesem Zusammenhang sei Wolfgang Forstmeier und Wolfgang Fiedler für die Anregungen und Auswertungstipps gedankt.

Gedankt sei an dieser Stelle abschließend allen ehrenamtlichen Beringern der deutschen Vogelwarten, die am Heckenprogramm teilgenommen haben, für die enorme Mühe und Zeit, die sie zur Erhebung der Daten aufgewandt haben:

Herrn H. Diry, Herrn S. Fischer, Herrn J. Giese, Herrn H. Giessler, Herrn B. Grajetzky, Herrn K. Greve, Herrn J. Hadasch, Herrn S. Hausmann, Herrn W. Heppe, Herrn H.-J. Hoff, Herrn H. Hollenbach, Herrn H. Kannhäuser, Frau W. Laich, Herrn, K.H. Lang, Herrn S. Martens, Herrn M.A. Neumann, Herrn H.W. Patt, Herrn Schemmel, den Herren S. \& J. Schlegel, Herrn H. Schwarze, Herrn V. Semrau, Herrn U. Eidam und der Gruppe Untermain und Herrn Vohwinkel. 


\section{Anhang}

Tab. 44: Gesamtzahl der Brutpaare (BP) und Dominanz (D) der Programmvögel über fünf Jahre in 33 Hecken.

\begin{tabular}{r|l|c|c|} 
& \multicolumn{1}{|c|}{ ART } & BP & D \\
\hline 1 & Mönchsgrasmücke & 357 & 9,8 \\
2 & Zilpzalp & 347 & 9,5 \\
3 & Amsel & 307 & 8,4 \\
4 & Gartengrasmücke & 243 & 6,7 \\
5 & Goldammer & 235 & 6,5 \\
6 & Heckenbraunelle & 225 & 6,2 \\
7 & Kohlmeise & 186 & 5,1 \\
8 & Buchfink & 176 & 4,8 \\
9 & Dorngrasmücke & 162 & 4,4 \\
10 & Rotkehlchen & 154 & 4,2 \\
\hline 11 & Fitis & 136 & 3,7 \\
12 & Blaumeise & 120 & 3,3 \\
13 & Sumpfrohrsänger & 115 & 3,2 \\
14 & Grünling & 105 & 2,9 \\
15 & Singdrossel & 102 & 2,8 \\
16 & Feldsperling & 96 & 2,6 \\
17 & Gelbspötter & 88 & 2,4 \\
18 & Klappergrasmücke & 73 & 2,0 \\
19 & Zaunkönig & 72 & 2,0 \\
\hline
\end{tabular}

\begin{tabular}{l|l|c|c|}
\multicolumn{1}{|c|}{ ART } & BP & D \\
\hline 20 & Neuntöter & 54 & 1,5 \\
21 & Star & 42 & 1,2 \\
22 & Nachtigall & 27 & 0,7 \\
23 & Wacholderdrossel & 27 & 0,7 \\
24 & Weidenmeise & 23 & 0,6 \\
25 & Girlitz & 21 & 0,6 \\
26 & Gimpel & 20 & 0,5 \\
27 & Sumpfmeise & 20 & 0,5 \\
28 & Stieglitz & 19 & 0,5 \\
29 & Schwanzmeise & 15 & 0,4 \\
\hline 30 & Bluthänfling & 14 & 0,4 \\
31 & Gartenrotschwanz & 13 & 0,4 \\
32 & Trauerschnäpper & 8 & 0,2 \\
33 & Gartenbaumläufer & 8 & 0,2 \\
34 & Feldschwirl & 8 & 0,2 \\
35 & Grauschnäpper & 7 & 0,2 \\
36 & Kernbeißer & 7 & 0,2 \\
37 & Kleiber & 6 & 0,2 \\
38 & Baumpieper & 4 & 0,1 \\
\hline
\end{tabular}


Tab. 45: Übersicht der Beringungszahlen von adulten und juvenilen Erstfängen und Nestlingen sowie Dominanz [\%] jeder Art für das betreffende Alter, Abk. im Text, unter 'Habit' sind Arten gekennzeichnet, die aufgrund ihrer Habitatwahl oder Verbreitung nur unter besonderen Umständen in Hecken siedeln, siehe dazu 'Auswahl der Programmvögel'.

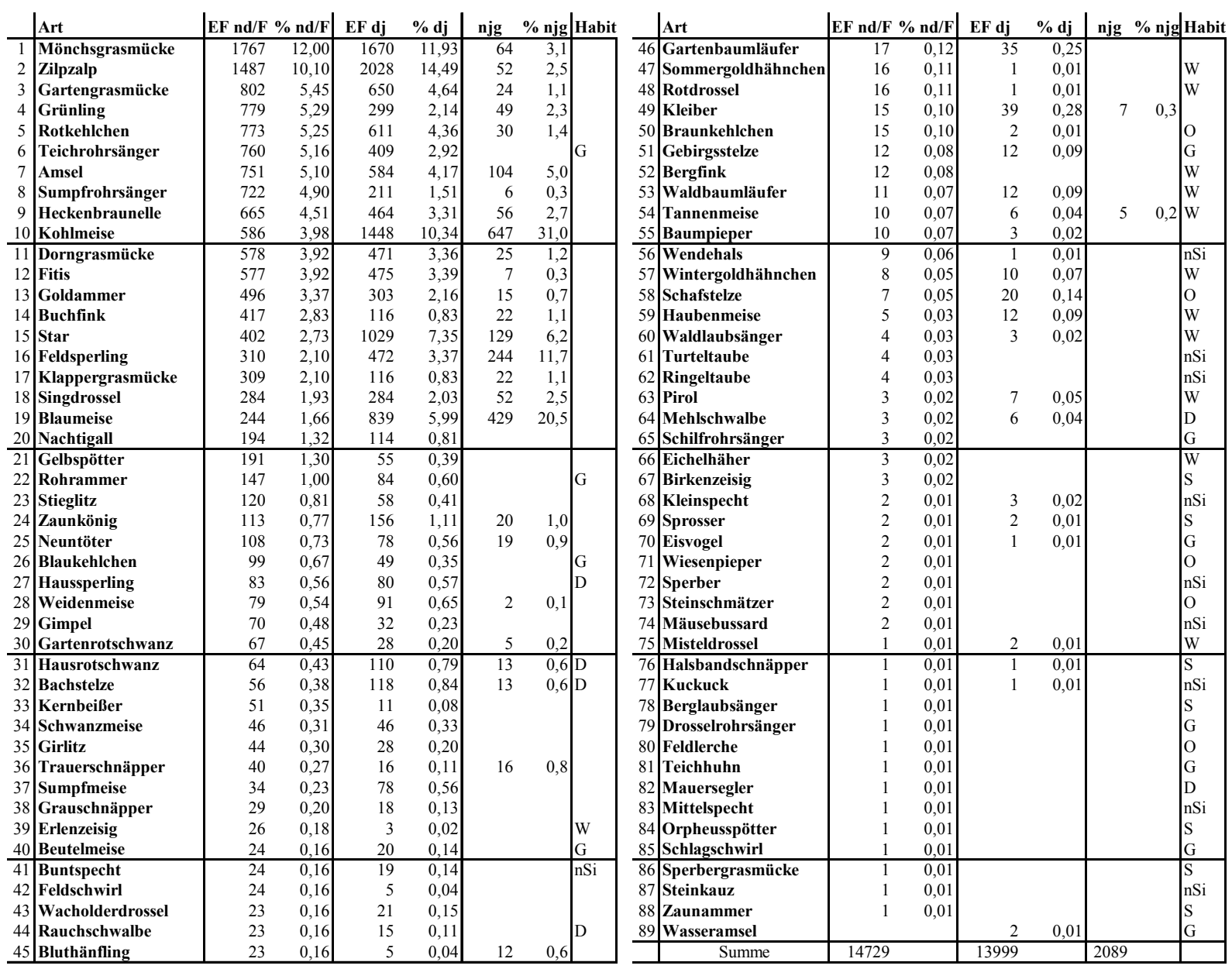


Tab. 46: Dominanz der Erstfänge adulter Vögel in geschlossenen Hecken bzw. in Hecken mit lückigem Gehölzbestand.

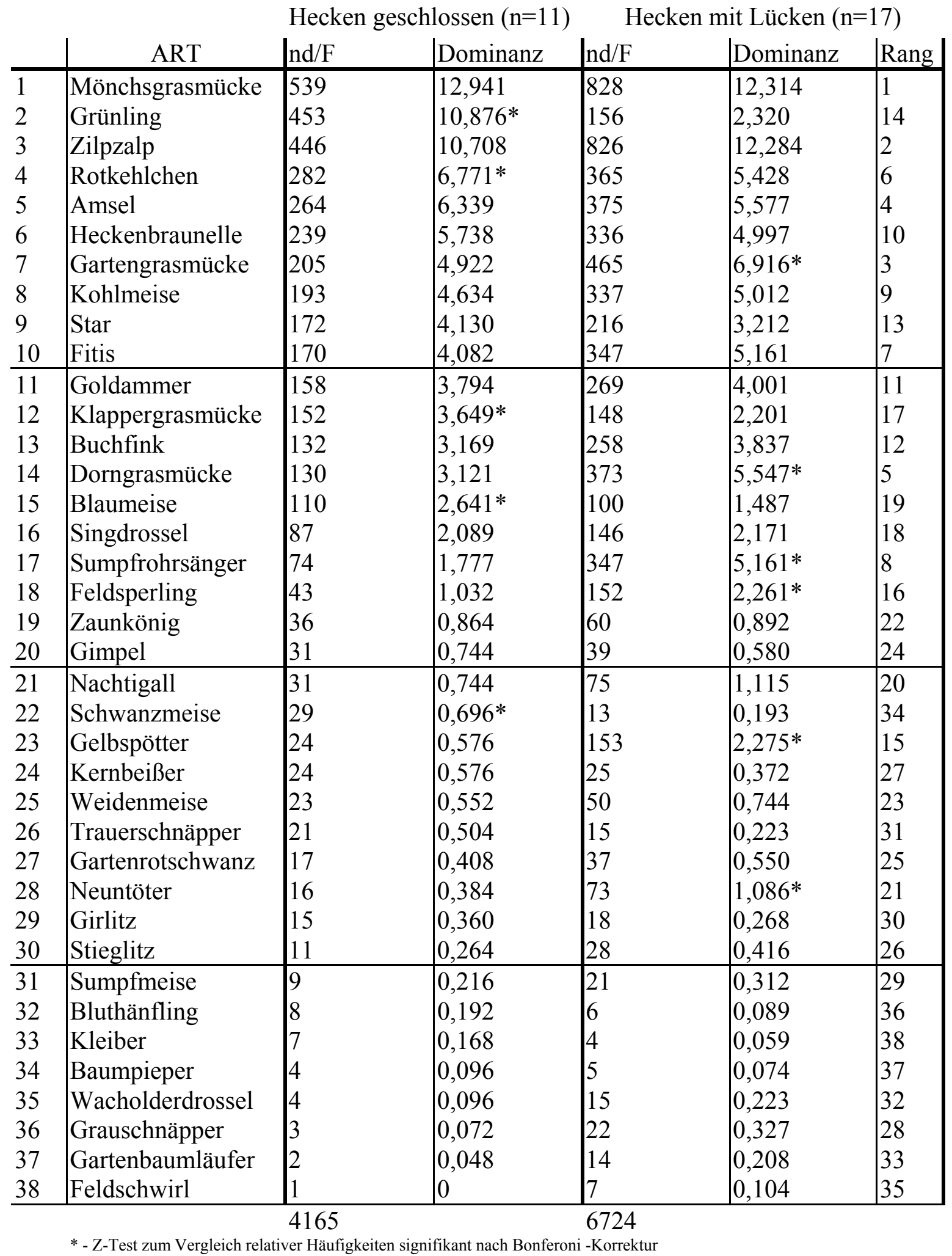


Tab. 47: Dominanz der Erstfänge adulter Vögel in Hecken über 6m durchschnittlicher Breite bzw. geringerer Breite.

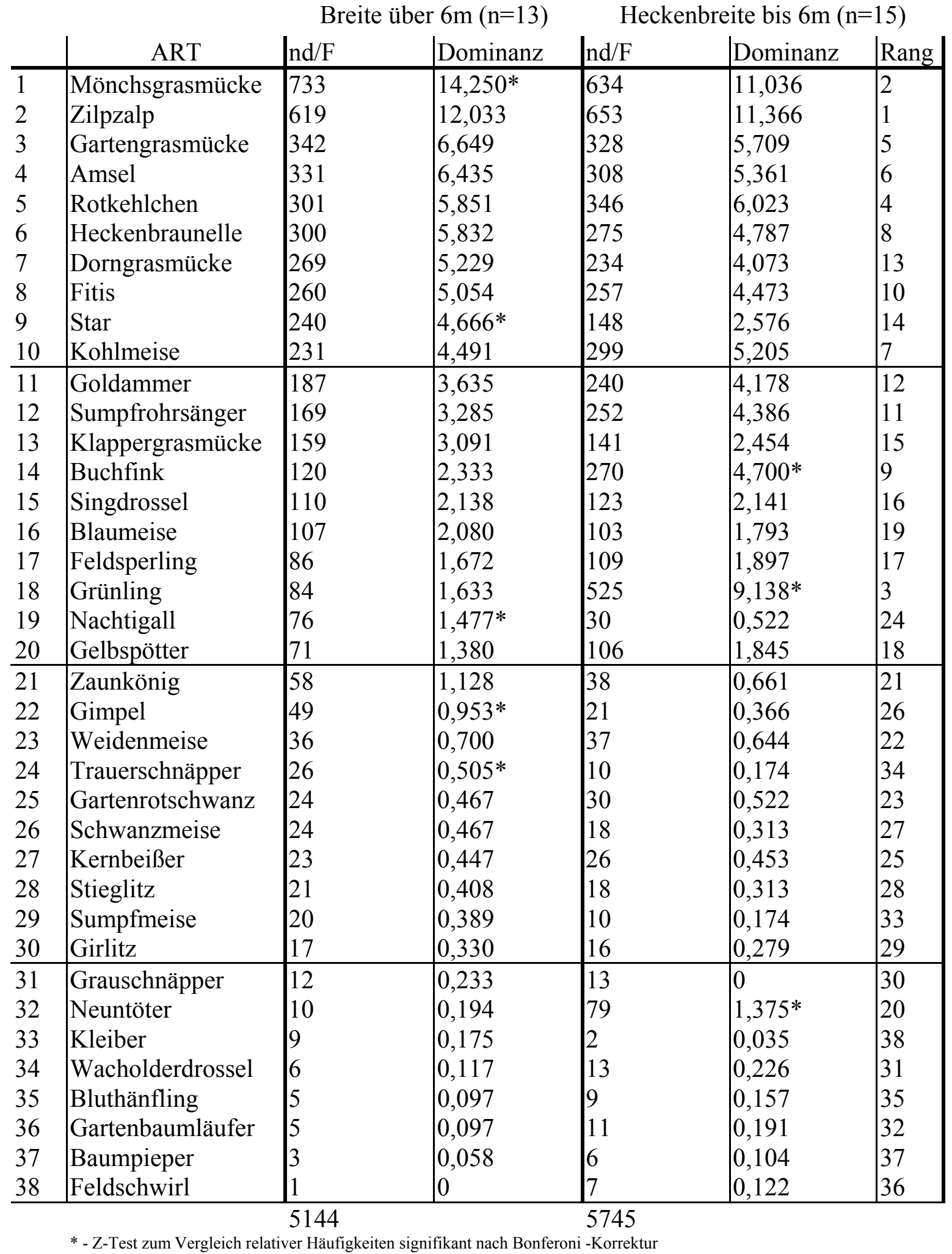


Tab. 48: Dominanz der Erstfänge adulter Vögel in Hecken mit maximal $8 \mathrm{~m}$ und mehr Höhe bzw geringerer Höhe.

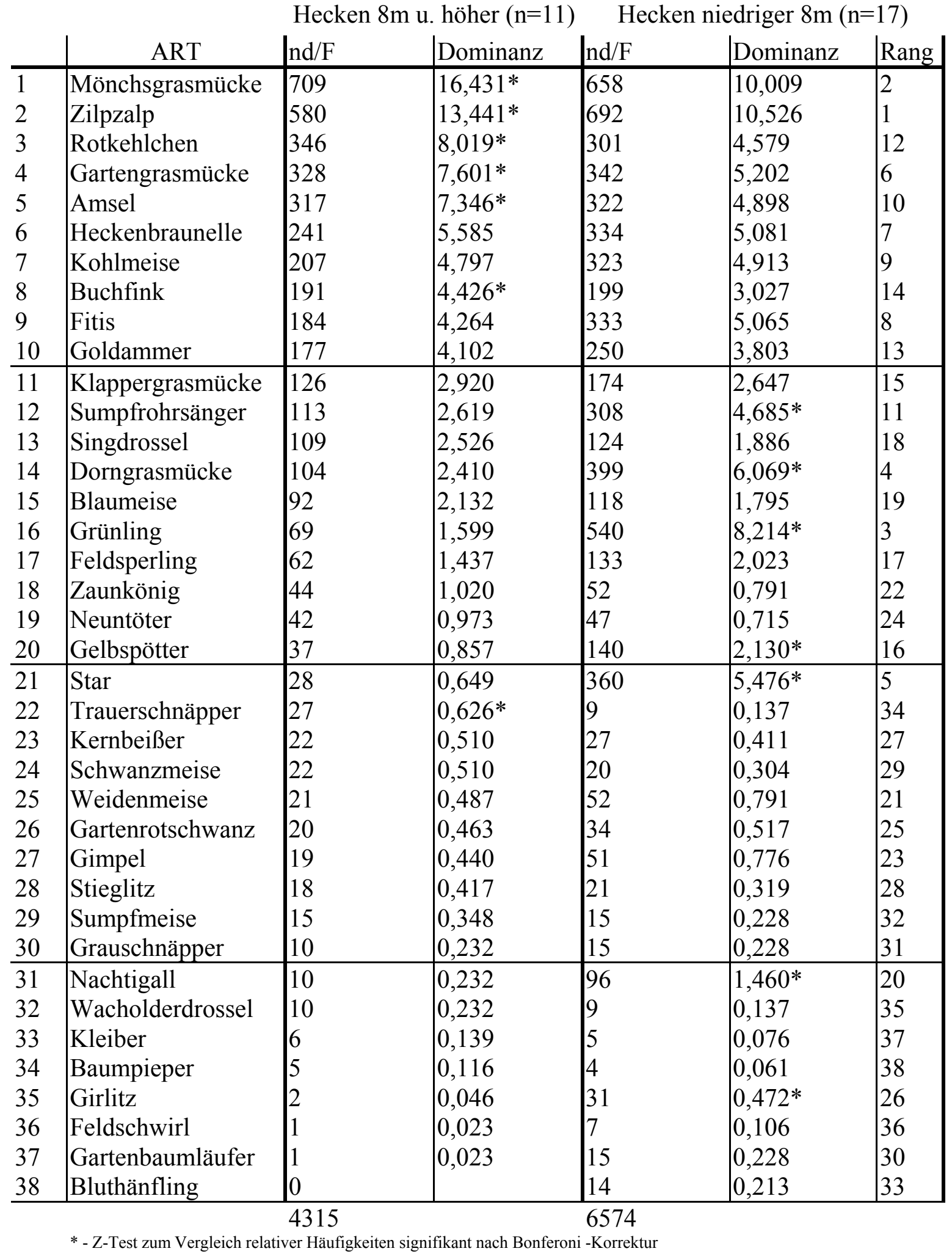




\section{Fangsummen und Bestandsveränderungen der Jungvögel}

Tab. 49: Fänge juveniler Vögel als Anzahl auf 100 Fangtage, mit Angabe der Veränderung zum Vorjahr; n=23 Hecken ununterbrochener Bearbeitung; $\square$ =positive Populationsentwicklung, $\square=$ negative Populationsentwicklung, Veränderungen unter $10 \%$ nicht gekennzeichnet.

\begin{tabular}{|c|c|c|c|c|c|c|c|c|}
\hline Art & 1994 & 1995 & 1996 & $\begin{array}{r}\% 1996 \\
\text { zu } 1995\end{array}$ & 1997 & $\begin{array}{r}\text { \% } 1997 \\
\text { zu } 1996\end{array}$ & 1998 & $\begin{array}{l}\text { \% } 1998 \\
\text { zu } 1997\end{array}$ \\
\hline Amsel & 37,4 & 52 & 48,6 & $-6,7$ & 34,5 & $-28,9$ & 39,3 & 13,9 \\
\hline Singdrossel & 17,9 & 21,4 & 30,5 & 42,1 & 15 & $-50,6$ & 25,7 & 71,1 \\
\hline Nachtigall & 0,56 & 5,61 & 10,7 & 90,7 & 13,7 & 28,2 & 22,3 & 62,7 \\
\hline Rotkehlchen & 29,6 & 48,5 & 46,9 & $-3,3$ & 48,7 & 3,8 & 65,5 & 34,6 \\
\hline KohImeise & 124 & 134 & 169 & 26,5 & 162 & -4 & 164 & 0,7 \\
\hline Blaumeise & 58,1 & 69,4 & 94,7 & 36,4 & 73 & $-22,9$ & 72,3 & $-0,1$ \\
\hline Weidenmeise & 5,03 & 6,63 & 8,64 & 30,3 & 10,2 & 17,8 & 6,31 & $-39,1$ \\
\hline Star & 16,2 & 20,4 & 24,3 & 19 & 358 & 1372 & 129 & $-63,9$ \\
\hline Mönchsgrasmücke & 73,2 & 96,9 & 83,1 & $-14,2$ & 106 & 27,8 & 185 & 73,7 \\
\hline Gartengrasmücke & 14,5 & 24,5 & 39,5 & 61,3 & 85,8 & 117 & 75,7 & $-11,8$ \\
\hline Dorngrasmücke & 2,79 & 42,4 & 32,1 & $-24,2$ & 48,2 & 50,2 & 51 & 5,7 \\
\hline Klappergrasmücke & 3,91 & 15,8 & 8,23 & -48 & 13,7 & 66,7 & 11,7 & $-15,1$ \\
\hline Fitis & 15,6 & 70,4 & 50,2 & $-28,7$ & 57,1 & 13,7 & 78,6 & 37,7 \\
\hline Zilpzalp & 46,9 & 149 & 154 & 3,2 & 173 & 12,2 & 221 & 28,3 \\
\hline Goldammer & 1,68 & 23,5 & 20,6 & $-12,3$ & 19 & $-7,5$ & 35,9 & 88,8 \\
\hline Sumpfrohrsänger & 2,24 & 11,7 & 9,47 & $-9,3$ & 16,8 & 77,5 & 10,7 & $-36,5$ \\
\hline Neuntöter & 0,56 & 9,18 & 2,88 & $-68,6$ & 6,64 & 131 & 3,4 & $-48,8$ \\
\hline Heckenbraunelle & 24,6 & 34,2 & 43,2 & 26,4 & 47,4 & 9,6 & 54,9 & 15,8 \\
\hline Zaunkönig & 14 & 14,3 & 11,1 & $-22,2$ & 12 & 7,6 & 9,22 & $-22,9$ \\
\hline Gelbspötter & 1,12 & 3,06 & 5,35 & 74,8 & 7,08 & 32,3 & 5,83 & $-17,7$ \\
\hline Buchfink & 2,79 & 9,18 & 11,1 & 21 & 7,08 & $-36,3$ & 10,2 & 43,9 \\
\hline Grünling & 34,1 & 43,4 & 29,6 & $-31,7$ & 23,5 & $-20,9$ & 4,85 & $-79,4$ \\
\hline
\end{tabular}




\section{Abundanzveränderungen bei Jungvögeln}

Tab. 50: $\quad$ Ergebnis der Konfigurations-Frequenz-Analyse (KFA) zu Abundanzveränderungen der Jungvögel; $\square$ = signifikant niedrigere, $\square$ = signifikant höhere Fangzahl.

\begin{tabular}{|c|c|c|c|c|c|c|c|}
\hline & ART & 1994 & 1995 & 1996 & 1997 & 1998 & gesamt \\
\hline 1 & Amsel & 78 & 118 & 77 & 51 & 77 & 401 \\
\hline 2 & Baumpieper & 0 & 0 & 2 & 0 & 1 & 3 \\
\hline 3 & Blaumeise & 83 & 104 & 139 & 117 & 140 & 583 \\
\hline 4 & Bluthänfling & 0 & 2 & 0 & 0 & 0 & 2 \\
\hline 5 & Buchfink & 21 & 14 & 29 & 24 & 17 & 105 \\
\hline 6 & Dorngrasmücke & 51 & 96 & 80 & 63 & 77 & 367 \\
\hline 7 & Feldschwirl & 1 & 1 & 0 & 1 & 0 & 3 \\
\hline 8 & Feldsperling & 18 & 83 & 26 & 19 & 13 & 159 \\
\hline 9 & Fitis & 58 & 133 & 119 & 63 & 87 & 460 \\
\hline 10 & Gartenbaumläufer & 12 & 3 & 2 & 2 & 0 & 19 \\
\hline 11 & Gartengrasmücke & 51 & 81 & 71 & 59 & 79 & 341 \\
\hline 12 & Gartenrotschwanz & 0 & 9 & 3 & 5 & 4 & 21 \\
\hline 13 & Gelbspötter & 7 & 8 & 14 & 10 & 12 & 51 \\
\hline 14 & Gimpel & 7 & 6 & 7 & 4 & 6 & 30 \\
\hline 15 & Girlitz & 1 & 1 & 8 & 2 & 5 & 17 \\
\hline 16 & Goldammer & 21 & 47 & 43 & 48 & 83 & 242 \\
\hline 17 & Grauschnäpper & 0 & 4 & 7 & 2 & 5 & 18 \\
\hline 18 & Grünling & 52 & 79 & 53 & 40 & 15 & 239 \\
\hline 19 & Heckenbraunelle & 66 & 72 & 67 & 82 & 102 & 389 \\
\hline 20 & Kernbeißer & 0 & 0 & 1 & 2 & 7 & 10 \\
\hline 21 & Klappergrasmücke & 26 & 30 & 22 & 15 & 21 & 114 \\
\hline 22 & Kleiber & 3 & 9 & 10 & 4 & 9 & 35 \\
\hline 23 & Kohlmeise & 208 & 189 & 327 & 274 & 251 & 1249 \\
\hline 24 & Mönchsgrasmücke & 233 & 221 & 152 & 171 & 254 & 1031 \\
\hline 25 & Nachtigall & 6 & 11 & 16 & 16 & 21 & 70 \\
\hline 26 & Neuntöter & 4 & 19 & 8 & 13 & 7 & 51 \\
\hline 27 & Rotkehlchen & 99 & 123 & 93 & 106 & 130 & 551 \\
\hline 28 & Schwanzmeise & 3 & 12 & 17 & 8 & 5 & 45 \\
\hline 29 & Singdrossel & 36 & 43 & 52 & 36 & 63 & 230 \\
\hline 30 & Star & 14 & 15 & 11 & 749 & 207 & 996 \\
\hline 31 & Stieglitz & 9 & 4 & 1 & 3 & 22 & 39 \\
\hline 32 & Sumpfmeise & 11 & 20 & 14 & 16 & 12 & 73 \\
\hline 33 & Sumpfrohrsänger & 16 & 26 & 25 & 30 & 28 & 125 \\
\hline 34 & Trauerschnäpper & 8 & 0 & 2 & 2 & 1 & 13 \\
\hline 35 & Wacholderdrossel & 3 & 1 & 4 & 2 & 7 & 17 \\
\hline 36 & Weidenmeise & 10 & 13 & 18 & 6 & 16 & 63 \\
\hline 37 & Zaunkönig & 22 & 37 & 27 & 24 & 22 & 132 \\
\hline 38 & Zilpzalp & 173 & 340 & 363 & 381 & 443 & 1700 \\
\hline & Summe ohne Nr. 18 u. 30 & 1345 & 1880 & 1846 & 1661 & 2027 & 8759 \\
\hline
\end{tabular}




\section{Tabellenverzeichnis}

Tab. 1: Die ehrenamtlichen Mitarbeiter und Orte der Untersuchungsgebiete, nach PLZ sortiert.

Tab. 2: Sichere und fragliche Brutpaare (BP) sowie der Anteil fraglicher BP (\%) der im Heckenprogramm über fünf Jahre erfassten Reviere in 33 Hecken mit $>10$ BP. BP si, $\mathrm{BP}$ fr $=$ sichere bzw. fragliche Brutpaare, $\% \mathrm{fr}=$ Anteil fraglicher Brutpaare, $*=$ Vögel die (methodisch bedingt) nicht durch Netzfang nachgewiesen werden konnten.

Tab. 3: Einteilung der Dekaden und numerische Zuordnung.

Tab. 4: Durchschnittliche Anzahl der jährlich durchgeführten Fangtage bei allen 23 Bearbeitern.

Tab. 5: Die Programmvögel des Heckenprogramms der deutschen Vogelwarten, in alphabetischer Reihenfolge.

Tab. 6: Übersicht der Beringungszahlen von adulten und juvenilen Erstfängen und Nestlingen der Programmvögel sowie Dominanz [\%] jeder Art für das betreffende Alter.

Tab. 7: Dominanz der Erstfänge adulter Vögel in Hecken über $(n=9)$ bzw. bis 20 Jahre Alter $(\mathrm{n}=19)$; Rang bezieht sich auf die Dominanz der Arten in Hecken bis 20 Jahre. ...... 35

Tab. 8: Unterschiede in der Bevorzugung von 4 Heckenparametern durch ausgewählte Vogelarten, je Z-Test mit $\mathrm{N}=28$ und $\mathrm{p}<0,01$ (vgl. Ergebnisse in Tab. 7, Tab. 46, Tab. 47 und Tab. 48).

Tab. 9: Strukturwahl von Singvögeln in Hecken. Eingefärbte Flächen zeigen signifikante Bevorzugung einer Strukturausprägung der Hecken durch die betreffende Vogelart an (Z-Test mit Korrektur nach Bonferoni). Dunkle Flächen zeigen ausschließliche Bevorzugung von waldartigen, hell die von halboffenen Strukturen. 38

Tab. 10: Anzahl der durchschnittlichen Fangtage zur Beschreibung der Bestandsdynamik über $\mathrm{N}=21$ Hecken.

Tab. 11: Fänge adulter Vögel als Anzahl auf 100 Fangtage, mit Angabe der Veränderung zum Vorjahr; $n=21$ Hecken ununterbrochener Bearbeitung; $\square$ =positive

Populationsentwicklung, $\square$ =negative Populationsentwicklung, Veränderungen unter $10 \%$ nicht gekennzeichnet.

Tab. 12: Ergebnis der Konfigurations-Frequenz-Analyse (KFA) zu Abundanzveränderungen der Altvögel;

= signifikant niedrigere, $\square$ = signifikant höhere Fangzahl.

Tab. 13: Anzahl der Fangtage je Dekade zur Beschreibung der Fangsummen über N=25

Hecken, mit Angabe der Abweichung je Dekade vom Mittelwert über alle Dekaden.46

Tab. 14: Phänologiedaten der häufigsten Heckenvögel $(n=20)$ aus 25 Hecken und

Bestimmung der Dekaden mit geringem Einfluss der Fangergebnisse durch Migration (Zug minimal). Dekaden entsprechend der Festlegung in Tab. 3, weitere Abk. im Text.

Tab. 15: Indizierung des Bruterfolgs über das Verhältnis gefangener Jungvögel zu den Altvögeln (I), als Index aus dj/nd Fänglingen (unter Einschluss aller Erstfänge sowie der in Vorjahren beringten Vögel) und die prozentuale Veränderung dieses Index zum Vorjahr (\% VJ). MW bezeichnet den Mittelwert des Index und unter $\mathrm{N}$ ist die Anzahl erfasster Individuen über alle Untersuchungsjahre angegeben. 
Tab. 16: Einflüsse von Faktoren, die den Bruterfolg beeinträchtigen können.

Tab. 17: Wiederfänge (W) adulter Programmvögel und deren Anteil am Gesamtfang der Art (\%), $\mathrm{N}=$ Gesamtfang der Altvögel in 23 Hecken.

Tab. 18: Wiederfänge (W) von adulten Programmarten (mit W > 20) und deren Anteil an Erstfängen (\%) zwischen 1994 bis 1998 sowie die Differenz zur Summe der Wiederfänge vom Vorjahr (D). W (ges) =Anzahl aller Wiederfänge, \% (MW) $=$ Mittelwert der jährlichen Anteile und $\mathrm{N}=$ Gesamtfang nd/F der Art.

Tab. 19: Vergleich der Dominanz der 18 häufigsten Programmvögel.

Tab. 20: Anzahlen der Reviere von Brutvögeln in Hecken, die von 1998 bis 2000 kartiert wurden. Brutvögel $(\mathrm{N}=41)$ und Hecken $(\mathrm{N}=30)$ sind nach Häufigkeit bzw. Artenzahl sortiert. Unter 'Ort' sind die Untersuchungsgebiete angegeben; mit Ge - Geismar, Ap Appenrode-Gleichen, Vo - Volkerode, Jü - Jühnde und Le - Lemshausen. Unter 'Hecke (Länge) Arten' folgt eine Heckennummer und darunter in Klammern die Länge der betreffenden Hecke sowie die Artenzahl. Alle Angaben einschließlich der Summen der Brutpaare (BP) und der Brutpaare je Art sind Anzahlen ohne Flächenbezug. Mit* gekennzeichnete Arten profitierten von künstlichen Nisthöhlen in einigen (*) Hecken. 98

Fortsetzung von Tab. 20: Anzahlen der Reviere von Brutvögeln in Hecken. 99

Tab. 21: Entwicklung der Revierzahlen aller Brutvögel über alle 30 Hecken. 100

Tab. 22: Anzahl besiedelter Hecken (von N=30), unterschieden nach alljährlicher (über drei Jahre) und zeitweiliger Nutzung als Brutrevier. Unter C und D sind Anteile zeitweise besiedelter Hecken bzw. Reviere angegeben. 101

Tab. 23: Arten, die in den untersuchten 30 Hecken (zusätzlich) auftreten, in denen die jeweils angegebene Artenzahl vorhanden ist, eingeklammert ist die Anzahl der Hecken mit der entsprechenden Artenzahl.

Tab. 24: Abundanz [BP/km] und Dominanz [\%] der Arten in 30 Hecken zwischen 1998-2000. Gekennzeichnete Arten brüteten *teilweise oder **ausschließlich in Nistkästen. .. 104

Tab. 25: Multiple Lineare Regression für Brutpaare je Kilometer Hecke mit korr. $\mathrm{R}^{2}=0,69$, F $(5,24)=13,923, \mathrm{p}<0,001$, SF der Schätzung: 23,078, $\mathrm{N}=30$..

Tab. 26: Ergebnis der Hauptkomponentenanalyse: Faktorladungen (Varimax standard Rotation) und Extraktion der Hauptkomponenten mit Ladungen >0,6 markiert. .... 106

Tab. 27: Chi ${ }^{2}$-Test zur Feststellung der Anzahl extrahierbarer Roots................................ 106

Tab. 28: Ergebnis der Kanonischen Korrelation: Faktorenstruktur und Extraktion markierter Ladungen. 107

Tab. 29: Anzahl und Anteil der erfolgreichen und der durch Prädation oder andere Ursachen verlorenen Nester. 108

Tab. 30: Bruterfolg häufiger Brutvögel in Hecken, 1998-2000. 108

Tab. 31: Ermittlung der durchschnittlichen Nestdauer (ND) aus beobachteter Brutdauer und der mittleren Nestlingszeit nach Literaturangaben. 109

Tab. 32: Die Überlebenswahrscheinlichkeit $\left(\operatorname{SURV}_{(\mathrm{t})}\right)$ von Singvogelnestern in Hecken berechnet nach der Mayfield-Methode. Zu den verwendeten Abkürzungen siehe Text und Methodik.

Tab. 33: Ausfliegeerfolg berechnet aus der mittleren Gelegegröße (ø Eizahl, Tab. 30) aller Vollgelege und der Gesamt-Überlebenswahrscheinlichkeit von Nestern $\left(\mathrm{SURV}_{\mathrm{t}}\right.$ aus 
Tab. 32). Zum Vergleich die aus der Anzahl der ausgeflogenen Jungvögel je Nest ermittelten Werte aus Tab. 30 .

Tab. 34: Mortalitätsraten für Alt- $\left(\mathrm{M}_{\mathrm{ad}}\right)$ und Jungvögel $\left(\mathrm{M}_{\mathrm{juv}}\right)$ nach Literaturangaben und Anzahl der Brutversuche B und $B_{S}$ nach Angaben von SIRIWARDENA et al. (1998), Schätzwerte kursiv.

Tab. 35: Relative Populationsgröße [BP] heckenbrütender Singvögel in einem Folgejahr, basierend auf den erhobenen Ausfliegeerfolgen der Arten. Zu den unterschiedlichen Berechnungsvarianten siehe Text.

Tab. 36: Brutverluste durch Prädation und sonstige Ursachen an acht Singvogelarten in Hecken.

Tab. 37: Vergleich der prozentualen Verteilung gefundener und ausgeraubter Nester in der Brutzeit.

Tab. 38: Anteil der Nestverluste bei Dorngrasmücke ( $\mathrm{N}=17$ Verluste gesamt) und Goldammer $(\mathrm{N}=24)$ durch Störungen und das Ausmähen von Krautvegetation an Hecken........ 116

Tab. 39: Anzahl der ausgeraubten Kunstnester mit Kamera durch Elster und Eichelhäher. 117

Tab. 40: Anzahl und Anteil leerer Kunstnester nach drei bzw. sieben Tagen (d)

Expositionszeit. Mit* gekennzeichnete Werte weichen nach Z-Test signifikant von den Anteilen leerer Nester in den anderen Zeiträumen ab (* entspricht jeweils $p<0,01$ ).

Tab. 41: Ergebnis der schrittweise vorwärts durchgeführten binären Logistischen Regression, mit $\mathrm{N}=496, \mathrm{FG}=4, \mathrm{p}=0,001$.

Tab. 42: Ergebnis der schrittweise vorwärts durchgeführten binären Logistischen Regression, mit $\mathrm{N}=41, \mathrm{FG}=3, \mathrm{p}=0,007$. 120

Tab. 43: Besiedlungsdichte einiger Habitate durch Vögel, nach FLADE 1994. 124

Tab. 44: Gesamtzahl der Brutpaare (BP) und Dominanz (D) der Programmvögel über fünf Jahre in 33 Hecken.

Tab. 45: Übersicht der Beringungszahlen von adulten und juvenilen Erstfängen und Nestlingen sowie Dominanz [\%] jeder Art für das betreffende Alter, Abk. im Text, unter 'Habit' sind Arten gekennzeichnet, die aufgrund ihrer Habitatwahl oder Verbreitung nur unter besonderen Umständen in Hecken siedeln, siehe dazu 'Auswahl der Programmvögel'.

Tab. 46: Dominanz der Erstfänge adulter Vögel in geschlossenen Hecken bzw. in Hecken mit lückigem Gehölzbestand.

Tab. 47: Dominanz der Erstfänge adulter Vögel in Hecken über $6 \mathrm{~m}$ durchschnittlicher Breite bzw. geringerer Breite.

Tab. 48: Dominanz der Erstfänge adulter Vögel in Hecken mit maximal 8m und mehr Höhe bzw geringerer Höhe 168

Tab. 49: Fänge juveniler Vögel als Anzahl auf 100 Fangtage, mit Angabe der Veränderung zum Vorjahr; $n=23$ Hecken ununterbrochener Bearbeitung; $\square$ =positive Populationsentwicklung, $\square$ =negative Populationsentwicklung, Veränderungen unter $10 \%$ nicht gekennzeichnet.

Tab. 50: Ergebnis der Konfigurations-Frequenz-Analyse (KFA) zu Abundanzveränderungen der Jungvögel; $\square$ = signifikant niedrigere, $\square$ = signifikant höhere Fangzahl....... 170 


\section{Abbildungsverzeichnis}

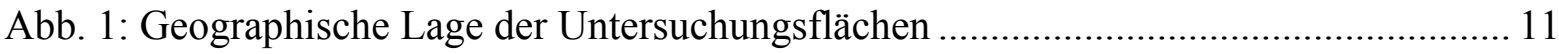

Abb. 2: Mediane der Brutpaardichte und Diversität von Vögeln in $\mathrm{N}=27$ Hecken.............. 20

Abb. 3: Varianz der von allen Bearbeitern $(n=23)$ durchgeführten Anzahl von Fangtagen über fünf Jahre Untersuchungszeitraum. Die Anzahl der jährlich bearbeiteten

Untersuchungsgebiete ist in Tab. 4 angegeben.

Abb. 4: Jahresberingungssummen adulter (nd), juveniler (dj) und nestjunger (njg)

Programmvögel in 33 Hecken.

Abb. 5: Darstellung durchschnittlicher jährlicher Fangsummen aller Programmvogelarten in Hecken nach Strukturmerkmalen. Angegeben sind die Mittelwerte mit

Standardabweichung und Standardfehler der gemittelten Fangsummen pro Hecke und Jahr für Alter*, Breite, Höhe und Lückigkeit.

Abb. 6: Darstellung unterschiedlicher Dominanzen von vier Grasmücken-Arten in Hecken verschiedenen Alters. $\mathrm{Mg}=$ Mönchsgrasmücke, $\mathrm{Gg}=$ Gartengrasmücke, $\mathrm{Kg}=$ Klappergrasmücke, $\mathrm{Dg}=$ Dorngrasmücke; für alle Arten ist der Unterschied zwischen jungen und alten Hecken signifikant, Z-Test, $\mathrm{N}=28, \mathrm{p}<0,0001$.

Abb. 7: Dekadensummen beringter Altvögel (nd/F) und Jungvögel (dj) aus 25 Hecken in fünf Jahren Heckenprogramm. Für n=18 Arten, Daten dem Fangaufwand in den Dekaden entsprechend angepasst.

Abb. 8: Dekadensummen beringter Mönchs- und Gartengrasmücken aus 25 Hecken in fünf Jahren Heckenprogramm. Daten dem Fangaufwand entsprechend angepasst.

Abb. 9: Dekadensummen beringter Dorn- und Klappergrasmücken aus 25 Hecken in fünf Jahren Heckenprogramm. Daten dem Fangaufwand entsprechend angepasst.

Abb. 10: Dekadensummen ziehender Singvogelarten aus 25 Hecken in fünf Jahren

Heckenprogramm.

Abb. 11: Dekadensummen beringter Standvögel und Teilzieher aus 25 Hecken in fünf Jahren Heckenprogramm.

Abb. 12: Der „relative Bruterfolg“ aus dem Verhältnis der Jung- zu den Altvögeln als Summierung über 16 Arten mit N=11.871 Fänglingen aus 25 Hecken und die jeweilige prozentuale Veränderung zum Vorjahr (Arten wie in Tab. 15, ohne Feldsperling und Blaumeise).

Abb. 13: Der „relative Bruterfolg“ aus dem Verhältnis der Jung- zu den Altvögeln für Mönchs- und Dorngrasmücke, Amsel und Goldammer aus 25 Hecken und die jeweilige prozentuale Veränderung zum Vorjahr, für N der Arten siehe Tab. 15. .... 60

Abb. 14: Fangzeitpunkte von Erstfängen des Zilpzalp, die später als Wiederfänge registriert werden konnten (23 Hecken zwischen 1994-1998, n=184).

Abb. 15: Beobachteter Anteil der Wiederfänge des Zilpzalps im Dekadenvergleich mit dem Anteil adulter Erstfänge (Erwartungswert) über KFA. Die Werte sind zur besseren Vergleichbarkeit durch die Grundgesamtheit geteilt.

Abb. 16: Zeiträume zwischen Erstfang und Wiederfang beim Zilpzalp zwischen 1994-1998 in 23 Hecken $(n=184)$.

Abb. 17: Korrelation von Brutpaaren und Erstfängen der 30 häufigsten Programmvögel.... 69 
Abb. 18: Wiederfänge [W] und durch Revierkartierung ermittelte Brutpaare [BP] sowie der Anteil von Wiederfängen an den Brutpaaren [\% W an BP] ausgewählter Arten....... 72

Abb. 19: Übersichtskarte der Untersuchungsgebiete um Göttingen. ................................... 82

Abb. 20: Heckenlandschaft bei Volkerode am 21. April 2000: von Schlehen dominierte Hecken sind in intensiv genutzte Kulturlandschaft eingefügt, im Hintergrund liegt Göttingen. 83

Abb. 21: Luftbild Volkerode im Maßstab 1:10.000 vom 17. Mai 1993 84

Abb. 22: Hecke 45 bei Jühnde, mit einer mehrjährigen Brachefläche im Vordergrund 85

Abb. 23: Zur Anschauung freigestellte Kunstnester vor der Plazierung in der Hecke .94

Abb. 24: Beziehung zwischen Brutpaaren pro Kilometer und Heckenbreite (A) und Wegedichte (B). 105

Abb. 25: Beziehung Brutpaare pro Kilometer bzw. Artenzahl mit Länge der Hecken........ 105

Abb. 26: Nesthöhe bei der Goldammer in Abhängigkeit von der Jahreszeit (U-Test, $p<0,02$, $\mathrm{N}=41$, zweiseitig.

Abb. 27: Zeitlicher Verlauf des Anteils durch Prädation verlorener Nester offen brütender Singvögel. Die Nester sind ihres Fundtages entsprechend datiert.....

Abb. 28: Freigemähtes Nest der Dorngrasmücke am 27. Mai 1999 (links) und zwei Tage später ausgeraubt (rechts).

Abb. 29: Eichelhäher und Elster als Nesträuber an Kunstnestern

Abb. 30: Zusammenhang zwischen Heckenbreite und Ausraubrate von Kunstnestern nach sieben Tagen Expositionszeit.

Abb. 31: Beziehung der Ausraubwahrscheinlichkeit eines Kunstnestes in Abhängigkeit des Schicksals der Nachbarnester. 


\section{Verzeichnis von Worterklärungen und Abkürzungen}

\begin{tabular}{|c|c|}
\hline Abundanz & $\begin{array}{l}\text { Anzahl von Vögeln (allg. Organismen) bezogen auf } \\
\text { eine Bezugseinheit }\end{array}$ \\
\hline $\begin{array}{l}\text { British Trust for } \text { Ornithology } \\
\text { (BTO) }\end{array}$ & $\begin{array}{l}\text { privat organisierte Naturschutzvereinigung (NGO) in } \\
\text { UK, die auch Aufgaben des Monitorings an Vogel- } \\
\text { populationen wahrnimmt }\end{array}$ \\
\hline Constant Effort Site (CES) & $\begin{array}{l}\text { Synonym für Fangprogramm des BTO in UK, bei dem } \\
\text { an den Fangplätzen (sites) nach streng standardisierter } \\
\text { Vorgabe beringt wird. Von zentraler Bedeutung ist, } \\
\text { dass der Fangbetrieb mit konstantem Aufwand } \\
\text { (constant effort) durchgeführt wird }\end{array}$ \\
\hline Dekade & Zeitabschnitt von 10 Kalendertagen \\
\hline $\mathrm{dj}$ & $\begin{array}{l}\text { diesjährig, juvenil, Jungvogel; exakte Bezeichnung zur } \\
\text { Protokollierung des Alters in einem Kalenderjahr }\end{array}$ \\
\hline Dominanz & $\begin{array}{l}\text { relative Menge einer Art zu den insgesamt erfassten } \\
\text { Individuen aller Arten. Eudominant sind Arten mit } \\
\text { mehr als } 10 \% \text { der Gesamtindividuenmenge, es folgen } \\
\text { dominante }(5-10 \%) \text {, subdominante }(2-5 \%) \text {, rezedente } \\
(1-2 \%) \text { und subrezedente }(<1 \%) \text { Arten; nach } \\
\text { MÜHLENBERG (1993) und SCHAEFER }(1992)\end{array}$ \\
\hline Erstfang & $\begin{array}{l}\text { Vögel die zum ersten mal zur Beringung gefangen } \\
\text { werden }\end{array}$ \\
\hline eudominant & siehe Dominanz \\
\hline eurytop & $\begin{array}{l}\text { Vögel oder andere Organismen die in vielen verschie- } \\
\text { denen Lebensräumen vorkommen können }\end{array}$ \\
\hline Fängling & $\begin{array}{l}\text { Bezeichnung für gefangenen Vogel unbestimmten } \\
\text { Alters, Zusammenfassung von gefangenen, nicht näher } \\
\text { spezifizierten Vögeln }\end{array}$ \\
\hline Floater & $\begin{array}{l}\text { Nichtbrütende Individuen, die während der Brutperiode } \\
\text { revierlos und unverpaart sind }\end{array}$ \\
\hline halboffene Landschaft & $\begin{array}{l}\text { im Gegensatz zur Offenlandschaft (waldfrei- und quasi } \\
\text { ohne jegliche Gehölzstrukturen) kommen in der } \\
\text { halboffenen Landschaft Gehölze vor, die in ihrer } \\
\text { Ausprägung von Einzelbäumen bis zu Waldfragmenten } \\
\text { reichen können }\end{array}$ \\
\hline IMS & Integriertes Monitoring von Singvogelpopulationen \\
\hline integriertes Monitoring & $\begin{array}{l}\text { erweitertes Monitoring; im IMS werden über die } \\
\text { Bestandszählung an Vogelpopulationen (durch } \\
\text { Netzfang und Revierkartierung) hinaus auch der } \\
\text { relative Bruterfolg und Mortalitäts- und Rückkehrraten } \\
\text { von Singvogelpopulationen erfasst }\end{array}$ \\
\hline Heimzug & $\begin{array}{l}\text { (auch unpräzise Rückzug) Frühjahrszug aus den } \\
\text { Überwinterungsgebieten in die (hier: europäisch- } \\
\text { palearktischen) Brutgebiete }\end{array}$ \\
\hline Kalenderjahr & das Jahr vom 1. Januar bis zum 31. Dezember \\
\hline Kontrollfang & $\begin{array}{l}\text { Vogel der bereits einen Ring trägt und bereits vor dem } \\
\text { laufenden Kalenderjahr beringt worden ist }\end{array}$ \\
\hline Median & teilt eine Datenmenge in der Hälfte ihrer Anzahl \\
\hline
\end{tabular}




\begin{tabular}{|c|c|}
\hline nd & $\begin{array}{l}\text { nicht diesjährig, adult, Altvogel; exakte Bezeichnung } \\
\text { zur Protokollierung des Alters in einem Kalenderjahr }\end{array}$ \\
\hline Nichtsingvögel & $\begin{array}{l}\text { lat.: Nonpasseriformes, umfasst eine willkürlich (nicht } \\
\text { phylogenetisch begründet) zusammengefasste Gruppe } \\
\text { aller Vogelarten, die nicht den Singvögeln zugerechnet } \\
\text { werden (für einen Überblick der in Deutschland } \\
\text { nachgewiesenen Nichtsingvogelarten s. BEZZEL 1985) }\end{array}$ \\
\hline Nisthilfe & $\begin{array}{l}\text { erhöht das Angebot von Brutorten für Vögel, es } \\
\text { existiert ein vielfältiges Angebot für eine Vielzahl von } \\
\text { Arten, hier in der Regel Nistkästen für Höhlenbrüter }\end{array}$ \\
\hline njg & nestjung, Nestling \\
\hline Ökoton & $\begin{array}{l}\text { Grenzbereich zwischen verschiedenen Landschaften } \\
\text { (SCHAEFER 1992), Begriff wird in dieser Arbeit auf } \\
\text { Übergänge zwischen Landschaftsteilen (Wald- } \\
\text { Wiesenlandschaft) oder Landschaftselemente (Hecke- } \\
\text { Acker) bezogen benutzt }\end{array}$ \\
\hline Papierrevier & $\begin{array}{l}\text { aus mehreren Feldbeobachtungen einer Brutsaison 'auf } \\
\text { dem Papier' abgegrenztes Brutrevier }\end{array}$ \\
\hline Phänologie & $\begin{array}{l}\text { Lehre von den Erscheinungen des jahreszeitlichen } \\
\text { Ablaufs im Tier- und Pflanzenreich, z.B. Ankunfts- } \\
\text { zeiten von Zugvögeln }\end{array}$ \\
\hline Programmvögel & $\begin{array}{l}\text { spezifisch in dieser Arbeit benutzter Begriff; umfasst } \\
38 \text { Vogelarten, die durch Ausschluss von heckenun- } \\
\text { typischen Arten (z.B. Wasservögel) festgelegt wurden }\end{array}$ \\
\hline Recruitment & $\begin{array}{l}\text { Bezeichnet im hier benutzten Zusammenhang den } \\
\text { Zuwachs einer Population von einer Generation in die } \\
\text { folgende aufgrund von überlebenden Nachkommen } \\
\text { (ohne Zuwanderung) }\end{array}$ \\
\hline Standvogel & $\begin{array}{l}\text { Vogelarten, die nicht periodisch Zugbewegungen } \\
\text { zeigen; das schließt nicht aus, dass Teile der Population } \\
\text { unter bestimmten Bedingungen gerichtete Zugbe- } \\
\text { wegungen zeigen }\end{array}$ \\
\hline Stetigkeit & $\begin{array}{l}\text { Präsenz oder Vorkommen von bestimmten Arten; oft in } \\
\text { vier oder fünf Klassen von selten, wenig verbreitet, } \\
\text { verbreitet, häufig bis sehr häufig eingeteilt }\end{array}$ \\
\hline subrezedent & siehe Dominanz \\
\hline Teilzieher & $\begin{array}{l}\text { Vogelarten, bei denen nur ein Teil der Population } \\
\text { Zugverhalten zeigt }\end{array}$ \\
\hline Wegzug & $\begin{array}{l}\text { Herbstzug aus den Brutgebieten in die (meist südlich } \\
\text { gelegenen) Überwinterungsgebiete (Südeuropas und } \\
\text { Afrikas; Ausnahmen!) }\end{array}$ \\
\hline Wiederfang & $\begin{array}{l}\text { Vogel der bereits beringt ist und innerhalb des laufen- } \\
\text { den Kalenderjahres nochmals gefangen wird }\end{array}$ \\
\hline Zugvogel & $\begin{array}{l}\text { Vogelarten, die periodisch Wanderungen durchführen; } \\
\text { aus Mitteleuropa ziehen z.B. viele Zugvogelarten zur } \\
\text { Überwinterung in nahrungsreichere Gebiete und kehren } \\
\text { zur Brut wieder zurück }\end{array}$ \\
\hline
\end{tabular}

